\title{
Regulation of the Chromosomal Passenger Complex in Mitosis
}

\author{
Budhaditya Banerjee \\ Kolkata, India
}

B.S. Physiology, University of Calcutta - 2004

M.S. Biomedical Genetics, Vellore Inst. of Technology - 2006

\begin{abstract}
A Dissertation presented to the Graduate Faculty of the University of Virginia in Candidacy for the

Degree of Doctor of Philosophy
\end{abstract}

Department of Cell Biology

University of Virginia

November 2013 


\section{ABSTRACT}

The replicated genetic material is equally divided into two daughter cells during mitosis. Aurora B kinase plays a crucial role in achieving error free segregation of chromosomes. It generates the spindle checkpoint signal that ensures each kinetochore has made mature microtubule attachments and triggers pathways that correct erroneous kinetochore attachments. There is evidence that Aurora B kinase activity is regulated directly and also by controlling its localization at centromeres. At the beginning of this thesis work, it was not clear which was the dominant regulatory mechanism. Also, how CPC localizes to centromeres was unclear. This thesis is devoted to understanding the signaling pathways that mediate proper localization of Aurora B and regulation of its kinase activity. In Chapter 1 I will introduce the chromosome passenger complex (CPC) of which Aurora B is the catalytic subunit, its substrates and its overarching role in various mitotic processes. In Chapter 2 I will discuss how three different signaling pathways converge at the inner centromeres to localize Aurora B. In Chapter 3 I will highlight key cofactors that regulate Aurora B activity and elaborate its significance in context of the cell cycle. Finally, Chapter 4 will describe the complexity of Aurora B regulation. I will highlight its role as a control center for integrating signals from a multitude of sources to ensure error free chromosome segregation. I will also discuss some observed but yet unexplored events in mitosis. 


\section{ACKNOWLEDGMENTS}

First, I would like to thank my advisor Todd Stukenberg for his exceptional mentoring. He has guided me throughout my graduate career and contributed a lot in

making these years a great experience, both inside and outside the lab. I sincerely appreciate all his efforts in enhancing my skills and making me an independent scientist.

I would also like to thank Dan Burke and Dan Foltz for their valuable inputs throughout the duration of my thesis work. Their comments were critical in shaping the projects I worked on. Presenting in lab meetings would not have been the same learning experience without them.

I would cherish these years the most because I had the best colleagues at workplace one can ask for. I am very thankful for all the technical guidance and engaging discussions I had with past and present lab members - Weijie Lan, Sara Rosasco-Nitcher, John Tooley, Eliza Zylkiewicz, Ewa Niedzialkowska, Limin Liu, Cortney Kestner, Arkadi Manukyan, Prasad Trivedi, Pawel Janczyk and Dan Matson. My affiliation with both Biochemistry and Cell Biology departments gave me the opportunity to interact with two great communities. I would like to thank all my friends and faculty for making my graduate school experience so rewarding. I would like to specially thank Mary Hall for her support and help during these years.

Finally, last but by no means the least, I would like to thank my family - my dear wife Mandovi, for her unconditional love and support. She moved to Charlottesville from Pittsburgh so that we could be together. She is my rock. I would not be here without her. She gave me the reassurance I needed at moments when I had doubts. And my parents, 
for always encouraging me to follow my dreams and standing by my decisions. No 'thank you' is enough for the numerous sacrifices my mom and dad made to provide me with the best education. They always believed in me. This thesis is dedicated to them. I would also like to thank my in-laws for their love and support. 
TABLE OF CONTENTS

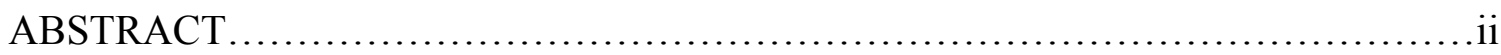

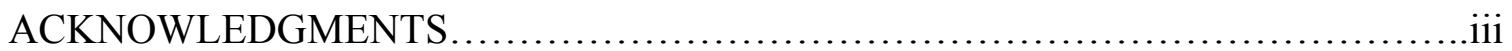

TABLE OF CONTENTS........................................................

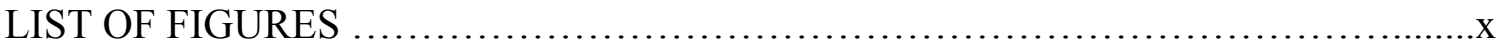

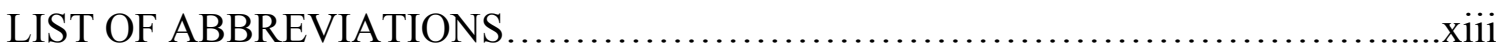

CHAPTER I: GENERAL INTRODUCTION

Mitosis and Chromosomal Passenger Complex.................................2

Mitotic Stages.................................................2

Aurora $B$ and its fellow passengers....................................4

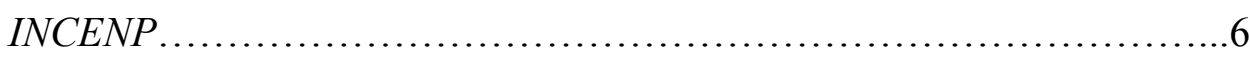

Survivin ...................................................6

Dasra / Borealin........................................................ 7

Aurora B kinase.................................................... 9

Histone phosphorylation marks guide the CPC to enrich at the inner

centromeres......................................................... 13

Spindle checkpoint signaling and Aurora B.................................15

Kinetochore-microtubule attachment error-correction .........................16

Microtubules and Microtubule associated protein End-Binding protein 1

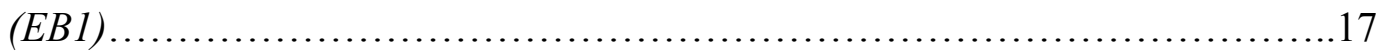

End Binding protein 1 (EB1) .........................................................18 


\section{CHAPTER II: EB1 AND MICROTUBULES TRIGGER THE HISTONE PHOSPHORYLATION PATHWAYS TO ENRICH AURORA B TO THE CENTROMERES IN PROMETAPHASE}

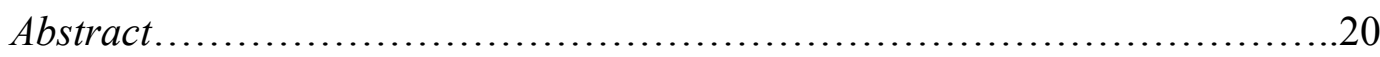

Introduction .......................................................... 21

Results.........................................................24

EB1 regulates Histone phosphorylations to recruit the $C P C$

to centromeres and phosphorylate kinetochore substrates.

EB1 localizes Aurora B to the centromeres in a microtubule

dependent manner............................................... 30

Microtubules stimulate the recruitment of Aurora B to inner

centromeres..................................................... 35

Microtubules cooperate with the histone phosphorylation

pathways to recruit Aurora B to inner centromeres......................36

EB1 interacts with Aurora B at the centromeres in prometaphase........41

Aurora B interacts with K-fibers, PreK-fibers and astral microtubules

in mitosis...........................................................46

EB1 dependent localization of Aurora B to centromeres in

prometaphase is required to phosphorylate kinetochore and

chromatin substrates.............................................51

Aurora B activity on Chromatin is regulated by microtubules

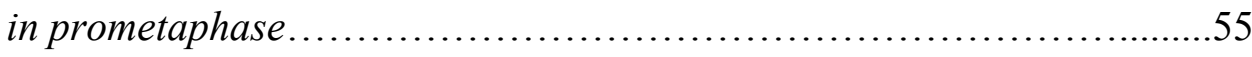

Interdependence of kinetochore axis and cohesion axis through

Aurora B.........................................................63 
EB1 is required for the Aurora B anaphase gradients... .66

Discussion .70

Microtubules/EB1 allow the CPC to communicate with the spindle......72

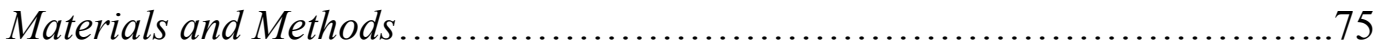

Kinase activity assay ............................................ 75

Cell lines and Plasmids............................................. 75

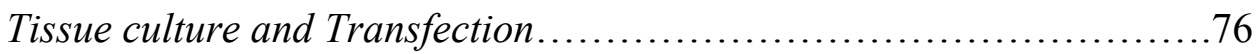

Immunoblotting .............................................. 77

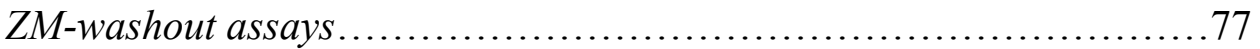

Proximity Ligation Assay .......................................... 78

Immunofluorescence............................................79

Fluorescence Microscopy, Image Acquisition and Processing..............79

Acknowledgments....................................................82

Abbreviations List.......................................................... 83

\section{CHAPTER III: IN VITRO BIOCHEMICAL CHARACTERIZATION OF THE CHROMOSOMAL PASSENGER COMPLEX}

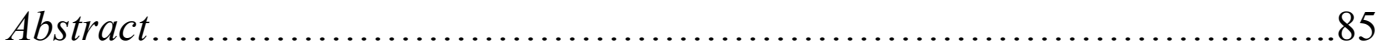

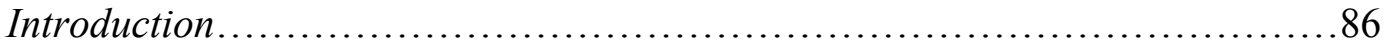

Divergent $N$-terminal region in Aurora kinases.......................86

Co-factors that regulate Aurora B kinase activity.....................87

Results...............................................................90

Purification of the Chromosome passenger complex from E. coli........90

Auto-phosphorylation by Aurora B regulates specific activity of the

$C P C$ 
Cofactors that stimulate Aurora B activity..........................96

Monopolar spindle 1(Mps1)................................96

Centromere targeting sub-unit of CPC (ISD).................97

DNA and double strand breaks............................97

Microtubules...................................................98

Autophosphorylation of Aurora B's $N$-terminus positively and

negatively regulates kinase activity...............................101

CPC-microtubule interaction and auto-regulation by kinase activity....106

Discussion

Aurora B N-terminus has an internal off-switch.....................115

Microtubule interaction and its regulation by kinase activity...........115

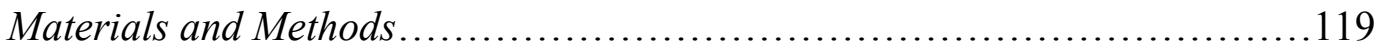

Cloning and site-directed mutagenesis............................119

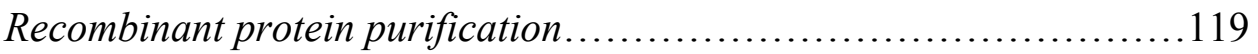

Chromosome passenger complex $(C P C) \ldots \ldots \ldots \ldots \ldots \ldots \ldots \ldots$

FL-AuroraB:INCENP ${ }^{790-856}$ and FL-AuroraB:INCENP ${ }^{790-847} \ldots .120$

Microtubule polymerization....................................121

Microtubule cosedimentation assay................................121

Lambda-Phosphatase treatment..................................121

Kinase activity assay ........................................... 122

Electron Microscopy...........................................122

Acknowledgments................................................ 124 


\section{CHAPTER IV: GENERAL DISCUSSION AND FUTURE DIRECTIONS}

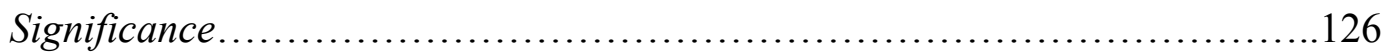

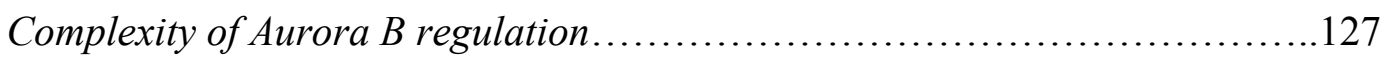

CPC in late mitosis..................................................130

Does EB1-Aurora B regulate chromosome oscillations in metaphase?..............131

CPC localization to centromeres and the role of phosphatases.................134

REFERENCE LIST .................................................... 135

APPENDIX ............................................................... 149

Cell Lines......................................................... 150

Plasmid Maps....................................................... 151 


\section{LIST OF FIGURES}

FIGURE 1-1: SCHEMATIC REPRESENTATION OF CHROMOSOME PASSENGER

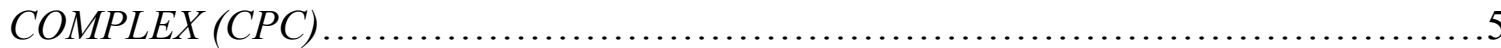

FIGURE 1-2: CRYSTAL STRUCTURE OF HUMAN SURVIVIN WITH THE NTERMINAL HISTONE H3 TAIL PHOSPHRORYLATED ON THR3 .................... FIGURE 1-3:CHROMOSOMAL PASSENGER COMPLEX LOCALIZATION AND FUNCTION DURING MITOSIS.

FIGURE 1-4: TWO HISTONE-PHOSPHORYLATION MARKS ENABLE CPC ENRICHMENT AT INNER CENTROMERE DURING PROMETAPHASE. .14

FIGURE 2-1: EB1 LOCALIZES AURORA B TO CENTROMERES TO PHOSPHORYLATE KINETOCHORES

SUPPLEMENTAL FIGURE 2-1: PHENOTYPES OF EB1 DEPLETION .29

FIGURE 2-2: EB1 LOCALIZES AURORA B AT CENTROMERE IN A MICROTUBULE DEPENDENT MANNER.

SUPPLEMENTAL FIGURE 2-2: ADDITIONAL APPROACHES TO RESCUE EB1 DEPLETION EFFECTS ON CENTROMERIC AURORA B AND COMPARE IT TO MICROTUBULE PERTURBATION. .34

FIGURE 2-3: EB1/ MICROTUBULES ACT UPSTREAM OF CANONICAL CPC LOCALIZATION PATHWAYS.

SUPPLEMENTAL FIGURE 2-3: CENTROMERIC AURORA B IS REDUCED BY REVERSINE OR 5-IODOTUBERICIDINE (HI) TREATMENT IN HELA CELLS ......40 FIGURE 2-4: EBI IS IN CLOSE PROXIMITY TO AURORA B AT CENTROMERES...44 
SUPPLEMENTAL FIGURE 2-4: THE BULK OF EB1 AND CPC LOCALIZE TO DISTINCT SUB-CELLULAR DOMAINS BUT INTERACT NEAR THE KINETOCHORES IN PROMETAPHASE .......................................................... 45 FIGURE 2-5: AURORA B INTERACTS WITH DISTINCT CLASSES OF SPINDLE MICROTUBULES.

FIGURE 2-6: EBI AND MICROTUBULES ARE REQUIRED FOR AURORA B TO PHOSPHORYLATE KINETOCHORES AND CHROMATIN SUBSTRATES IN

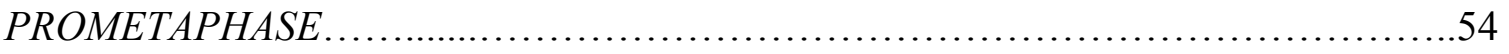
FIGURE 2-7: AURORA B ACTIVITY ON CHROMATIN IS REGULATED BY MICROTUBULES IN PROMETAPHASE, BUT NOT IN PROPHASE ..................58 FIGURE 2-8: CPC ACTIVITY SPREADS FROM CENTROMERES TO CHROMOSOME ARMS AFTER NUCLEAR ENVELOPE BREAKDOWN ............................60 SUPPLEMENTAL FIGURE 2-5: MICROTUBULES REGULATE SPREADING AURORA B ACTIVITY FROM CENTROMERES TO CHROMOSOME ARMS .62 FIGURE 2-9: KINETOCHORE AXIS AND COHESION AXIS ARE CONNECTED THROUGH CENTROMERIC AURORA B .......................................66 FIGURE 2-10: EB1 DEPLETION DISRUPTS AURORA B ANAPHASE GRADIENT....68 FIGURE 2-11: COOPERATION OF HISTONE PHOSPHORYLATION AND MICROTUBULES IN CENTROMERIC LOCALIZATION OF AURORA B..............69 FIGURE 3-1: PURIFICATION AND AUTO-ACTIVATION OF CPC ...................94 FIGURE 3-1: PURIFICATION AND AUTO-ACTIVATION OF CPC (contd.)............95 FIGURE 3-2: COFACTORS REGULATING CPC ACTIVITY/AUTO-ACTIVATION...100 FIGURE 3-3: N-TERMINAL TAIL AUTO-REGULATES AURORA B KINASE. .104 
FIGURE 3-4: ALIGNMENT SHOWING DIVERGENCE IN N-TERMINAL DOMAIN OF XENOPUS AURORA KINASES.......

FIGURE 3-5: MICROTUBULE INTERACTION AND AUTO-REGULATION BY CATALYTIC DOMAIN OF CPC. 110 FIGURE 3-6: MICROTUBULE BINDING ASSAY WITH AI ${ }^{790-856}$ MUTANTS SHOWS INVERSE RELATIONSHIP BETWEEN KINASE ACTIVITY AND MICROTUBULE AFFINITY

FIGURE 3-7: SURFACE CHARGE DISTRIBUTION OF AI ${ }^{790-847}$ SHOWS POSITIVELY

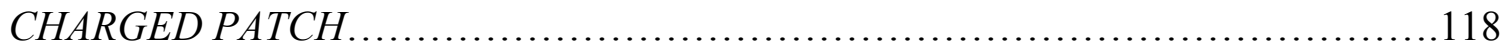
FIGURE 4-1: COMPLEXITY OF AURORA B REGULATION .........................129 FIGURE 4-2: A MODEL FOR HOW DIFFERENTIAL PHOSPHORYLATION OF SISTER KINETOCHORES EMANATES FROM THE POLYMERIZATION STATE OF THE END-ON BOUND MICROTUBULE. 


\section{List of Abbreviations}

APC/C: Anaphase Promoting Complex (cyclosome)

ATP: Adenosine Triphosphate

Bub: Budding uninhibited by benzimidazoles

CPC: Chromosomal Passenger Complex

CENP: Centromere protein (followed by its single-letter designation)

Da: Dalton (unified atomic mass unit, u), equal to $1.66 \times 10^{-27} \mathrm{~kg}$

DNA: Deoxyribonucleic acid

EB1: End Binding Protein 1

EDTA: Ethylene-diamine-tetra-acetic acid

EM: Electron microscopy

GDP: Guanosine diphosphate

GTP: Guanosine triphosphate

H2AT120: Histone H2A threonine 120

H3T3: Histone H3 threonine 3

HI: Haspin Inhibitor

IF: Immuno-fluorescence

IP: Immuno-precipitation

IPTG: Isopropyl $\beta$-D-1-thiogalactopyranoside

ITC: Isothermal Calorimetry

KT: Kinetochore

Mad: Mitotic arrest deficient 
MBP: Myelin Basic Protein

MT: Microtubules

MW: Molecular Weight

PAGE: Polyacrylamide gel electrophoresis

RBS: Ribosome binding site

S: Svedberg's unit (unit for sedimentation rate)

SAC: Spindle assembly checkpoint

SDS: Sodium dodecyl sulfate

ZM: ZM447439, a small molecule inhibitor of human Aurora B kinase 
Chapter I:

GENERAL INTRODUCTION 


\section{Mitosis and Chromosomal Passenger Complex}

Maintenance of a stable genome depends on equal distribution of replicated genetic material during each cell division. In eukaryotes, it is achieved by a highly conserved process called mitosis. German researcher and anatomist Walther Flemming began his pioneering study of mitosis more than 150 years ago (Paweletz, 2001). Cells undergo profound changes in their structure and a series of events are orchestrated with very high precision and precise timing during mitosis. Errors in this process lead to unequal segregation of genetic material or aneuploidy, a condition that is associated with tumorigenesis and birth defects (Boveri, 1914; Ganem et al., 2007). Over the course of mitosis self-organization of the mitotic spindle machinery aligns the chromosomes to the center of the cell which is followed by segregation of sister chromatids to the poles. Cytokinesis commences to generate two daughter cells.

\section{Mitotic Stages}

After replicating the genetic material the cells prepare for mitosis in G2 phase. Cells enter mitosis when they attain a critical concentration of CyclinB/CDK1 activity (Porter and Donoghue, 2003; Sha et al., 2003). This stage, called prophase, is defined by gross changes in chromatin morphology where the genetic material is packaged into condensed chromosomes. Centrosomes, which are the microtubule organizing centers (MTOC), migrate to opposite ends of the nucleus and prepare to form the bipolar spindle (Varmark, 2004). Nuclear envelope breakdown marks the prophase-prometaphase boundary, although some species can undergo mitosis with their nuclear envelope intact (De Souza and Osmani, 2007). Large multi-protein complexes called kinetochores are 
assembled around centromeres on every chromosome in prometaphase. During this stage kinetochores start forming microtubule attachments that initiate the chromosome congression process (Maiato et al., 2004). Successful congression leads to bi-orientation of chromosomes, where each sister chromatid is attached to opposite poles through the kinetochores, that form a compact structure referred to as the metaphase plate. Proper kinetochore-microtubule attachment coupled with centromeric cohesion between the sister chromatids allows tension to build within the mitotic spindle (Gruber et al., 2003). This process is completely self-organized and highly orchestrated. It is important to mention here that cells do not progress further until all chromosomes are aligned at the metaphase plate (Rieder et al., 1994). This is called the spindle checkpoint, and I will discuss this in further detail in the following section. Upon satisfying the spindle checkpoint CyclinB is degraded by the anaphase promoting complex/cyclosome (APC/C) ubiquitin ligase, which initiates a cascade of events that leads to loss of sister chromatid cohesion (Nasmyth, 2002). This coupled with microtubule depolymerization results in separation of the sister chromatids and their migration towards the pole. This stage is called anaphase, which is followed by cytokinesis where the furrow divides the cell into two daughter cells. Finally, in telophase the cytokinetic furrow ingression is completed, the cell forms a nuclear envelope around the acquired copy of genome and the chromatin decondenses.

In mammals this whole process takes about an hour and every component involved in it works in a perfectly orchestrated manner. It is analogous to a classical symphony of which Aurora B plays the role of the 'conductor' (Ruchaud et al., 2007). 


\section{Aurora B and its fellow passengers}

Aurora kinase was first discovered in Drosophila where mutation in this gene resulted in a failure of centrosome separation generating monopolar spindles, hence it was given the name "Aurora," reminiscent of the North Pole (Glover et al., 1995). Since then homologues of Aurora have been identified in different species (Adams et al., 2000; Chan and Botstein, 1993; Schumacher et al., 1998). Aurora B is the catalytic subunit of a four protein complex called the 'Chromosomal passenger complex' (CPC), where the three non-catalytic subunits are INCENP, survivin and Dasra/ Borealin (Cooke et al., 1987; Earnshaw and Cooke, 1991; Carmena and Earnshaw, 2003; Vagnarelli and Earnshaw, 2004; Vader et al., 2006; Terada et al., 1998; Adams et al., 2000; Honda et al., 2003; Sampath et al., 2004; Gassmann et al., 2004; Nakajima et al., 2009; Bolton et al., 2002). The in vivo localization of the CPC proteins was first observed with a monoclonal antibody against 'inner centromeric protein' INCENP (Earnshaw and Cooke, 1991). It showed chromosome localization in prometaphase/metaphase and subsequent re-location to the spindle midzone and the equatorial cell cortex. It was proposed that these proteins used the chromosomes as a conveyance to reach the central region of the cell and hop off to the spindle midzone / cortex, hence the name 'chromosomal passenger' (Earnshaw and Bernat, 1991). 


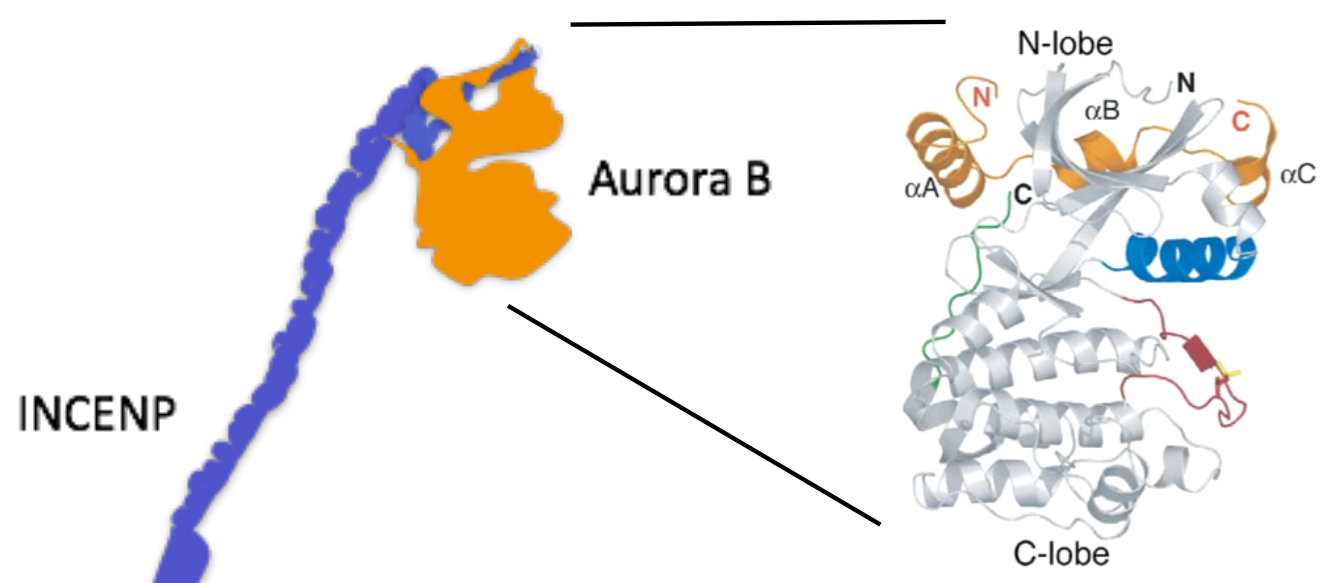

Survivin
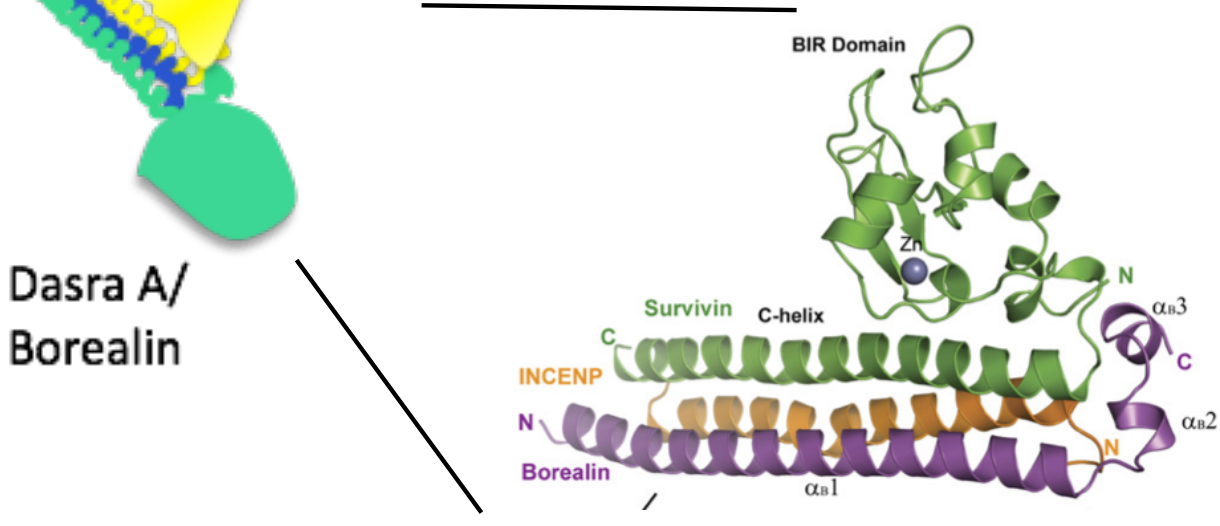

Figure 1-1: Schematic representation of Chromosome passenger complex (CPC) along with available crystal structures of centromeric targeting domain and catalytic domain. (Jeyaprakash et al., 2007; Sessa et al., 2005) 
INCENP

INCENP was the first member to be identified that came out of a screen for new components on the mitotic chromosome (Cooke et al., 1987). It is a scaffold that unites survivin and Borealin with Aurora B (Klein et al., 2006; Jeyaprakash et al., 2011) through a triple helical interaction with Borealin and survivn with its N-terminus and Aurora B interaction through a highly conserved C-terminal fragment called 'IN-Box' (Figure 1-1) (Adams et al., 2000; Sessa et al., 2005; Jeyaprakash et al., 2007). It has a central coiled coil domain that has microtubule binding activity which is heavily regulated by Cdk1 and Aurora B phosphorylation (Mackay et al., 1998; Nakajima et al., 2011). INCENP is also a cofactor that stimulates Aurora B activity through its C-terminal interaction (Bishop and Schumacher, 2002; Adams et al., 2000). Phosphorylation of 'TSS' motif in INCENP by Aurora B is critical kinase activation (Bishop and Schumacher, 2002; Sessa et al., 2005; Honda et al., 2003). This interaction has some similarity with the interaction between another Aurora kinase, Aurora A, and a microtubule associated protein TPX2 (Bayliss et al., 2003; Tsai et al., 2003; Eyers et al., 2003). This is discussed in further details in the following chapters. Overall, INCENP is a key regulator of both activity and localization of Aurora B kinase.

\section{Survivin}

Survivin is a conserved member of the CPC, found in yeast through humans. It is a member of the Inhibitor of Apoptosis (IAP) family, which has a single baculovirus IAP repeat (BIR) domain that can dimerize (Ambrosini et al., 1997; Chantalat et al., 2000). Its anti-apoptotic role is less well characterized. We will focus on its role as a member of the 
CPC. Although survivin is known to dimerize, it interacts with Borealin and INCENP as a monomer (Jeyaprakash et al., 2007). Survivin is phosphorylated by Aurora B in vitro (Bolton et al., 2002; Wheatley et al., 2004). Ubiquitination of survivin regulates CPC dynamics at the centromeres (Vong et al., 2005). The key role of survivin is recognition of phosphorylated histone $\mathrm{H} 3 \mathrm{Thr} 3$ in prometaphase that is essential in recruiting $\mathrm{CPC}$ to centromeres (Wang et al., 2010; Kelly et al., 2010; Yamagishi et al., 2010). It does so by the S3 pocket of its BIR domain, where histidine 80 and lysine 62 allows docking of CPC on the phosphorylated threonine 3 of histone H3 (Figure 1-2) (Niedzialkowska et al., 2012; Jeyaprakash et al., 2011).

\section{Dasra / Borealin}

Borealin was identified in a proteomic screen for new components on the mitotic chromosomes (Gassmann et al., 2004) and simultaneously in another screen in Xenopus looking for chromosome binding proteins, where it was named Dasra-B (Sampath et al., 2004). It forms a triple-helical interaction with INCENP and survivin critical for centromeric recruitment of CPC (Klein et al., 2006; Jeyaprakash et al., 2007; Wang et al., 2010; Yamagishi et al., 2010; Kelly et al., 2010). Mps1, a mitotic kinase, phosphorylates Borealin to regulate its dimerization state and this phosphorylation is shown to be required for Aurora B activity (Jelluma et al., 2008; Bourhis et al., 2009). Dasra has been shown to bind plasmid coated chromatin beads that suggest it might also have a DNA binding domain but this role is less well characterized (Kelly et al., 2007). 


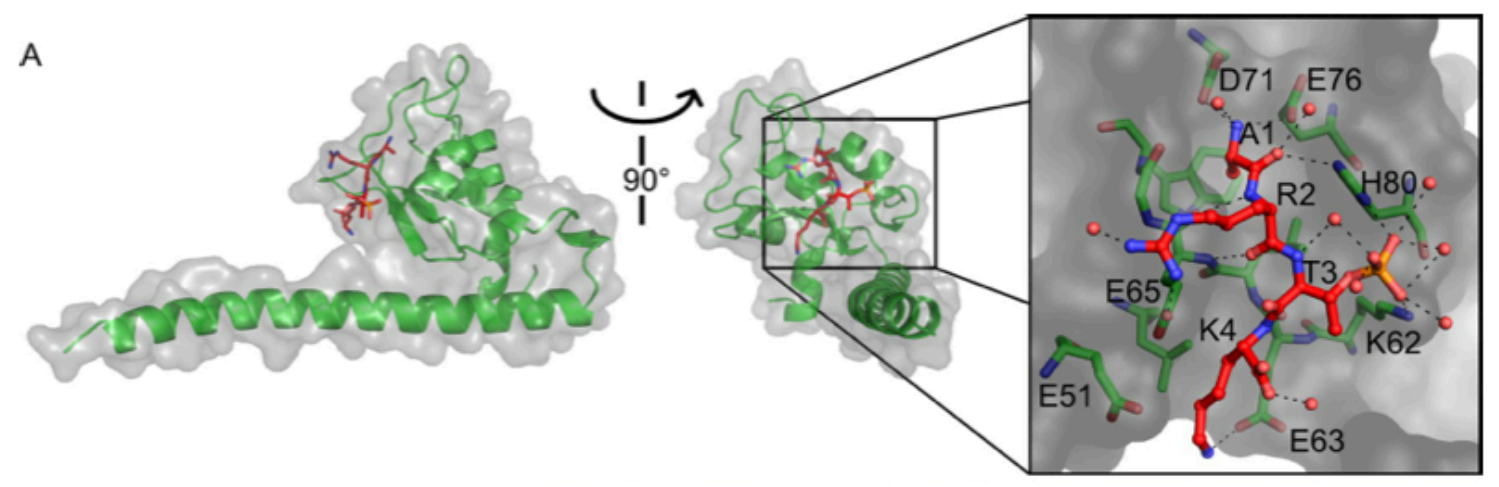

Figure 1-2: Crystal structure of human Survivin with the $\mathrm{N}$-terminal histone H3 tail phosphrorylated on Thr3.

Two orientations of Survivin rotated $90^{\circ}$ relative to each other. Survivin is shown in green and surface density shown in gray. Histone $\mathrm{H} 3$ peptide is shown in red. Zoom in view shows residues of survivin interacting with H3pT3 (orange-phosphate). H80 and K62 are key residues that allow survivin docking on phospho-Thr3. (Niedzialkowska et al., 2012). 


\section{Aurora B kinase}

Aurora kinases are serine/threonine kinase and are conserved from S.cerevisiae to humans. In vertebrates there are 3 Aurora kinases - A, B and C (Adams et al., 2001b) and each has distinct function and tissue specificity (Carmena and Earnshaw, 2003). As explained earlier Aurora B activity is regulated by many cofactors, INCENP being the most important one (Bishop and Schumacher, 2002; Sessa et al., 2005; Honda et al., 2003; Rosasco-Nitcher et al., 2008; Jelluma et al., 2008; Fuller et al., 2008). It is overexpressed in many solid tumors (Bischoff et al., 1998; Giet and Prigent, 1999). Aurora $\mathrm{C}$ can bind other members of the complex and can rescue Aurora B loss of function in several human cell lines (Yan et al., 2005; Sasai et al., 2004). The dynamic localization of $\mathrm{CPC}$ during mitosis allows Aurora B to phosphorylate many substrates on chromatin, at the inner centromere, the kinetochore and the spindle midzone. Histone H3S10 is a well-known chromatin-associated Aurora B substrate (Hsu et al., 2000; Adams et al., 2001a; Murnion et al., 2001; Crosio et al., 2002). MCAK, a kinesis-13 family member and a MT depolymerase is phosphorylated by Aurora B at centromeres on several residues (Wordeman and Mitchison, 1995; Hunter et al., 2003; Andrews et al., 2004; Lan et al., 2004; Kline-Smith et al., 2004). These phosphorylations regulate localization and function of MCAK (Lan et al., 2004; Knowlton et al., 2006; Zhang et al., 2007; Ems-McClung et al., 2007). Aurora B also phosphorylates many kinetochore proteins including Ndc80, KNL1, Cep57 and budding yeast Dam1 (Emanuele and stukenberg, 2007; Cheeseman et al., 2002; Deluca et al., 2006). Anaphase substrates of Aurora B include MKLP1, Mgc-Rac-GAP, MCAK, Vimentin, MLCK (Minoshima et al., 
2003; Guse et al., 2005; Lan et al., 2004; Yokoyama et al., 2005). Aurora B directs many different mitotic functions through phospho-regulation of these substrates (Figure 1-3). 
Figure 1-3:Chromosomal passenger complex localization and function during mitosis. Schematic representation of the chromosomal passenger complex (CPC) localization (green) correlated with its multiple functions (grey boxes) and principal targets (red boxes) during the different phases of mitosis relative to tubulin and chromosome dynamics. In prophase, the CPC is found on chromosome arms where it phosphorylates histone H3 on Ser10 and Ser28. It is involved in the release of arm cohesion and mitotic chromosome structure. During this phase it accumulates at centromeres where the maturation of kinetochores begins and continues through prometaphase. The CPC is required for the formation of a bipolar spindle and its stability from prophase/prometaphase to anaphase. In metaphase, it localizes at centromeres, where it has a central role in centromeric cohesion and the regulation of kinetochore-microtubule attachments. It controls the correct alignment of chromosomes on the spindle equator and the spindle checkpoint. In anaphase, the CPC translocates to the spindle midzone and appears at the cortex; it is involved in the formation of the central spindle. In telophase, the $\mathrm{CPC}$ concentrates at the cleavage furrow and, subsequently, at the midbody, where it is required for completion of cytokinesis. Chromosomes, blue; tubulin, red; nuclear envelope, grey. CENP-A, centromere protein-A; CYK-4, CYtoKinesis defect (Caenorhabditis elegans MgcRacGAP homologue); EVI5, ecotropic viral integration site-5; GFAP, glial fibrillary acidic protein; HEC1, highly expressed in cancer-1; MgcRacGAP, Rac GTPase activating protein-1; MCAK, mitotic centromere-associated kinesin; MKLP1, mitotic kinesin-like protein-1; Ndc80, yeast homologue of HEC1; ZEN-4, Zygotic epidermal ENclosure defective (C. elegans MKLP1 homologue). (Ruchaud et al., 2007) 


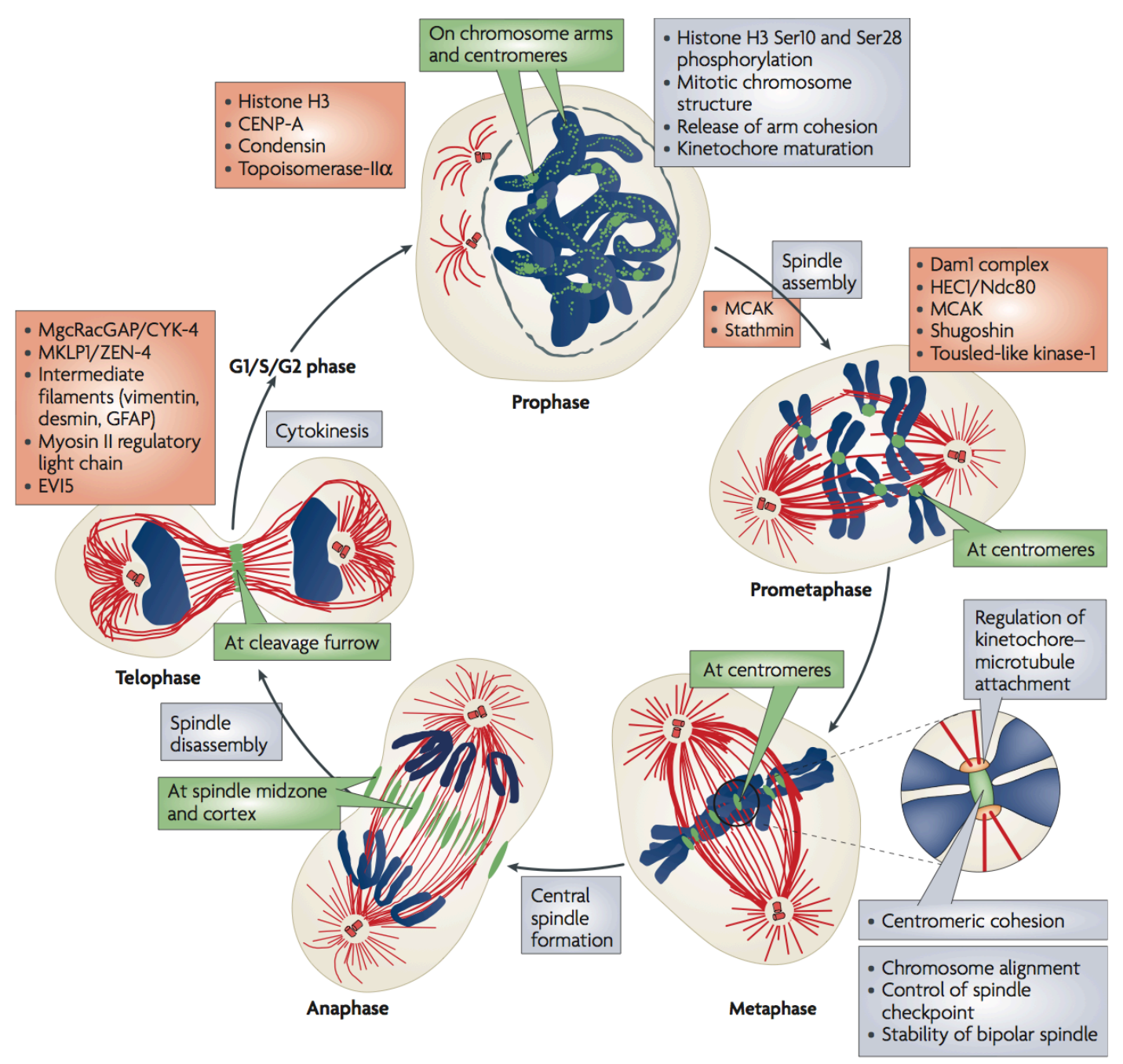

Figure 1-3 


\section{Histone phosphorylation marks guide the CPC to enrich at the inner centromeres.}

The CPC is detected on the chromosome arms in prophase and then it gradually concentrates at the inner centromere in prometaphase (Earnshaw and Cooke, 1991). Recent studies from three groups showed that CPC enrichment at the inner centromeres are guided by two phospho-histone marks. Haspin kinase phosphorylates histone H3 on threonine 3 which is recognized by survivin to localize CPC to the inner centromeres (Wang et al., 2010; Kelly et al., 2010; Jeyaprakash et al., 2011; Du et al., 2012; Niedzialkowska et al., 2012). Bub1, recruited in a Mps1 dependent manner, phosphorylates histone $\mathrm{H} 2 \mathrm{~A}$ on threonine 120 that is recognized by Shugoshin (Sgo1) which recruits CPC (Yamagishi et al., 2010). The phospho-histone H3Thr3 marks exist along the margin between the sister-chromatids, which is referred to as the cohesion axis, and the phopsho-histone H2AThr120 mark is high at the kinetochores but extends into the inner centromeres, which we refer to as the kinetochore axis (Figure 1-4). CPC enrichment is the highest at the inner centromeric region where these two axes intersect. Interestingly, both Haspin kinase and Mps1 kinase require Aurora B activity for their function (Wang et al., 2011b; Saurin et al., 2011). These data suggests that these are feedback loops that are triggered by Aurora B activity to allow enrichment of CPC at the centromeres. But what brings the initial pool of CPC to the centromeres remains an interesting question that will be discussed in chapter 2 . 

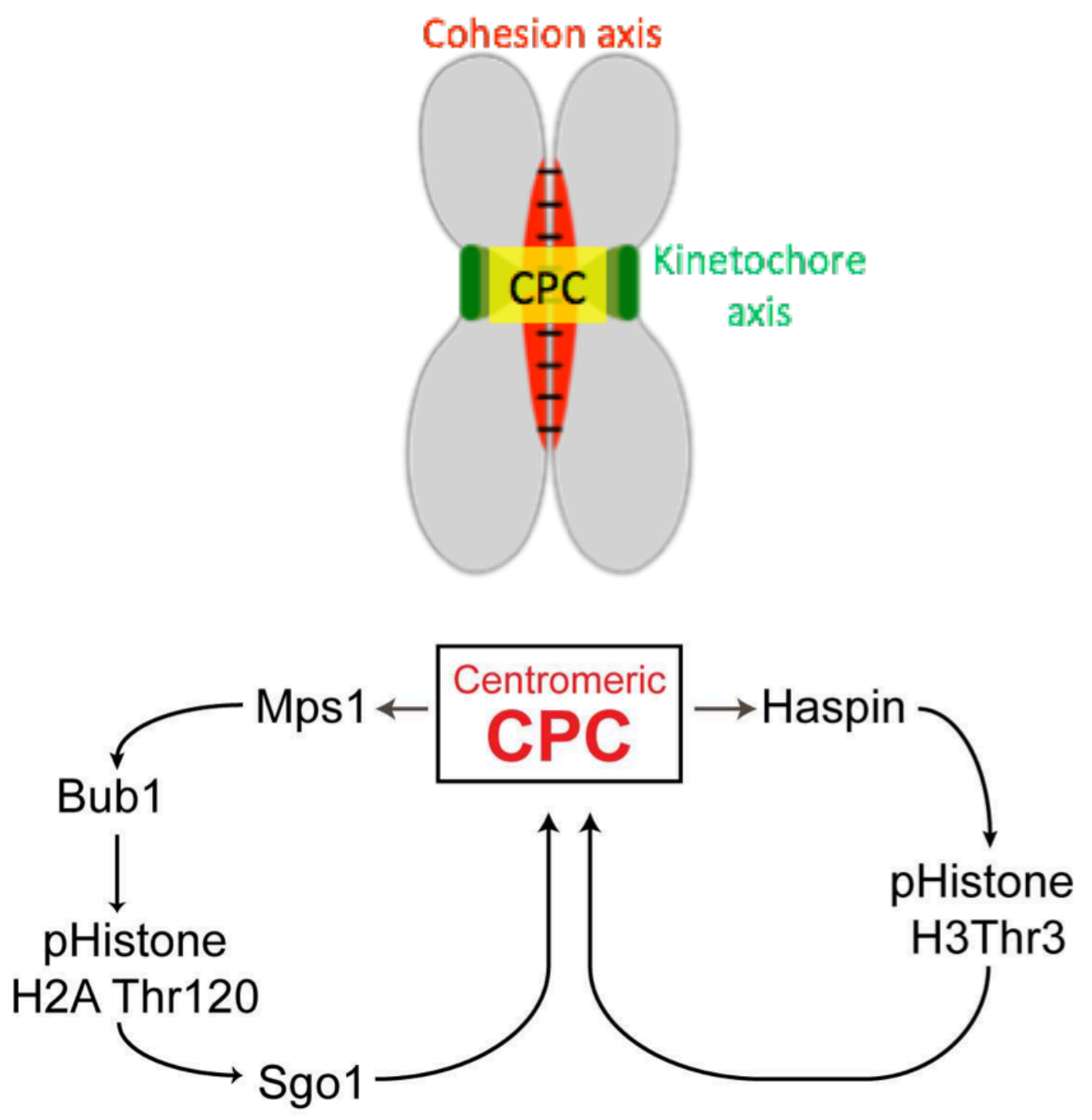

Figure 1-4: Two histone-phosphorylation marks enable CPC enrichment at inner centromere during prometaphase. We refer to the Mps1-Bub1-Sgol axis as kinetochore axis (green) and the Haspin-pH3T3 as the cohesion axis (red). Aurora B activity stimulates both the loops. 


\section{Spindle checkpoint signaling and Aurora B}

As briefly mentioned earlier, only after every chromosome is bi-oriented at the central metaphase plate does a cell go into anaphase. The spindle checkpoint provide the 'wait' signal for halting the cell in metaphase (Rieder et al., 1994; Hartwell and Weinert, 1989). Unattached kinetochores recruit the checkpoint proteins to send out the spindle checkpoint signal which inhibits degradation of Cyclin B by the anaphase promoting complex/cyclosome (APC/C) ubiquitin ligase (Rieder et al., 1995; Reed, 2003; Musacchio and Hardwick, 2002; Nasmyth, 2002). Although this suggests that absence of microtubule attachments is the signal for activating the spindle checkpoint, there is evidence that the spindle can measure tension at each kinetochore-microtubule attachment and lack of tension triggers the checkpoint (Nicklas and Koch, 1969; Nicklas et al., 2001; Matson et al., 2012; Biggins and Murray, 2001). Whether 'occupancy' (or lack of microtubules, i.e. unoccupied kinetochores) and 'tension' are two distinct arms to signaling the checkpoint or whether loss of tension results in microtubule release (and hence, unoccupied kinetochores) remains under intense debate.

The two main group of proteins that constitute the spindle checkpoint are Mad (Mitotic arrest deficient) and Bub (Budding uninhibited by benzimidazole) family of proteins (Li and Murray, 1991; Hoyt et al., 1991). Both were identified through mutagenesis screens in yeast treated with microtubule poisons benomyl and benzimidazole, respectively. But these are not the only proteins involved in the spindle checkpoint. The list is extensive and still expanding. Aurora B activity is required to signal the spindle checkpoint (Kallio et al., 2002; Hauf et al., 2003; Matson et al., 2012). This was first shown in yeast (Biggins and Murray, 2001). It is argued that signaling the 
spindle checkpoint might be a secondary effect of generating unattached kinetochores (Tanaka et al., 2002). Whether Aurora B has a direct role in signaling the spindle checkpoint is intensely debated (Santaguida et al., 2011; Tanaka et al., 2002). Inhibiting Aurora B leads to failure to recruit checkpoint protein BubR1 to kinetochores, missegregation of chromosomes and aberrant mitosis (Kallio et al., 2002; Hauf et al., 2003; Ditchfield et al., 2003). Inner centromeric enrichment of CPC is critical for proper functioning of Aurora B. Treating HeLa cells with a haspin kinase inhibitor leads to loss of centromeric Aurora B and reduced phosphorylation of centromeric and kinetochore substrates. Consequently, chromosome alignment and spindle checkpoint signaling are compromised (Wang et al., 2012).

\section{Kinetochore-microtubule attachment error-correction}

After nuclear envelope breakdown polymerizing microtubule emanating from centrosomes at opposite ends make numerous attempts to make attachments with the kinetochores, a process called 'search and capture' (Tanaka et al., 2005). This frequently results in both kinetochores making connections with microtubule emanating from the same pole, a condition called 'syntelic attachment'. This is an erroneous attachment, as both chromatids will end up in the same daughter cell after anaphase, resulting in aneuploidy. Aurora B resolves such interactions and promotes bi-orientation of chromosome (Adams et al., 2000; 2001b; Kallio et al., 2002; Kaitna et al., 2000). Resolution of syntelic attachments require Aurora B activity (Hauf et al., 2003). Cells treated with monastrol, an Eg5 inhibitor, arrest in mitosis with mono-polar spindles (Mayer et al., 1999), and upon release cells exhibit a high frequency of syntelic 
attachments (Kapoor et al., 2000). Cells treated first with monastrol, and then released into Aurora B inhibitor, ZM447439 and proteosome inhibitor, MG132, to prevent mitotic exit in the absence of Aurora B activity, were unable to correct syntelic attachments (Lampson et al., 2004). Instead, monastrol release into MG132 containing media showed syntelically attached chromosome move rapidly towards the spindle pole, followed by congressing to the metaphase plate (Lampson et al., 2004). These data provide a mechanism for syntelic kinetochore-microtubule attachment resolution, in which improperly attached chromatids must travel to the spindle poles in order to be repaired.

Another form of erroneous kinetochore-microtubule attachment arises when one kinetochore is attached to microtubules from both poles, a condition called 'merotelic attachment' (Cimini et al., 2003). Aurora B is recruited specifically to merotelic attachment points and Aurora B activity is required to resolve merotelic attachments (Knowlton et al., 2006; Cimini et al., 2006).

\section{Microtubules and microtubule associated protein End-Binding protein 1(EB1)}

Although Aurora B kinase can autoactivate in vitro it requires some active kinase to initiate this reaction (Rosasco-Nitcher et al., 2008). Fully inactive Xenopus Aurora B can be activated in vitro by microtubules and a cofactor, TD60/RCC2 (Rosasco-Nitcher et al., 2008) and spindle formation in Xenopus requires both chromatin and microtubule binding activities (Tseng et al., 2010). Moreover, anaphase cells treated with nocodazole for 8 minutes are not phosphorylated on an activating site on INCENP (Fuller et al., 2008). Therefore there are numerous examples of microtubules being a potent cofactor for Aurora B kinase. In chapter 2 we will focus on a subset of microtubules that are 
nucleated from the kinetochores called pre-formed K-fibers (PreK-fibers) (Khodjakov et al., 2003; Platani et al., 2009; Mitchison and Kirschner, 1985), which we find are a unique class of spindle microtubules that regulate the levels of Aurora B at centromeres. Nocodazole washout experiments in GFP-tubulin expressing cells co-stained with Bub1 confirmed that these were indeed kinetochore nucleated microtubules that were kinetically distinct from centrosome nucleated microtubules and were differentially regulated (Tulu et al., 2006).

\section{End Binding protein 1 (EB1)}

EB1 is a microtubule associated protein which bind growing tips of microtubules, and hence belongs to a group of proteins called +TIPS (Morrison et al., 1998; Lansbergen and Akhmanova, 2006). EB1 fails to bind to taxol-stabilized microtubules suggesting that it has affinity for dynamic microtubule ends. EB1 was first identified in a yeast-2-hybrid screen designed to find adenomatous polyposis coli (APC) binding partners ( $\mathrm{Su}$ et al., 1995). EB1 depletion phenotypes include mis-segregation of chromosome or spindle orientation defects arising from altered microtubule dynamics (Green et al., 2005; Lee et al., 2000; Draviam et al., 2006; Morrison et al., 1998). EB1 yeast homologue Bim1p, associates with Ipl-1 (Aurora B) to regulate anaphase spindle dynamics (Zimniak et al., 2009; 2012). Another study reveals that EB1 depletion causes sister chromatid / kinetochore orientation defects without affecting kinetochore-microtubule attachments that results in chromosome mis-segregation (Draviam et al., 2006). EB1 has been also shown to co-immunoprecipitate with Aurora B and regulate its activity by inhibiting PP2A mediated dephosphorylation (Sun et al., 2008). 


\section{CHAPTER II:}

\section{EB1 AND MICROTUBULES TRIGGER THE HISTONE PHOSPHORYLATION PATHWAYS TO ENRICH AURORA B TO THE CENTROMERES IN PROMETAPHASE}

This chapter is based on the following manuscript under review for publication. Budhaditya Banerjee ${ }^{1}$, Cortney A. Kestner ${ }^{1}$ and P. Todd Stukenberg ${ }^{1,2}$ "EB1 and microtubules trigger the histone phosphorylation pathways to enrich Aurora B at centromeres in prometaphase." (under revision at The Journal of Cell Biology) 


\begin{abstract}
The Aurora B kinase coordinates kinetochore-microtubule attachments with spindle checkpoint signaling on each mitotic chromosome. We find that EB1, a microtubule plusend tracking protein, is required to enrich Aurora B at inner centromeres in a microtubule dependent manner. This regulates phosphorylation of both kinetochore and chromatin substrates. EB1's role is independent and upstream of the histone phosphorylation marks (HistoneH2A phospho-Thr120 and HistoneH3 phospho-Thr3) that localize Aurora B. The Chromosomal passenger complex (CPC) containing Aurora B can be found on a subset of spindle microtubules that exist near prometaphase kinetochores, known as pre-formed K-fibers. Our data suggest that EB1 enables the spindle microtubules to regulate the phosphorylation of kinetochores through recruitment of Aurora B kinase.
\end{abstract}




\section{Introduction}

The Chromosome Passenger Complex (CPC) catalytic subunit Aurora B kinase is a central regulator of mitotic events (reviewed(Ruchaud et al., 2007; Knowlton et al., 2006; Cimini et al., 2006). It has distinct roles in different stages of mitosis. In prophase Aurora B phosphorylation is found along the length of mitotic chromosomes where it releases cohesion and phosphorylates Histone $\mathrm{H} 3$ on serine 10 to release the heterochromatin protein 1 (Deluca et al., 2006; Hirota et al., 2005; Welburn et al., 2010;

Fischle et al., 2005; Cheeseman et al., 2006; Hsu et al., 2000; Losada et al., 2002). From late in prophase until the onset of anaphase Aurora B concentrates at inner centromeres where it regulates inner centromere substrates as well as kinetochore microtubule attachments and the spindle checkpoint signal (Ruchaud et al., 2007; Salimian et al., 2011; Lan et al., 2004; Knowlton et al., 2006; Cimini et al., 2006; Kallio et al., 2002; Lampson et al., 2004; Liu et al., 2009). While it is well accepted that the movement of the CPC to distinct locations is critical for its ability to carry out multiple functions in different stages of mitosis (Mishra et al., 2010; Terada, 2001; Orjalo et al., 2006; Wheatley et al., 2001a), it is not known how Aurora kinase is differentially regulated during mitotic progression.

Aurora B kinase regulates spindle checkpoint signaling and the release of improper kinetochore attachments (Mishra et al., 2010; Lan et al., 2004; Knowlton et al., 2006; Cimini et al., 2006; Kallio et al., 2002; Liu et al., 2009). These events must be measured independently on each chromosome. How Aurora B kinase can integrate local information about microtubule attachment status to regulate these chromosome autonomous events is a critical unanswered question. 
The concentration of the $\mathrm{CPC}$ at inner centromeres is mediated by posttranslational modifications of histones. The survivin subunit binds the Histone H3 tails that are phosphorylated at Threonine-3 by haspin kinase (Salimian et al., 2011; Wang et al., 2010; Kelly et al., 2010; Yamagishi et al., 2010; Niedzialkowska et al., 2012; Du et al., 2012; Jeyaprakash et al., 2011). The CPC also interacts with Shugoshin (Sgo1), which is recruited to Histone H2A that is phosphorylated on Threonine-120 by Bub1 (Yamagishi et al., 2010). It is not clear if the presence of these histone marks at inner centromeres are sufficient for CPC localization and there are many reports of additional requirements including survivin phosphorylation (Tsukahara et al., 2010; Wheatley et al., 2004; Chu et al., 2011) and regulation by exportin binding and nuclear pore proteins (Knauer et al., 2006; Platani et al., 2009).

EB1 is a microtubule 'plus-end' tracking protein that interacts with growing tips of microtubules (Morrison et al., 1998) and its yeast homologue Bimlp forms a stable association with Ipl-1/Aurora B to regulate anaphase spindle morphology (Zimniak et al., 2009). Furthermore, EB1 has been shown to co-immunoprecipitate with Aurora B and regulate its activity by inhibiting PP2A mediated dephosphorylation (Sun et al., 2008). However, the biological significance of this interaction remains unclear.

The microtubule associated protein TPX2 and microtubules have been shown to activate the related kinase, Aurora A in mitosis. High Ran-GTP on condensed chromosomes releases TPX2 from importin-bound inactive state, which then binds Aurora A and protects the T-loop from dephosphorylation by PP1 in a microtubule dependent manner (Tsai et al., 2003; Bayliss et al., 2003). Microtubules also regulate Aurora $\mathrm{B}$ activity. The $\mathrm{CPC}$ binds microtubules in two distinct regions. INCENP has a 
central coiled coil region that can bind microtubules (Mackay et al., 1998) and Aurora B bound to the IN-box can also bind microtubules (Rosasco-Nitcher et al., 2008). Although Aurora B kinase can auto-activate in vitro it requires some active kinase to initiate this reaction (Rosasco-Nitcher et al., 2008). Fully inactive Xenopus Aurora B can be activated in vitro by microtubules and a cofactor, TD60/RCC2 (Rosasco-Nitcher et al., 2008) and spindle formation in Xenopus requires both chromatin and microtubule binding activities (Tseng et al., 2010). Moreover, anaphase cells treated with nocodazole for 8 minutes are not phosphorylated on an activating site on INCENP (Fuller et al., 2008). However, microtubules are absent in prophase nuclei that have Histone H3 phosphorylated on Ser10. It is also unclear if the CPC is regulated by microtubules in prometaphase since the CPC proteins do not localize primarily to microtubules at this time and Aurora B kinase activity can be measured in mitotic cells arrested by spindle poison nocodazole.

Here we demonstrate that the $\mathrm{CPC}$ at the inner centromere is substantially enriched by microtubules near the kinetochore by a novel pathway that requires the EB1 plus end tracking protein. There is a similar EB1/microtubule dependent increase in phosphorylation of Aurora B substrates at kinetochores and chromosome arms. The regulation by $\mathrm{EB} 1 /$ microtubules is upstream and independent of the histone phosphorylation pathways that localize the CPC. We show that microtubules in PreKfiber bundles contain Aurora B and can enrich Aurora B at inner centromeres. These findings establish a new prometaphase pathway regulating Aurora B localization that requires $\mathrm{EB} 1 /$ microtubules and provides a new mechanism for the spindle to regulate CPC activity. 


\section{Results}

EB1 regulates Histone phosphorylations to recruit the CPC to centromeres and phosphorylate kinetochore substrates.

We asked whether Aurora B phosphorylation of kinetochore substrates in prometaphase required EB1. HeLa cells were depleted of EB1 using either a coding sequence targeted siRNA (EB1siRNA) or a combination of two EB1 siRNAs targeted to 3'-untranslated region (siEB13UTR) (Suppl. Fig. 2-1 A). Kinetochore protein KNL1 phosphorylation by Aurora B was measured using a phospho-KNL1 (pKNL1) antibody. The antibody recognized pKNL1 at kinetochores but also cross reacted with a centrosome protein as previously shown (Welburn et al., 2010). We specifically quantified kinetochores from prometaphase cells because metaphase aligned chromosomes show reduced KNL1 phosphorylation (Welburn et al., 2010). KNL1 phosphorylation at prometaphase kinetochores was significantly reduced in cells depleted of EB1 with either set of siRNAs (Fig. 2-1 A,B and Suppl. Fig. 2-2 A). KNL1 protein levels were not reduced in EB1 depleted HeLa cells (Fig. 2-2 E). Surprisingly, inner centromeric Aurora B levels were also reduced in EB1 depleted prometaphase cells (Fig. 2-1 A,C and Suppl. Fig. 2-2 A,B). There was a similar drop in two other CPC proteins Borealin/Dasra (Borealin) and INCENP at the inner centromeres suggesting that EB1 is required to recruit the whole CPC complex (Suppl. Fig. 2-2 D-F). Aurora B, INCENP and Survivin protein levels in EB1 depleted cells were similar to control HeLa cells so the depletion from centromeres was not due to destabilization of CPC proteins (Suppl. Fig. 2-1 E).

EB1 depletion also reduced both of the histone marks that recruit Aurora B to inner centromeres. Cells depleted of EB1 had reduced levels of Histone H2A phospho-Thr120 
(pH2AT120 in figures) and HistoneH3 phospho-Thr3 (pH3T3 in figures) as measured by immunofluorescence with phospho-specific antibodies (Fig. 2-1 D, F and G and Suppl. Fig. 2-1 B). Bub1 kinase levels were also reduced at the kinetochores of EB1 depleted cells (Fig. 2-1 E and H). HEK293T cells also reduced phospho-KNL1, Aurora B, Bub1 and phospho-Histone H2AThr120 levels after EB1 depletion (Suppl. Fig. 2-1 C,D). We conclude that EB1 is required to generate the phospho-histone marks that recruit the CPC to phosphorylate kinetochores. 
Figure 2-1: EB1 localizes Aurora B to centromeres to phosphorylate kinetochores

A) HeLa cells depleted of EB1 were immunostained with anti-phospho-KNL1(Ser60) (pKNL-1) and Aurora B antibodies. Bar-2 $\mu \mathrm{m}$. B) Quantification of immunostaining intensities of pKNL1 shown in A. * p-value $=2.0 \mathrm{e}^{-127}$. C) Centromeric Aurora B levels with dots depicting intensities outside $5-95^{\text {th }}$ percentiles ( $>300$ centromeres). $*$ pvalue $=1.2 \mathrm{e}^{-105}$. D) EB1 depletion reduces Bub1 mediated phosphorylation of HistoneH2A (pH2AT120). Bar-1.6um. E) EB1 depletion reduces Bub1 at kinetochores. Bar-2.2um. Quantification of pH2AT120 (F), phospho-Histone H3Thr-3 (pH3T3) (G) and Bub1 (H) levels in control and EB1 depleted HeLa cells. (See Suppl. Fig. 2-1 B for pH3T3 staining examples) 
A
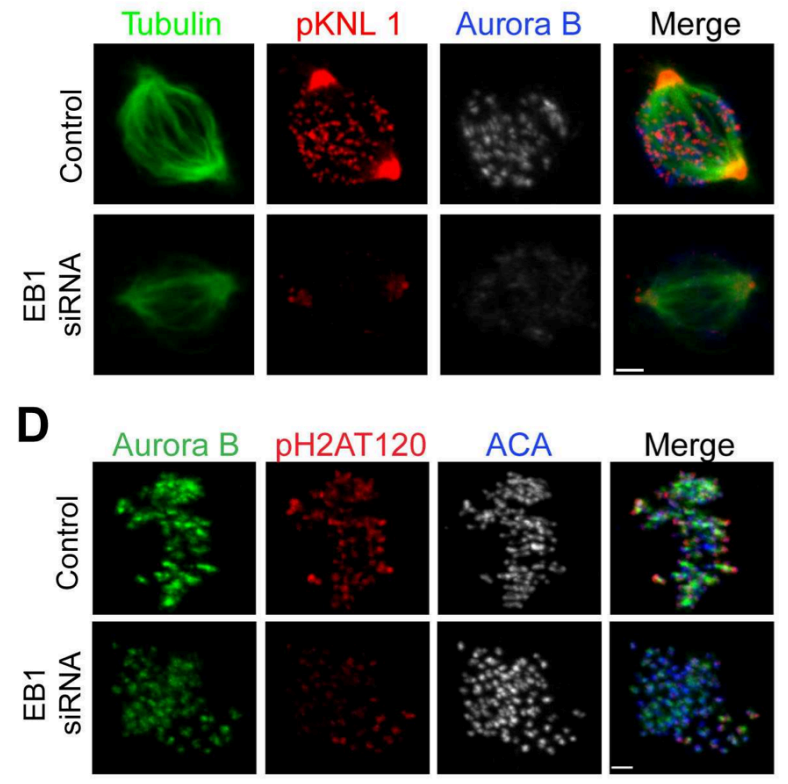

E
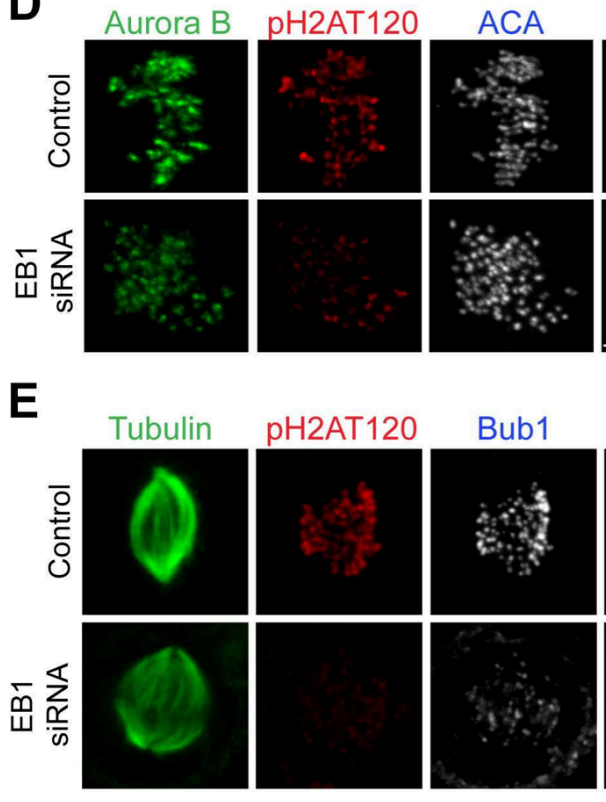

Bub1

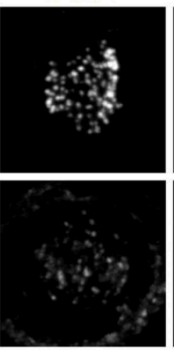

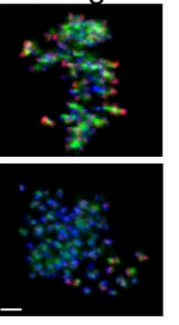

Merge

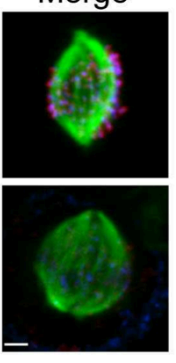

Merge
B

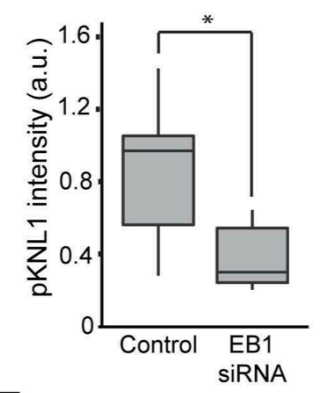

F
C

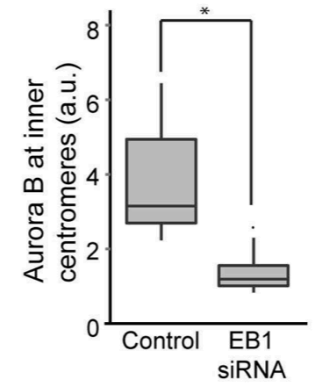

G

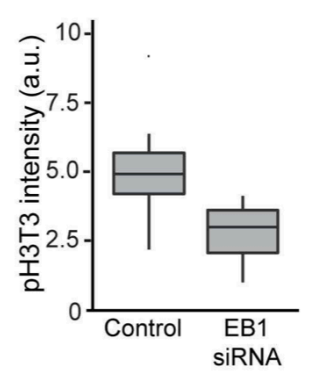

Figure 2-1 


\section{Supplemental Figure 2-1: Phenotypes of EB1 depletion.}

A) Protein levels as measure by immunoblot of experiment in Fig. 2-1. HeLa cells treated with either a siRNA targeting the EB1 coding sequence (EB1 siRNA $-10 \mu \mathrm{M})$ or a combination of two EB1-3'UTR targeted siRNAs (siEB13UTR - 10nM each) for 48 hours. B) Immunostaining of EB1 depleted HeLa cells with anti-phosho-Histone H3Thr3 (pH3T3) antibodies. Bar $=1.9 \mu \mathrm{m} . \mathbf{C}$ and D) EB1 depletion in HEK293T cells gives the same phenotype as HeLa cells. HEK293T cells were depleted as in A and protein levels measured (C). D) EB1 depletion in HEK293T cells shows reduced levels of Aurora B at the inner centromere, reduced Bub1 at kinetochores and reduced phospho-KNL1 levels. E) Immunoblot to compare CPC proteins - INCENP, Aurora B and survivin in control depleted and EB1 depleted cells. 


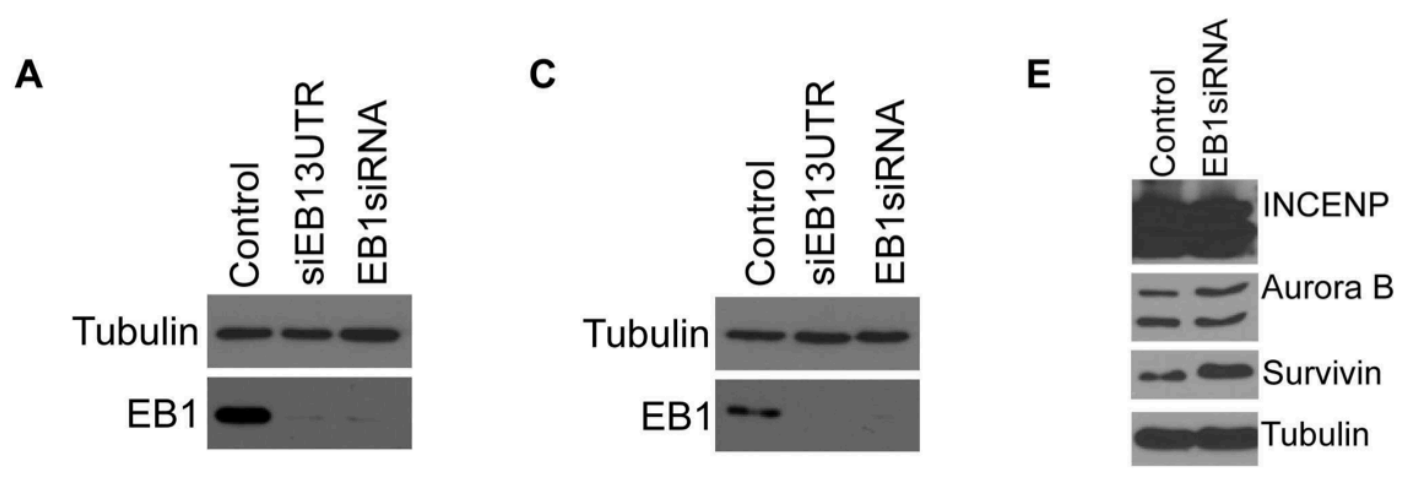

B

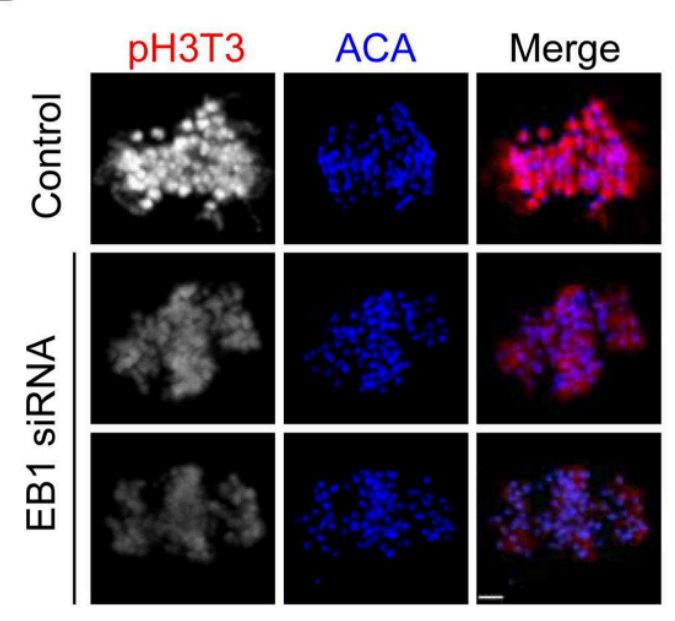

D

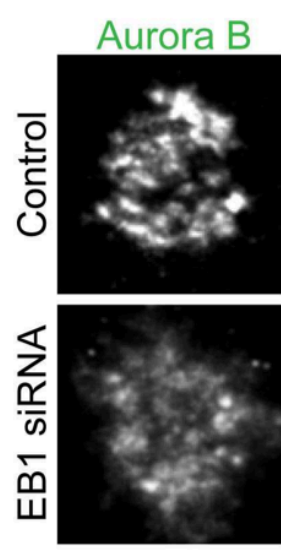

Bub1
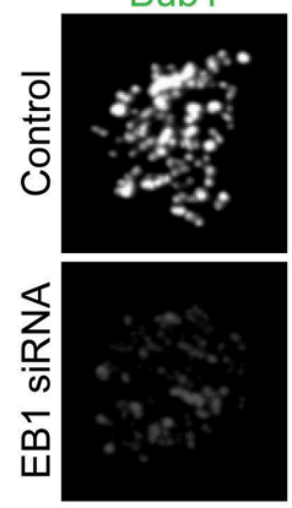

Merge with ACA
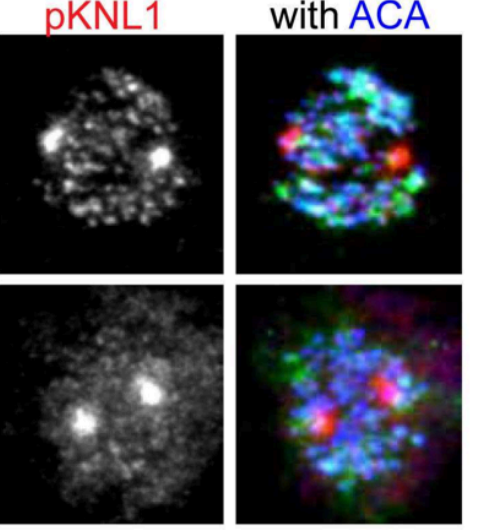

Merge with ACA
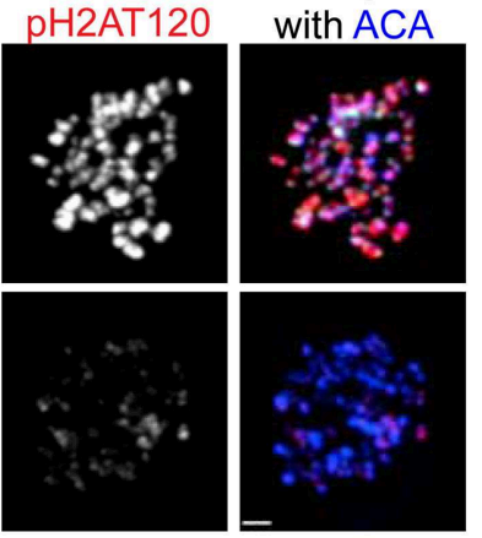

Supplemental Figure 2-1 
EB1 localizes Aurora B to the centromeres in a microtubule dependent manner.

We rescued EB1 depletion phenotypes by multiple methods to ensure that they were not caused by off-target effects. Both the reduction of Aurora B at inner centromeres and the reduced activity at kinetochores were rescued by transfecting a plasmid expressing EB1 mutated to escape siRNA targeting (EB1siRes) (Fig. 2-2 A-E). Moreover, the drop of Aurora B by transfection of a 3'-UTR targeted siRNAs was rescued in a HeLa cell line transfected with or engineered with an integrated copy of EB1-LAP that lacked the 3'UTR (Suppl. Fig. 2-2 A,B). The protein levels of Aurora B and Bub1 were similar to control cell lysates by western blot (Suppl. Fig. 2-2 C).

EB1 is a plus end tracking protein and most of its activities have been associated with microtubules (Morrison et al., 1998). We rescued EB1 depletion with a plasmid expressing a microtubule-binding mutant of EB1 (EB1K89EsiRes) (Hayashi and Ikura, 2003) that was similarly modified to escape siRNA targeting to determine if EB1 used a non microtubule associated activity to localize the CPC. The EB1K89E mutant failed to rescue the reduction in Aurora B levels at the centromeres (Fig. 2-2 A,C) and phosphorylation of KNL1 in EB1 depleted cells (Fig. 2-2 B, D). We conclude that EB1 enriches the $\mathrm{CPC}$ at inner centromeres in a microtubule dependent manner. 
Figure 2-2: EB1 localizes Aurora B at centromere in a microtubule dependent manner.

A) Loss of centromeric Aurora B is rescued by expressing siRNA resistant EB1 (EB1siRes) but not EB1K89E mutant (EB1K89EsiRes). Bar-2.2 $\mu \mathrm{m}$. B) Loss of KNL1(Ser60) phosphorylation was rescued by expressing siRNA resistant EB1 but not EB1-K89E mutant. Bar-2.3um. C) Quantification of immunostaining Aurora B intensities in $\mathrm{A} . * \mathrm{p}$-value $=3.78 \mathrm{e}^{-69} . * * \mathrm{p}$-value $=2.54 \mathrm{e}^{-75} \cdot * * * \mathrm{p}$-value $\left.=1.33 \mathrm{e}^{-35} . \mathbf{D}\right)$ Quantification of immunostaining phospho(S60) - KNL1 intensities in B. * p-value $=$ $5.23 \mathrm{e}^{-42}$. ** $\mathrm{p}$-value $=1.34 \mathrm{e}^{-42}$. *** $\mathrm{p}$-value $\left.=1.02 \mathrm{e}^{-39} . \mathbf{E}\right)$ Western blot of HeLa lysates showing endogenous and GFP-tagged EB1 and KNL1 levels. (Additional rescue experiments are in Fig. S2 A-C) 

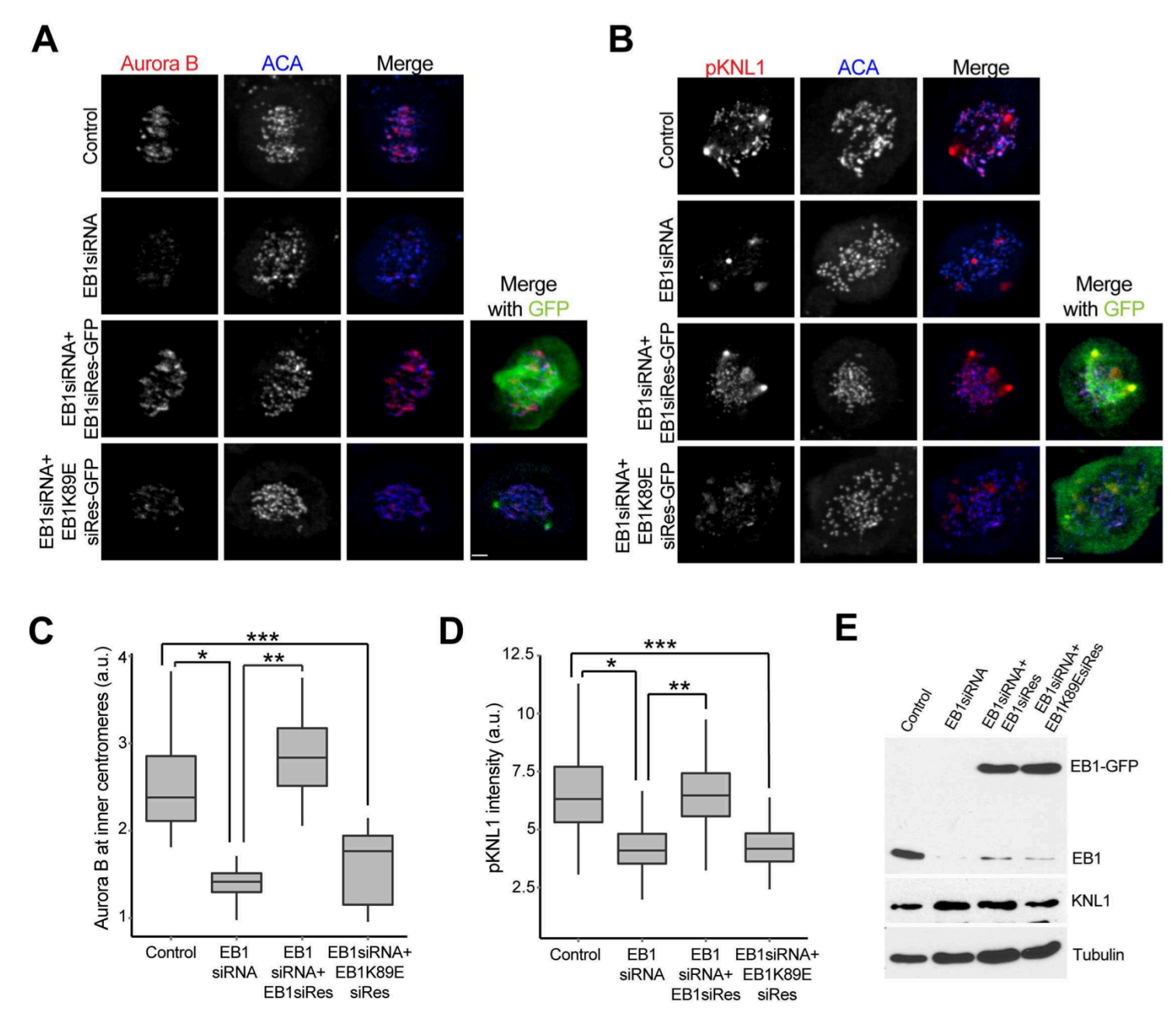

Figure 2-2 
Supplemental Figure 2-2: Additional approaches to rescue EB1 depletion effects on centromeric Aurora $B$ and compare it to microtubule perturbation.

A) HeLa cells were treated with siEB13UTR for 48 hours. Centromeric Aurora B levels were rescued by transfecting pDLAP-EB1 24 hours after siRNA transfection (siEB13UTR+EB1-WT). siRNA resistant EB1-GFP expression is shown in green along with Aurora B (blue) and phospho-KNL1 (red) staining. An EB1-LAP expressing stable line also showed no reduction in inner centromeric Aurora $B$ after transfecting siEB13UTR siRNAs for 48 hours (EB1-LAP+siEB13UTR). Scale bar is $1.6 \mu \mathrm{m}$. B) Quantification of immunostaining intensities in A. ${ }^{*} \mathrm{p}$-value $=3.16 \mathrm{e}^{-102}, * * \mathrm{p}$-value $=$ $2.55 \mathrm{e}^{-116}$. C) Immunoblots to measure protein levels for A. Tubulin staining is shown as a loading control. * indicates a nonspecific band recognized by the anti-Aurora $\mathrm{B}$ antibody (Bethyl). D) Immunno-staining of INCENP and Borealin showing effects of EB1 depletion. Bar-2.1 $\mu \mathrm{m}$. E) HeLa cells treated with EB1siRNA or $3.3 \mu \mathrm{M}$ nocodazole separately or in combination show similar loss in inner centromeric Borealin. F) HeLa cells treated with EB1siRNA or $3.3 \mu \mathrm{M}$ nocodazole separately or in combination show similar loss in inner centromeric INCENP. 
A
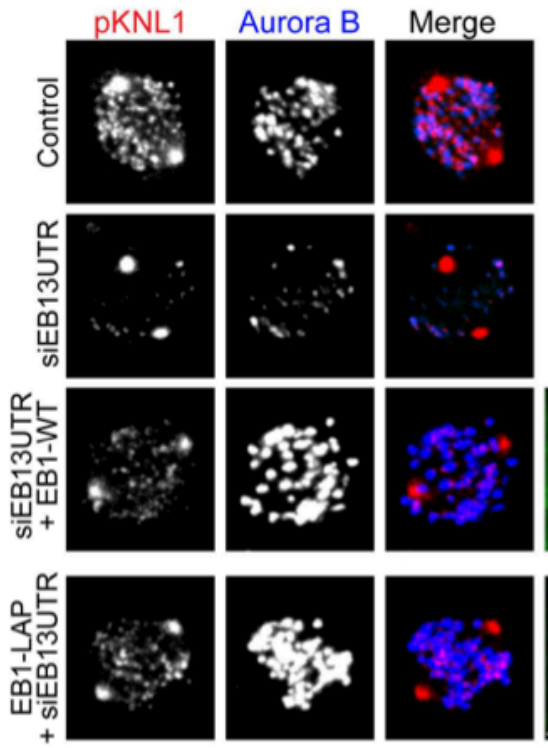

C

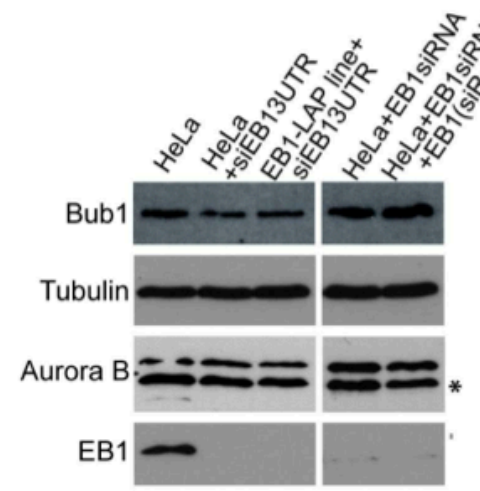

D

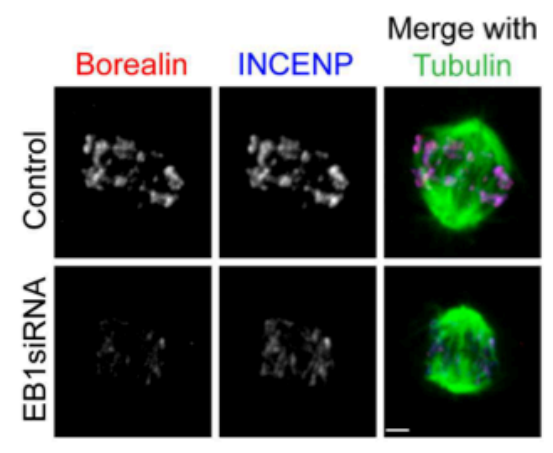

B

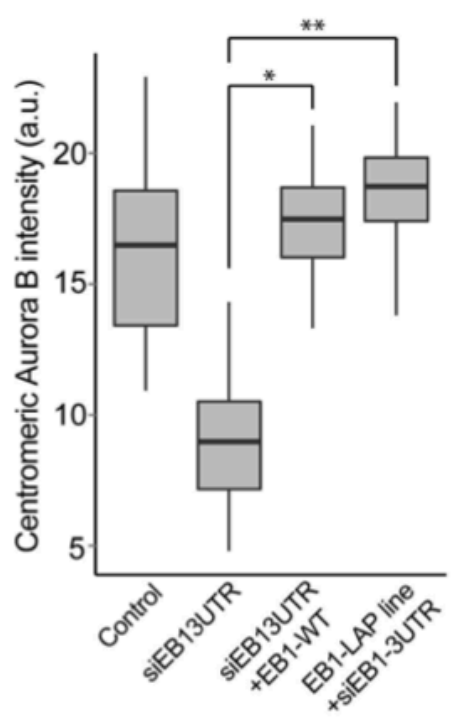

E
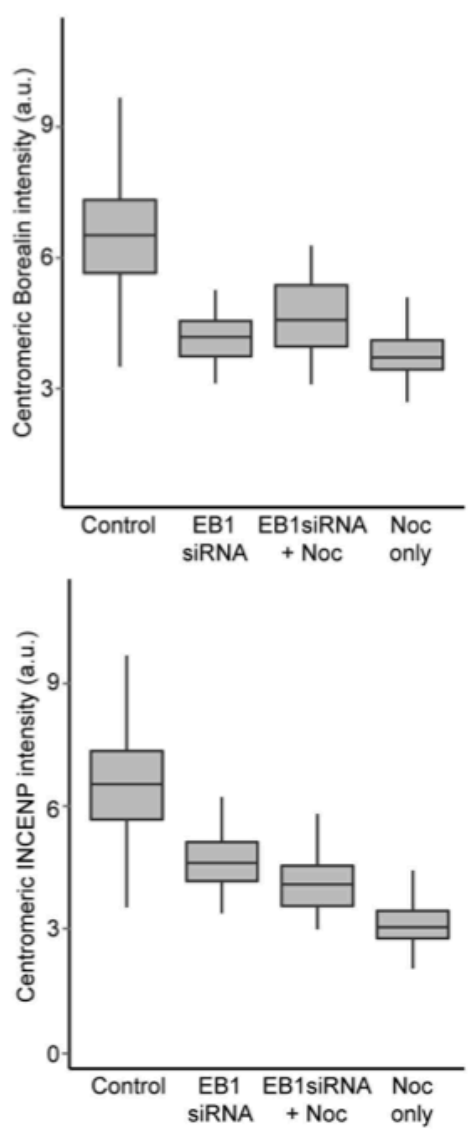

Supplemental Figure 2-2 


\section{Microtubules stimulate the recruitment of Aurora B to inner centromeres}

We decided to reexamine the effects of depolymerizing microtubules on CPC localization using the drug nocodazole. It is important to mention here that treating $\mathrm{HeLa}$ cells with different concentrations of nocodazole has radically different effects on the state of microtubules at the kinetochores. $0.33 \mu \mathrm{M}$ nocodazole has been traditionally used to generate the spindle checkpoint arrest (Hauf et al., 2003) but at this concentration most kinetochores have microtubule foci surrounding them (Fig. 2-3 A) (Brito et al., 2008; Matson et al., 2012). These microtubules are absent in $3.3 \mu \mathrm{M}$ nocodazole (Fig. $3 \mathrm{~A}$ ) (Brito et al., 2008; Matson et al., 2012).

We measured Aurora B levels at centromeres in HeLa cells treated with either $0.33 \mu \mathrm{M}$ or $3.3 \mu \mathrm{M}$ nocodazole for 7 hours. After treatment with $0.33 \mu \mathrm{M}$ nocodazole the amount of Aurora B at the centromeres was similar to DMSO treated controls, while cells treated with $3.3 \mu \mathrm{M}$ nocodazole had significantly reduced levels of Aurora B (Fig. 2-3 A, B and Suppl. Fig. 2-3 A). This suggests that the microtubule foci that surround kinetochores in $0.33 \mu \mathrm{M}$ nocodazole are sufficient to recruit additional Aurora B.

We hypothesized that EB1 and microtubules are in the same pathway. We quantified INCENP and Borealin levels in HeLa cells that were EB1 depleted, treated with $3.3 \mu \mathrm{M}$ nocodazole or had both treatments (Suppl. Fig. 2-2 E,F). The effects of EB1 depletion and microtubule depolymerization were not additive and cells that received both treatments had similar reduction of centromeric CPC as individual treatments. Together these data suggests that EB1 and microtubules are part of the same pathway that enriches centromeric CPC. 
Microtubules cooperate with the histone phosphorylation pathways to recruit Aurora B to inner centromeres

There is a positive feedback loop by which Aurora B targets haspin kinase, to target the CPC (Wang et al., 2011b). Moreover, Aurora B regulates MPS1, which is an activator of Bub1, suggesting a second positive feedback loop where Aurora B localizes Sgo1 to localize the CPC (van der Waal et al., 2012; Saurin et al., 2011). We designed an assay to compare the relative contributions of microtubules and histone phosphorylation in regulating centromeric Aurora B levels. We used 5-iodotubercidin, a small molecule inhibitor to haspin kinase (Wang et al., 2012; De Antoni et al., 2012), referred henceforth as haspin inhibitor (HI) and Reversine, a Mps1 inhibitor that inhibits Bub1 recruitment to kinetochores (van der Waal et al., 2012; Santaguida et al., 2011), to reduce the phosphohistone marks required to localize Aurora B to centromeres. HeLa cells were treated with Nocodazole, Reversine or HI separately or in combination for 30 minutes. All cells were also treated with MG132 to prevent mitotic exit. Both Reversine and HI treatment reduced the histone phosphorylation of its associated pathway to levels below the level of detection (Suppl. Fig. 2-3 B,C). Individual drug treatments caused a severe drop in Aurora B levels (Fig. 2-3 D). There was no additional effect of combining the kinase inhibitors together (R+HI) (Fig. 2-3 D). This suggests that both Haspin and MPS1 are in the same pathway or the pathways have a common component, which we suggest is Aurora B. Adding nocodazole along with reversine and $\mathrm{HI}(\mathrm{R}+\mathrm{HI}+\mathrm{Noc})$ resulted in a significant drop in Aurora B levels compared to R+HI (Fig. 2-3 C,D) $\left(p\right.$-value $=6.6 \mathrm{e}^{-127}$ ). We conclude that microtubules can recruit Aurora B to inner centromeres independent of 
the histone phosphorylation pathways, but the histone phosphorylation pathways are required to obtain the full enrichment of centromeric Aurora B.

These data suggest that EB1 and microtubules are either upstream or work in combination with histone phosphorylation pathways that recruit $\mathrm{CPC}$. To distinguish between these models we asked if targeting Aurora B to centromeres rescued the reduction of the histone phosphorylation pathways observed after EB1 depletion. U2OS cells expressing the DNA targeting domain of $\mathrm{CENPB}^{1-158}$ fused to the INCENP $^{47-920}$ fragment that is under inducible expression by a doxycycline promoter (Liu et al., 2009) were depleted of EB1. CENPB binds alpha satellite DNA sequences at the centromere so that Aurora B is targeted independent of the normal pathways. This clone also lacks the sequences on INCENP required to bind Survivin and Borealin. EB1 depletion in U2OS cells also reduced phospho-KNL1, Bub1 at kinetochores and phospho-Histone H3Thr3 levels when the CENPB-INCENP was not expressed. However, activation of CENPBINCENP expression by doxycycline treatment for 10 hours rescued both Bub1 and phospho-KNL1 levels at the kinetochore in EB1 depleted cells (Fig. 2-3 E-G). We also observed recovery of pH3T3 levels on CENPB-INCENP expression (Fig. 2-3 E). We conclude that EB1/ microtubules dependent enrichment of Aurora B acts upstream of the positive feedback loops that trigger both Histone $\mathrm{H} 2 \mathrm{~A}$ and $\mathrm{H} 3$ phosphorylation pathways that localize Aurora B. 
Figure 2-3: EB1/microtubules act upstream of canonical CPC localization pathways

A) HeLa cells treated with $0.33 \mu \mathrm{M}$ and $3.3 \mu \mathrm{M}$ nocodazole for 7 hours were fixed and stained with Tubulin and Aurora B antibodies. Inset is a projection of $6 \mathrm{Z}$-sections. White arrowheads in $3.3 \mu \mathrm{M}$ nocodazole (tubulin panel) point to centrosomes. Bar-1.8 $\mu \mathrm{m}$. Settings that allow visualization of spindle microtubules obscure the microtubule foci in $0.33 \mu \mathrm{M}$ nocodazole so we have not shown control cells treated with DMSO. B) Average inner centromeric Aurora B levels measured in HeLa cells after the indicated treatments. Box and whisker plot of centromeric Aurora B levels from the same experiment shown in Suppl. Fig. 2-3 A. * p-value $=0.01182$. C) Hela cells were treated with $10 \mu \mathrm{M}$ reversine (R) and $1 \mu \mathrm{M} 5$-iodotubericidine (HI) separately (R only and HI only shown in Suppl. Fig. 2-3 B,C) or in combination $(\mathrm{R}+\mathrm{HI})$ in the presence of MG132. To determine if microtubules can recruit Aurora B in the absence of phospho-histone marks HeLa cells were treated with or without $3.3 \mu \mathrm{M}$ nocodazole along with the reversine, HI and MG132 $(\mathrm{R}+\mathrm{HI}+\mathrm{Noc})$. Control cells were treated with MG132 only. Cells were fixed after 30 minutes and stained with pH3T3, anti-tubulin and anti-Aurora B antibodies. DIC image $(\mathrm{R}+\mathrm{HI}+\mathrm{Noc})$ treatment to show that there is a cell that lacks detectable staining. Bar$1.7 \mu \mathrm{m}$. D) Centromeric Aurora B levels were measured at indicated treatment conditions. In this experiment $1 \mu \mathrm{M}$ reversine $(\mathrm{R})$ was used. ${ }^{*} \mathrm{p}$-value $=6.65 \mathrm{e}^{-127} . \mathbf{E}$ ) Expression of CENPB-INCENP fusion protein in U2OS cells rescued the reduction of Bub1, phosphoKNL1 and pH3T3 levels after EB1 depletion. A stable U2OS line was either mock treated (control) or EB1 siRNA treated with or without CENPB-INCENP induction. Bar$1.8 \mu \mathrm{m}$. F) Quantification of Bub1 levels. * $\mathrm{p}$-value $=1.05 \mathrm{e}^{-262}$. G) Quantification of phospho-KNL1(Ser60) levels. ${ }^{*}$ - - value $=8.95 \mathrm{e}^{-31}$. 
A

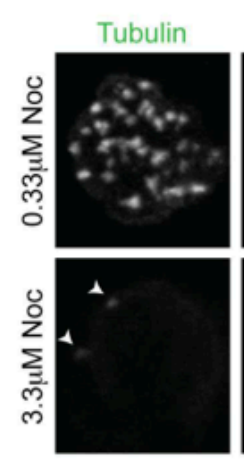

C

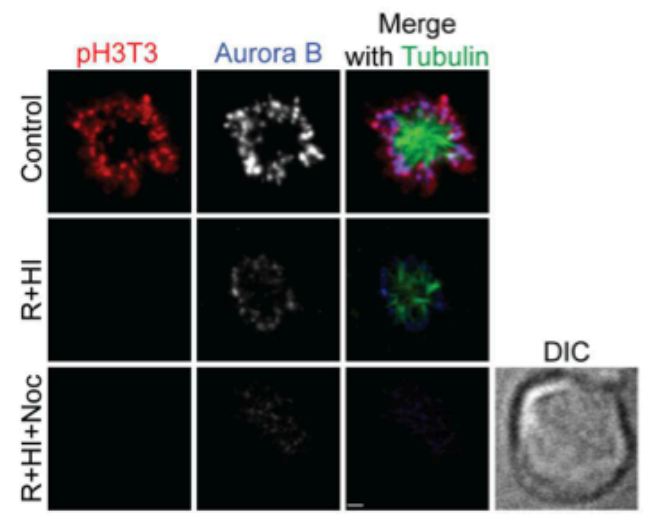

B

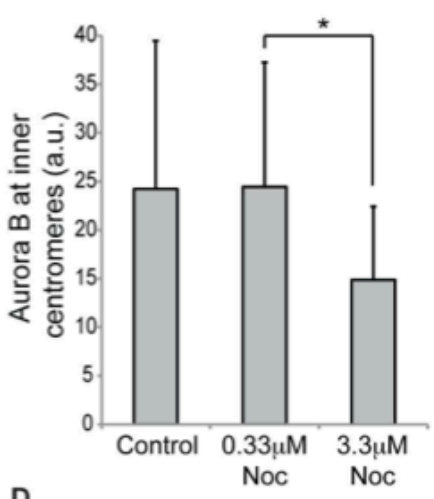

D

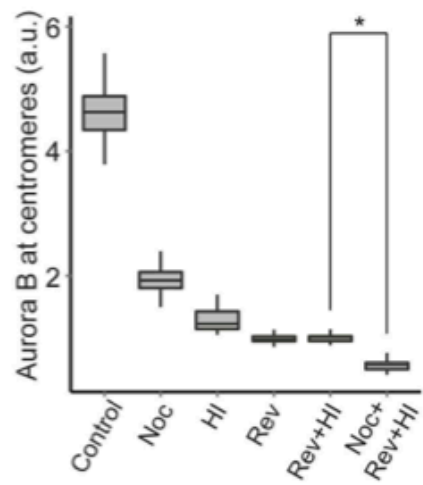

E
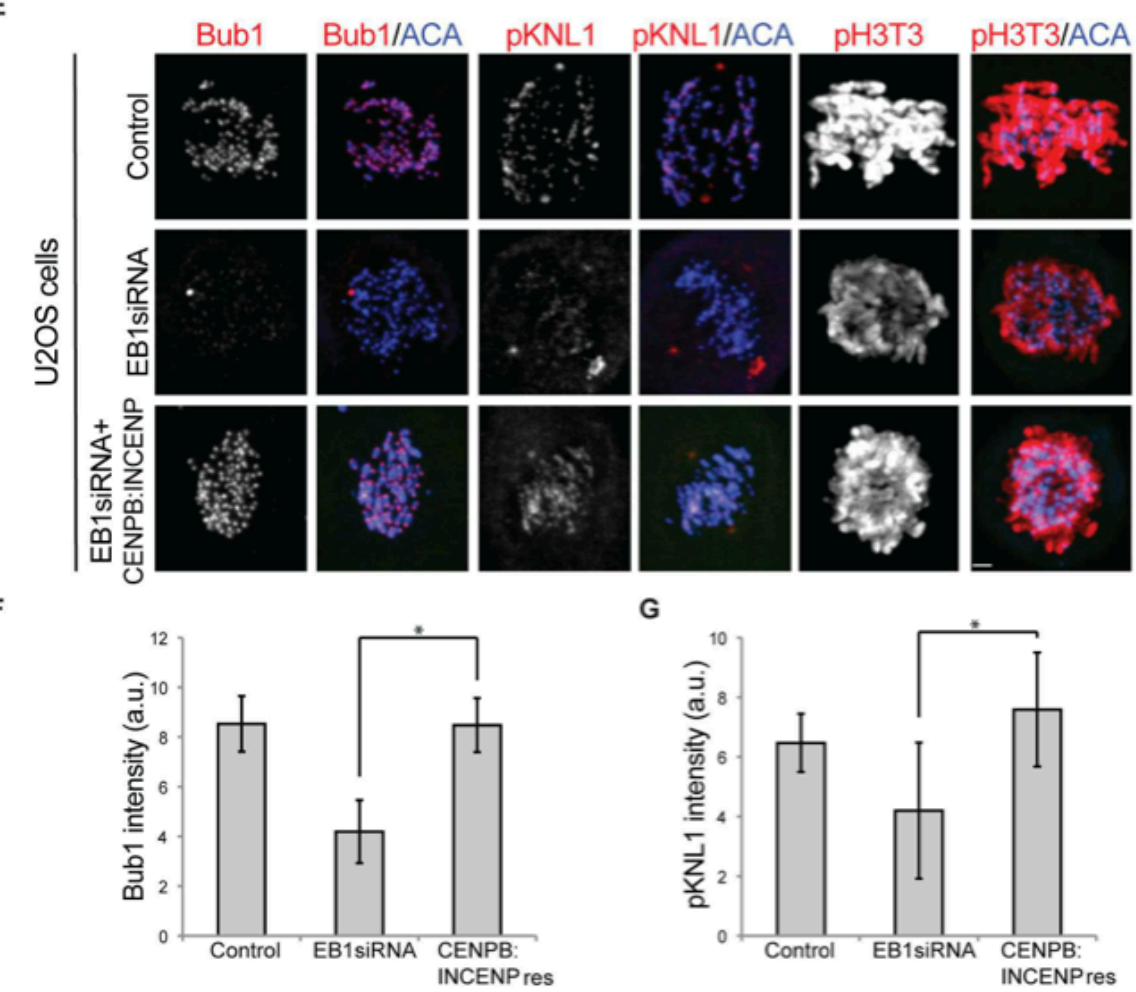

Figure 2-3 
A

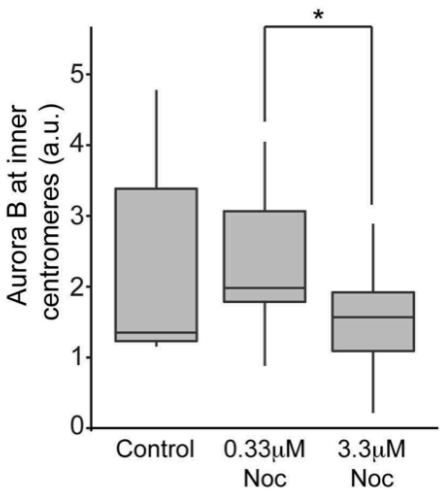

B

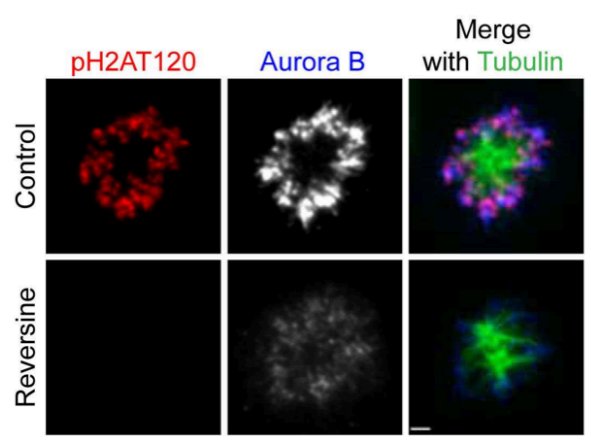

C

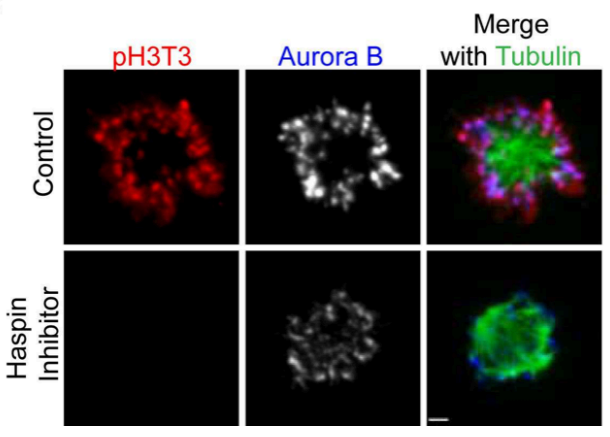

Supplemental Figure 2-3: Centromeric Aurora B is reduced by reversine or 5iodotubericidine (HI) treatment in HeLa cells.

A) Box and whisker plot of centromeric Aurora B levels measured at indicated treatment conditions. ${ }^{*}$ p-value $=0.0118$.

HeLa cells were treated with $10 \mu \mathrm{M}$ reversine (B) or $1 \mu \mathrm{M}$ 5-iodotubericidine (Haspin Inhibitor) (C) along with MG132 for 30 minutes. Cells were then fixed and stained with phospho-Histone H2AThr120, anti-tubulin and anti-Aurora B antibodies. Bar-1.7 $\mu \mathrm{m}$. 
EB1 interacts with Aurora B at the centromeres in prometaphase

Recombinant Xenopus Aurora B bound to a C-terminal fragment of Xenopus INCENP $\left(\mathrm{AI}^{790-856}\right)$ that includes the IN-box purified from E.coli has a basal amount of activity (Sessa et al., 2005). The addition of microtubules stimulated Aurora kinase activity in vitro between 4-6 fold on a myelin basic protein substrate over a range of kinase concentrations (Fig. 2-4 A). However the addition of EB1 did not further stimulate kinase activity in the presence or absence of microtubules (Fig. 2-4 B). We conclude that microtubules can stimulate active kinase in vitro.

EB1 and Aurora B can co-immunoprecipitate in HeLa cells (Sun et al., 2008). To confirm that Aurora B can directly interact with EB1 we purified full length Xenopus EB1 and Xenopus Aurora B bound to a C-terminal fragment of Xenopus INCENP (AI ${ }^{790-}$ ${ }^{856}$ ) that includes the IN-box from E.coli (Sessa et al.). $\mathrm{AI}^{(790-856)}$ bound to beads could pull down EB1 in a concentration dependent manner (Fig. 2-4 C).

To identify the sub-cellular location of the interaction between EB1 and Aurora B we performed Proximity Ligation in situ Assay (PLA) using anti-EB1 and anti-Aurora B antibodies. PLA is an antibody-based technique that allows the visualization of two proteins only if they are in close proximity ( $\sim 35 \mathrm{~nm}$ maximum) with high sensitivity (Söderberg et al., 2006). Standard immunofluorescence staining with anti-EB1 and antiBorealin (CPC member) antibodies showed spindle and inner centromere localization as previously shown (Fig. 2-4 D, Suppl. Fig. 2-4 A). However, most EB1-Aurora B interactions identified by PLA were only found near inner centromeres in prometaphase cells (Fig. 2-4 E,F). This was confirmed by co-staining for Borealin in these cells (prometaphase inset, which shows a stack of 4 Z-sections - Fig. 2-4 E). Similarly, EB1- 
Aurora B interactions were adjacent to Borealin in metaphase-aligned chromosomes (metaphase inset, which was a stack of 7 Z-sections - Fig. 2-4 E). Consistent with the fact that there are no microtubules within the prophase nuclei, we don't see any EB1Aurora B interactions in prophase cells (Suppl. Fig. 2-4 B). To verify the specificity of the interaction we performed PLA reactions lacking either the EB1 or the Aurora B primary antibodies and very little PLA signal was produced (Suppl. Fig. 2-4 C, C'). We conclude that subsets of EB1 and Aurora B interact near or at inner centromeres, which is consistent with the regulation of Aurora B localization by EB1. 
Figure 2-4: EB1 is in close proximity to Aurora B at centromeres.

A) Microtubules stimulate Aurora B bound to $\operatorname{INCENP}^{790-856}\left(\mathrm{AI}^{790-856}\right)$ activity on Myelin Basic protein in vitro. Assay was performed with or without $3 \mu \mathrm{M}$ taxol-stabilized microtubules (MT). B) EB1 does not stimulate Aurora B kinase activity alone or in combination with microtubules. $\mathrm{AI}^{790-856}$ in vitro kinase assay, using MBP as substrate, showing effect of adding $3 \mu \mathrm{M}$ taxol-stabilized microtubules (MT) and EB1 (100ng) separately or in combination. Kinase activity of $20 \mathrm{nM} \mathrm{AI}^{790-856}$ was assayed similarly as in A. C) Direct interaction between EB1 and the catalytic subunit of the CPC. Recombinant Xenopus Aurora B (N-terminal GST tag) bound to a fragment of INCENP $^{790-856}$ (GST-AI) was bound to Glutathione Sepharose-4 beads, incubated with the indicated concentration of recombinant EB1, the beads were washed and eluted with Glutathione and the two peak fraction of the elutions (E1, E2) were quantified by immunoblot. Beads bound to GST were used as a control. D) HeLa cells were methanol fixed and stained with antibodies against EB1 and Borealin. Images shown are merge of images shown in Suppl. Fig. 2-4 A. Bar-2.6um. E) HeLa cells processed to image Borealin and Tubulin by immunofluorescence and the close proximity of EB1 and Aurora $\mathrm{B}$ by PLA. Insets illustrate PLA at individual centromeres. Prometaphase inset is a projection of $4 \mathrm{Z}$-sections, and the metaphase inset is a projection of $7 \mathrm{Z}$-sections. Bar2.4 $\mu \mathrm{m}$. (PLA controls are in Suppl. Fig. 2-4 C-C'). F) Quantification showing percentage occurrence of PLA spots adjacent to centromeres from the PLA experiment shown in C. 
A

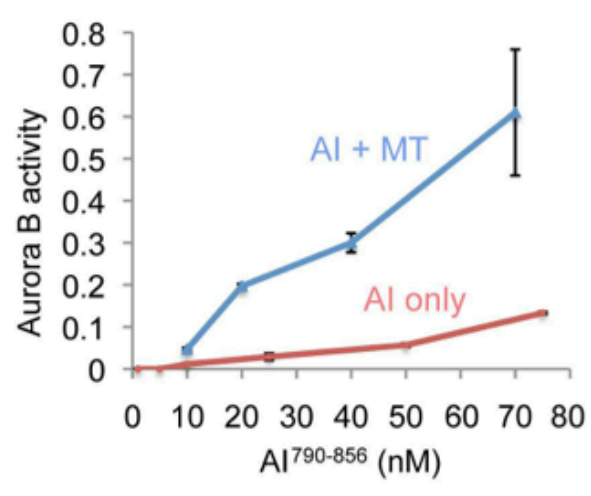

C

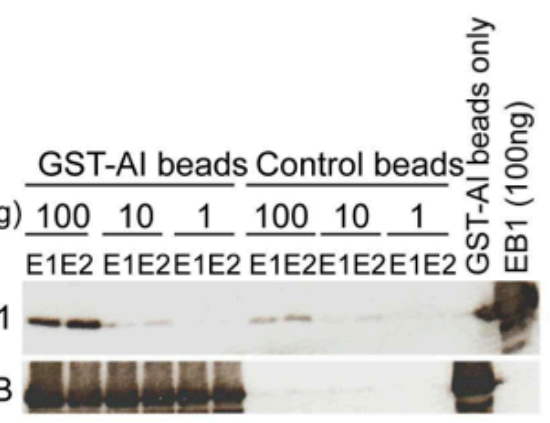

E

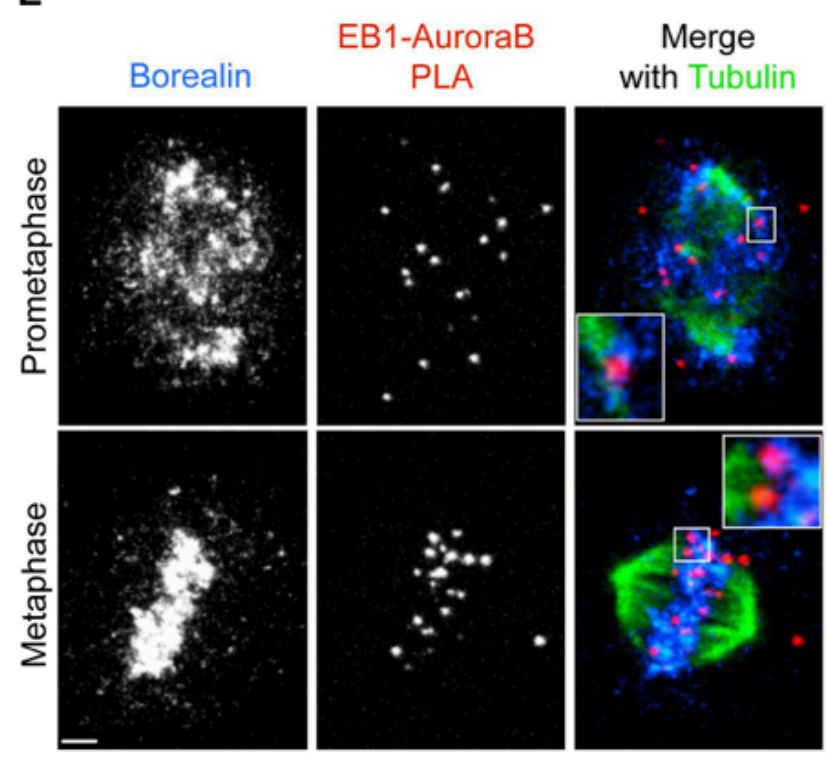

B
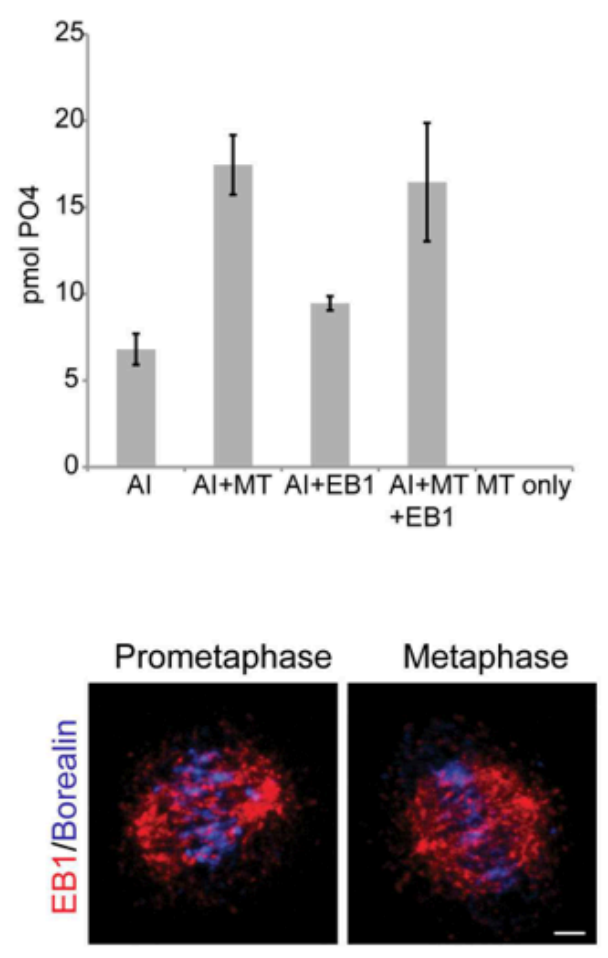

F

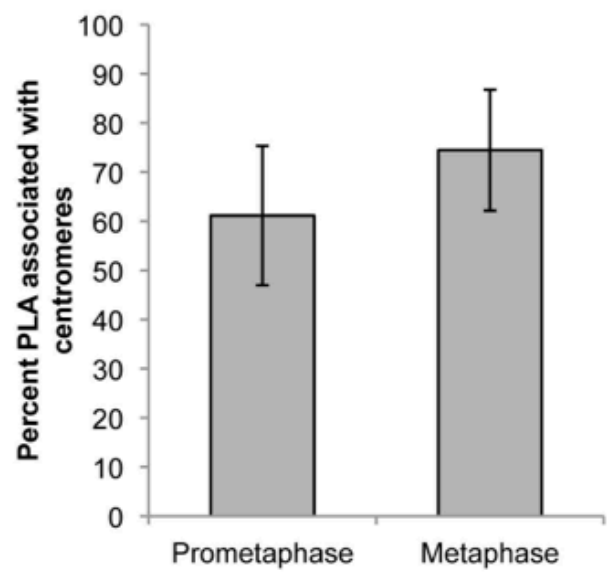

Figure 2-4 
A

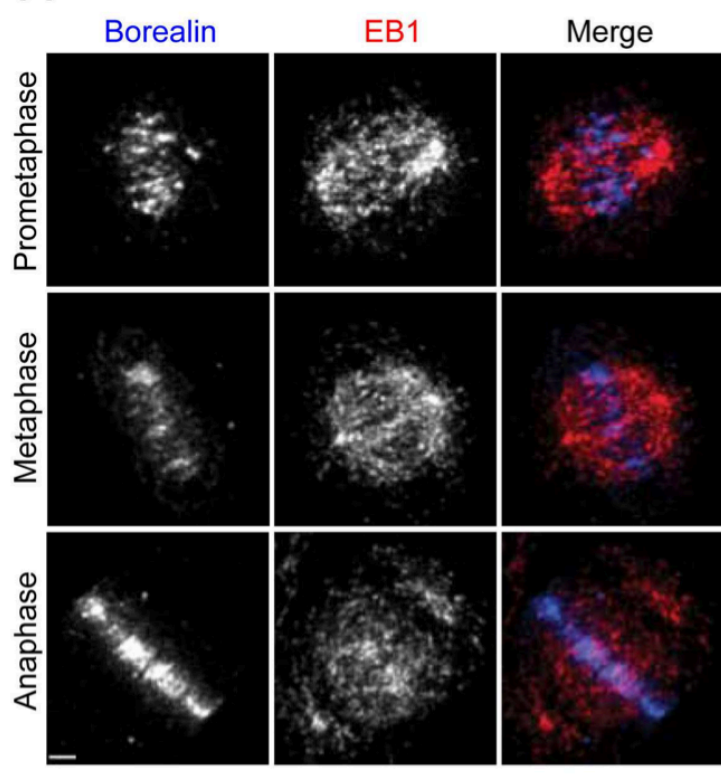

B

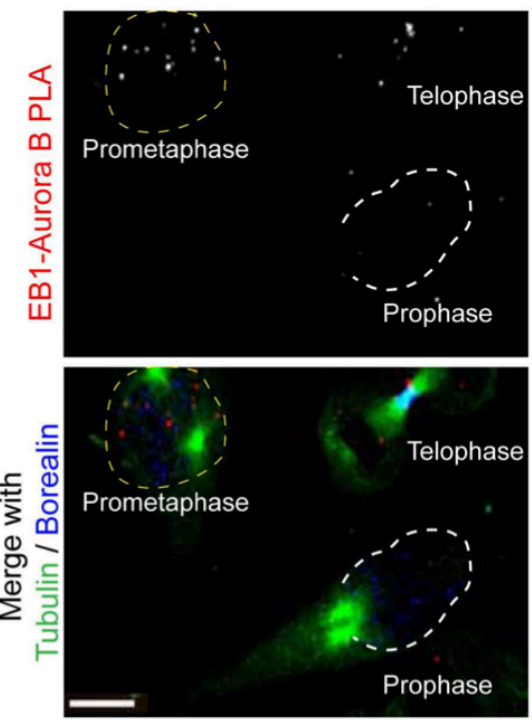

C

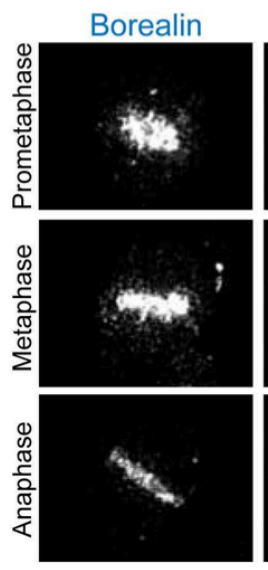

Merge

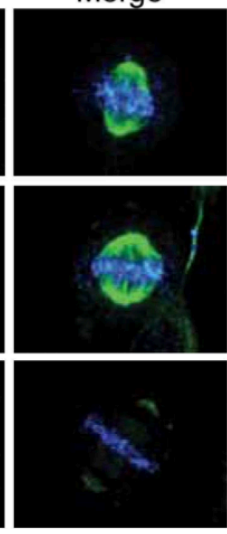

C'

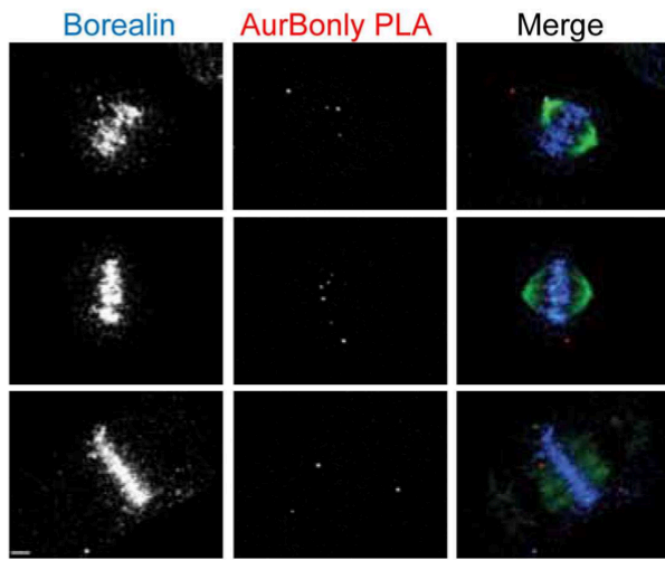

Supplemental Figure 2-4: The bulk of EB1 and CPC localize to distinct sub-cellular domains but interact near the kinetochores in prometaphase.

A) Single channel split of images shown in Fig. 2-4 D. Bar-2.6um. B) EB1-Aurora B PLA signals are absent in prophase cells. A representative prophase nucleus is outlined in white dashed line. A prometaphase cell with numerous PLA dots is highlighted by yellow dashed line in the same image. Bar $=8 \mu \mathrm{m}$. C) and C') PLA controls for Fig. 2-4 E. Bar$2.8 \mu \mathrm{m}$, same for both $\mathrm{C}$ and $\mathrm{C}^{\prime}$. 
Aurora B interacts with K-fibers, PreK-fibers and astral microtubules in mitosis

PLA was performed to identify the sub-cellular locations where Aurora B was in close proximity to tubulin. We utilized Xenopus S3 cells for two reasons. First, these cells possess a normal karyotype and remain very flat in mitosis to provide outstanding imaging. Second our Xenopus Aurora B antibodies are highly specific and provide very reproducible signals in the PLA assay. We performed PLA for Aurora B-tubulin and then the cells were further processed by standard immunofluorescence with antibodies directly conjugated with fluorophores to tubulin and INCENP to generate fiducial marks on the spindle. The PLA signal in prometaphase cells could be detected at inner centromeres (Fig. 2-5 A, inset yellow box) and adjacent to inner centromeres on microtubules (Fig. 25 A, inset orange box). This is similar to the EB1-Aurora B interactions seen by PLA and is consistent with our observation that EB1/microtubules localizes Aurora B to inner centromeres. In addition, we detected additional PLA signal throughout the spindle. Cells were subjected to a brief ice treatment to destabilize non-kinetochore associated microtubules (Fig. 2-5 A ice). We observed a significant drop in PLA signal in ice treated prometaphase cells, including most kinetochores that were not situated near centrosomes (Fig. 2-5 A prometaphase). Some kinetochore/centromere PLA signals persisted in metaphase suggesting that Aurora B can interact with K-fibers. However, most of the metaphase signals throughout the spindle were cold sensitive suggesting that Aurora B can interact with astral microtubules. To verify the specificity of the PLA signals, we performed parallel assays where the anti-Aurora B antibody was omitted and the PLA signal was greatly reduced (Suppl. Fig. 2-5 A). 
We hypothesized that the interactions between EB1-Aurora B and TubulinAurora B that we visualize at prometaphase kinetochores were with PreK-fibers. This hypothesis is strongly supported by the inner centromere enrichment of Aurora B in cells treated with 0.33 uM nocodazole (Fig. 2-3 A). In addition, after cold treatment the bulk of Aurora B tubulin interactions next to prometaphase kinetochores were lost. PreKfibers are small bundles of microtubules that protrude from kinetochores before they make mature attachments and can mediate lateral attachments between dynein on kinetochores and microtubules (Khodjakov et al., 2003). PreK-fibers are difficult to distinguish in a whole spindle but they are readily visualized in monastrol and after cells are washed out of nocodazole.

Xenopus S3 cells were incubated in monastrol and cells were stained for $x l \mathrm{Ndc} 80$ to visualize kinetochores, tubulin to visualize the spindle and PLA to detect where Aurora B was in close proximity to microtubules. Fig. 2-5 B shows 4 Z-sections through a monopolar spindle with centrosomes in the center. Chromosomes tend to have a distinct orientation in monastrol, where the kinetochore facing the pole forms K-fibers with the centrosome, while its sister generates PreK-fibers extending away from the central pole axis (Khodjakov et al., 2003). We find the PreK-fiber microtubule bundles that extend out of kinetochores directed away from the central pole have PLA signals indicating interaction between Aurora B and tubulin (Fig. 2-5 B). The PLA signals on the microtubule bundles pointing away from the center are almost always stronger than those on bundles toward the center, arguing that Aurora B has specificity for PreK-fibers over K-fibers. 
We similarly stained cells that were washed out of nocodazole into fresh media for 5 and 15 minutes. We observed prominent PLA densities between Aurora B and tubulin emanating from most kinetochores generating PreK-fibers 5 minutes after washout (Fig. 2-5 C, 5 minute). Also note that the PLA signals were lost in cells treated with nocodazole (Fig. 2-5 C, 0 minute), confirming that our PLA assay only detects when Aurora B is close to microtubules and not simply free tubulin. After 15 minutes many chromosomes had aligned, however the chromosomes that were not aligned had prominent PreK-fibers (Fig. 2-5 C, 15 minute). The inset shows clear bundles of microtubules next to these kinetochores that have bright Aurora B-tubulin PLA densities. These data suggest that AuroraB binds to PreK-fibers. 
Figure 2-5: Aurora B interacts with distinct classes of spindle microtubules.

A) Xenopus S3 cells were processed to image INCENP and Tubulin by immunofluorescence and the close proximity of Tubulin and Aurora B by PLA. Insets within the merge show single Z-sections of Aurora B-Tubulin interactions at the centromere (yellow boxes) and at the kinetochores (orange boxes). Lower panel shows the effect of ice treatment on S3 cells prior to fixation. Bar-11 $\mu \mathrm{m}$. B) Monastrol treated Xenopus S3 cells were processed to image Hec1 and Tubulin by immunofluorescence and the close proximity of Tubulin and Aurora B by PLA - projection of 4 Z-sections. C) Nocodazole washout experiments were performed to show Aurora B specifically enriched on microtubule bundles near kinetochores, which are PreK-fibers. Xenopus S3 cells were fixed and stained 5 minutes (projection of $7 \mathrm{Z}$-sections) and 15 minutes (projection of 9 Z-sections) after nocodazole washout. Aurora B-tubulin PLA signals are shown with Hec1 and Tubulin co-staining. (Individual Z slices of whole cell projections are shown in Video 1-4) 


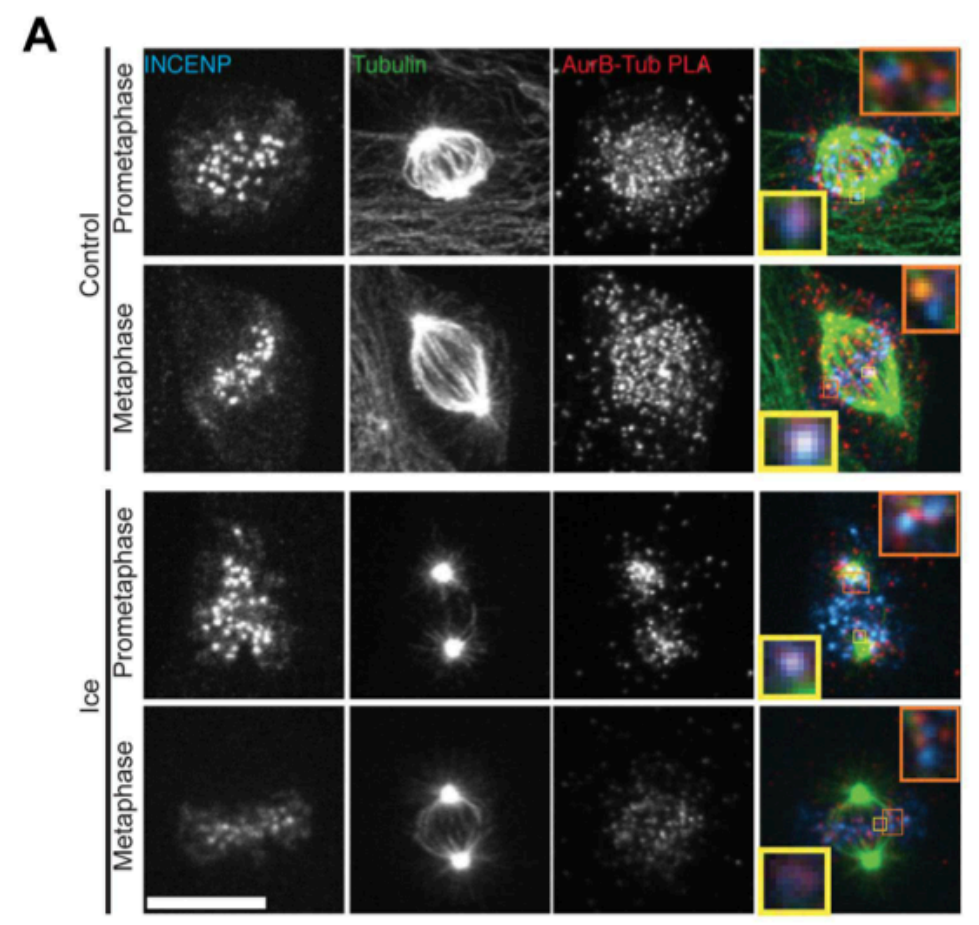

B

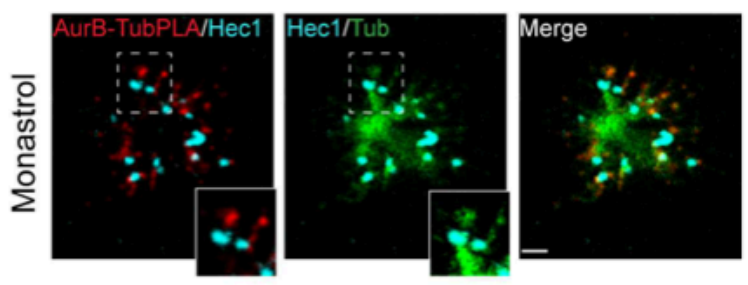

C

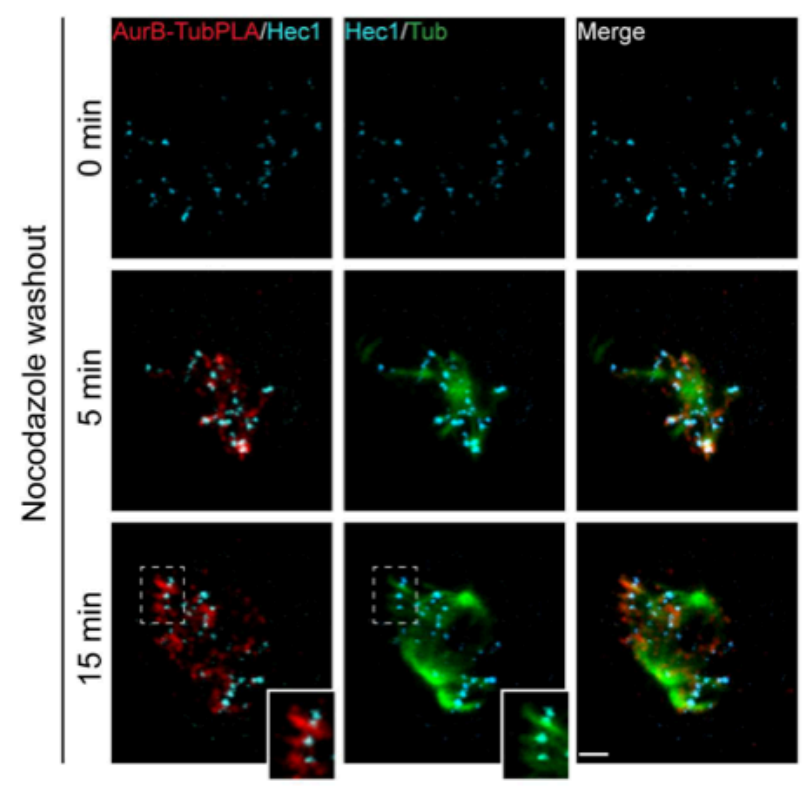

Figure 2-5 
EB1 dependent localization of Aurora B to centromeres in prometaphase is required to phosphorylate kinetochore and chromatin substrates

We postulated that one reason that it has been difficult to measure the effects of microtubules on Aurora B localization and phosphorylation in the past is that Aurora B activity in prophase nuclei is independent of microtubules. Aurora B phosphorylates histone H3 on Serine-10 (pH3S10 in figures) on the arms of mitotic chromosomes in late G2/Prophase (Hendzel et al., 1997) and this phosphorylation persists through early anaphase. Indeed depletion of EB1 had little effect on phospho-Histone H3Ser10 levels (not shown), and we postulate that EB1 is not required for prophase activity of Aurora B, which persists into prometaphase. To separate Aurora B phosphorylation in prophase and prometaphase we applied a recently developed assay that specifically visualizes the generation of Aurora B phosphorylation in prometaphase cells (Wang et al., 2011a).

EB1 depleted cells were treated with a reversible Aurora kinase inhibitor, ZM447439 (ZM) and phosphorylation was measured on Histone H3Ser10 by phosphospecific antibodies after washing out the drug. The small molecule monastrol was used to generate monopolar spindles thereby limiting the variables of tension forces generated by the mitotic spindle and cells were arrested in mitosis by the addition of the proteosome inhibitor MG132 for 1 hour. (Experimental outline shown in Fig. 2-6 A) Cells depleted of EB1 were unable to phosphorylate Histone H3 on Ser10 15 minutes after ZM washout (Fig. 2-6 B, D). Aurora B and phospho-KNL1 levels were similarly affected (Fig. 2-6 B,C,E and F). We conclude that EB1 is required for phosphorylation of both kinetochore and chromatin substrates by Aurora B in prometaphase. 
We modified the ZM-washout assay (Wang et al., 2011a) to determine if spreading Aurora B activity to kinetochores was microtubule-dependent in prometaphase (Experimental outline shown in Suppl. Fig. 2-5 B). Aurora B levels were reduced in HeLa cells after treating them with ZM (1 hour) and $3.3 \mu \mathrm{M}$ nocodazole (10 minutes) (Fig. 2-6 G,H - 0min). Centromeric Aurora B levels increased over time in cells that were shifted to ZM-nocodazole free media (Fig. 2-6 G,H - Recov 10min), while Aurora B levels did not increase after ZM washout in cells held in nocodazole (Fig. 2-6 G,H Recov in Noc 10min). Microtubules were also required for cells to phosphorylate kinetochores after ZM washout as measured by staining with phospho-KNL1 antibodies (Fig. 2-6 G) (Welburn et al., 2010). We conclude that microtubules are required to both localize Aurora B to inner centromeres and for Aurora B dependent phosphorylation of kinetochore substrates in prometaphase. 
Figure 2-6: EB1 and microtubules are required for Aurora $B$ to phosphorylate kinetochores and chromatin substrates in prometaphase

A) Experimental outline of experiment shown in B,C. B) HeLa cells treated with ZM447439, MG132 and Monastrol were washed out of ZM447439 to monitor recovery of phospho-HistoneH3Ser10 (pH3S10) after 15 minutes. EB1 depleted cells are compared with control cells treated similarly and fixed immediately (ZMwashout- 0 min) or replaced in ZM-free media and fixed after 15 minutes (ZMwashout-15min). Bar1.6 $\mu \mathrm{m}$. C) Spreading of Aurora activity from centromeres to kinetochores (pKNL1) requires EB1. Note centrosomal staining is an artifact, and kinetochore staining represents phospho-KNL1. Bar-1.7 $\mu \mathrm{m}$. D) Quantification of average total pH3S10 recovery in B. ${ }^{*}$ p-value $=2.2 \mathrm{e}^{-05}$. E) Quantification of kinetochore phosphorylation in C. F) Centromeric Aurora B levels from B. ${ }^{* *}$ p-value $=5 \cdot 3 \mathrm{e}^{-157}$. (Scheme shown in A) G) Microtubules are required for the recovery of inner centromeric Aurora B and Aurora phosphorylation of kinetochores after removal of ZM. Note that the phospho-KNL1 has non specific staining of centrosomes (Welburn et al., 2010). H) Quantification of inner centromeric Aurora B intensities in $G(n>160)$. * $p$-value $=4.19 \mathrm{e}^{-91}$ (Scheme for experiment shown in Suppl. Fig. 2-5 B) 
A

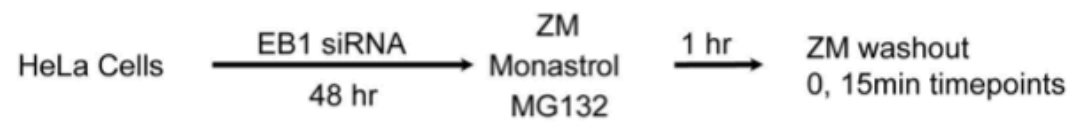

B

C

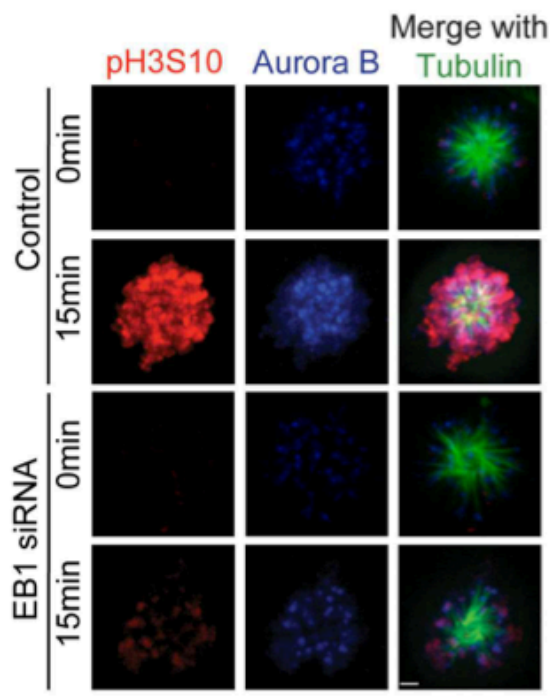

E

D

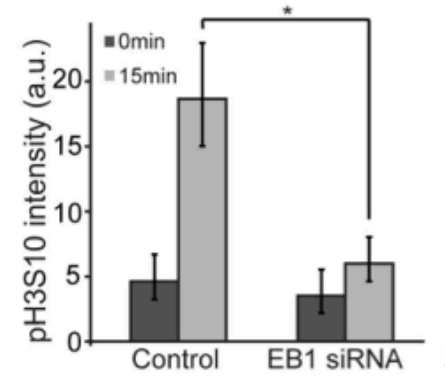

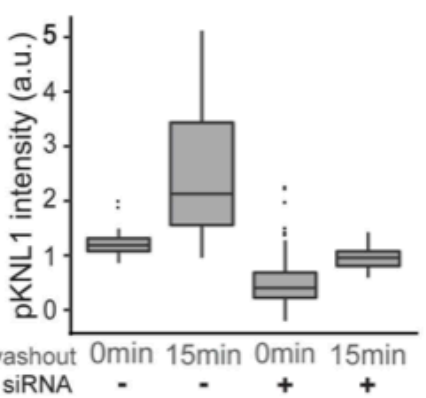

Merge Merge with pKNL1 with ACA Tubulin

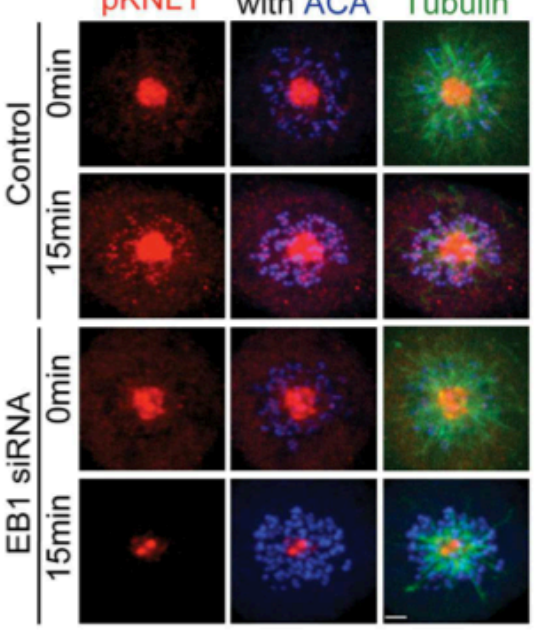

F

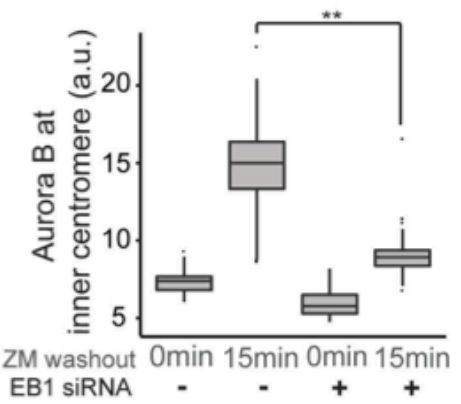

G

Merge

H
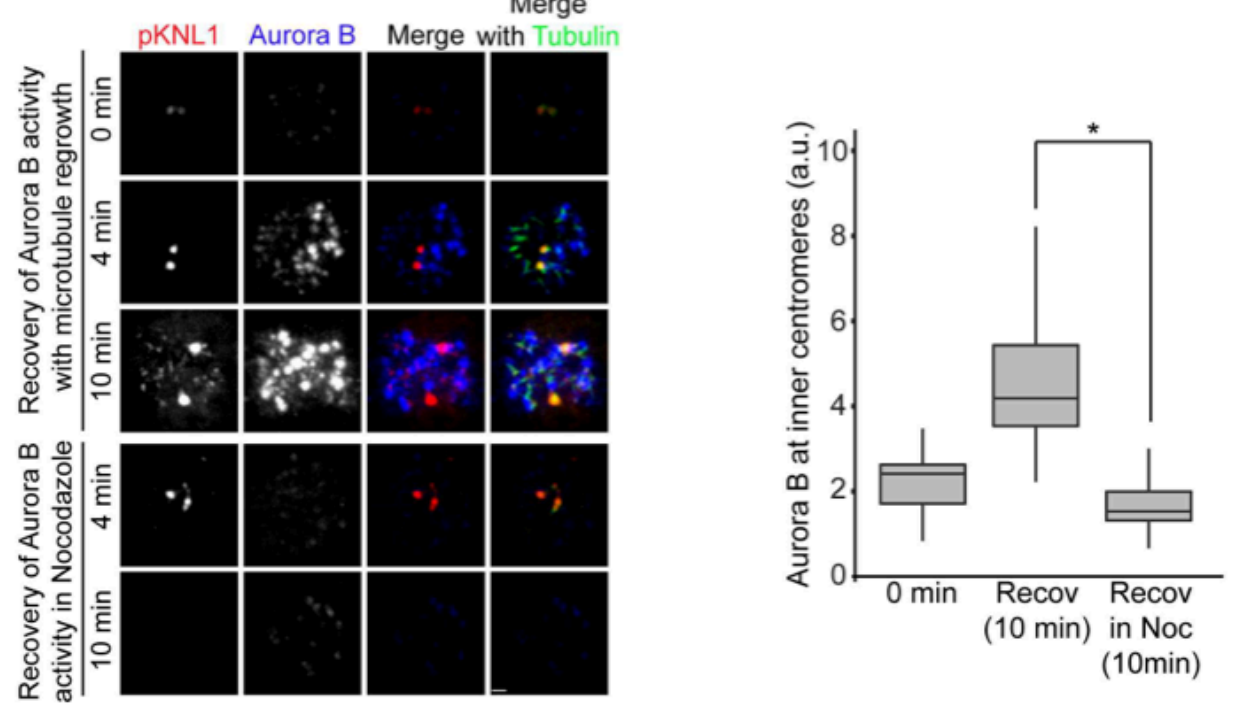

Figure 2-6 


\section{Aurora B activity on Chromatin is regulated by microtubules in prometaphase}

We compared the microtubule dependence of Aurora B activity on noncentromeric chromatin in prophase and prometaphase by washing cells out of ZM in the presence or absence of nocodazole (assay scheme shown in Suppl. Fig. 2-5 B). The presence or absence of microtubules made little to no difference to the prophase cells (Fig. 2-7 A). In contrast, the absence of microtubules decreased the amount of chromatin phosphorylation in prometaphase cells. We conclude that prometaphase, but not prophase, Aurora B activity levels are dependent on microtubules.

We further examined the microtubule stimulation of Aurora kinase activity on prometaphase chromatin. We observed both a spatial and quantitative correlation between the microtubules and Aurora B activity (Fig. 2-7 B). Before washing out the ZM there was weak phosphorylation of phospho-H3S10 and this was spatially restricted to areas of chromatin that were adjacent to microtubule foci that are likely centrosomes (Fig. 2-7 B, 0 min). After washing out both $\mathrm{ZM}$ and nocodazole we observed robust spreading of Ser10 phosphorylation on Histone H3 throughout chromatin over time. Spreading did not happen in the cells that remained in nocodazole (Fig. 2-7 B,C).

Microtubules re-growth was not homogenous and in some cells we could detect microtubules only at centrosomes and in other cells we could detect microtubules at both centrosomes and at foci that are likely PreK-fibers. We plotted the amount of phosphoH3S10 activity in each cell as a function of the amount of polymerized tubulin in the cell. The single cell intensities fell on a diagonal signifying correlation and the $\mathrm{R}^{2}$ value was 0.91 (Fig. 2-7 D). This tight correlation held true for each of the time points (Suppl. Fig. 
2-5 C). This suggests a surprising connection between the microtubules of the mitotic spindle and histone phosphorylation throughout chromatin.

We have shown that a major role of EB1 and microtubules is to localize the CPC to centromeres. To measure the importance of localizing the $\mathrm{CPC}$ to inner centromeres in the ZM washout assay (Wang et al., 2011a), we replaced the endogenous survivin subunit with the survivin H80A mutant that is unable to bind Histone $\mathrm{H} 3$ phosphorylated on Threonine-3 by Haspin (Niedzialkowska et al., 2012). Aurora B kinase was reactivated by removal of ZM and the recovery of phospho-Histone H3Ser10 activity was followed by immunofluorescence over the next 15 minutes (scheme in Fig. 2-8 A). PhosphoHistone H3Ser10 staining was apparent on chromatin 15 minutes after washing out ZM in cells expressing wild type survivin (WT res) (Fig. 2-8 B,C 15min). In contrast cells expressing survivin ${ }^{\mathrm{H} 80 \mathrm{~A}}$ were unable to phosphorylate chromatin (Fig. 2-8 B,C - H80A res). As expected, Aurora B did not localize to centromeres in cells expressing the survivin $^{\mathrm{H} 80 \mathrm{~A}}$ (Fig. 2-8 B). We verified the Aurora B and Survivin protein levels in each condition by western blot (Fig. 2-8 D). We conclude that preventing the localization of the $\mathrm{CPC}$ to inner centromeres phenocopies the loss of spreading of Aurora kinase activity on chromosome arms seen after depletion of either microtubules or EB1. Together our data suggest that $\mathrm{EB} 1 /$ microtubules localize the $\mathrm{CPC}$ to prometaphase centromeres to phosphorylate both kinetochores and chromatin. 
Figure 2-7: Aurora B activity on chromatin is regulated by microtubules in prometaphase, but not in prophase

A) Phospho-Histone H3Ser10 (pH3S10) and tubulin immunostaining of prophase and prometaphase cells at indicated conditions. Insets show chromatin staining. Bar-10um. (Assay scheme shown in Suppl. Fig. 2-5 B) B) HeLa cells in prometaphase immunostained for tubulin, phospho-Histone H3Ser10 (pH3S10) and chromatin at indicated conditions. Bar-2.2 $\mu \mathrm{m}$. C) Relative pH3S10 intensity plotted as a function of time after ZM-washout in presence or absence of nocodazole. D) Correlation shown between total cellular pH3S10 intensity from all time points and total tubulin intensity measured per cell $\left(\mathrm{R}^{2}=0.91\right)$. Cellular $\mathrm{pH} 3 \mathrm{~S} 10$ intensities at 0 minute and recovery phases in absence of nocodazole are represented as blue diamonds and intensities from cells in nocodazole are in red squares. 


\section{A}

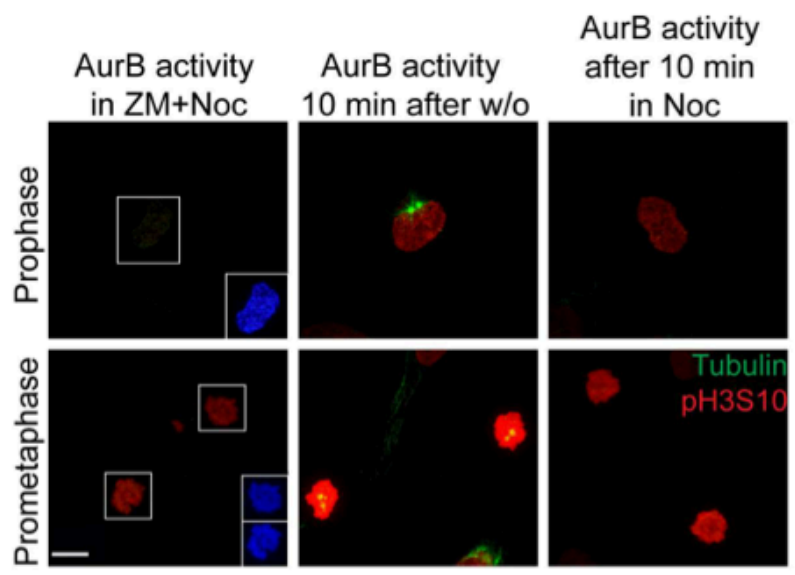

B

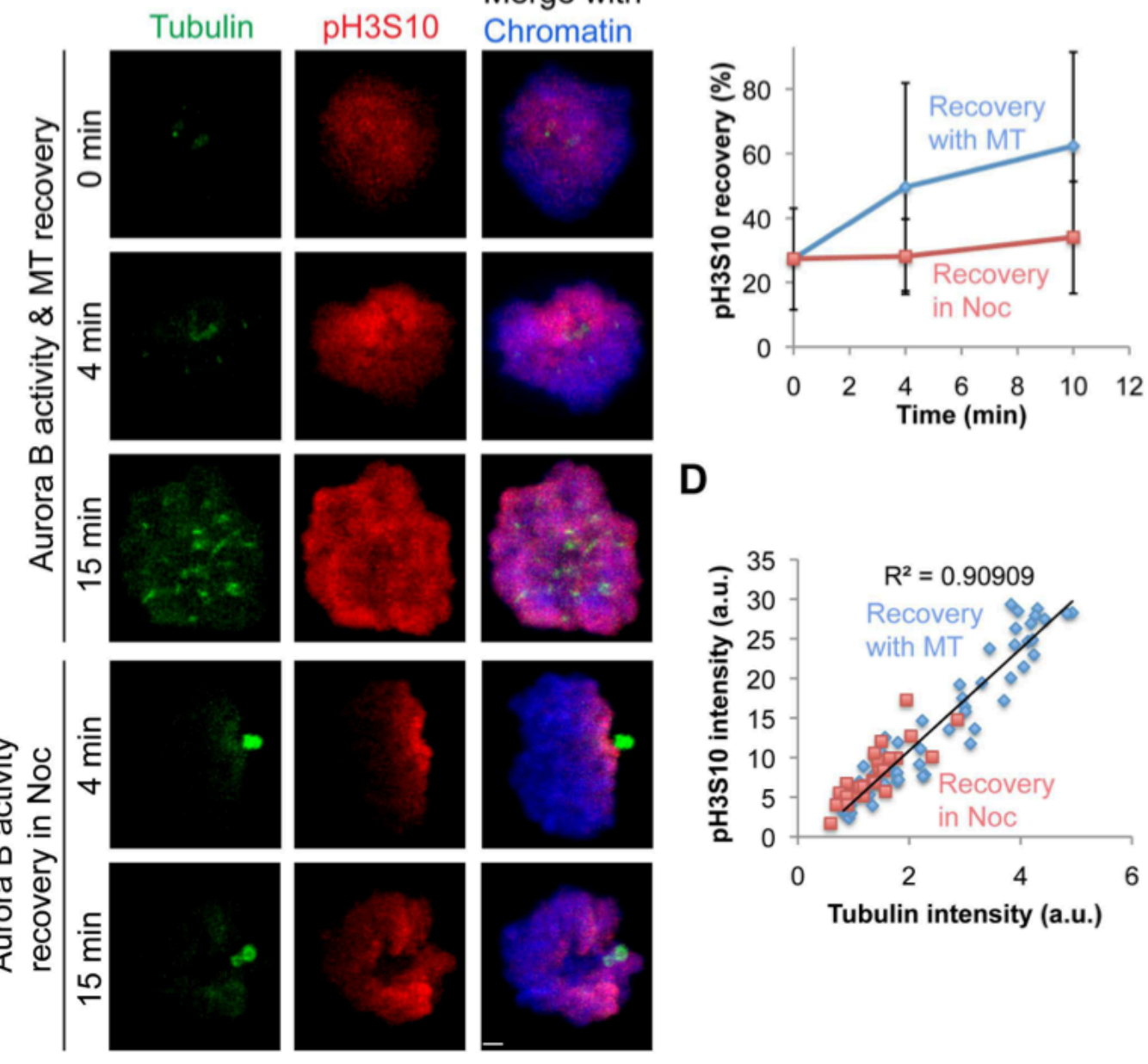

Figure 2-7 
Figure 2-8: CPC activity spreads from centromeres to chromosome arms after nuclear envelope breakdown.

A) Assay scheme. B) Cells that have endogenous survivin replaced with the survivin ${ }^{\mathrm{H} 80 \mathrm{~A}}$ mutant are unable to phosphorylate Histone H3Ser10 in the ZM-washout assay. HeLa cells stably expressing vector only, survivin-myc (siRNA resistant) and survivin ${ }^{\mathrm{H} 80 \mathrm{~A}}$-myc (siRNA resistant) were treated with survivin siRNA for 48 hours followed by 1 hour in ZM447439, MG132 and monastrol. Bar-2.7um. C) Average total phospho-Histone H3Ser10 intensity at 0 and 15 minutes in mock treated (vec. only) and survivin siRNA treated - vec. only (vec. only+siRNA), survivin-myc (WT res) and survivin ${ }^{\mathrm{H} 80 \mathrm{~A}}$-myc (H80A res) expressing cells. D) Western blot showing Aurora B, survivin-myc and endogenous survivin levels. 
A

$$
\underset{\substack{\text { Survivin } \\
\text { stable lines }}}{\stackrel{\text { Survivin siRNA }}{\longrightarrow}} \begin{gathered}
\text { Monastrol } \\
\text { MG132 }
\end{gathered} \stackrel{1 \mathrm{hr}}{\longrightarrow} \begin{gathered}
\text { ZMw/o } \\
0,15 \mathrm{mins}
\end{gathered}
$$

B

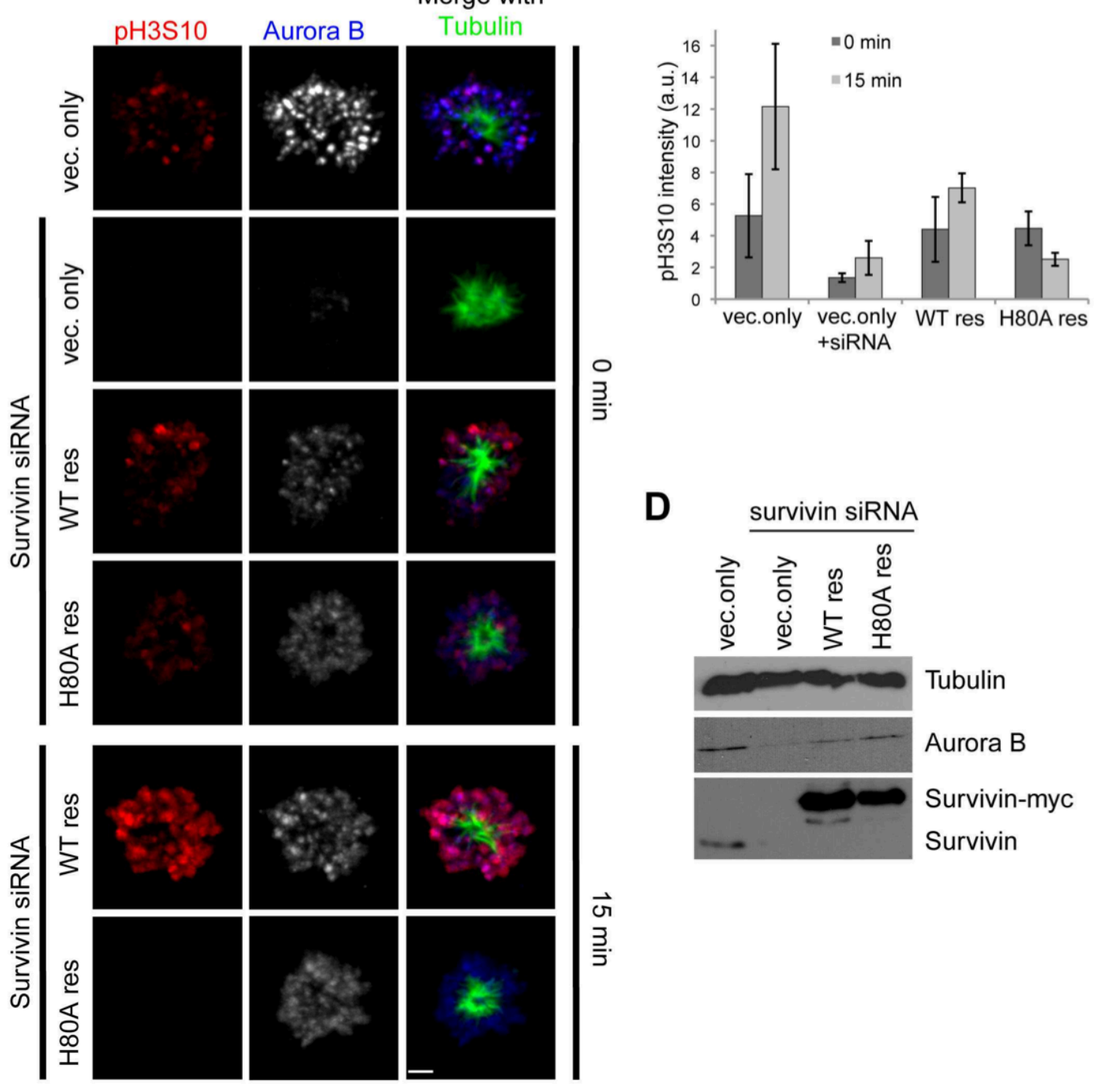

Figure 2-8 
Supplemental figure 2-5: Microtubules regulate spreading Aurora B activity from centromeres to chromosome arms.

A) PLA controls for Fig. 2-5. Bar-13um. B) Scheme representing the timeline of ZMwashout assay to measure spreading of activity that is used in Fig. 2-6 G and Fig. 2-7

A,B. C) Scatter plot showing correlation of total tubulin intensities and total phospho$\mathrm{H} 3 \mathrm{~S} 10$ in individual HeLa cells at the indicated time points in the experiment shown in Fig. 2-7 D and outlined in Suppl. Fig. 2-5 B. $\mathrm{R}^{2}$ values are mentioned in respective colors. 
A
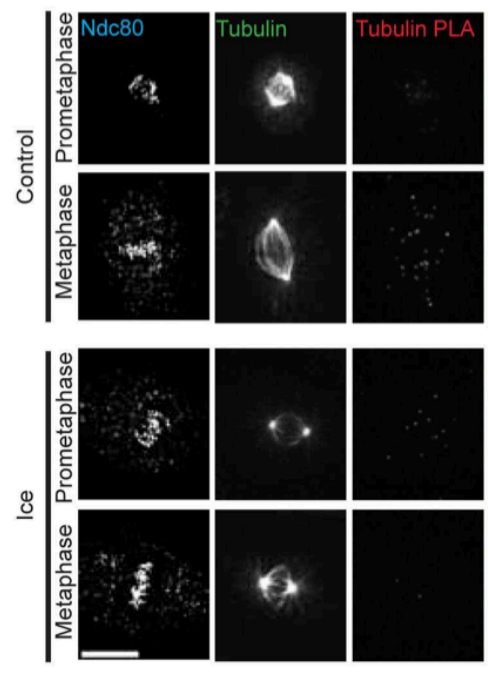

C

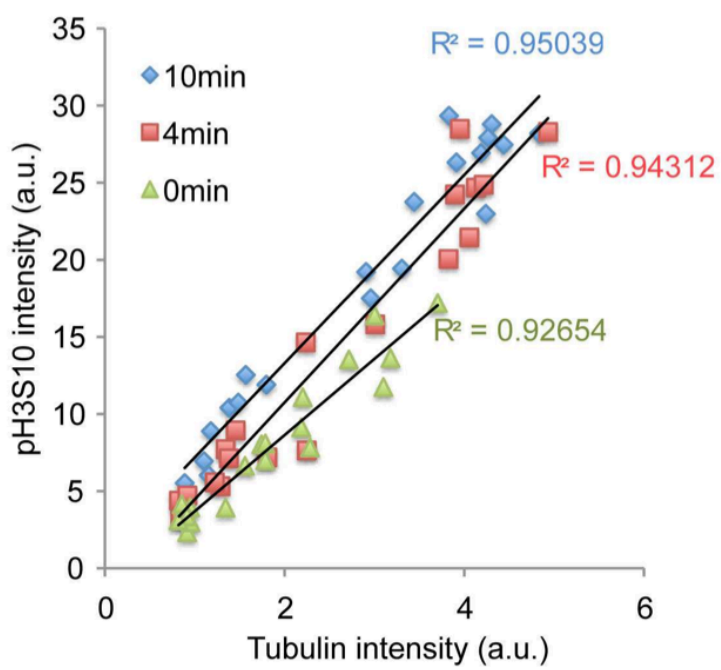

B

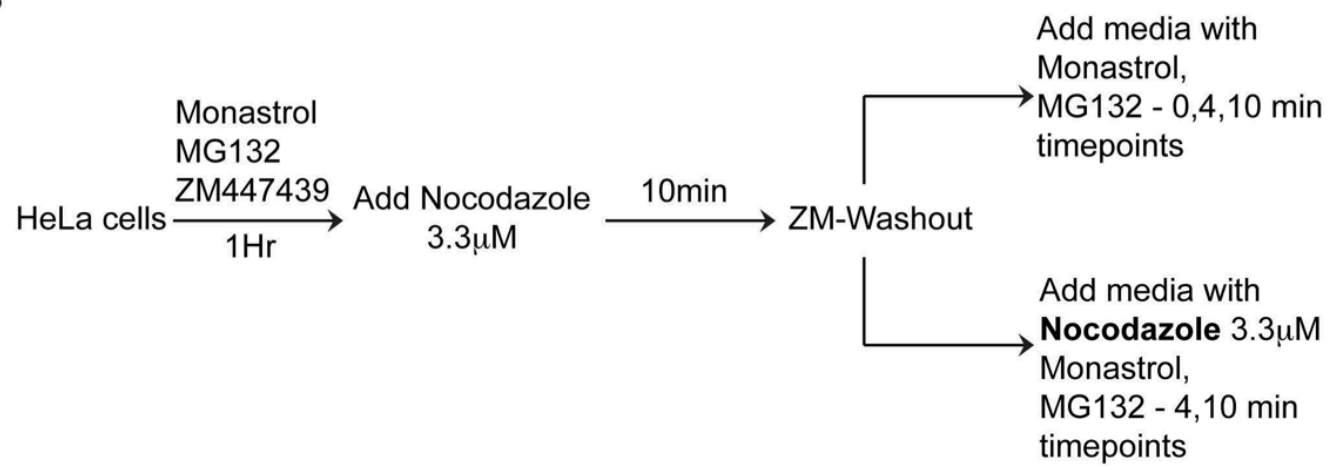

Supplemental Figure 2-5 
Interdependence of kinetochore axis and cohesion axis through Aurora B

Next we wanted to test if the kinetochore axis and the cohesion axis were working independently of each other or not. We already showed that EB1 depletion affects both axis and this can be rescued by expressing CenpB-INCENP, which binds alpha satellite DNA and thus can recruit Aurora B directly to the inner centromere in the absence of the histone phosphorylation marks. We used the same method to ask if the two feedback loops (Saurin et al., 2011; Wang et al., 2011b) were only responsive to Aurora B stimulation or recruitment of Bub1 depended on Haspin activity. Haspin inhibitor treatment in U2OS cells showed reduced kinetochore levels of Mps1, Bub1, pH2AT120 and Sgo1 (Fig. 2-9). We found expression of CENPB-INCENP in Haspin inhibitor treated cells rescued the recruitment of Bub1 (Fig. 2-9 A-C). We also checked the effect on other members of the kinetochore axis and observed that it was consistent for phospho-H2AT120, Sgo1 and Mps1 (Fig. 2-9 D,E). These data suggests that the only common component connecting the two feedback loops is Aurora B. EB1 / microtubule dependent recruitment and activation of Aurora B triggers both the loops to allow further enrichment of CPC. 
Figure 2-9: Kinetochore axis and cohesion axis are connected through centromeric Aurora B.
A) Expression of CENPB-INCENP fusion protein in U2OS cells rescued the reduction of Bub1 and pH2AT120 levels after Haspin inhibitor treatment. A stable U2OS line was either mock treated (control) or EB1 siRNA treated with or without CENPB-INCENP induction by doxycyclin. Bar-1.8um. B) Quantification of Bub1 levels at the indicated treatment conditions. C) Western blot showing depletion of EB1 by siRNA and expression of CenpB-INCENP fusion protein. D) Immuno-fluorescence staining of similarly treated U2OS cells as in A showing pH2AT120, Sgo1 and Mps1. 
A
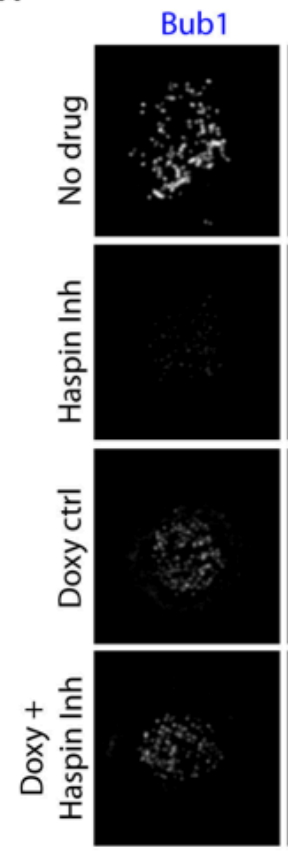

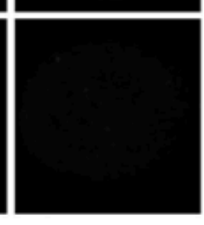

Merge
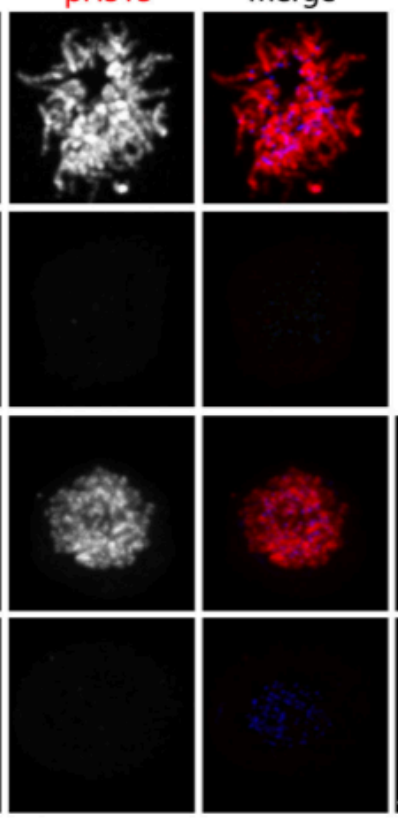

Merge with CenpB-INCP

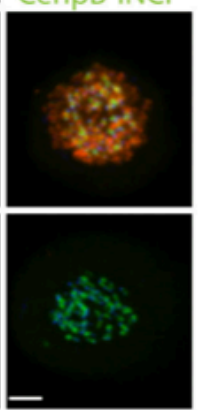

B

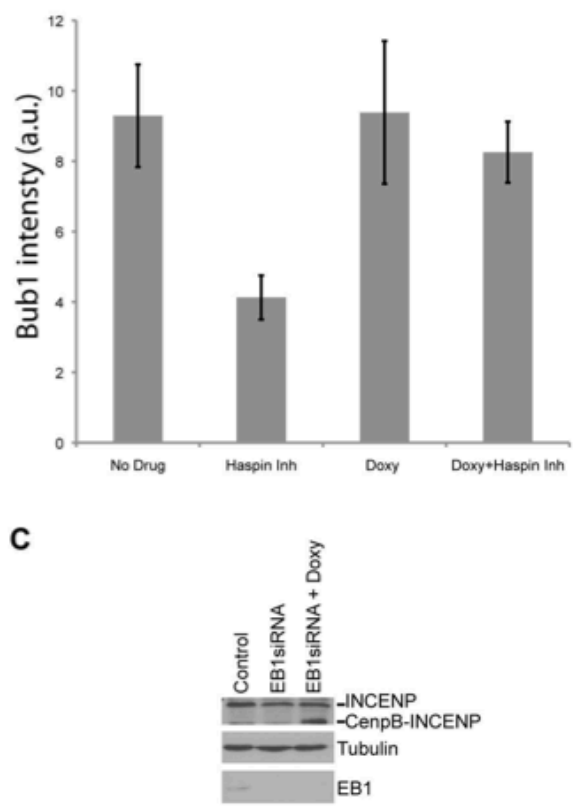

D

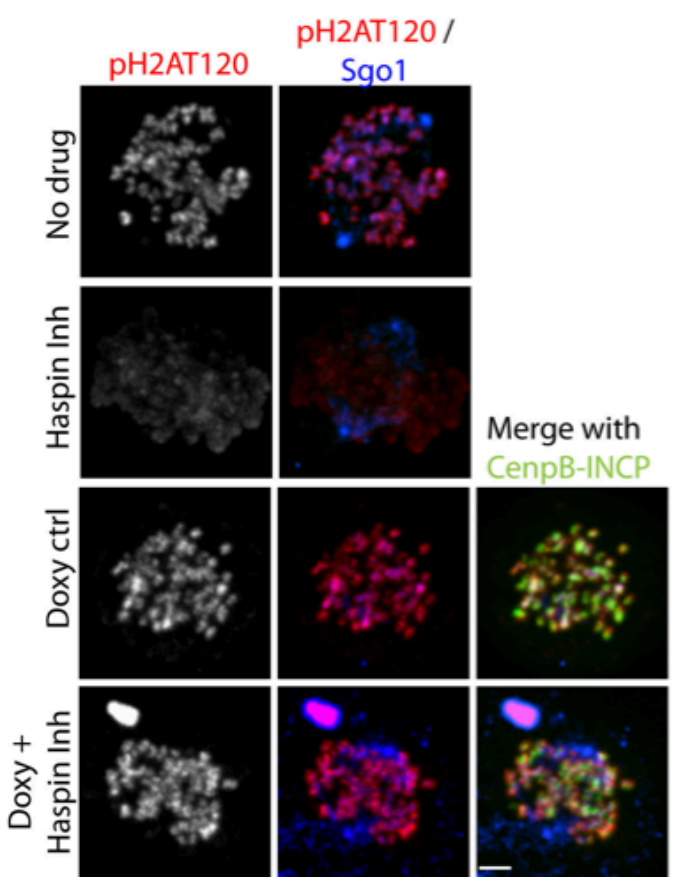

E

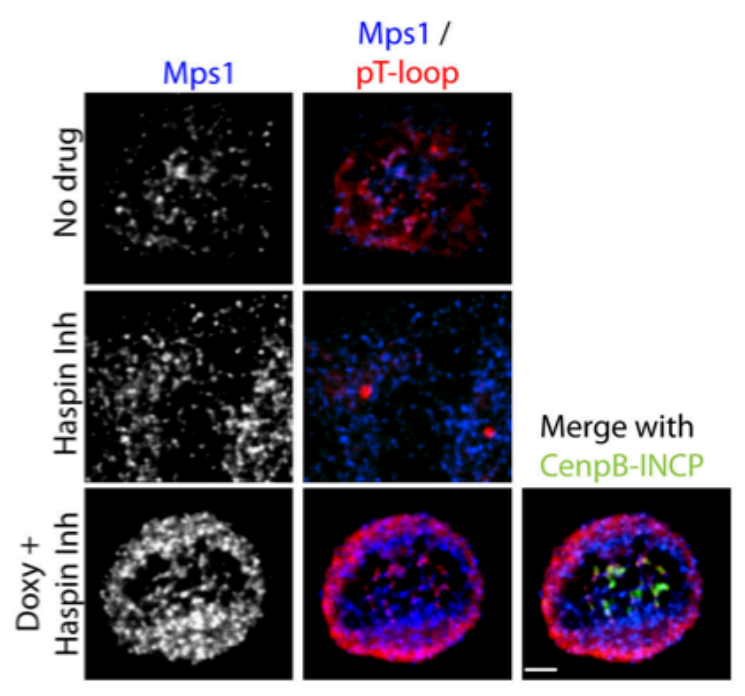


EB1 is required for the Aurora B anaphase gradients

During anaphase Aurora B kinase phosphorylates chromatin in the region between the midzone microtubules and the presumptive cytokinetic furrow (Fuller et al., 2008). Microtubules are required to activate Aurora kinase in anaphase (Fuller et al., 2008). To determine if EB1 depletion disrupts anaphase Aurora B activity we measured phospho-H3S10 levels on lagging chromosomes in early and late anaphase. Since EB1 depletion triggers the spindle checkpoint to arrest cells we also treated HeLa cells with Mad2 siRNA (Fig. 2-10 B). This generated anaphase-lagging chromosomes, which enabled us to visualize the anaphase gradients of phospho-H3S10 activity. As expected, we saw high phospho-H3S10 levels on the lagging chromosomes in Mad2 siRNA treated cells and activity decreased as a function of lateral distance from the midzone center. Cells treated with both EB1 and Mad2 siRNAs had reduced Aurora B activity on anaphase chromatin, with minimal to no difference in phospho-H3S10 levels on sister chromatids near the poles when compared to lagging chromosomes in the spindle center (Fig. 2-10 A). The effect of EB1 depletion on disrupting the anaphase gradient is observed in late anaphase. Line scans elaborate the disruption of anaphase chromatin phosphorylation in EB1 depleted cells (Fig. 2-10 C,C'). Furthermore, the ratio of phospho-H3S10:chromatin staining clearly shows the central gradients of Aurora B activity, as well as its dependence on EB1 (Fig. 2-10 D). This is consistent with the role of Bimlp regulation of Ipl1 in budding yeast anapahse (Zimniak et al., 2009). 
Figure 2-10: EB1 depletion disrupts Aurora B anaphase gradient
A) HeLa cells transfected with Mad2 siRNA or Mad2 and EB1 siRNA for 48 hours stained with pH3S10 to visualize the anaphase gradients of Aurora B activity on anaphase-lagging chromosomes. B) Western blot of cell lysate treated with respective siRNAs, similarly as in A, showing Mad2 and EB1 depletion. C) Linescan showing intensities along line shown in the merge of the Mad2 depleted cell in $\mathrm{C}$ top or bottom $\left(\mathbf{C}^{\prime}\right)$. Bar $=2.7 \mu \mathrm{m}$. D) Ratio of pH3S10 intensity to chromatin staining is high at midzone center in Mad2 siRNA treated cells but not in EB1\&Mad2 siRNA treated cells. 
A

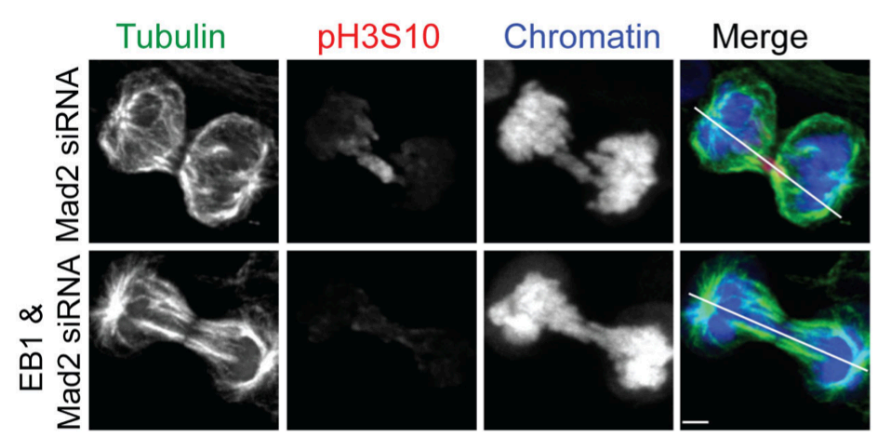

C'

C

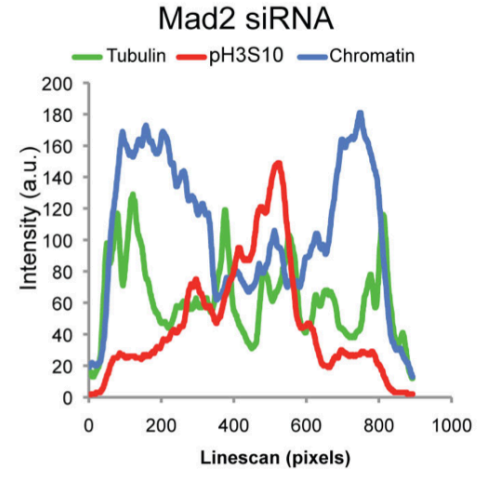

B

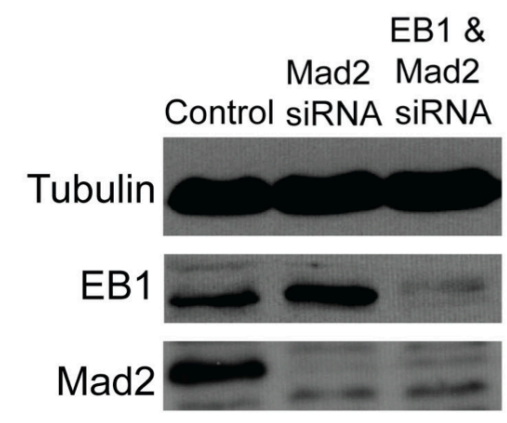

EB1 \& Mad2 siRNA

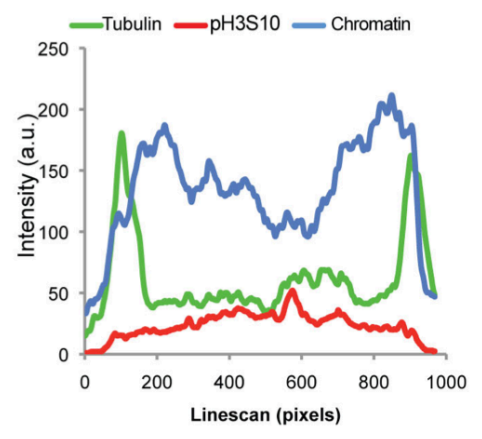

D

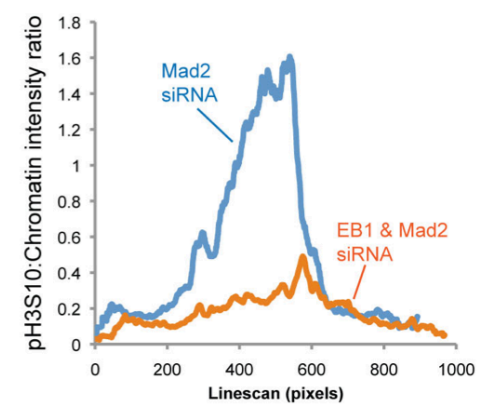

Figure 2-10 


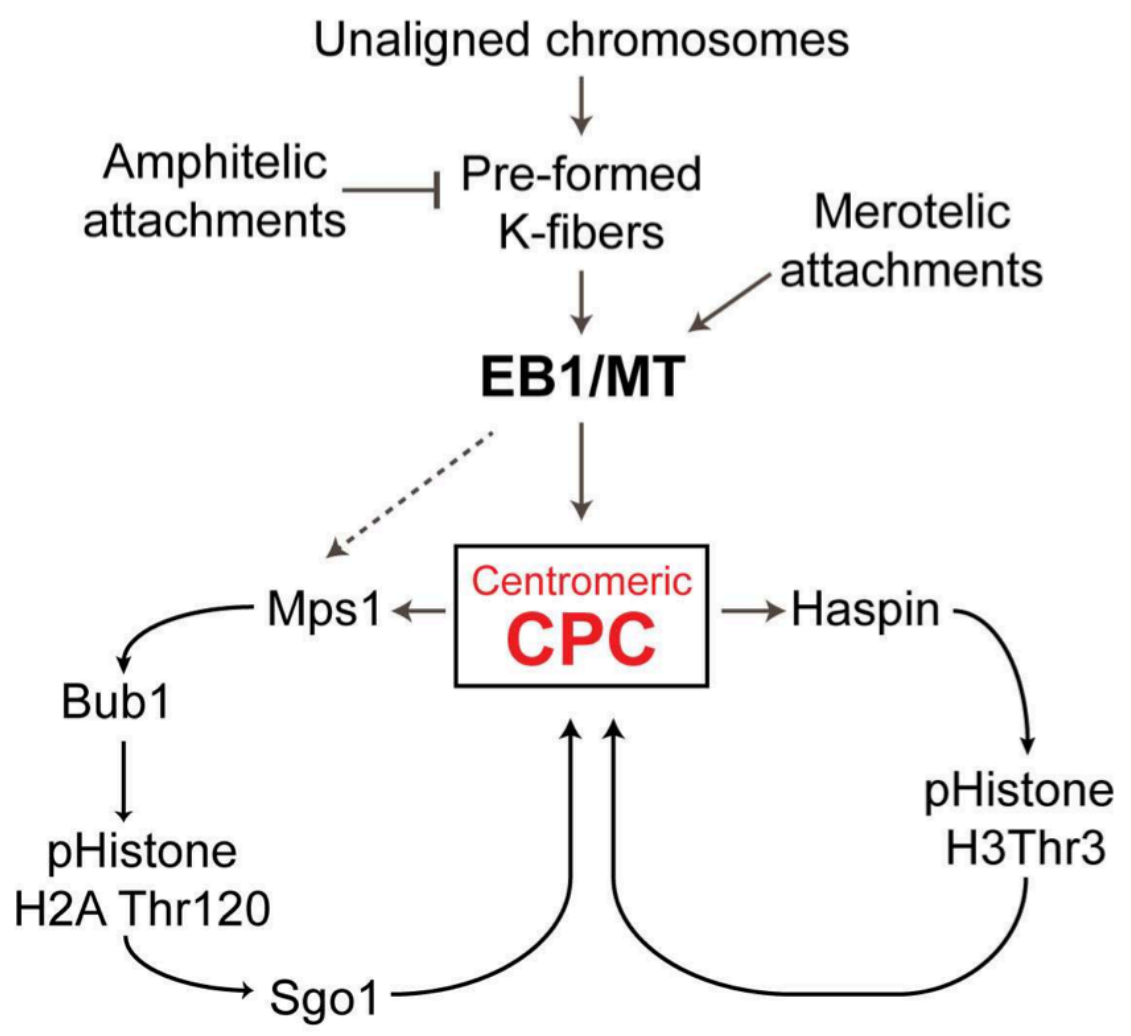

Figure 2-11: Cooperation of histone phosphorylation and microtubules in centromeric localization of Aurora B

Model depicting the role of EB1 and microtubules in regulating inner centromeric Aurora $\mathrm{B}$ and its activity. 


\section{Discussion}

We have demonstrated that EB1 and microtubules play important roles in the recruitment of the $\mathrm{CPC}$ to inner centromeres after, but not before, nuclear envelope breakdown. We show that EB1 and microtubules are in the same pathway. In the absence of EB1 or microtubules we still find pools of Aurora B at inner centromeres but the levels are highly enriched by EB1 and microtubules. EB1 and microtubules are also essential for spreading Aurora B activity from inner centromeres to both kinetochores and to chromosome arms. EB1 has been previously shown to co-immunoprecipitate with the CPC and to block PP2A activity against Aurora B (Sun et al., 2008). Our data are consistent with these observations. While we can detect a weak direct interaction of EB1 with Aurora B, we don't see any direct effect on kinase activity (Fig. 2-4 B,C). Thus we suggest that one role of EB1 is to inhibit PP2A to prolong Aurora B activity so that it can concentrate at centromeres by triggering the feedback loops and then spread to distant substrates.

Aurora B phosphorylates substrates at the centromere in cells treated with nocodazole. How then can we argue that microtubules regulate Aurora B? We find Aurora B levels at the inner centromeres drop significantly in $3.3 \mu \mathrm{M}$ nocodazole (Fig. 2$3 \mathrm{~A}, \mathrm{~B})$, but there is still a pool that localizes in a microtubule independent manner. This pool is dependent upon the histone phosphorylation pathways and is further reduced by simultaneous inhibition of Aurora B activity by ZM (Fig. 2-6 G), suggesting that there are at least two pathways that localize Aurora B. Our data suggest that EB1/microtubule stimulation is upstream and activate the histone phosphorylation loops to generate increased levels of CPC at centromeres. These redundancies and positive feedback loops 
complicate the study of the CPC and it is critical to knock out one pathway in order to study the regulation of a second pathway.

How do EB1 and microtubules localize the CPC? We show direct stimulation of kinase activity by microtubules and direct interaction of Aurora B and EB1. Moreover, EB1(K89E) microtubule binding mutant is unable to enrich CPC at the inner centromeres in EB1 depleted cells. The loss of EB1 also reduces, phospho-HistoneH3T3, Bub1 at kinetochores and Bub1 phosphorylation of Histone $\mathrm{H} 2 \mathrm{~A}$, which are all required to localize the CPC to centromeres (Wang et al., 2010; Kelly et al., 2010; Yamagishi et al., 2010; Niedzialkowska et al., 2012; Du et al., 2012; Jeyaprakash et al., 2011). The simplest model is that EB1 on microtubule plus-ends enrich Aurora B at inner centromeres. This triggers positive feedback loops that regulate the histone kinases (Fig. 2-3 and Fig. 2-11) (Wang et al., 2011b; van der Waal et al., 2012). The Mps1 kinase is also stimulated by microtubules and enhances phospho-HistoneH2A levels to rapidly recruit Aurora B to centromeres (van der Waal et al., 2012; Stucke et al., 2004). We postulate that the EB1/microtubule pathways modulate the amount of $\mathrm{CPC}$ at centromeres, while the histone phosphorylation pathways ensure that the $\mathrm{CPC}$ can only be stimulated at inner centromeres where the two histone marks intersect.

There are distinct types of microtubules in the spindle and our data suggest that the major pool of microtubules that activate the CPC recruitment in prometaphase are the PreK-fibers that are nucleated by kinetochores (Khodjakov et al., 2003; Platani et al., 2009; Mitchison and Kirschner, 1985). This is supported by our observation that there are higher levels of Aurora B at the microtubule foci near kinetochores in cells treated with $0.33 \mu \mathrm{M}$ but not $3.3 \mu \mathrm{M}$ nocodazole (Fig. 2-3 A,B and Suppl. Fig. 2-3 A). In addition, 
PLA suggests that Aurora B-tubulin interactions and Aurora B-EB1 interactions are highest at centromeres in early prometaphase cells and that Aurora B can be found on PreK-fibers (Fig. 2-4 E and Fig. 2-5 B,C). These conclusions are also supported by earlier work that showed that the $\mathrm{CPC}$ requires both chromosomes and microtubules for proper function and organizes PreK-fibers (Tseng et al., 2010; Tulu et al., 2006). Finally, in the accompanying paper (Matson et al., submitted for publication) we show that centromeres with PreK-fibers have 3-fold more Aurora B and 4-fold more kinetochore activity than centromeres without PreK-fibers in the same cell. We found a subpool of Aurora B on astral microtubules and a second pool on K-fibers of metaphase aligned chromosomes. The function of the astral microtubule pool is still unclear but we speculate that it is involved in spreading Aurora kinase activity from inner centromeres to distant substrates such as chromosome arms. As we will discuss below, we postulate that EB1 may specifically activate the kinase on antipoleward K-fibers in metaphase to break the asymmetry of sister kinetochores.

\section{Microtubules/EB1 allow the CPC to communicate with the spindle}

Microtubule/EB1 regulation of Aurora B levels provides a mechanism for spindle status to regulate inner centromere signaling. We will outline three examples from the literature that could be explained by this regulation.

EB1 is the only protein that localizes specifically to the sister kinetochores that have the growing microtubule ends (anti-poleward) (Tirnauer et al., 2002). This finding combined with our data suggest that Aurora B activity would be higher on the antipoleward sister kinetochores. Consistent with this idea most PLA signals are typically 
found near one of the two sister kinetochores (Fig. 2-4 E,F). Differential phosphorylation on the poleward and anti-poleward sides could provide mechanisms to coordinate the two sisters to allow proper chromosome movements (Liu et al., 2009; Tirnauer et al., 2002; Dumont et al., 2012).

Aurora B is recruited specifically to merotelic attachment points and Aurora B activity is required to resolve merotelic attachments (Ruchaud et al., 2007; Knowlton et al., 2006; Lan et al., 2004; Cimini et al., 2006; Kallio et al., 2002; Lampson et al., 2004; Liu et al., 2009). Our findings can in part explain these phenomena. A defining feature of a merotelic attachment is the presence of microtubules near or adjacent to inner centromeres. We suggest that the presence of microtubules recruits additional Aurora B to resolve merotelic attachments. In addition a merotelic attachment brings kinetochores close to inner centromeres to allow efficient phosphorylation of kinetochore substrates such as Hec1 and Ska that release microtubule attachments (Terada, 2001; Deluca et al., 2006; Wheatley et al., 2001a; Welburn et al., 2010; Cheeseman et al., 2006).

Centromeric Aurora B levels decrease as chromosomes become aligned at the metaphase plate in non-transformed human cells (Lan et al., 2004; Salimian et al., 2011; Knowlton et al., 2006; Cimini et al., 2006; Kallio et al., 2002; Liu et al., 2009). We suggest that microtubule stimulation of Aurora B recruitment could also underlie this phenomenon. Kinetochores nucleate microtubules because the Nup160 complex recruits the $\gamma$-TURC complex and the TPX2 protein may also nucleate microtubules (Wang et al., 2010; Mishra et al., 2010; Kelly et al., 2010; Orjalo et al., 2006; Yamagishi et al., 2010; Niedzialkowska et al., 2012; Du et al., 2012; Jeyaprakash et al., 2011). Interestingly, these activities are inhibited by high RanGTP levels (Yamagishi et al., 2010; Mishra et 
al., 2010). We suggest that the concentration of chromosomes on the metaphase plate could generate high RanGTP, decrease kinetochore nucleated microtubules and downregulate CPC recruitment. However, using live cell imaging of metaphase cells it has been shown that brief treatments with nocodazole actually increases CPC recruitment (Tsukahara et al., 2010; Salimian et al., 2011; Wheatley et al., 2004; Chu et al., 2011). While this experiment initially seems to contradict our findings, we note that nocodazole takes at least 10 minutes to fully depolymerize microtubules and in the short movies that were performed the nocodazole will decrease the stability of k-fibers and could increase the PreK-fibers that stimulate Aurora B localization (Fig. 2-3 A) (Salimian et al., 2011; Brito et al., 2008). PreK-fiber activation allows Aurora B activity to be higher in prometaphase to generate SAC signaling and regulate Hecl dependent "end-on" attachment and favor dynein dependent lateral attachments (accompanying paper Matson et al. submitted for publication). Thus new models for kinetochore regulation have emerged from integrating the regulation of Aurora B by microtubules into the key functions of Aurora B.

We have also identified conditions that reveal global regulation of the histone code by microtubules. Specifically the amount of Histone H3Ser10 phosphorylation is both spatially and quantitatively correlated with the amount of microtubules after washout of ZM and nocodazole (Fig. 2-7 D). Although still speculative this suggests a previously unappreciated role of microtubules and EB1 in regulating a histone modification throughout chromatin. It will be important to determine if mitotic spindle structure influences gene transcription and/or the maintenance of chromatin state in the following interphase. 


\section{Materials and Methods}

Kinase activity assay

Recombinant $\mathrm{AI}^{(790-856)}$ purified from BL21 bacterial expression system as previously described (Sessa et al) was diluted to the required concentrations and incubated with either $3 \mu \mathrm{M}$ Taxol-stabilized microtubules (prepared as in (Desai et al., 1999) or 1XBRB80 (80 mM Pipes, $1 \mathrm{mM} \mathrm{MgCl} 2$, 1mM EGTA pH-6.8 with $\mathrm{KOH}$ ), for 15 minutes. Xenopus EB1 was purified from BL21 bacterial expression system from a pET28c-EB1 vector. Assays were carried out in kinase buffer (20mM Tris- $\mathrm{HCl} \mathrm{pH}-7.5,1 \mathrm{mM} \mathrm{MgCl}_{2}$, $25 \mathrm{mM} \mathrm{KCl}, 1 \mathrm{mM}$ DTT and $100 \mu \mathrm{M}$ ATP $/ 1 \mu \mathrm{M} \gamma^{32} \mathrm{P}$-ATP mix) with myelin basic protein (MBP, Invitrogen), as substrate. Activity was initiated by incubation with microtubules/1X BRB80 and stopped after 2 minutes by adding SDS-PAGE sample buffer. Samples were separated on 15\%-SDS-PAGE gel, stained with coomassie blue, dried on Whattman paper together with aliquots of $\gamma^{32} \mathrm{P}$-ATP and exposed to Phosphor Screen (Molecular Dynamics) overnight. Phosphor Screens were scanned on a Storm-860 Phosphor-scanner (Molecular Dynamics) and resulting images were processed and quantified using ImageQuant (Molecular Dynamics) to calculate amount of $\mathrm{PO}_{4}^{3-}$ incorporated on MBP. Error bars represent standard deviations.

\section{Cell lines and Plasmids}

Wildtype or K89E mutant EB1 was cloned into DLAP destination vector, which is derived from pcDNA5.0/FRT vector (Invitrogen) with C-terminal dual tags of S peptide and GFP (pDLAP-EB1). The clones were modified by site directed mutagenesis to make them resistant to the coding sequence targeted siRNA (pDLAP-EB1siRes and 
EB1K89EsiRes). Flp-in HeLa-TRex cells (Tighe et al., 2008) were co-transfected with pDLAP-EB1 and pOG44. Stable lines expressing (WT)EB1-LAP were subsequently created by selection with Hygromycin. U2OS-TR stable cell line with doxycycline inducible CenpB-INCENP fusion protein was a kind gift from Sussane Lens (Saurin et al., 2011).

\section{Tissue culture and Transfection}

HeLa T-Rex cells (Invitrogen) were grown and passaged in Dulbecco's Modified Eagle's Medium (DMEM) supplemented with 10\% Fetal Bovine Serum (FBS). Xenopus S3 cells were cultured in 66\% L-15 media (Sigma) supplemented with 10\% FBS, $100 \mathrm{IU} / \mathrm{ml}$ penicillin, $100 \mathrm{ug} / \mathrm{ml}$ streptomycin and $1 \mathrm{nM}$ sodium pyruvate at $18^{\circ} \mathrm{C}$. HeLa cells were plated at $25 \%$ confluency onto poly-L-Lysine coated $18 \mathrm{~mm}$ coverslips in 12 -well dish (Corning) overnight. siRNA transfection for EB1 and Mad2 knockdown were done using Lipofectamine RNAiMAX (Invitrogen) according to manufacturer's protocol and survivin knockdown was done using Oligofectamine (Invitrogen) as previously described (Niedzialkowska et al., 2012). HeLa cells were treated with EB1 siRNAs (Custom siRNA - Thermo/Dharmacon; 5' - AAgUGAAAUUCCAAGCUAAGCUU - 3'; Custom 3'UTR siRNAs - 5' - GAATGCTGGAGAGATGTTATG - 3' and 5' GCACTAATCTCTTTGGAGA - 3') at 10nM and a SMARTpool of siRNA oligos against Mad2 (Thermo/Dharmacon - L-003271-00-0005) with a final concentration of $20 \mathrm{nM}$, either separately or in combination for 48 hours. pDLAP-EB1 (WT or siRes) was transiently transfected with Lipofectamine2000 (Invitrogen) following manufacturer's protocol, 24 hours after siRNA transfection, for rescue experiments. Cells grown in 12- 
well dish were transfected with 250ng (500ng for 6-well dish) plasmid and fixed for immunofluorescence after 24 hours.

\section{Immunoblotting}

HeLa cells from 2-wells of 6-well dish were scraped and spun down at $1500 \mathrm{rpm}$ to generate cell lysates for western blotting. Pellets were washed with $1 \mathrm{X}$ DPBS (Invitrogen) and resuspended in $2 \mathrm{X}$ SDS sample buffer, sonicated and run on SDSpolyacrylamide gel. Antibodies used were as follows: anti-survivin (Cell Signalling), anti-Tubulin DM1 $\alpha$ (Sigma), anti-AIM1 (BD), anti-EB1 (BD), anti-Mad2 (Bethyl), anti$\operatorname{Bub1}($ Abcam).

\section{ZM-washout assays}

HeLa cells were plated at $75 \%$ confluency onto poly-L-Lysine coated $18 \mathrm{~mm}$ coverslips in 12-well dish (Corning) overnight. Cells were treated with $2 \mu \mathrm{M}$ ZM447439 (Enzo), $42 \mu \mathrm{M}$ MG132 (Tocris) and $100 \mu \mathrm{M}$ Monastrol (Tocris) for 1 hour in DMEM $+10 \% \mathrm{FBS}$ at $37^{\circ} \mathrm{C}$. Cells were either fixed or to assay recovery of Aurora B activity after ZM-washout they were washed with $1 \mathrm{X}-\mathrm{DPBS}$ for three times and replaced in fresh DMEM+10\%FBS with $42 \mu \mathrm{M}$ MG132 and $100 \mu \mathrm{M}$ Monastrol. ZM+Nocodazole washout assay: After 60 minutes in ZM447439/MG132/Monastrol, nocodazole was added to a final concentration of $3.3 \mu \mathrm{M}$ and kept at $37^{\circ} \mathrm{C}$ for 10 minutes. Cells were either fixed at this point or shifted to fresh DMEM+10\%FBS with $42 \mu \mathrm{M}$ MG132 and $100 \mu \mathrm{M}$ Monastrol with or without $3.3 \mu \mathrm{M}$ nocodazole. 


\section{Proximity Ligation Assay}

Xenopus S3 cells were grown on acid washed, poly-lysine coated coverslips affixed with a silicone gasket (Grace Bio-Labs, Inc.). To destabilize microtubules (Fig. 2-5 A) cells were washed into ice-cold growth media and incubated in an ice water bath for 5 minutes immediately before fixation. Untreated control cells were fixed simultaneously.

Monastrol treatment (100uM) was done for $1 \mathrm{hr}$ and fixed with 4\% paraformaldehyde in $1 \mathrm{x}$ PHEM at RT for 20 min. Coverslips were washed 3 times with TBS $+0.05 \%$ Tween and stored at 4 degrees until ready for use in PLA. Nocodazole washout - Xenopus S3 cells were treated with $2 \mathrm{uM}$ nocodazole or an equivalent dilution of DMSO for $1 \mathrm{hr}$. Cells were either left in DMSO, 2 uM nocodazole or washed three times with fresh media and released for 5, 10 or 15 min. prior to fixation with 4\% paraformaldehyde. Coverslips were washed 3 times with TBS $+0.05 \%$ Tween and stored at 4 degrees until ready for use in PLA.

PLA was performed after fixation using Duolink II PLA probes anti-Mouse MINUS and anti-rabbit PLUS and Duolink II Detection Reagents Orange (Olink Bioscience). The assay was performed following the manufacturer's recommended protocol using the provided blocking solution and antibody diluent. Samples were incubated in primary antibodies overnight at $4{ }^{\circ} \mathrm{C}$. The following primary antibodies were used at the indicated dilutions: anti-xlAurora B (1:400; P.Todd Stukenberg, University of Virginia), anti- $\beta$ Tubulin, AA2 (1:500; UVA Lymphocyte Culture Center), anti-hEB1 (1:500; BD Transduction Labs), and anti-hAurora B (1ug/ ml, Abcam,) 


\section{Immunofluorescence}

HeLa cells were fixed with $100 \%$ methanol for EB1 immuno-staining and PLA experiments (anti-EB1 antibody - BD). All other immuno-staining and PLA experiments were done after co-fixing cells with 4\%-paraformaldehyde, 1X PHEM (60mM PIPES, 25mM HEPES, 10mM EGTA, 4mM $\mathrm{MgCl}_{2}$, $\mathrm{pH}-6.9$ ) and 0.5\% TX-100 for 20 minutes. Immunofluorescence staining was done in 3\% BSA/TBS- $0.05 \%$ Tween-20 using these antibodies: anti-Tubulin DM1a (Sigma), anti-phosphoH3Ser10 (Millipore), antiphospho-H3T3 (Millipore), anti-phospho-H2AT120 (Activ Motif), phospho-KNL1(S24 \& S60) (Iain M. Cheeseman, (Welburn et al., 2010), anti-AIM1 (Aurora B) - ( BD Transduction Labs), ACA (Antibodies Incorporated), anti-hEB1 (BD Transduction Labs) and To-Pro3 (Invitrogen).

Following the PLA procedure, cells were incubated in $10 \%$ normal mouse serum and $10 \%$ normal rabbit serum (Jackson ImmunoResearch Laboratories, Inc.) in 3\% $\mathrm{BSA} / \mathrm{TBS}+0.05 \%$ Tween-20 for 30 minutes at room temperature to block any open binding sites on the PLA probes. FITC conjugated anti- $\alpha$-Tubulin, DM1 $\alpha$ (Sigma) and Alexa Fluor 647 conjugated polyclonal antibodies ( $x$ INdc80, $x l$ INCENP or hBorealin) were utilized for co-staining with PLA reactions. Alexa-647 polyclonal antibody conjugations were prepared using an Alexa Fluor ${ }^{\circledR} 647$ labeling kit following the manufacturer's recommended protocol (Invitrogen).

Fluorescence Microscopy, Image Acquisition and Processing

Images were captured using a Zeiss Axiovert 200 inverted microscope fitted with a confocal scanner using a krypton/argon laser (Perkin Elmer), Hamamatsu EMCCD 
camera, a NanoScanZ motor (Prior) and a 63X and 100X oil Plan-Apochromat objective. An acousto-optic tunable filter (AOTF) was used for detection of light at 488, 568 and $647 \mathrm{~nm}$. Photographs were taken as z-series with $0.4 \mu \mathrm{m} \mathrm{Z}$ - steps. All aspects of image acquisition and processing were controlled by Volocity software (Perkin Elmer). Images from the same experiment were captured using identical acquisition settings and the contrast enhancement tool (Volocity®5.5 Perkin Elmer or ImageJ-NIH) was used to scale the images to the same black and white values.

Total sum intensity in each cell was determined by drawing regions of interest (ROIs) around individual cells with the freehand or lasso tool. To measure intensities specifically at kinetochores or centromeres a volume thresholding algorithm was made for each channel. ROIs picked up by the algorithm were manually confirmed as kinetochores or centromeres by comparing with ACA. Here it is important to mention that the algorithm picked voxel volumes above a certain intensity. This resulted in some underestimation of severity of loss of signal after treatments for signals that were not detectable above background. This was considered reasonable, as the changes were still highly significant. Background intensity per $\mu \mathrm{m}^{3}$ was calculated for each image by drawing a ROI and dividing the sum intensity of the ROI by its volume. This value was multiplied by the volume of the ROI drawn by freehand tool or picked up by the algorithm and subtracted from the sum intensity measurement for the cell/ kinetochore to find the corrected sum intensity. Whenever we used the volume thresholding algorithm we divided the corrected total sum intensity per voxel volume by the voxel volume (intensity $/ \mu \mathrm{m}^{3}$ ) and referred to it as arbitrary units (a.u.). This was done to avoid error in estimation of absolute centromeric intensities in cases where the voxel failed to 
distinguish between two or more closely situated centromeres. Error bars represent standard deviations unless mentioned otherwise. 


\section{Acknowledgement}

We would like to thank Iain M. Cheeseman for the gift of the phospho-KNL1 antibody, Stefan Bekiranov and Stephen Hoang for help with statistical analysis, Sussane Lens for lending us U2OS-TR stable cell line with doxycycline inducible CENPB-INCENP fusion protein, D.J. Burke, D.R. Foltz, M. Mitchell Smith and Michael A. Lampson for critical review of the manuscript. This work was supported by grants from the NIH (GM063045) to P.T. Stukenberg and in part by a gift provided to the University of Virginia by Altria, USA to B.B. and P.T.S. and B. Banerjee is supported by NIH training grant (GM063045) and Robert R. Wagner Fellowship Fund. B. Banerjee, C. A. Kestner and P.T. Stukenberg designed and executed the experiments and B. Banerjee and P.T. Stukenberg wrote the manuscript. 


\author{
Abbreviations List \\ CPC - Chromosomal Passenger Complex \\ MT - Microtubules \\ PreK-fibers - pre-formed K-fibers \\ ZM - ZM447439 Aurora B inhibitor \\ Noc - Nocodazole \\ HI - 5-iodotubercidin (Haspin inhibitor)
}


CHAPTER III:

IN VITRO BIOCHEMICAL CHARACTERIZATION OF THE CHROMOSOMAL PASSENGER COMPLEX 


\begin{abstract}
The chromosome passenger complex (CPC) is a critical integrator of signals from multiple sources in mitosis. It is recruited to the inner centromeres in prometaphase where it corrects kinetochore microtubule attachment errors and signals the spindle checkpoint. It is important to regulate Aurora B activity appropriately at this stage. We demonstrate that Aurora $\mathrm{B}$ has the ability to auto-activate kinase activity in a concentration dependent manner and this requires a domain outside the catalytic subunit. We identify cofactors like microtubules and DNA that allow local enrichment of CPC, which triggers the stimulation of kinase activity. We also show that key residues in the amino-terminal domain can regulate kinase activity in response to auto-phosphorylation. Finally we show that the catalytic subunit has a microtubule-binding domain and its affinity for microtubules is regulated by auto-phosphorylation. These data suggests that Aurora B kinase activity is not merely turned ON or OFF but is tightly regulated to allow CPC to respond to changes in its immediate environment during mitosis.
\end{abstract}




\section{Introduction}

The chromosome passenger complex regulates a wide spectrum of events during mitosis (Ruchaud et al., 2007). For this it is very important that the CPC reaches specific locations through the course of mitosis. The previous chapter elaborates the various pathways that ensure that this happens with very high fidelity. Aurora B is unique because it does not operate in typical ON or OFF states but is tightly regulated by its immediate environment. In this chapter we will focus on elaborating how the Aurora B kinase activity is regulated by itself and a group of co-factors that allow targeting of specific pools of substrates.

\section{Divergent $N$-terminal region in Aurora kinases}

Aurora B kinase is one of 3 Aurora kinases in mammals. Most vertebrates have at least 2 Aurora kinases. One or more orthologues are found in yeast, flies, worms and other invertebrates. The three mammalian Aurora paralogues are very similar in sequence, a conserved kinase domain, in which human Aurora A and B share $71 \%$ identity. They even share the same target consensus motif for substrate phosphorylation (K/R)-(K/R)-X-(S/T)-(I/L/V) (Cheeseman et al., 2002). However, the three Auroras differ in the length and sequence of their amino-terminal domain (Bischoff et al., 1998). This domain's uniqueness is critical to Aurora kinases A and B as they interact with specific allosteric cofactors through the N-terminal lobe that activate the kinase (Bishop and Schumacher, 2002; Eyers et al., 2003; Sessa et al., 2005; Tsai et al., 2003; Honda et al., 2003; Adams et al., 2000). This also allows them to localize to distinct sub-cellular domains and target mutually exclusive set of substrates to regulate different mitotic 
processes. We explored the possibility that the N-terminal tail of Xenopus Aurora B might be more than just a unique docking site for INCENP and play an auto-regulatory role for the kinase.

Co-factors that regulate Aurora B kinase activity

Phosphorylation of INCENP by Aurora B on its C-terminal TSS motif is part of a feedback loop that activates the kinase (Bishop and Schumacher, 2002; Sessa et al., 2005). Phosphorylation of these residues in the IN-Box (Adams et al., 2000) leads to 7fold stimulation of kinase activity as compared to Aurora B phosphorylated on its activating T-loop (Sessa et al., 2005). Chicken DT40 cells expressing INCENP TSS motif mutants (INCENP ${ }^{\mathrm{TAA}}$ ) show spindle elongation defects and failure to complete cytokinesis (Xu et al., 2010). In fact the association between a related kinase, Aurora A and a microtubule associated protein TPX2 can be used to draw comparisons with Aurora B:INCENP-IN-Box interactions. TPX2 is an allosteric activator of Aurora A kinase and this association also stimulates Aurora A activity in an ATP dependent manner (Eyers et al., 2003).

Aurora B kinase is regulated by other cofactors as well. Microtubules together with another inner centromeric protein telophase disc-60 (TD-60) activate Aurora B kinase in vitro (Rosasco-Nitcher et al., 2008). Moreover, partial loss of TD-60 impairs the ability of Aurora B kinase to auto-phosphorylate its T-loop and phosphorylate INCENP on Ser 850 (TSS) motif (Rosasco-Nitcher et al., 2008), which explains the requirement of TD-60 in full activation of the kinase. The mechanistic role of microtubules in activating Aurora B is less well characterized. 
CPC binds microtubules through two distinct domains. INCENP has a central coiled coil region that can bind microtubules (Mackay et al., 1998) and recombinant Aurora B bound to the IN-box can also bind microtubules (Rosasco-Nitcher et al., 2008). Phospho-regulation of microtubule interaction of CPC was first shown in S. cerevisiae. Cdk1 and Aurora B phosphorylates the CPC to prevent preanaphase spindle association and this inhibition is alleviated after anaphase onset to allow CPC to localize at the spindle midzone (Nakajima et al., 2011). Yeast Aurora B, Ipl-1, interacts with the yeast homologue of EB1, Bim1p, to localize to anaphase spindle and regulate the spindle microtubule dynamics (Zimniak et al., 2009). This interaction is also regulated by Cdk1 kinase Cdc28 by phosphorylating Ipl-1 near the Bim1p 'SXIP' interaction motif that inhibits preanaphase localization of Ipl-1/Aurora B to the spindle (Zimniak et al., 2012). These data suggests that microtubule association is tightly regulated and there exists multiple modes of inhibiting preanaphase CPC-microtubule association. That brings up the question as to how microtubules activate Aurora B in prometaphase, when it is most active. It is important to understand the interplay of CPC-microtubule interaction and activation of Aurora B kinase by microtubules.

Mps1, a dual specificity mitotic kinase, was first identified in S. cerevisiae where it was observed that a mutant allele had a defect in spindle pole body duplication that generated monopolar spindles (Winey et al., 1991) and had defective spindle checkpoint (Weiss and Winey, 1996). More recently it was shown that the human homologue had a similar role in the spindle checkpoint but cells depleted of Mps1 had little trouble in centrosome duplication (Stucke et al., 2002; Maciejowski et al., 2010). Mps1 is shown to work in concert with Aurora B kinase (Saurin et al., 2011; van der Waal et al., 2012). It 
phosphorylates Borealin to activate Aurora B and replacing Borealin with fourphosphosite mutant $\left(\right.$ Borealin $^{4 \mathrm{~A}}$ ) to perturb Mps1 phosphorylation leads to reduced centromeric activation of Aurora B and chromosome alignment defects in U2OS cells (Jelluma et al., 2008). Later it was shown that Mps1 regulates the dimerization state of Borealin by phosphorylating Thr230, one of the four sites identified in the earlier study, and mutating this residue to alanine phenocopies the 4A-mutant (Bourhis et al., 2009). Although there are interesting implications of Mps1 phosphorylating Borealin on CPC function, how it translates to regulation of Aurora B activity is not well understood.

CPC gets its name from the fact that it localizes to chromatin during early mitosis to finally reach the center of the cell (Earnshaw and Bernat, 1991). Yet little is known about the effect of DNA on Aurora B kinase activity. CPC localizes to chromatin in prophase and persists at the inner centromeres till anaphase onset. The CPC is detected initially along chromosome arms, but it progressively concentrates to the inner centromeres through prometaphase and metaphase (Ruchaud et al., 2007). Dasra A/B proteins are shown to play a critical role in loading CPC on chromatin and mediate chromatin-dependent activation of Aurora B kinase (Kelly et al., 2007). Depleting Dasra A in Xenopus egg extracts leads to failure of bipolar spindle formation (Sampath et al., 2004). Xenopus Dasra A is observed to bind non-centromeric chromatin beads dipped in Xenopus egg extracts by immunofluorescence (Sampath et al., 2004) but a distinct DNAbinding domain is not yet characterized. It will be important to analyze if DNA has any direct effect on regulating Aurora B activity. It is reasonable to hypothesize that DNA provides a platform to enrich CPC that allows auto-activation of the kinase to reach higher activity state. 


\section{Results}

Purification of the Chromosome passenger complex from E. coli

In order to perform biochemical characterization of the chromosomal passenger complex (CPC) we set out to purify the intact complex. We expressed full length INCENP, Dasra-A, Survivin and C-terminal 6-His tagged Aurora B from two bi-cistronic vectors by co-transforming BL21-pLysS E.coli strain (Fig. 3-1A). After affinity purification with Ni-NTA beads followed by size-exclusion chromatography we obtained the full CPC complex as shown by comigration of all four CPC proteins by Coomassie blue staining (Fig. 3-1B). The full complex, with a combined molecular weight of $228 \mathrm{kDa}$, eluted at a size larger than $670 \mathrm{kDa}$ (approx. 1.5 MDa) on a Superose 6 column. This suggests that the CPC is either a multimeric complex (possibly hexamer) or has an elongated shape that has an apparent stokes radius comparable to a 1.5 MDa globular protein. The native complex runs in similar fractions in Xenopus mitotic extracts where sedimentation analysis confirmed that the native complex is a highly elongated $\sim 250 \mathrm{kDa}$ complex consistent with one subunit of each protein (Bolton et al., 2002). We performed sedimentation analysis of purified CPC on a $5-30 \%$ sucrose gradient and found that the complex sedimented at 6.7S (Figure 3-1 B'). Siegel and Monty (Siegel and Monty, 1966) have derived equations using hydrodynamic measurements of molecules to overcome the contribution of shape and accurately measure the molecular weight. Similar calculations suggest that purified $\mathrm{CPC}$ is approximately $300 \mathrm{kDa}$. This suggests that it is also a monomer with one subunit of each protein.

We also purified the 'catalytic subunit' of the chromosome passenger complex that has N-terminal tagged full length Aurora B co-expressed with C-terminal INCENP 
fragment (IN-Box). We expressed two versions of IN-Box with residues 790-856 of INCENP (referred as $\mathrm{AI}^{790-856}$ ) or residues $790-847$ of INCENP (referred as $\mathrm{AI}^{790-847}$ ). We purified both GST- and 6-His tagged versions of this complex. Furthermore, we also purified both these complexes with a N-terminal GST-tagged truncated version of Aurora B (60-362) $-\Delta \mathrm{N} 60-\mathrm{AI}^{790-856}$ and $\Delta \mathrm{N} 60-\mathrm{AI}^{790-847}$ (Sessa et al., 2005). Fig. 3-1C shows a schematic of all the versions and vector maps are shown in appendix. Auto-phosphorylation by Aurora B regulates specific activity of the CPC

It has been shown that Aurora B mediated phosphorylation of critical resides in the INCENP IN-Box 'TSS' motif is necessary to stimulate its activity (Sessa et al., 2005). $\Delta \mathrm{N} 60-\mathrm{AI}^{790-856}$ had approximately 7 -fold more activity than $\Delta \mathrm{N} 60-\mathrm{AI}^{790-847}$ that lacks the 'TSS' motif. We compared kinase activity of $\mathrm{CPC}$ to $\mathrm{AI}^{790-856}$ at various concentrations and found that although at lower concentrations they were comparable, CPC shows a non-linear increase in activity at higher concentrations (Fig. 3-1D, 3-3F). We further compared CPC activity kinetics to both $\mathrm{AI}^{790-856}$ and $\mathrm{AI}^{790-847}$. We observed a similar nonlinear spike in CPC activity after an initial lag phase. This increase was absent in both forms of the catalytic domain (Fig. 3-1E). We envisioned that the full complex has a domain that is lacking in $\mathrm{AI}^{790-856}$ that allows auto-activation. We designed an assay to determine if CPC that has been inactivated by pretreatment with $\lambda$-phosphatase can be activated by a small amount of active CPC (Fig. 3-1F). We first pretreated a high concentration of CPC with $\lambda$ phosphatase (CPC - 4X(I)), which inactivated the kinase. We measured the activity of four-fold lower concentration of CPC that was not pretreated (CPC(1X)). We observed a 3.5 fold increase in activity when we mixed the $\lambda$ phosphatase treated 4X(I) kinase over 1X CPC (1X+4X(I)) (Fig. 3-1F'). In contrast, a 
similar assay with $\mathrm{AI}^{790-856}$ showed less than 2 fold change in $1 \mathrm{X}+4 \mathrm{X}(\mathrm{I})$ mix over $1 \mathrm{X}$ $\mathrm{AI}^{790-856}$ (Fig. 3-1G,G'), while that for $\mathrm{AI}^{790-847}$ failed to show any activation of the $1 \mathrm{X}$ with a change of less than 1.5 fold (Fig. $\left.3-1 \mathrm{H}, \mathrm{H}^{\prime}\right)$. We conclude that CPC has the ability to auto-activate Aurora B kinase activity in a concentration dependent manner and this requires a domain outside the catalytic subunit $\mathrm{AI}^{790-856}$. 


\section{Figure 3-1: Purification and auto-activation of CPC}

A) Plasmid map of pET28b-INCENP-AuroraB-6His and pET15b-Survivin-DasraA (No Tag). B) CPC was purified to homogeneity (highlighted fraction was collected for all assays). Fractions 7-18 from a Superose 6 FPLC run was TCA precipitated and run on a 15\%-polyacrylamide SDS-PAGE gel and stained with coomassie blue. Fractions are highlighted to show where Thyroglobulin $(670 \mathrm{kDa})$ and $\gamma$-globulin $(159 \mathrm{kDa})$ elutes on the same column to emphasize on effect of CPC's shape on eluting as a 1.5MDa protein even though its combined molecular weight is $228 \mathrm{kDa}$. B') Recombinant CPC sedimented on a $5-30 \%$ sucrose density gradient. CPC sediments at $6.7 \mathrm{~S}$. Western blot showing INCENP, Aurora B, phospho-Tloop (AurB) and phospho-S850(INCENP) staining of all fractions. Fraction from marker sedimented on a parallel gradient are highlighted - $1.96 \mathrm{~S}, 3.5 \mathrm{~S}, 6.7 \mathrm{~S}$ and $11.5 \mathrm{~S}$. C) Schematic representation of purified proteins. D) Comparison of in vitro kinase activity of $\mathrm{CPC}$ and $\mathrm{AI}^{790-856}$ titrated over the indicated range. Kinase activity is represented as pmol phosphate (PO4) incorporation on $1 \mu \mathrm{g}$ Histone-H3 tail-GST used as substrate. E) Kinetics of $10 \mathrm{nM}$ kinase $\mathrm{CPC}, \mathrm{AI}^{790-856}$ or $\mathrm{AI}^{790-847}$ was determined by comparing HistoneH3-tail-GST phosphorylation over 24 minutes with measuring pmol phosphate incorporated at indicated timepoints. F) Kinetics of $10 \mathrm{nM}$ active CPC (5X) was compared to $2 \mathrm{nM}$ active CPC (1X), 8nM Lambdaphosphatase treated inactive CPC (4X(I)) and a mixture of $2 \mathrm{nM}$ active and $8 \mathrm{nM}$ inactive CPC (1X+4X(I)) over 20 minutes by measuring phosphorylation of myelin basic protein (MBP). F') Activity of the $1 \mathrm{X}+4 \mathrm{X}(\mathrm{I}) \mathrm{CPC}$ mixture was plotted as a factor of $1 \mathrm{X} \mathrm{CPC}$ to determine the fold change and compared to $5 \mathrm{X}$ CPC. G,G') Kinetics and fold change in activity plotted for the same experiment explained in $\mathbf{F}$ but performed with $\mathrm{AI}^{790-856}$. $\mathbf{H}$ ) Kinase activity of $10 \mathrm{nM}$ active $\mathrm{AI}^{790-847}$ (5X) compared to $2 \mathrm{nM}$ active $\mathrm{AI}^{790-847}$ (1X), $8 \mathrm{nM}$ Lambda-phosphatase treated inactive $\mathrm{AI}^{790-847}(4 \mathrm{X}(\mathrm{I}))$ and a mixture of $2 \mathrm{nM}$ active and $8 \mathrm{nM}$ inactive $\mathrm{AI}^{790-847}(1 \mathrm{X}+4 \mathrm{X}(\mathrm{I}))$ at 20 minute timepoint as measured by pmol phosphate incorporated on MBP substrate. $\mathbf{H})$ Activity of the $1 \mathrm{X}+4 \mathrm{X}(\mathrm{I}) \mathrm{AI}^{790-847}$ mixture was plotted as a factor of $1 \mathrm{X} \mathrm{AI}^{790-847}$ to determine the fold change and compared to $5 \mathrm{X}$ $\mathrm{AI}^{790-847}$. 
A
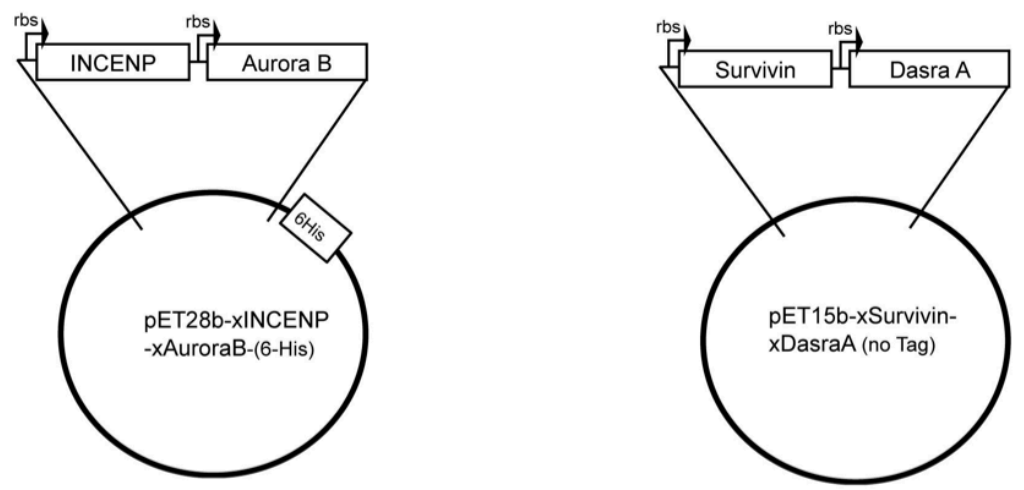

B

B'

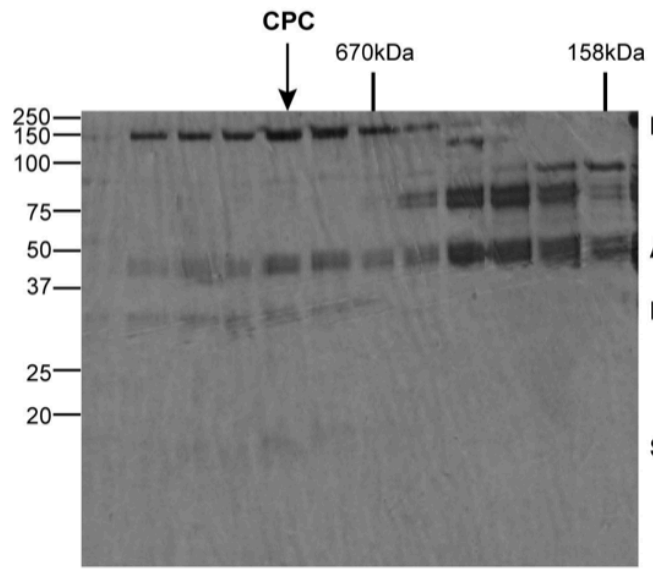

INCENP

Aurora B

Dasra A

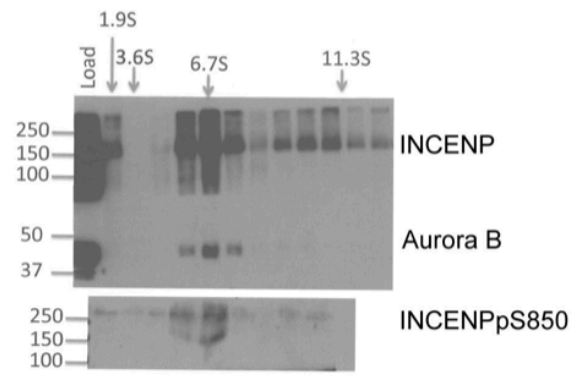

Survivin

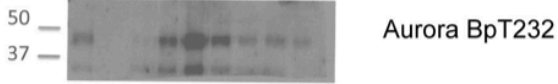

C

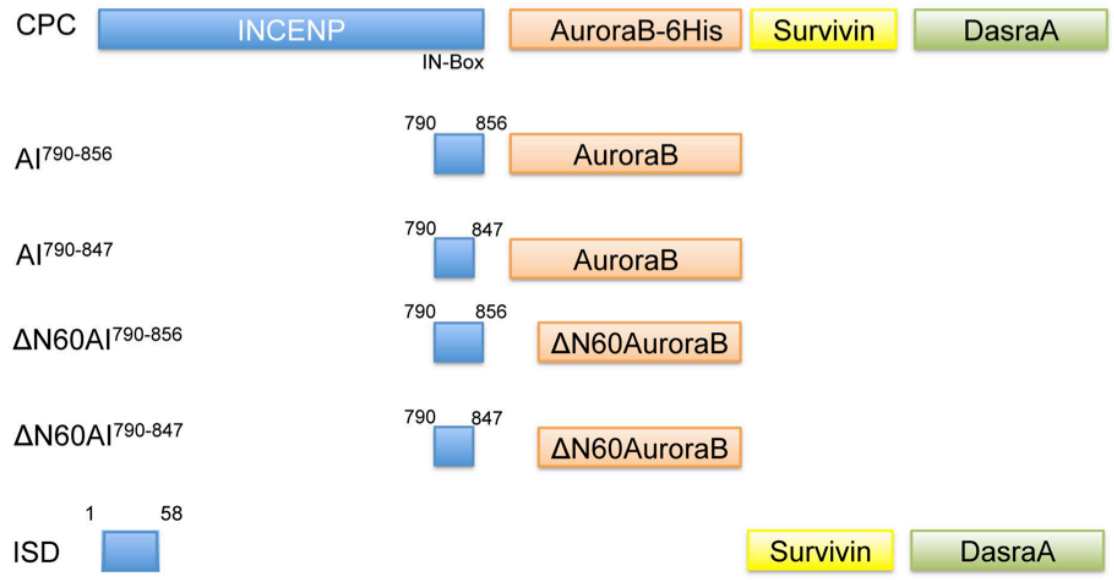

Figure 3-1 
D

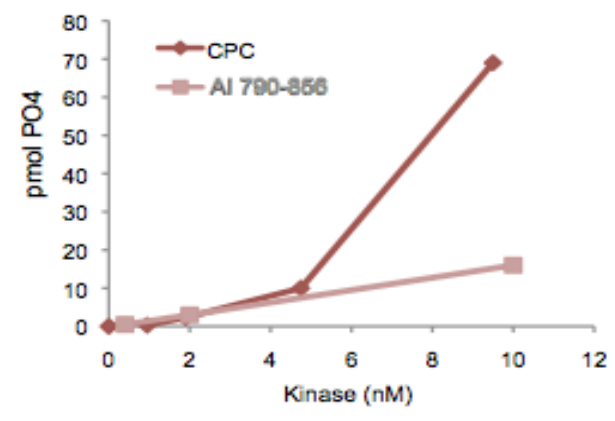

F

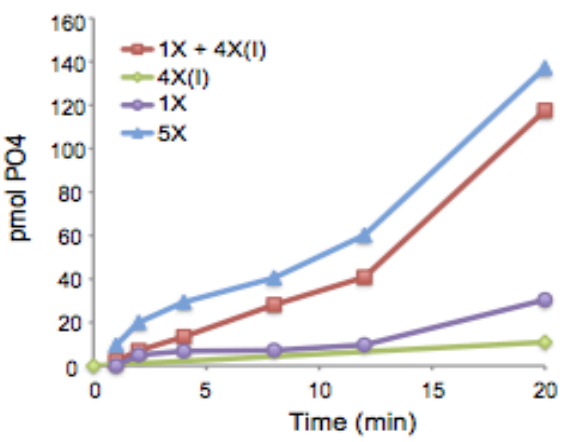

G

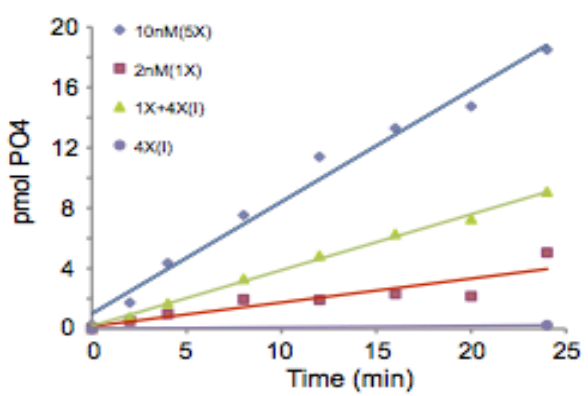

H

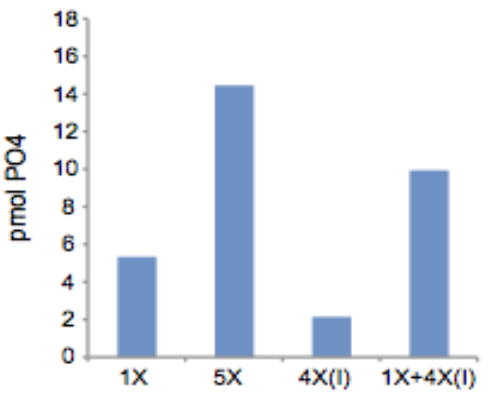

E

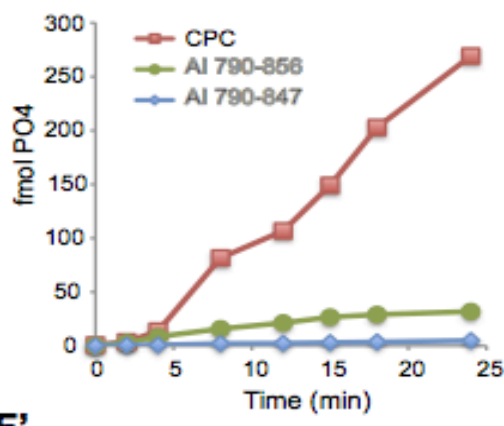

F'

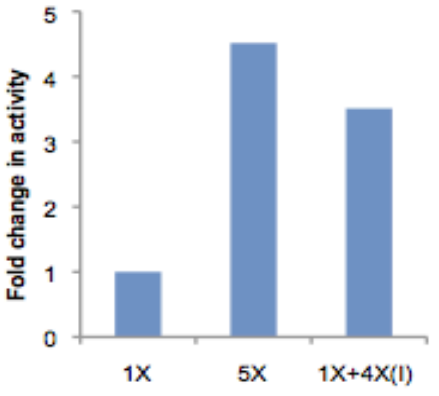

G'

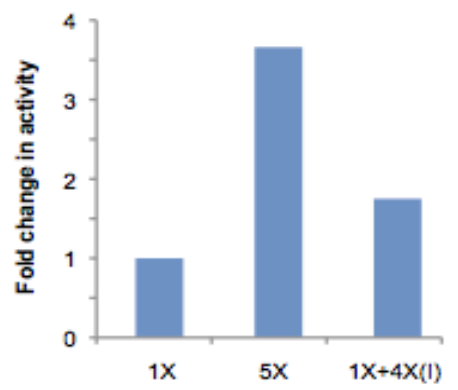

H'

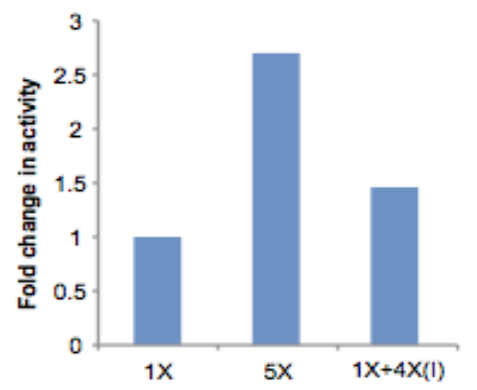

Figure 3-1 


\section{Cofactors that stimulate Aurora B activity}

\section{Monopolar spindle 1(Mps1)}

INCENP, TD-60 and microtubules are known cofactors of Aurora B kinase (Sessa et al., 2005; Rosasco-Nitcher et al., 2008). We tested additional candidates as cofactors that stimulate Aurora B activity in vitro. Mps1 stimulates Aurora B activity by phosphorylating Borealin in vivo (Jelluma et al., 2008). We immuno-precipitated Mps1 from Xenopus egg extract and measured whether MPS1 could activate CPC kinase that had been inactivated by $\lambda$-phosphatase treatment. Because there are two kinases we measured activity on two different substrates. Both kinases could phosphorylate myelin basic protein and the mixture of Mps1 and the $\lambda$-phosphatase treated CPC (5nM) was greater than either kinase alone. In fact the sum of the Mps 1 and $\lambda$-phosphatase treated CPC was comparable to the sum of the Mps1 and the CPC (5nM) before inactivation (Figure 2B). A similar trend was seen using histone $\mathrm{H} 3$ as a substrate, but this experiment was more informative because Histone $\mathrm{H} 3$ is a poor substrate for MPS1 allowing direct visualization of the stimulation of CPC (Fig. 3-2C). Although this is our initial interpretations, it is possible that Mps1 is stimulated by inactive CPC allosterically. This can be tested by repeating the same experiment with kinase dead CPC. Additionally, it is possible that Mps1 immuno-precipitated from CSF extracts is in complex with an unknown kinase that is responding to $\mathrm{CPC}$ addition.

We further investigated if the activation is mediated through similar mechanism involving Dasra A phosphorylation by Mps1. Comparing the Mps1 phosphorylation sites on Borealin to Dasra A we found two out of four residues, S112 and S181 of Dasra A, were conserved. We mutated these residues to alanines but that did not eliminate Dasra A 
phosphorylation by Mps1 (Fig. 3-2D). We conclude that MPS1 is an excellent activator of inactive CPC in vitro, but we have not identified the activating phosphorylation site.

\section{Centromere targeting sub-unit of CPC (ISD)}

Next we tested if association with the rest of the CPC is critical in activating the catalytic domain $\mathrm{AI}^{790-856}$. Since whole complex $\mathrm{CPC}$ had higher activity than $\mathrm{AI}^{790-856}$ it was reasonable to test if adding recombinant sub-complex ISD that comprised of full length Survivin, full length Dasra A and residues 1-58 of INCENP would stimulate kinase activity of Aurora B. We observed modest increase in Aurora B kinase activity after adding ISD (Fig. 3-2E)

\section{DNA and double strand breaks}

Aurora B is found on chromatin from prophase to metaphase (Ruchaud et al., 2007). Hence we asked if DNA stimulates Aurora B kinase activity. We tested CPC (10nM) kinase activity in the presence of short oligo (50fmol), circular plasmid DNA (20fmol), blunt cut (EcoR $V$ ) plasmid DNA (20fmol) and single digested plasmid DNA with sticky ends (20fmol). All forms of DNA with the exception of short oligo showed more than 2 fold stimulation in kinase activity (Fig. 2F). We titrated a circular plasmid containing a repetitive $5 \mathrm{~S}$-gene sequence to further validate this result. We observed a sharp increase in kinase activity in the presence of lower concentrations (10-50fmol) of plasmid, which flattened out, and finally this stimulation was lost on adding more than 100fmol of plasmid (Fig. 2G). One explanation for these concentration dependent effects is that DNA concentrates the CPC, which initiates the auto-activation cycle. Thus lower 
concentrations of DNA stimulate the CPC better because multiple CPC molecules are bound to the same plasmid, which at higher concentrations are segregated to separate plasmid molecules. We have included $5 \mathrm{nM}$ CPC in this reaction and we observe the inhibitory effect above $200 \mathrm{fmol}$ of plasmid. To test if indeed DNA facilitates the autoactivation process, we performed our activation assay by adding $2 \mathrm{nM}$ active CPC (1X) to $8 \mathrm{nM} \lambda$-phosphatase treated CPC $(4 \mathrm{X}(\mathrm{I}))$ in the presence or absence of DNA. $1 \mathrm{X}+4 \mathrm{X}(\mathrm{I})$ mix had more Aurora B activity in the presence of DNA than in its absence (Fig. 3-2H) suggesting that DNA facilitates CPC auto-activation.

\section{Microtubules}

Microtubules were shown to activate CPC in the presence of TD-60 (RosascoNitcher et al., 2008) and adding microtubules to CPC stimulates its kinase activity (Fig. 3-2I,J). With these data we wanted to determine if the presence of microtubules stimulated the $1 \mathrm{X}+4 \mathrm{X}(\mathrm{I})$ activation reaction. Indeed we observed that $1 \mathrm{X}+4 \mathrm{X}(\mathrm{I}) \mathrm{mix}$ had more activity after adding microtubules than in its absence (Fig. 3-2I,J). We speculate that microtubules provide a platform for $\mathrm{CPC}$ to concentrate and auto-activate but it is also possible that microtubules have an allosteric effect on the kinase. We will revisit this hypothesis later in the chapter. 


\section{Figure 3-2: Cofactors regulating CPC activity/auto-activation.}

A) Mps 1 immuno-precipitated from CSF arrested Xenopus egg extract was added to 5nM $\lambda$-phosphatase treated inactive CPC to assay its ability to activate Aurora B kinase. Autoradiograph and coomassie stained gel showing $\gamma^{32} \mathrm{P}$-labeled phosphate incorporation on HistoneH3-tail and MBP. B) Quantification of pmol phosphate incorporation on MBP by active CPC, Mps1, inactive CPC(I) and a mixture of Mps1+CPC(I). C) Quantification of pmol phosphate incorporation on HistoneH3-tail by active CPC, Mps1, inactive CPC(I) and a mixture of Mps1+CPC(I). Both $\mathrm{CPC}$ and Mps1 phosphorylate MBP, while HistoneH3-tail is a preferred substrate for CPC. The mixture of Mps1 and CPC(I) has more activity than the sum of their individual activities depicting CPC(I) activation by Mps1. D) Mps1 immuno-precipitated from CSF arrested Xenopus egg extract was used to check phosphorylation of wildtype Xenopus Dasra A and a mutant with S112A, S181A changes. SS-AA mutant xDasra A was still phosphorylated by Mps1. E) Adding recombinant ISD $(800 \mathrm{nM})$ to $10 \mathrm{nM} \mathrm{AI}^{790-856}$ stimulates its kinase activity. Activity was measured as pmol phosphate incorporation on MBP. F) Various forms of DNA fragments were added to $10 \mathrm{nM} \mathrm{CPC}$ and activity was assayed for X minutes. Activity is measured as pmol phosphate incorporated on MBP. (Oligo - 18 nucleotide long - 50fmol; cirDNA - 5kb plasmid - 20fmol; blunt - 5kb plasmid digested with EcoRV - 20fmol; sticky $5 \mathrm{~kb}$ plasmid digested with BamHI - 20fmol) G) A 5kb plasmid DNA was titrated into $5 \mathrm{nMnM}$ CPC over the indicated range and kinase activity was measured as pmol phosphate incorporated on MBP. H) Activation of $8 \mathrm{nM}$ lambda-phosphatase treated inactive CPC by $2 \mathrm{nM}$ active CPC was assayed with or without adding $100 \mathrm{ng} 5 \mathrm{~kb}$ plasmid DNA. Kinetics of CPC activity was assayed over 40 minutes and measured by phosphorylation of MBP. I) Adding microtubules to CPC stimulates its kinase activity. Auto-radiograph and coomassie stained gel showing phosphorylation of MBP in the indicated conditions. J) Adding microtubules stimulates auto-activation reaction in $1 \mathrm{X}+4 \mathrm{X}(\mathrm{I})$ mixture. Kinase activity measured after 10 minutes by pmol phosphate incorporation on MBP. 
A

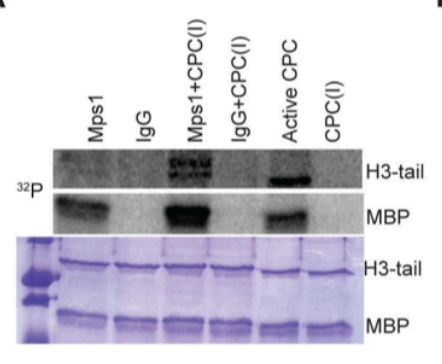

D

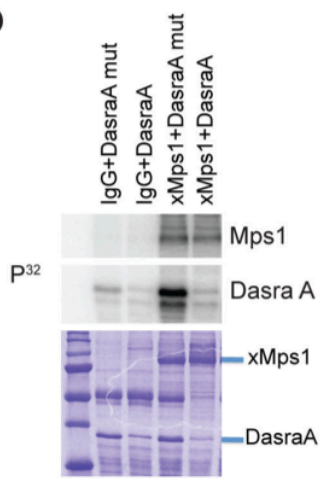

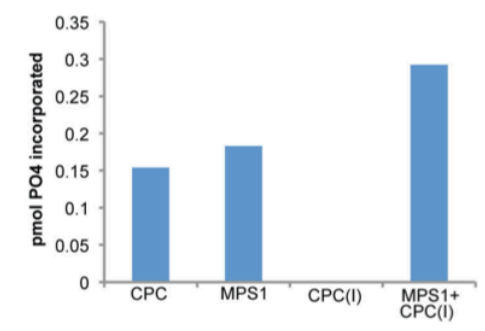

E
C

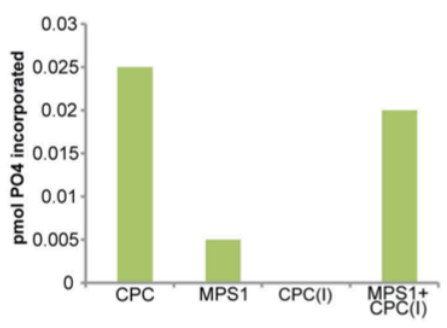

F

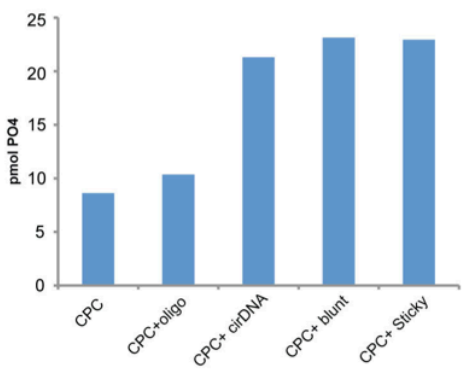

I
G

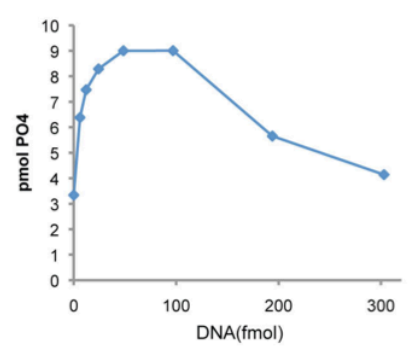

J

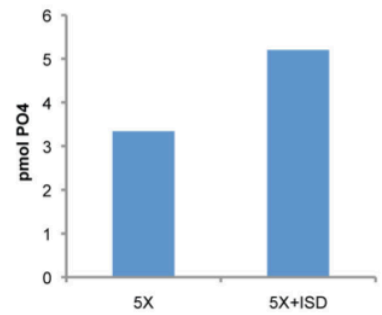

H
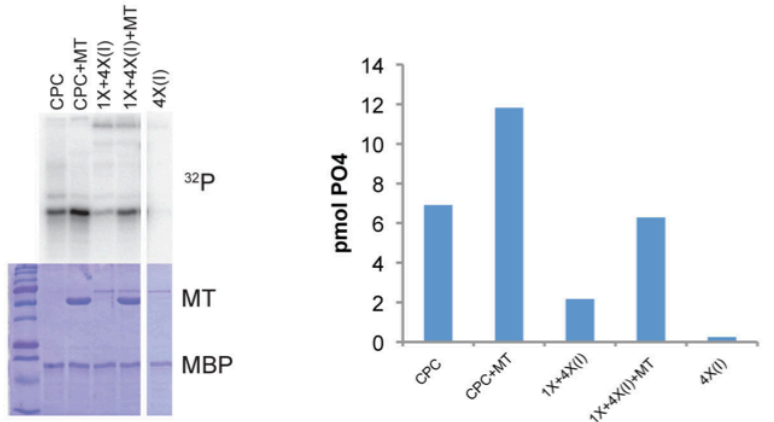

Figure 3-2 
Autophosphorylation of Aurora B's N-terminus positively and negatively regulates kinase activity.

A defining feature of the Aurora family of kinases are a domain of $~ 100$ amino acids on the $\mathrm{N}$-terminus and a $\mathrm{C}$-terminal extension of $\sim 20$ amino acids. The $\mathrm{N}$-terminal region of Aurora B is highly divergent in vertebrates (Fig. 3-4). This domain is also divergent between the mammalian Aurora kinases A, B and C. The role of the Nterminus is poorly understood although there are some reports that the N-terminus interacts with different cofactors to designate unique functions during mitosis (Tsai et al., 2003; Honda et al., 2003; Adams et al., 2000; Eyers et al., 2003; Sessa et al., 2005). In this section we will highlight a new role of N-terminus (1-60 residues) of Xenopus Aurora B as a modulator of kinase activity.

We compared the kinase activity of titrated $\mathrm{AI}^{790-856}$ with $\Delta \mathrm{N} 60-\mathrm{AI}^{790-856}$ and $\Delta \mathrm{N} 60-\mathrm{AI}^{790-847}$ and observed that the activity of the full-length kinase was significantly higher than the N-terminal deleted versions (Fig. 3-3A). This has not been previously reported. Since this effect was observed in an in vitro kinase assay we reasoned that this is an auto-phosphorylation mediated regulation. So we made point mutants of two threonine residues (Thr18 and Thr56) within the N-terminal region that partially matched the putative aurora B consensus motif for substrate phosphorylation (Cheeseman et al., 2002). Mutating threonine 56 to alanine (T56A) resulted in a reduction in kinase activity of the $\mathrm{AI}^{790-856}$ (Fig. 3-3B), which basically mimicked the effect of deleting the Nterminal 60 residues. Hence, we conclude that auto-phosphorylation of Aurora B Thr56 has a stimulatory effect on kinase activity. 
Mutating threonine 18 had quite the opposite effect. We observed that a T18A mutant form of $\mathrm{AI}^{790-856}$ had higher kinase activity than wildtype (Fig. 3-3E (w/o PI), F). This made us think of threonine 18 as an auto-inhibitory switch. To test if Aurora B can auto-phosphorylate itself to reduce its kinase activity we designed an assay in which we pre-incubated the kinase with ATP/ $\mathrm{Mg}^{2+}$ for 15 minutes before adding $\gamma_{-}{ }^{32} \mathrm{P}$ labeled ATP and substrate to the reaction to get a read out of activity. The pre-incubation step would allow auto-phosphorylation of Aurora B and then we can compare substrate phosphorylation of this pre-treated $\mathrm{AI}^{790-856}$ to $\mathrm{AI}^{790-856}$ that has not undergone such a pretreatment. Kinase activity was measured on two substrates and both showed reduced phosphorylation by pre-treated $\mathrm{AI}^{790-856}$ than untreated $\mathrm{AI}^{790-856}$, suggesting that Aurora $\mathrm{B}$ can inhibit its kinase activity by autophosphorylation (Fig. 3-3C,D). Then we performed the same assay with the T18A mutant form of $\mathrm{AI}^{790-856}$ and compared it to wildtype (Fig. 3-3E). As mentioned earlier T18A mutant had higher kinase activity than wildtype $\mathrm{AI}^{790-}$ ${ }^{856}$ and we observed minimal reduction in kinase activity after the pre-incubating T18A$\mathrm{AI}^{790-856}$ in $\mathrm{ATP} / \mathrm{Mg}^{2+}$, suggesting that phosphorylation of threonine 18 reduces Aurora $\mathrm{B}$ activity. Moreover, we compared the kinase activity of T18A-AI ${ }^{790-856}$ to $\mathrm{CPC}$ and $\mathrm{AI}^{790-}$ ${ }^{856}$ and observed that mutating Thr18 to alanine made $\mathrm{AI}^{790-856}$ a more active kinase than $\mathrm{AI}^{790-856}$ but it was still not as active as $\mathrm{CPC}$ (Fig. 3-3F). We conclude that the autophosphorylation of the N-terminus of Xenopus Aurora B can modulate kinase activity in vitro. 
Figure 3-3: N-terminal tail auto-regulates Aurora B kinase.

A) N-terminal (60 resides) tail of Aurora B is critical for full activation of kinase. Graph shows comparison of kinase activity of titrated amounts of $\mathrm{AI}^{790-856}, \Delta \mathrm{N} 60-\mathrm{AI}^{790-856}$ and $\Delta \mathrm{N} 60-\mathrm{AI}^{790-847}$. Activity was assayed by incubating MBP with indicated kinase for 10 minutes and measuring pmol phosphate incorporation. Red rectangle indicates activity of same amount of kinase. B) Single point mutant in N-terminal tail of Aurora B (T56A) has similarly reduced kinase activity as the $\mathrm{N}$-terminal truncated kinase $(\Delta \mathrm{N} 60)$ compared to wildtype kinase. Activity was assayed for 10nM kinase and measured by pmol phosphate incorporation on MBP. C \& D) Inhibitory auto-phosphorylation of Aurora B kinase observed after 15 minute pre-incubation in $\mathrm{ATP} / \mathrm{Mg}^{2+}$. $\mathrm{AI}^{790-856}$ activity assayed over a range of concentrations on MBP (C) and HistoneH3-tail (D) after incubating the kinase with ATP $/ \mathrm{Mg}^{2+}$. E) Single point mutant (T18A) in the N-terminal tail of Aurora B shows minimal auto-inhibitory effect of $\mathrm{ATP} / \mathrm{Mg}^{2+}$ preincubation. Kinase activity of $\mathrm{AI}^{790-856}$ (WT) was compared to the point mutant expressed as T18A-AI ${ }^{790-856}$ (T18A) with or without the ATP $/ \mathrm{Mg}^{2+}$ pre-incubation and measured as pmol phosphate incorporation on MBP. F) Comparing kinase activity of titrated concentrations of CPC, T18A-AI ${ }^{790-856}$ (T18A) and $\mathrm{AI}^{790-856}$ shows $\mathrm{T} 18 \mathrm{~A}$ to have a higher activity than wildtype form but less than CPC. Activity assayed over 2 minutes was measured as pmol phosphate incorporation on MBP. 
A

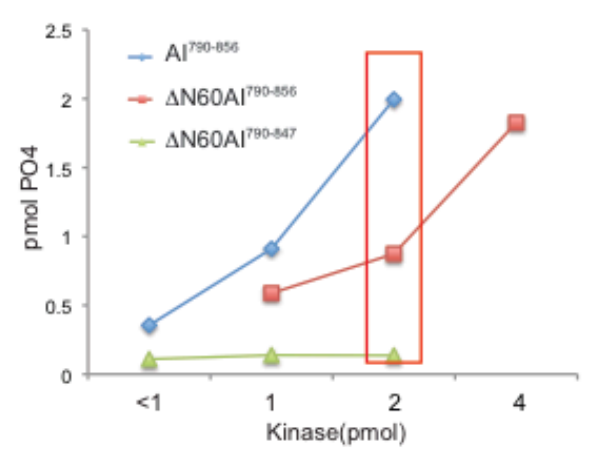

C

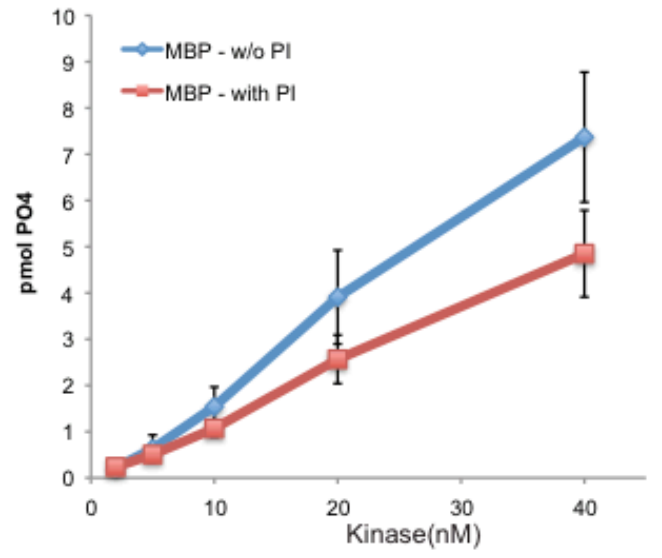

E

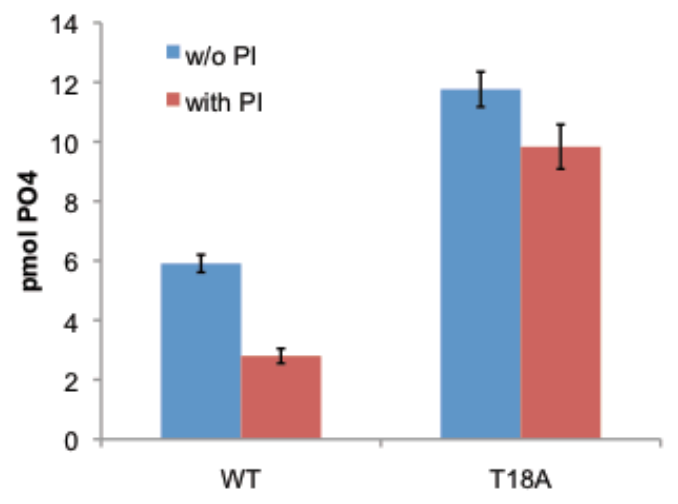

B

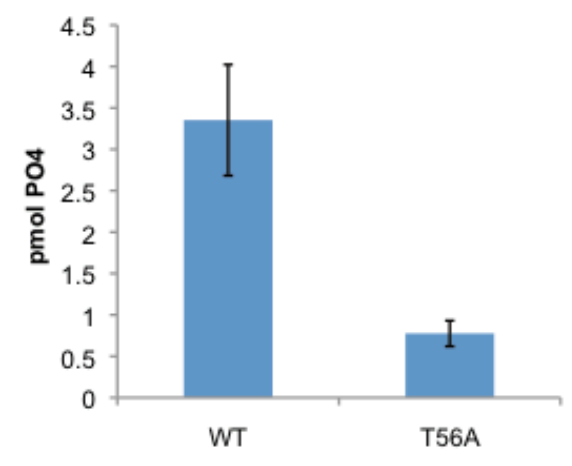

D

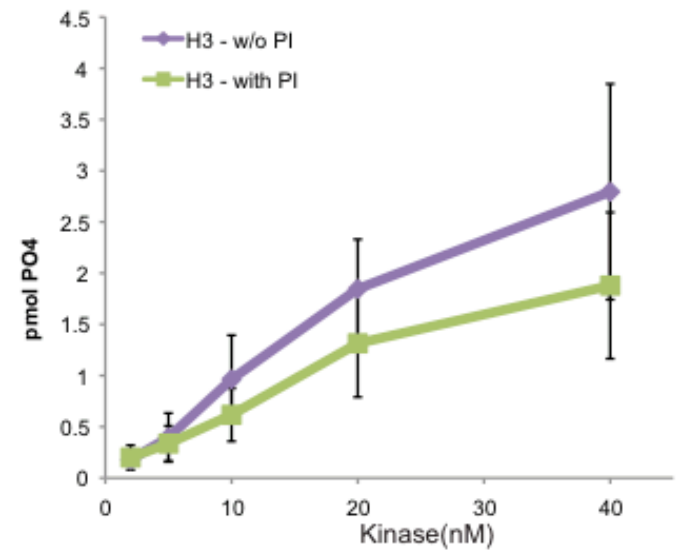

F

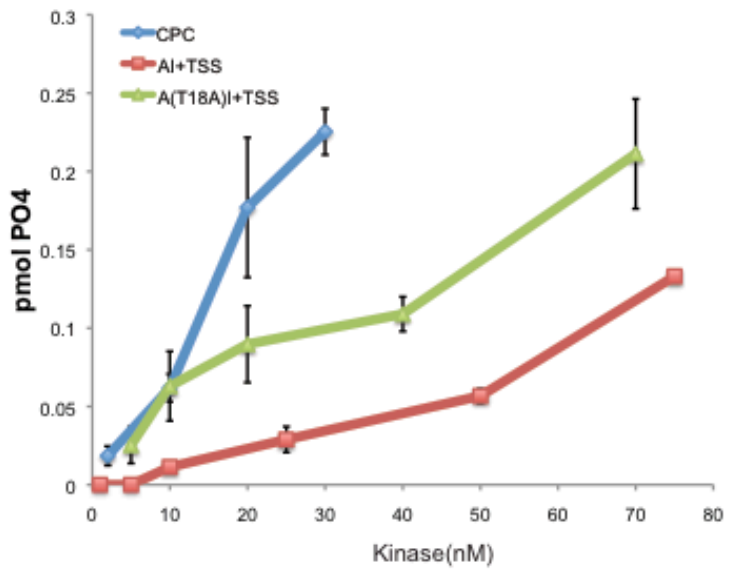

Figure 3-3 


\begin{abstract}
XI Aurora B - MEYKENLNPS--- XI AUTORA MERAVKENHKPSNVKVFHPMTEGPKRIPVSQPPSTQVRPPVTGVSAQRILGPSNVPQRVM me---KEN--PSnvkvf hpmtegpkripvsq--ts----P-sa--AQRvL-psnvpqrvm

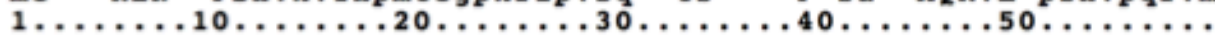

xl Aurora B ---KEPYVSTFTTPSDNLLAQRTQLSRITP----------SASSSVP--GRVAVSTEM xl AuroraA MQAQKPVLSNQKPTAQGLLRPATHGHQTSKPQGPNENRNPQQTSHSSTPNMEKKGSTDQG mqa--P-vS--------LL---T-----t-pqgpnenrnpqqs--SS-Pnm-r-a-s---

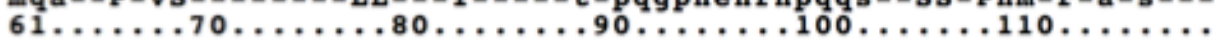

xI Aurora B PSQNTALAEMPKRKFTIDDFDIGRPLGKGKFGNVYLAREKQNKFIMALKVLFKSQLEKEG xl Aurora A KTLAVPKEEGKKKQWCLEDFEIGRPLGKGKFGNVYLARERESKFILALKVLFKSQLEKAG -s-----E--Kr-f-idDFdIGRPLGKGKFGNVYLAREK--KFIMALKVLFKSQLEK-G $121 \ldots \ldots 130 \ldots \ldots \ldots 140 \ldots \ldots \ldots 150 \ldots \ldots \ldots 160 \ldots \ldots 170 \ldots \ldots \ldots$

xI Aurora B VEHQLRREIEIQSHLRHPNILRMYNYFHDRKRIYLMLEFAPRGELYKELQKHGRFDEQRS xI Aurora A VEHQLRREVEIQSHLRHPNILRLYGYFHDACRVYLILDYAPGGELFRELQKCTRFDDQRS VEHQLRRE IEIQSHLRHPNILRMY-YFHD--RIYLmL E AP-GELYKELQK--RFDEQRS $181 \ldots \ldots 190 \ldots \ldots 200 \ldots \ldots 210 \ldots \ldots 220 \ldots \ldots 230 \ldots \ldots \ldots$

xl Aurora B ATPMEELADALHYCHERKVIHRDIKPENLLMGYKGELKIADFGWSVHAPSLRRRTMCGTL $x \mid$ Aurora A ALYIKQLAEALLYCHSKKVIHRDIKPENLLLGSNGELKIADFGWSVHAPSSRRTTLCGTL A-fm--LAdAL-YCH-TKVIHRDIKPENLLMG--GELKIADFGWSVHAPS -RR-TmCGTL

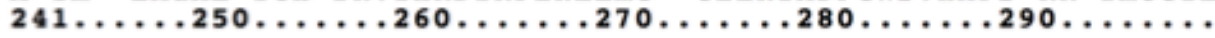

xl Aurora B DYLPPEMIEGKTHDEKVDLWCAGVLCYEFLVGMPPFDSPSHTETHRRIVNVDLKFPPFLS xl Aurora A DYLPPEMIEGRMHDEKVDLWSLGVLCYEFLVGKPPFETDTHQETYRRISKVEFQYPPYVS DYLPPEMIEGK-HDEKVDLW--GVLCYEFLVG-PPFds-sH-ET-RRI--Vd--fPPf IS

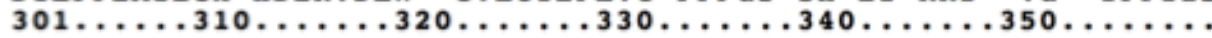

xI Aurora B DGSKDLISKLLRYHPPQRLPLKGVMEHPWVKANSRRVLPPVYQSTQSK xI Aurora A EEAKDLVSKLLKHNPNHRLPLKGVLEHPWIVKNSQQPKKKDEPLAGAQ d--KDLISKLLY--P--RLPLKGVMEHPWV--NS-----------

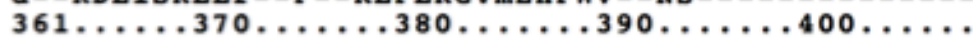

Figure 3-4: Alignment showing divergence in N-terminal domain of Xenopus Aurora kinases.

Alignment of Xenopus Auorora A and B showing conserved residues in green and similar residues in cyan boxes. Unique regions are highlighted in yellow and unmatched resides are not highlighted. Residues are numbers to match the Aurora A sequence. Alignment was performed using ClustalW in Biology Workbench 3.2. 


\section{CPC-microtubule interaction and auto-regulation by kinase activity}

INCENP binds tubulin, which is thought to mediate the CPC localization to the spindle midzone (Wheatley et al., 2001b; Earnshaw and Cooke, 1991). Purified CPC interacted with taxol stabilized microtubules with a $\mathrm{K}_{\mathrm{d}}$ of $\sim 1.5 \mathrm{uM}$ tubulin as assayed by sedimentation through a sucrose cushion (Fig. 3-5A,B). We visualized the CPC and a mixture of CPC and microtubules by negative stained electron microscopy (Fig. 3-5C). We detected elongated electron dense particles on grids that had CPC but not on control grids. We measured length of densities that appeared to be single CPC units and found a bimodal distribution of observed lengths (Fig. 3-5D). This suggested that CPC might exist at two conformations with the longest axes measuring $45 \mathrm{~nm}$ and $60 \mathrm{~nm}$ respectively. In the same experiment we also observed that these single densities clustered to form oligomerized structures. These structures had a distinct pattern of clustering into rosettes (not shown) and accounted for about half of the total CPC units.

We also performed electron microscopy of CPC-microtubule mixture. We observed coating of electron densities in patches on microtubules with CPC, which were absent on microtubules alone (Fig. 3-5E). There were two major kinds of densities on microtubules. Some that wrapped around microtubules in small stretches and the other were dense patches protruding out at fixed angles (45-60deg). This suggested that varying degree of CPC oligomerization could occur on microtubules and single CPC units could bind a microtubule through multiple binding sites to wrap around the polymer.

To investigate if Aurora B can bind microtubules directly in the absence of the INCENP coiled-coil binding site (Mackay et al., 1998), we performed a microtubule- 
pelleting assay with $\mathrm{AI}^{790-856}$. Above $2 \mathrm{uM}$ tubulin about $10 \%$ of the $\mathrm{AI}^{790-856}$ pelleted with tubulin and this did not increase beyond $20 \%$ with higher concentrations of microtubules (Fig. 3-5F,G). Since Aurora B can phosphorylate its own T-loop, AI ${ }^{790-856}$ purified from bacteria is a heterogenous mixture of differentially phosphorylated complex. We hypothesized that the heterogeneity of phosphorylation states generated populations of $\mathrm{AI}^{790-856}$ that bound tubulin with varying affinities. To generate a homogenous population of $\mathrm{AI}^{790-856}$ we performed two pre-treatments. First, we treated the $\mathrm{AI}^{790-856}$ with $\lambda$-phosphatase to de-phosphorylate the complex. Second, we incubated the $\mathrm{AI}^{790-856}$ with $\mathrm{ATP} / \mathrm{Mg}^{2+}$ to generate a fully phosphorylated population. We found that inactive $\mathrm{AI}^{790-856}$ ( $\left.\mathrm{AI}^{790-856}+\mathrm{L}-\mathrm{phos}\right)$ bound taxol-stabilized microtubules with higher affinity than untreated $\mathrm{AI}^{790-856}$ and that we could get almost $45 \%$ binding at high tubulin concentrations. In contrast, the affinity of fully phosphorylated $\mathrm{AI}^{790-856}\left(\mathrm{AI}^{790-856}+\mathrm{ATP}-\right.$ PI) was further reduced (Fig. 3-5F,G). This demonstrates that $\mathrm{AI}^{790-856}$ can bind microtubules and this affinity is decreased by auto-phosphorylation of $\mathrm{AI}^{790-856}$.

We further tested this by performing microtubule binding assay with various mutants of $\mathrm{AI}^{790-856}$ that are either catalytically inactive or has severely reduced kinase activity as compared to wildtype. A kinase dead K122R mutant showed very high affinity for microtubules as compared to $\mathrm{ATP} / \mathrm{Mg}^{2+}$ pre-treated $\mathrm{AI}^{790-856}$, similar to $\lambda$-phosphatase treated $\mathrm{AI}^{790-856}$. Since phosphorylation of the 'TSS' motif of INCENP IN-Box is also critical to full activation of Aurora B, we performed binding assays with point mutants of each residues of the 'TSS' motif. The T848A mutant has slightly higher kinase activity as compared to $\mathrm{S} 849 \mathrm{~A}$ and $\mathrm{S} 850 \mathrm{~A}$ mutants of $\mathrm{AI}^{790-856}$ and that reflected inversely on respective binding affinities for each mutant, with T848A mutant having the least affinity 
for microtubules amongst the three point mutants (Fig. 3-6). We conclude that the affinity of catalytic subunit of CPC for microtubule binding can be negatively regulated by autophosphorylation. 
Figure 3-5: Microtubule interaction and auto-regulation by catalytic domain of CPC.

A) Binding assay demonstrating CPC pellets with taxol-stabilized microtubules. 30nM CPC was incubated with indicated concentrations of taxol stabilized microtubules for 10 minutes and pelleted. Western blot of supernatant and pellets for each reaction shows INCENP and Dasra A in the pellet. B) Densitometric analysis of INCENP in the pellet plotted as a percentage of INCENP in 'load' showed CPC bound with microtubules with an approximate $\mathrm{K}_{\mathrm{d}}$ of $1.5 \mu \mathrm{M}$. C) Electron micrographs of negative stained (uranyl acetate) CPC sprayed on formvar grids. Each figure is a representative image of a single CPC unit. D) Histograms showing frequency distribution of measured (long axis) lengths of CPC units in angstroms. E) Electron microscopy reveals CPC binds microtubules in scattered patches. Electron micrographs of negative stained (uranyl acetate) microtubules compared to mixture of microtubule $(3 \mu \mathrm{M})$ and $\mathrm{CPC}(30 \mathrm{nM})$. F) Binding assay demonstrating catalytic subunit of $\mathrm{CPC}\left(\mathrm{AI}^{790-856}\right)$ binds microtubules and this binding is negatively regulated by Aurora B dependent phosphorylation. $\mathrm{AI}^{790-856}$ was treated with Lambda-phosphatase to remove all phosphorylation marks on $\mathrm{AI}^{790-856}\left(\mathrm{AI}^{790-856}+\mathrm{L}-\mathrm{Phos}\right)$ or pre-incubated with $\mathrm{ATP} / \mathrm{Mg}^{2+}$ to allow auto-phosphorylation of the complex $\left(\mathrm{AI}^{790-}\right.$ ${ }^{856}+$ ATP-PI). These pretreated $\mathrm{AI}^{790-856}$ were then incubated with indicated concentrations of taxol-stabilized microtubules. Western blot of supernatant and pellet at indicated conditions shows Aurora B in pellets. G) Densitometric analysis of Aurora B in the pellet plotted as a percentage of Aurora B in 'Load' shows variable affinities of differently treated $\mathrm{AI}^{790-856}$. 
A

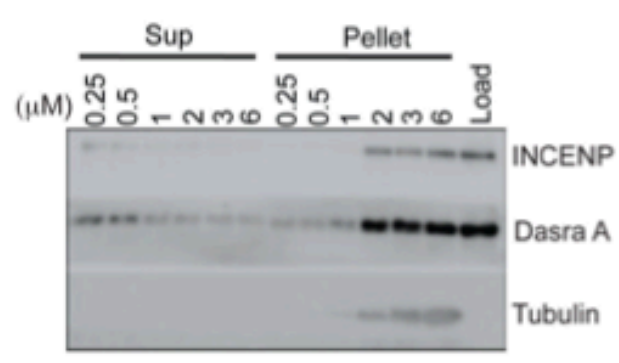

C
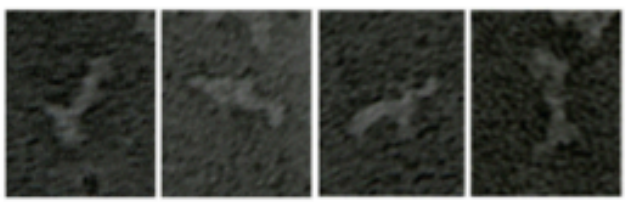

E
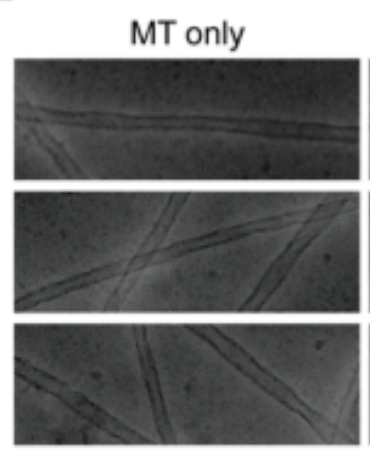

B

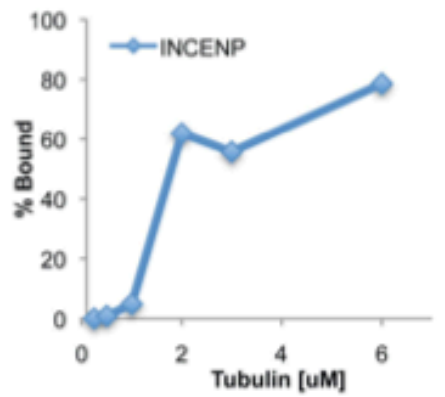

D

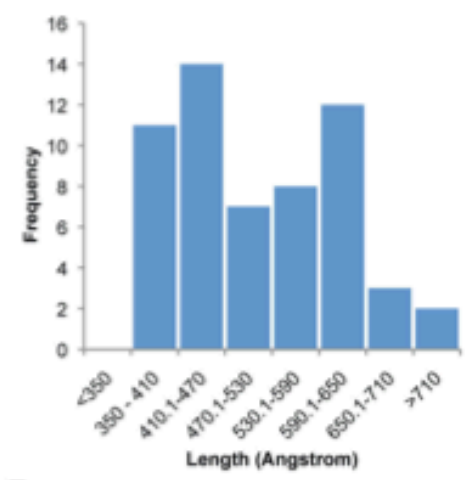

F
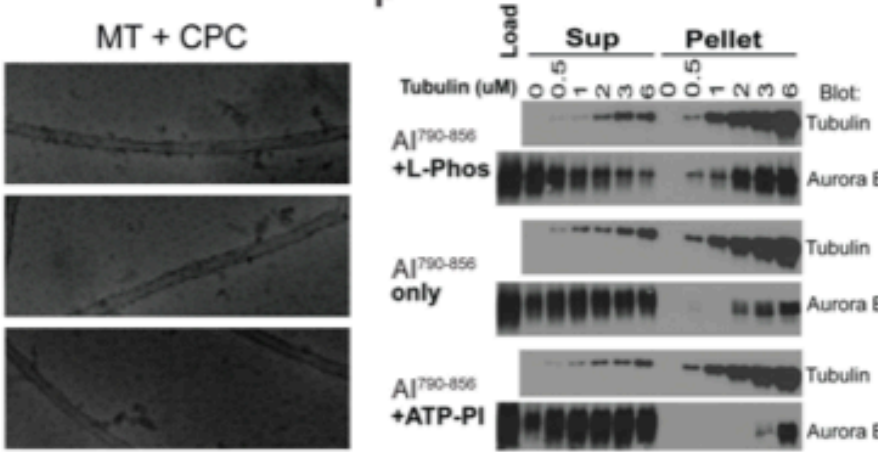
Alposese $\quad \cdots+-\infty-\infty$ Tubulin

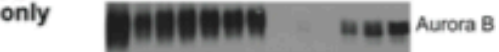

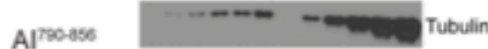
+ATP-PI 100 Aurora B

\section{G}

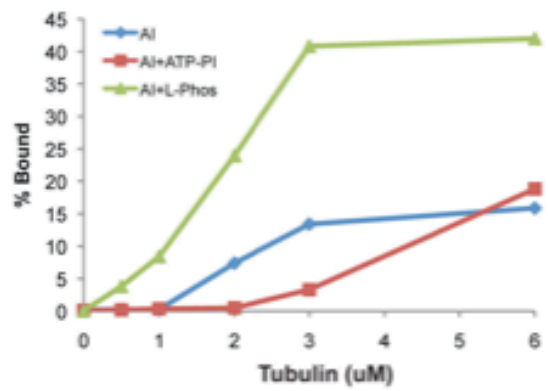

Figure 3-5 


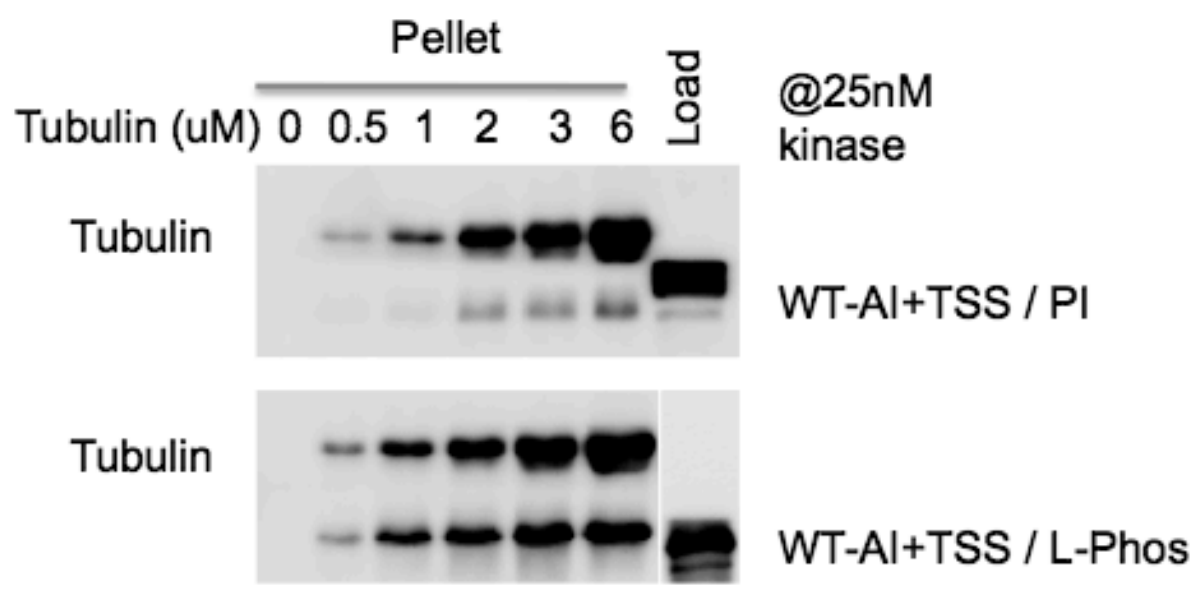

Tubulin

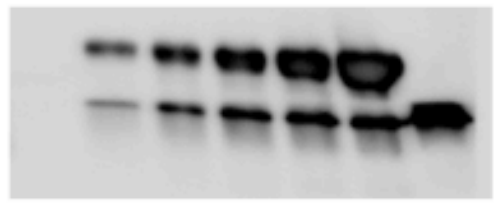

$\mathrm{A}(\mathrm{K} 122 \mathrm{R}) \mid+\mathrm{TSS} / \mathrm{PI}$

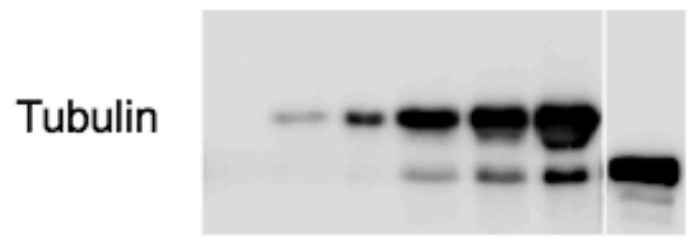

$A l+\left(A^{848}\right) S S / P I$

Tubulin

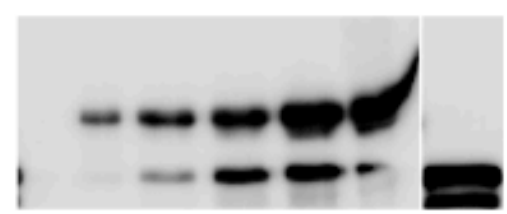

$\mathrm{Al}+\mathrm{TS}\left(\mathrm{A}^{850}\right) / \mathrm{PI}$

Figure 3-6: Microtubule binding assay with $A I^{790-856}$ mutants shows inverse relationship between kinase activity and microtubule affinity.

Western blot of tubulin (anti-tubulin, Dm1 $\alpha$ ) and Aurora B (Rb316) of pellet samples at indicated tubulin concentrations. Load depicts $100 \%$ of $\mathrm{AI}^{790-856}$ mutant mixed with each concentration of taxol-stabilized microtubules. 


\section{Discussion}

CPC performs multiple tasks in mitosis. It integrates signals from various sources like the kinetochores, centromeric chromatin and the spindle to recognize errors and then phosphorylates particular substrates to correct those errors. It is difficult to study the exact contribution of each signal in isolation in vivo. To understand the mechanism of regulation of CPC by such signals through numerous cofactors, including auto-regulation within the complex, we decided to purify recombinant Xenopus CPC.

There is evidence of signal amplification in biological processes, for example Gprotein coupled receptor mediated signal transduction and protein-kinase cascade. Aurora $\mathrm{B}$ enrichment at the centromeres during prometaphase is essential in generating error free chromosome segregation. It is suggested that this enrichment amplifies the Aurora B activity state and creates a zone of high kinase activity centered at the inner centromeres. To determine if there is any concentration dependence in regulating Aurora B activity state we assayed kinase activity of titrated amounts of CPC. Simultaneously we wanted to know if Aurora B activity was changed when in complex with the rest of the chromosomal passenger proteins. We compared the kinase activity of CPC with its catalytic subunit $\mathrm{AI}^{790-856}$, which is Aurora $\mathrm{B}$ in complex with a highly conserved Cterminal INCENP fragment IN-Box. We observed 7-fold stimulation in CPC activity at concentrations above $5 \mathrm{nM}$ and this was absent in $\mathrm{AI}^{790-856}$ (Fig. 3-1D). This suggested that $\mathrm{CPC}$ could initiate an auto-activation process above a threshold concentration. We further tested this hypothesis by designing the activation assay in which we mixed small amounts of active CPC to a large pool of $\lambda$-phosphatase inactivated CPC. If the assay was done at $10 \mathrm{nM}$ total $\mathrm{CPC}$ concentration $(1 \mathrm{X}+4 \mathrm{X}(\mathrm{I}))$ we observed that the small active pool 
was able to activate the larger inactive pool (Fig. 3-1F). When we performed this at concentrations below $5 \mathrm{nM} \mathrm{CPC}$ we did not observe this stimulation. This confirms that CPC auto-activates Aurora B kinase activity above in a concentration dependent manner. Then we asked if the regulatory subunits of CPC - INCENP, Dasra A or survivin are required for this auto-activation. Both versions of the minimal catalytic subunit, $\mathrm{AI}^{790-847}$ lacking the 'TSS' motif of IN-Box or $\mathrm{AI}^{790-856}$ that has the 'TSS', were not able to show significant increase in activity of Aurora $B$ in the $1 \mathrm{X}+4 \mathrm{X}(\mathrm{I})$ mixture. This suggests that a domain outside the catalytic subunit of CPC mediates the auto-activation. This is supported by the fact that Dasra/ Borealin has a dimerization domain that is regulated through Mps1 phosphorylation (Bourhis et al., 2009) and we will discuss this in the following section in detail.

Mps1, a dual specificity mitotic kinase involved in spindle checkpoint signaling and centrosome duplication (Liu and Winey, 2012), phosphorylates Borealin to stimulate Aurora B activity (Jelluma et al., 2008). Moreover, phosphorylation of Thr 230 of Borealin, one of 4 residues phosphorylated by Mps1, affects dimerization of Borealin (Bourhis et al., 2009). It is also shown that inhibiting Aurora B activity by small molecule inhibitor ZM447439 results in failure to recruit Mps1 to the kinetochores and impaired spindle checkpoint signaling (Saurin et al., 2011). Moreover, Mps1 recruits Bub1, which phosphorylates Histone H2AT120 that is one of two histone phosphorylation marks required for $\mathrm{CPC}$ recruitment to the inner centromere. Together these data shows that there is a complex crosstalk between Mps1 and Aurora B. We were interested in understanding the mechanism by which Dasra/ Borealin phosphorylation by Mps1 regulates Aurora B activity. We have shown that $\lambda$-phosphatase treated inactive CPC 
requires a small amount of active CPC to be re-activated. A similar effect was observed by adding Mps1 to $\lambda$-phosphatase treated inactive CPC (Fig. 3-2A-C). Together these data suggests that Mps1 can provide the initial trigger to activate CPC and then concentration dependent auto-activation can generate a higher activity state for Aurora B required to drive most inner centromeric events.

Since CPC localizes to chromatin for the longest duration during mitosis (Ruchaud et al., 2007) we also tested if DNA had a stimulatory effect on Aurora B kinase activity. We observed that DNA could generate the auto-activation process at 5nM CPC concentration. Although titration of CPC showed that in absence of any cofactor a higher concentration was required to generate this auto-activation, adding very low amounts ( $<50 \mathrm{fmol}$ ) plasmid DNA to 5nM CPC stimulated CPC activity by 3-fold (Fig. 3-2F,F'). This effect gradually got saturated and then adding more than $200 \mathrm{fmol}$ plasmid caused complete loss of this stimulation. We reasoned that fewer plasmids allowed a local enrichment of CPC molecules mimicking a higher concentration state, which got spread out to multiple plasmid at higher plasmid concentrations thus losing this local enrichment. This supports the concentration dependent auto-activation model. Adding DNA to CPC activation assays increased the kinetics of $1 \mathrm{X}+4 \mathrm{X}(\mathrm{I})$ mixture more than 2fold over $1 \mathrm{X}+4 \mathrm{X}(\mathrm{I})$ alone (Fig. 3-2H). Finally, we tested addition of microtubules to our assay and saw a similar effect (Fig. 3-2J). It was not surprising as CPC has multiple microtubule binding domains and supports the idea of local enrichment of multiple CPC molecules that can drive the auto-activation process. Together these data suggest that CPC auto-activates Aurora B kinase activity in a concentration dependent manner. This also suggests that there are multiple ways to trigger CPC activity. But a common thread 
here is that a condition that allows enrichment of CPC molecules drives the autoactivation reaction.

Aurora B N-terminus has an internal off-switch

We have focused on the N-terminal (1-60) domain of Xenopus Aurora B since it is largely unexplored. The crystal structure of Xenopus Aurora B lacks this region because it is unstructured (Sessa et al., 2005). We show that loss of this region causes reduction in specific activity of Aurora B kinase. Moreover we have determined that auto-phosphorylation of Thr 56 is required for reaching this higher activity state that is missing in the N-terminal truncated version. But there is another Theronine residue in that N-terminal domain that plays an equally crucial and unexpected role. We find that prolonged pre-incubation of Aurora B kinase with ATP $/ \mathrm{Mg}^{2+}$ causes reduction of kinase activity. This reduction is at least partially attributed to phosphorylation of Thr 18 of the $\mathrm{N}$-terminal tail domain. Mutating this threonine to alanine generates a more active Aurora B than wildtype (Fig. 3-3F). This suggests that Aurora B has an internal 'off switch' which is triggered during prolonged auto-activation in vitro. The next step is to determine whether this is true in vivo. The $\mathrm{N}$-terminal domain is highly divergent and this site is not conserved in mammals. But a careful analysis of the $\mathrm{N}$-terminal tail region might open up previously unknown modes of Aurora B regulation.

Microtubule interaction and its regulation by kinase activity

CPC localizes to spindle microtubules from prometaphase till cytokinesis. Immunofluorescence imaging shows majority of CPC-microtubule interaction occurs in 
anaphase (Earnshaw and Cooke, 1991; Ruchaud et al., 2007). Our data on Aurora Btubulin PLA shows that this CPC interacts with Pre-K fibers in prometaphase (Fig. 2-5). This interaction is significant since microtubules are a known co-factor of Aurora B kinase that stimulate its activity (Rosasco-Nitcher et al., 2008). Here we show how CPC as a complex interacts with microtubules and suggest a mechanistic model where this interaction can be modulated by Aurora B activity. There are two microtubule binding domains on CPC, one in the INCENP central coiled-coil domain (Mackay et al., 1998) and another in the catalytic domain (Rosasco-Nitcher et al., 2008). Electron microscopy data of CPC with taxol-stabilized microtubule suggest that CPC forms two types of structures on microtubules. It wraps around the protofilaments in a spiral or forms clusters of oligomers that protrude at fixed angles from the microtubule surface (Fig. 35E). Since it is hard to study microtubule binding through multiple binding domains we focused on the microtubule binding properties of the less well-characterized catalytic subunit. Our data suggested that auto-phosphorylation negatively regulates affinity of this domain for microtubules.

We speculate that this interaction might be electrostatic in nature and we propose that the negatively charged phosphates incorporated by auto-phosphorylation reduce the affinity between Aurora B and microtubules. Moreover, analyzing the crystal structure of Xenopus Aurora B we have identified key arginines (R) and lysines (K), that are probably mediating this interaction, that form two distinct positive patches on its surface (Fig. 3-7). This supports our hypothesis since microtubules are negatively charged polymers. If our hypothesis is correct, mutating these residues to glutamic acid (E) should reduce the affinity of $\mathrm{AI}^{790-856}$ towards microtubules. An alternate hypothesis is that a 
conformational change in fully active kinase alters the affinity of $\mathrm{AI}^{790-856}$ for microtubules. 

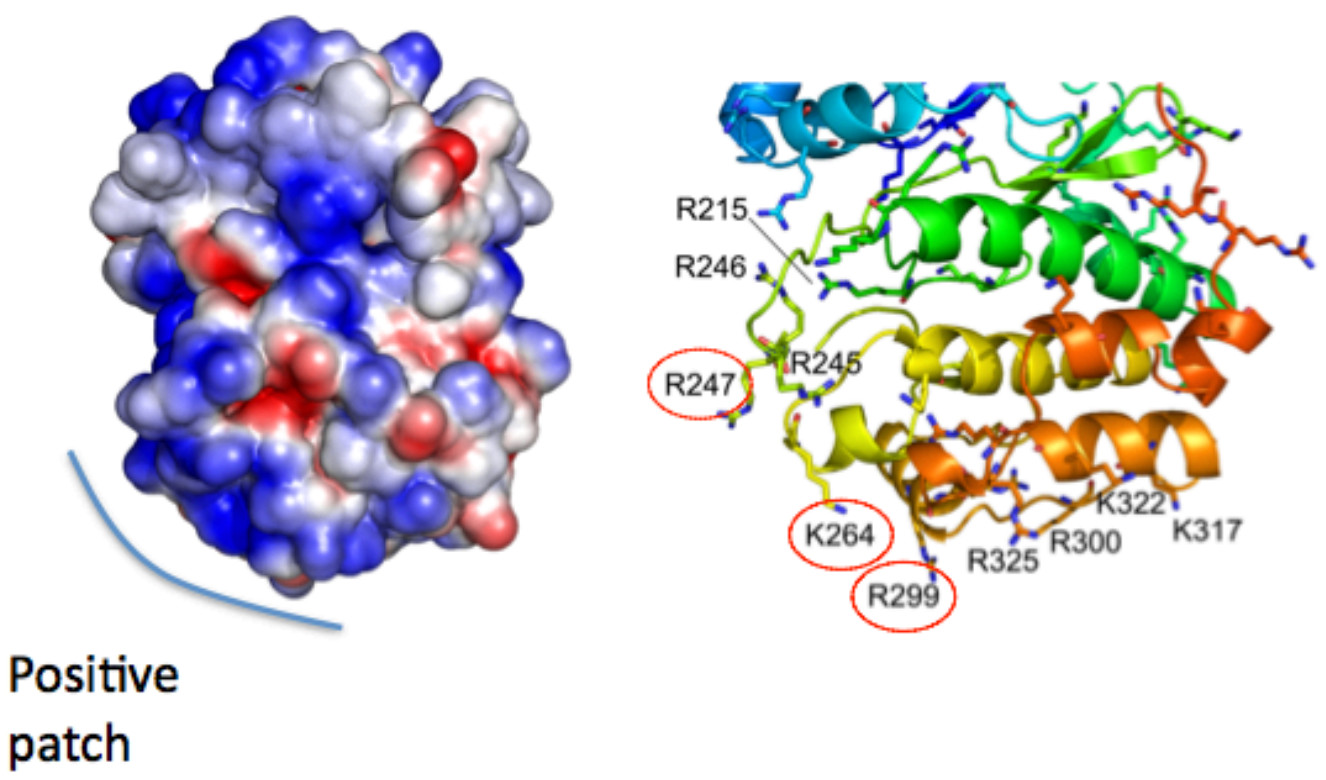

\section{patch}

Figure 3-7: Surface charge distribution of AI $^{790-847}$ shows positively charged patch.

(Left) Space filling depiction of Xenopus Aurora B in complex with INCENP C-terminal IN-Box C-Lobe showing surface charge distribution. A positively charged patch depicted as the putative microtubule binding domain. (Right) Zoom in of the C-Lobe of crystal structure of Xenopus Aurora B in complex with INCENP C-terminal IN-Box. The arginine $(\mathrm{R})$ residues highlight in red circles constitute the positive patch on the putative microtubule binding domain. 


\section{Materials and methods}

Cloning and site-directed mutagenesis

We replaced the $\Delta \mathrm{N} 60$-Aurora $\mathrm{B}$ with full length Aurora B in the pGEX6P2- $\triangle N 60$ AuroraB-INCENP ${ }^{790-847 / 856}$ constructs (Sessa et al., 2005). Full length $x$ A Aurora B was PCR amplified out of a pET28-xAurora B clone present in lab. This was done with oligos that carried restriction digestion sites matched to cut out $\Delta \mathrm{N} 60$-Aurora $\mathrm{B}$ (Cla1, BamH1) from the constructs. Both the pGEX6P2- $\triangle$ N60AuroraB-INCENP ${ }^{790-847 / 856}$ constructs and the full length Aurora B PCR amplified fragment were digested with ClaI and BamHI and agarose gel purified. Both the $\triangle$ N60-AuroraB-INCENP ${ }^{790-847 / 856}$ construct backbone had intact INCENP-IN-Box cassettes. It was ligated with the FL-Aurora $\mathrm{B}^{\text {ClaI,BamHI }}$ insert to get the desired constructs. Eventually both the bi-cistronic cassettes were PCR amplified out and inserted into pMCSG7 vector by Ewa Niedzialkowska in the lab to increase the yield. pMCSG7-AuroraB-INCENP ${ }^{790-856}$ and pMCSG7-AuroraBINCENP $^{790-847}$ constructs were modified by QuickChange mutagenesis kits (Agilent) to generate K122R-Aurora B kinase dead version of the construct. Other point mutants on Aurora B - T18A, T56A and INCENP - T848A, S849A, S850A were also generated by QuickChange mutagenesis kit (Agilent).

\section{Recombinant protein purification}

Chromosome passenger complex (CPC) - CPC was purified by co-transforming two bicistronic constructs simultaneously into BL21pLysS E.coli strain. Since pET28bINCENP-AuroraB-6His carried kanamycin resistance cassette and pET15b-SurvivinDasraA carried ampicillin resitance cassette we were able to select for clones that got 
both the constructs by plating the transformants on Kan+Amp LB-agar plates. To purify recombinant $\mathrm{CPC}, 100 \mathrm{ml}$ liquid culture was inoculated with a singe colony and grown overnight with Kan+Amp. This liquid culture was used next day to inoculate 6 liters of 2X-YT, again with Kan+Amp. Cultures were grown to $0.8 \mathrm{OD}$ and cells were collected, lysed by sonication and purified with Ni-NTA agarose (Qiagen) beads. Since only Aurora B had the 6-His tag we expected to purify other CPC protein bound to Aurora B in a complex. Elute from Ni-6His affinity purification were then passed over a Mono-Q column and fraction containing the CPC proteins was collected. This was further purified by size-exclusion chromatography by passing over a Superose 6 column in $200 \mathrm{mM} \mathrm{NaCl}$, 20mM Tris-pH 7.5, 1mM DTT, $1 \mathrm{mM}$ EDTA and 5\% glycerol. CPC was purified to homogeneity and concentrated to a final concentration of $0.3 \mathrm{mg} / \mathrm{ml}$.

FL-AuroraB:INCENP $P^{790-856}$ and FL-AuroraB:INCENP $P^{790-847}$

pGEX6P2-AuroraB-INCENP ${ }^{790-856}$ and pGEX6P2-AuroraB-INCENP ${ }^{790-847}$ were purified from BL21pLysS E.coli strain using the GST-tag on AuroraB-N-terminus (Sessa et al., 2005). Precission protease cleaved product was run over a Supedex 200 column and $\mathrm{AI}^{790-856}(0.3 \mathrm{mg} / \mathrm{ml})$ and $\mathrm{AI}^{790-847}(1 \mathrm{mg} / \mathrm{ml})$ were purified to homogeneity. pMCSG7 constructs carried a N-terminal 6-His tag on Aurora B instead of GST. All forms of AI proteins from the pMCSG7- constructs were purified from Arctic cells that were induced by IPTG at $12^{\circ} \mathrm{C}$. Following steps of purification was same as CPC purification explained above with the only exception of omitting the MonoQ-column run. Superdex 200 column was used for the size-exclusion chromatography, instead of Superose 6. 


\section{Microtubule polymerization}

Phosphocellulose (PC)- purified bovine tubulin was polymerized to microtubues using taxol as described (Desai et al., 1999). Microtubules were resuspended in dilution buffer supplemented with an equimolar concentration of taxol $(20-40 \mu \mathrm{M})$.

\section{Microtubule cosedimentation assay}

To determine the microtubule affinity of $\mathrm{CPC}$ or $\mathrm{AI}$ variants cosedimentation assays were performed using taxol stabilized microtubules in equimolar taxol-dilution buffer as previously described (Cheeseman et al., 2006). Cosedimented protein as detected by western blotting using anti-xlAuroraB (Rb615) and anti-tubulin (Dm1 $\alpha$, Sigma) antibodies. HRP signal was detected using FluoroChem FC2 imager (Alpha Innotech) and signal intensity was measured using densitometry tool in FC2 software. Percent protein bound to tubulin was measured by comparing to total amount of protein mixed with microtubules run as load. Microsoft Excel was used to plot the data.

\section{Lambda-Phosphatase treatment}

Recombinant protein was mixed with Lambda-Phosphatase (NEB) in phosphatase enzyme buffer $/ \mathrm{Mn}^{2+}$. Reaction was carried out for 60 minutes at RT and then freshly prepared $10 \mu \mathrm{M}$ Sodium-ortho-vanadate was added to stop reaction. To confirm Na-oVanadate was completely active a parallel reaction was performed where it was added along with Lambda-phosphatase before the 60 minutes incubation. 


\section{Kinase activity assay}

Recombinant $\mathrm{AI}^{(790-856)}$ or $\mathrm{CPC}$ was diluted to the required concentrations and incubated with either $3 \mu \mathrm{M}$ Taxol-stabilized microtubules (prepared as explained) or 1X-BRB80 (80 $\mathrm{mM}$ Pipes, $1 \mathrm{mM} \mathrm{MgCl}_{2}, 1 \mathrm{mM}$ EGTA $\mathrm{pH}-6.8$ with $\mathrm{KOH}$ ), for 15 minutes as required. Assays were carried out in kinase buffer (KB) $(20 \mathrm{mM}$ Tris- $\mathrm{HCl} \mathrm{pH}-7.5,1 \mathrm{mM} \mathrm{MgCl}$, $25 \mathrm{mM} \mathrm{KCl}, 1 \mathrm{mM}$ DTT and $100 \mu \mathrm{M} \mathrm{ATP} / 1 \mu \mathrm{M} \gamma^{32} \mathrm{P}-\mathrm{ATP}$ mix) with myelin basic protein (MBP, Invitrogen) or GST-HistoneH3 tail as substrate. Activity was initiated by incubation with microtubules/1X BRB80/KB and stopped after 2 minutes by adding SDS-PAGE sample buffer or in case of end point assay after the mentioned time (Check figure legends). Samples were separated on 15\%-SDS-PAGE gel, stained with coomassie blue, dried on Whattman paper together with aliquots of $\gamma^{32} \mathrm{P}-\mathrm{ATP}$ and exposed to Phosphor Screen (Molecular Dynamics) overnight. Phosphor Screens were scanned on a Storm-860 Phosphor-scanner (Molecular Dynamics) and resulting images were processed and quantified using ImageQuant (Molecular Dynamics) to calculate amount of $\mathrm{PO}_{4}{ }^{3-}$ incorporated on MBP. Error bars represent standard deviations.

\section{Electron Microscopy}

Electron micrograph of recombinant CPC were obtained after negative staining with uranyl acetate. The work was done in collaboration with Dr. Jay Brown (UVa. Microbiology Dept.). Formvar grids (a gift from Dr. Anne Beyer, UVa. Microbiology Dept.) were layered over with carbon and protein was applied. Buffer was applied to grids as negative control. These were then subjected to $2 \%$ Uranyl acetate treatment for 
10 minutes. Excess Uranyl acetate was removed by soaking the edges of grid on kimwipes. Electron micrographs were taken after drying them for 5 minutes. 


\section{Acknowledgement}

I would like to thank Limin Liu for critically reading this chapter. Dr. Jay Brown and Bill Newcomb performed the electron microscopy. Dr. Anne Beyer provided the grids. Ewa Niedzialkowska helped with the crystal structure analysis of $\mathrm{AI}^{790-847}$ to identify key arginines that might be involved in microtubule interaction. 
CHAPTER IV:

GENERAL DISCUSSION AND FUTURE DIRECTIONS 
Our understanding of the Chromosome passenger complex has come a long way in the last few years. The title of a recent review by Bill Earnshaw and colleagues reads "The chromosomal passenger complex: from easy rider to godfather of mitosis." (Carmena et al., 2012). One might find the names bit unorthodox but by no means are they unreasonable. It was called a 'passenger' complex based on the hypothesis that its dynamic localization pattern allows it to control various events including chromosome segregation and cytokinesis by phosphorylating key substrates in a spatially restricted manner (Deluca et al., 2006; Cheeseman and Desai, 2008; Zimniak et al., 2009; Lan et al., 2004; Knowlton et al., 2006; Miller et al., 2008; Guse et al., 2005; Bishop and Schumacher, 2002; Minoshima et al., 2003; Saurin et al., 2011; Wang et al., 2011). The term 'godfather' might be a bit cinematic but is not an exaggeration by any means. Human homologues of Aurora kinase are oncogenic and are found overexpressed in multiple primary cancers (Bischoff et al., 1998; Giet and Prigent, 1999). A recent work showed that Aurora B phosphorylates p53 to accelerate its degradation by polyubiquitination-proteosome pathway (Gully et al., 2012). Thus it has become an attractive target for cancer therapies and research.

\section{Significance}

The work presented in this thesis was designed to characterize CPC regulation both in vivo and in vitro. We have collaborated to identify that a phospho-histone mark on histone $\mathrm{H} 3 \mathrm{Thr} 3$ is required to recruit $\mathrm{CPC}$ to the centromere. Moreover, we have identified a novel pathway involving EB1/ microtubules that is required for CPC recruitment in prometaphase, which is upstream of the histone phosphorylation pathways. 
This suggests a mechanism by which the mitotic spindle can regulate Aurora B levels at the centromeres. We have also demonstrated that CPC can auto-activate Aurora B kinase activity in a concentration dependent manner and this requires a domain outside the catalytic subunit of $\mathrm{CPC}$. We show that DNA can stimulate the auto-activation reaction and it is through local enrichment of CPC molecules. We have identified new autophosphorylation sites on Xenopus Aurora B N-terminus that regulates its kinase activity. Finally we have analyzed the microtubule-binding domain within the catalytic subunit and demonstrated the phospho-regulation of this microtubule binding. These are significant contributions to the field that opens up new paradigms for understanding CPC regulation. In the following section I will highlight some exciting implications of my work and discuss some unanswered questions and future directions.

\section{Complexity of Aurora B regulation}

A key feature of Aurora B kinase is that it does not merely exist at ON or OFF state, but its activity is modulated in response to its immediate environment. In addition to T-loop phosphorylation, there are numerous activating phosphorylations on other CPC proteins that fine tune Aurora B activity (Bishop and Schumacher, 2002; Sessa et al., 2005; Jelluma et al., 2008; Bolton et al., 2002; Chu et al., 2011). It is suggested that various phosphorylation states of the 'TSS' motif generates different levels of Aurora B activity (Xu et al., 2010). Moreover, it is proposed that Aurora B recognizes the errors in kinetochore-microtubule attachment by sensing the proximity of kinetochore substrates in response to tension exerted by the kinetochore microtubules (Liu et al., 2009). Our data demonstrates that Aurora B levels are regulated by EB1/ microtubule and the active 
kinase is not merely anchored at the centromeres. Our model suggests a mechanism where the spindle communicates directly with the centromeres to modulate Aurora B levels and activity at the kinetochores in response to the status of microtubule attachment. Microtubule can stimulate Aurora B kinase activity in vitro and treating anaphase cells with microtubule depolymerizing drug nocodazole for 8 minutes results in loss of Aurora B activating phosphorylations on INCENP (Fuller et al., 2008). It is reasonable to think that the presence of preformed $\mathrm{K}$-fibers at the kinetochores will stimulate Aurora B activity, which can drive enrichment of more CPC by inducing phosphorylation of histone H3T3 and H2AT120 by the feedback loops (Saurin et al., 2011; Wang et al., 2011). Together, these data suggest that Aurora B integrates information from kinetochores, cohesion related proteins and spindle microtubules. In response to this it generates output signals to activate spindle checkpoint and initiate kinetochoremicrotubule attachment error correction pathways by activating MCAK (Figure 4-1). This also defines the complexity of Aurora B regulation and hence it is important to carry out in vivo and in vitro studies to demonstrate the role of each regulator.

We also tested if these feedback loops, that drive enrichment of CPC at centromeres, can function independent of each other (Figure 2-9). Our data suggests that inhibiting Haspin has little effect on Mps1-Bub1-Sgo1 axis as long as Aurora B activity is not diminished. We would also like to know if the opposite is also true. Similar to the previous experiment, we can treat CenpB-INCENP expressing cells with Mps1 inhibitor reversine (Santaguida et al., 2011; van der Waal et al., 2012) and assay phospho-H3T3 levels by immunofluorescence. Initial findings suggest that the only common component in the functioning of these two loops is Aurora B. 


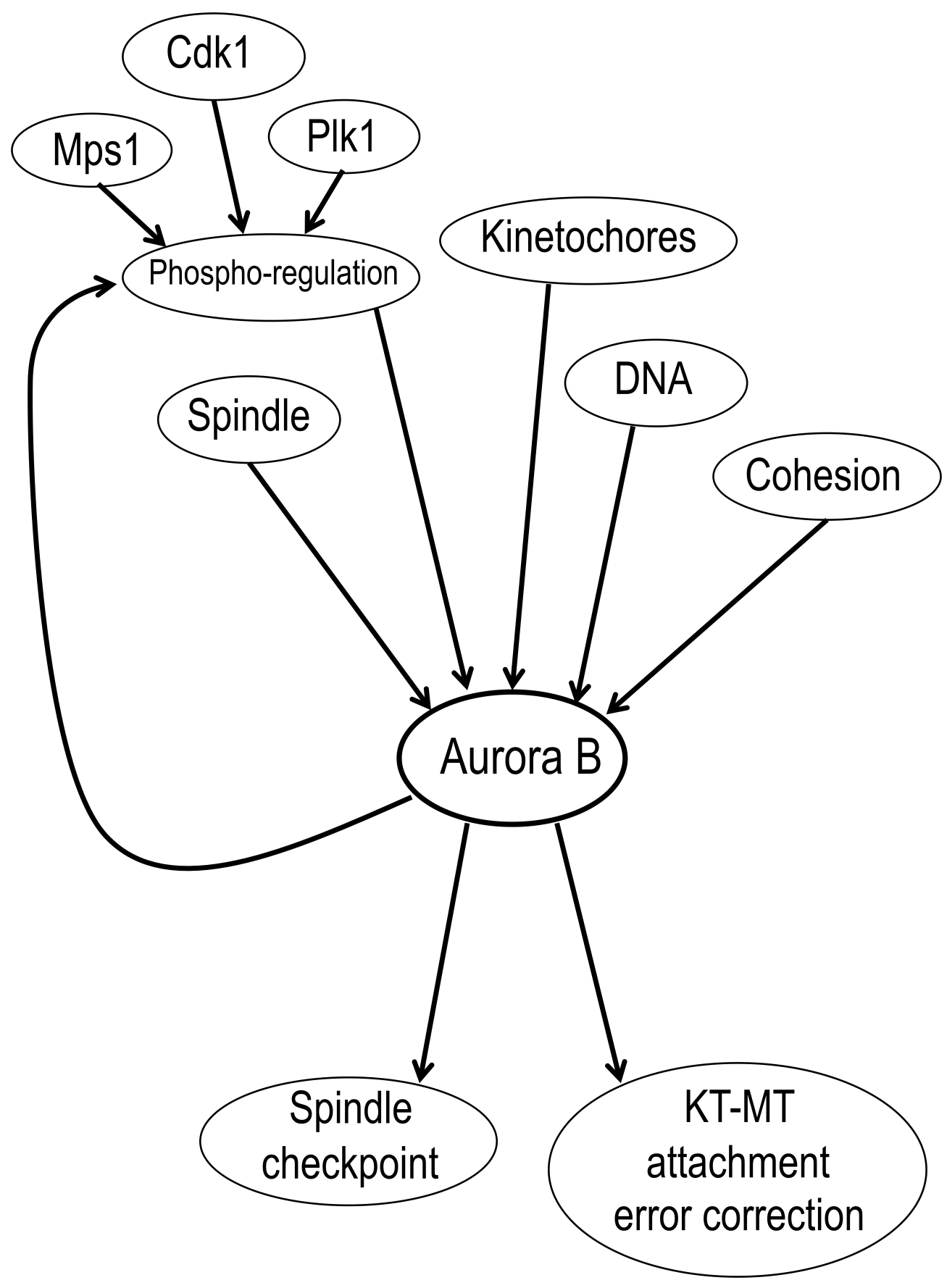

Figure 4-1: Complexity of Aurora B regulation

Schematic representation of various input signals that determine Aurora B mediated signaling of spindle checkpoint and error correction pathways. 


\section{CPC in late mitosis}

There is a gradient of soluble Aurora B activity centered around the spindle midzone in anaphase (Fuller et al., 2008). CPC relocates from centromeres to midzone microtubule bundles during anaphase onset and then localizes to the equatorial cortex shortly thereafter (Earnshaw and Cooke, 1991). It is essential to remove the CPC from chromatin for mitotic exit and reformation of the nucleus (Vagnarelli et al., 2011). Removal of CPC from chromatin is coupled with cell cycle progression, decrease in Cdk1 activity and interactions with microtubule associated motor proteins MKLP2 / Bim1 (Hümmer and Mayer, 2009; Nakajima et al., 2011). Our data suggest that Aurora B activity might play an essential role in generating the soluble pool of active Aurora B. Microtubule binding affinity of the catalytic subunit of CPC is inversely correlated with its activity state (Figure 3-4). It suggests a possible mechanism where Aurora B in a low activity state would bind microtubules to get activated, which would then allow it to release from microtubules by auto-phosphorylation, to diffuse into the active soluble pool. Moreover, EB1 depletion in HeLa cells disrupts the Aurora B activity gradient as visualized by measuring chromatin substrate phosphorylation on lagging chromosomes (Figure 2-10). This not only shows EB1's requirement for the anaphase activity gradient but also suggests that Aurora B-microtubule association might be critical in establishing it. This is supported by our PLA experiments to identify Aurora B-microtubule interactions in anaphase. We observe numerous PLA spots on astral and cortical microtubules that disappear on depolymerizing the microtubules by cold treatment. This suggests that Aurora B interacts with microtubules all over the spindle and is not restricted to the midzone, although midzone localizations are also picked up by PLA 
(data not shown). The EB1 (K89E) point mutant is deficient in microtubule binding. It will be interesting to see if this mutant can rescue the EB1 depletion phenotype of disrupting the Aurora B anaphase gradient. Similarly we are making point mutants in Xenopus Aurora B that disrupts microtubule binding. The residues are conserved in human Aurora B so we can do knockdown replacement experiments, which will test the role of this interaction in anaphase. More careful work needs to be done to determine the role of EB1/microtubules in generating the Aurora B activity gradient in anaphase.

Does EB1-Aurora B regulate chromosome oscillations in metaphase?

EB1 is the only protein that localizes specifically to the sister kinetochores that have growing microtubule ends (anti-poleward) (Tirnauer et al., 2002). This finding combined with our data suggest that Aurora B activity would be higher on the antipoleward sister kinetochores. Consistent with this idea most PLA signals are typically found near one of the two sister kinetochores (Figure 2-4E). Differential phosphorylation on the poleward and anti-poleward sides could provide mechanisms to coordinate the two sisters to allow proper chromosome movements (Liu et al., 2009; Tirnauer et al., 2002; Dumont et al., 2012). The Skal complex, an important component of the kinetochore, has the ability to track depolymerizing microtubules and binds curved protofilaments (Schmidt et al., 2012). Recent work from a colleague in the lab shows PP1 interacts with Ska1 and is recruited to the kinetochores (Pawel Janczyk, unpublished). This suggests a possible mechanism of how chromosomes oscillate by generating completely different biochemical states at the two kinetochores, separated by a few microns. The kinetochore attached to the depolymerizing microtubules (poleward) recruits PP1 through Ska1 
binding and maintain kinetochore proteins at an un-phosphorylated state allowing them to track the shrinking microtubules. Simultaneously, EB1 being a plus end tracking protein, will enrich on the sister kinetochore bound to the polymerizing microtubule (antipoleward). This will allow concentration of Aurora B at this kinetochore, which will phosphorylate kinetochore proteins like Ndc80 and KNL1 thus allowing them to slide on growing microtubules without forming stable attachments like a 'clutch' (Figure 4-2). Although this model explains how the asymmetry of two kinetochores is generated, which is required for each phase of oscillation, it does not explain how the switch in direction occurs.

I have made Aurora B point mutants targeting two putative EB1 binding motifs. EB1 recognizes SXIP motifs on its cargo for interaction and human Aurora B has three such motifs. We will test if mutating STL ${ }^{(24)} \mathrm{P}^{(25)}$ to STNN and $\mathrm{SGT}^{(64)} \mathrm{P}^{(65)}$ to SGNN perturb EB1 binding by pull-down assays or isothermal calorimetry (ITC). We will also perform co-immunoprecipitation assays and live cell time-lapse imaging with cell lines transfected with these Aurora B mutants (LAP-tagged), stably expressing shRNA targeted against the endogenous gene. This would test our hypothesis that EB1 dependent Aurora B enrichment on anti-poleward kinetochores establishes the asymmetry that is required for chromosome movements. 


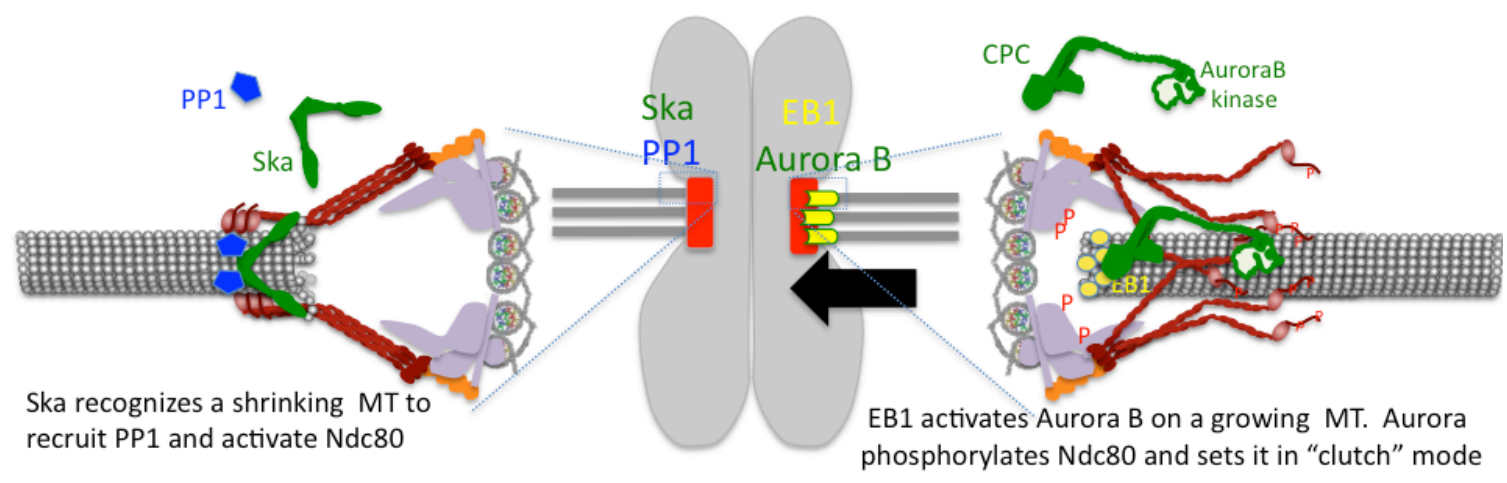

Figure 4-2: A model for how differential phosphorylation of sister kinetochores emanates from the polymerization state of the end-on bound microtubule.

On the anti-poleward kinetochores the growing microtubules recruit EB1 and stimulate Aurora B to phosphorylate kinetochores. On the poleward kinetochore the curling ends of depolymerizing microtubules recruit Ska/PPI. These differences in phosphorylation drive the asymmetry of sister kinetochore activity (shown as a change in Ndc80 attachment) and allow sister kinetochores to rapidly change states during oscillations. 


\section{CPC localization to centromeres and the role of phosphatases}

The phospho-histone marks that are essential to recruit the CPC to the centromeres are not constitutive. They appear in prophase and are gone after anaphase onset. CPC relocalizes to the midzone microtubules and histone H3Thr3 is removed by PP1 (Qian et al., 2013; Vagnarelli et al., 2011). Aurora B regulates its localization very tightly. It induces Mps1 and Haspin kinase associated loops (Saurin et al., 2011; Wang et al., 2011) and simultaneously phosphorylates Repo-man, a nuclear protein that binds and regulates PP1 $\gamma$, on Ser 893 to restrict its centromeric localization thus keeping PP1 away (Qian et al., 2013). This reciprocal feedback regulation of Haspin and Repo-man generates a robust bi-stable response to enrich CPC to the centromeres in prometaphase. Aurora B activity is high at the centromeres, which would favor destabilization of kinetochore microtubule attachment. Then how do unattached chromosomes contact microtubules? BubR1 associated B56-PP2A phosphatase is recruited to the centromeres and has been implicated in antagonizing Aurora B, which regulates kinetochore substrate phosphorylation and generate stable kinetochore-microtubule attachments (Foley et al., 2011). But the interplay between Aurora B recruiting phospho-histone marks and this antagonistic phosphatase in prometaphase is not known and remains an intriguing question. EB1 has been shown to interact with Aurora B and this interaction inhibits dephosphorylation of Aurora B T-loop by PP2A (Sun et al., 2008). Our findings on EB1's role in recruiting $\mathrm{CPC}$ in prometaphase along with its role in antagonizing PP2A suggest a possible mechanism that balance B56-PP2A activity recruited to centromeres with CPC functions. 


\section{Reference:}

Adams, R.R., H. Maiato, W.C. Earnshaw, and M. Carmena. 2001a. Essential roles of Drosophila inner centromere protein (INCENP) and aurora B in histone $\mathrm{H} 3$ phosphorylation, metaphase chromosome alignment, kinetochore disjunction, and chromosome segregation. J. Cell Biol. 153:865-880.

Adams, R.R., M. Carmena, and W.C. Earnshaw. 2001b. Chromosomal passengers and the (aurora) ABCs of mitosis. Trends Cell Biol. 11:49-54.

Adams, R.R., S.P. Wheatley, A.M. Gouldsworthy, S.E. Kandels-Lewis, M. Carmena, C. Smythe, D.L. Gerloff, and W.C. Earnshaw. 2000. INCENP binds the Aurora-related kinase AIRK2 and is required to target it to chromosomes, the central spindle and cleavage furrow. Curr. Biol. 10:1075-1078.

Ambrosini, G., C. Adida, and D.C. Altieri. 1997. A novel anti-apoptosis gene, survivin, expressed in cancer and lymphoma. Nat. Med. 3:917-921.

Andrews, P.D., Y. Ovechkina, N. Morrice, M. Wagenbach, K. Duncan, L. Wordeman, and J.R. Swedlow. 2004. Aurora B regulates MCAK at the mitotic centromere. Dev Cell. 6:253-268.

Bayliss, R., T. Sardon, I. Vernos, and E. Conti. 2003. Structural basis of Aurora-A activation by TPX2 at the mitotic spindle. Mol Cell. 12:851-862.

Biggins, S., and A.W. Murray. 2001. The budding yeast protein kinase Ipl1/Aurora allows the absence of tension to activate the spindle checkpoint. Genes \& Development. 15:3118-3129. doi:10.1101/gad.934801.

Bischoff, J.R., L. Anderson, Y. Zhu, K. Mossie, L. Ng, B. Souza, B. Schryver, P. Flanagan, F. Clairvoyant, C. Ginther, C.S. Chan, M. Novotny, D.J. Slamon, and G.D. Plowman. 1998. A homologue of Drosophila aurora kinase is oncogenic and amplified in human colorectal cancers. EMBO J. 17:3052-3065. doi:10.1093/emboj/17.11.3052.

Bishop, J.D., and J.M. Schumacher. 2002. Phosphorylation of the carboxyl terminus of inner centromere protein (INCENP) by the Aurora B Kinase stimulates Aurora B kinase activity. J. Biol. Chem. 277:27577-27580. doi:10.1074/jbc.C200307200.

Bolton, M.A., W. Lan, S.E. Powers, M.L. McCleland, J. Kuang, and P.T. stukenberg. 2002. Aurora B kinase exists in a complex with survivin and INCENP and its kinase activity is stimulated by survivin binding and phosphorylation. Mol. Biol. Cell. 13:30643077. doi:10.1091/mbc.E02-02-0092.

Bourhis, E., A. Lingel, Q. Phung, W.J. Fairbrother, and A.G. Cochran. 2009. Phosphorylation of a Borealin Dimerization Domain Is Required for Proper Chromosome 
Segregation. Biochemistry. 48:6783-6793. doi:10.1021/bi900530v.

Boveri, T. 1914. Zur Frage der Entstehung maligner Tumoren. Science. 40:857-859. doi:10.1126/science.40.1041.857.

Brito, D.A., Z. Yang, and C.L. Rieder. 2008. Microtubules do not promote mitotic slippage when the spindle assembly checkpoint cannot be satisfied. J. Cell Biol. 182:623629. doi: $10.1083 /$ jcb.200805072.

Carmena, M., and W.C. Earnshaw. 2003. The cellular geography of aurora kinases. Nat Rev Mol Cell Biol. 4:842-854. doi:10.1038/nrm1245.

Carmena, M., M. Wheelock, H. Funabiki, and W.C. Earnshaw. 2012. The chromosomal passenger complex (CPC): from easy rider to the godfather of mitosis. Nat Rev Mol Cell Biol. 13:789-803. doi:10.1038/nrm3474.

Chan, C.S., and D. Botstein. 1993. Isolation and characterization of chromosome-gain and increase-in-ploidy mutants in yeast. Genetics. 135:677-691.

Chantalat, L., D.A. Skoufias, J.P. Kleman, B. Jung, O. Dideberg, and R.L. Margolis. 2000. Crystal structure of human survivin reveals a bow tie-shaped dimer with two unusual alpha-helical extensions. Mol Cell. 6:183-189.

Cheeseman, I.M., and A. Desai. 2008. Molecular architecture of the kinetochoremicrotubule interface. Nat Rev Mol Cell Biol. 9:33-46. doi:10.1038/nrm2310.

Cheeseman, I.M., J.S. Chappie, E.M. Wilson-Kubalek, and A. Desai. 2006. The conserved KMN network constitutes the core microtubule-binding site of the kinetochore. Cell. 127:983-997. doi:10.1016/j.cell.2006.09.039.

Cheeseman, I.M., S. Anderson, M. Jwa, E.M. Green, J.S. Kang, J.R. Yates, C.S.M. Chan, D.G. Drubin, and G. Barnes. 2002. Phospho-regulation of kinetochore-microtubule attachments by the Aurora kinase Ipl1p. Cell. 111:163-172.

Chu, Y., P.Y. Yao, W. Wang, D. Wang, Z. Wang, L. Zhang, Y. Huang, Y. Ke, X. Ding, and X. Yao. 2011. Aurora B kinase activation requires survivin priming phosphorylation by PLK1. J Mol Cell Biol. 3:260-267. doi:10.1093/jmcb/mjq037.

Cimini, D., B. Moree, J.C. Canman, and E.D. Salmon. 2003. Merotelic kinetochore orientation occurs frequently during early mitosis in mammalian tissue cells and error correction is achieved by two different mechanisms. J. Cell. Sci. 116:4213-4225. doi: $10.1242 /$ jcs. 00716 .

Cimini, D., X. Wan, C.B. Hirel, and E.D. Salmon. 2006. Aurora kinase promotes turnover of kinetochore microtubules to reduce chromosome segregation errors. Curr. Biol. 16:1711-1718. doi:10.1016/j.cub.2006.07.022.

Cooke, C.A., M.M. Heck, and W.C. Earnshaw. 1987. The inner centromere protein 
(INCENP) antigens: movement from inner centromere to midbody during mitosis. $J$. Cell Biol. 105:2053-2067.

Crosio, C., G.M. Fimia, R. Loury, M. Kimura, Y. Okano, H. Zhou, S. Sen, C.D. Allis, and P. Sassone-Corsi. 2002. Mitotic phosphorylation of histone H3: spatio-temporal regulation by mammalian Aurora kinases. Mol. Cell. Biol. 22:874-885.

De Antoni, A., S. Maffini, S. Knapp, A. Musacchio, and S. Santaguida. 2012. A smallmolecule inhibitor of Haspin alters the kinetochore functions of Aurora B. J. Cell Biol. 199:269-284. doi:10.1083/jcb.201205119.

De Souza, C.P.C., and S.A. Osmani. 2007. Mitosis, not just open or closed. Eukaryotic Cell. 6:1521-1527. doi:10.1128/EC.00178-07.

Deluca, J.G., W.E. Gall, C. Ciferri, D. Cimini, A. Musacchio, and E.D. Salmon. 2006. Kinetochore microtubule dynamics and attachment stability are regulated by Hec1. Cell. 127:969-982. doi:10.1016/j.cell.2006.09.047.

Desai, A., A. Murray, T.J. Mitchison, and C.E. Walczak. 1999. The use of Xenopus egg extracts to study mitotic spindle assembly and function in vitro. Methods Cell Biol. 61:385-412.

Ditchfield, C., V.L. Johnson, A. Tighe, R. Ellston, C. Haworth, T. Johnson, A. Mortlock, N. Keen, and S.S. Taylor. 2003. Aurora B couples chromosome alignment with anaphase by targeting BubR1, Mad2, and Cenp-E to kinetochores. J. Cell Biol. 161:267-280. doi:10.1083/jcb.200208091.

Draviam, V.M., I. Shapiro, B. Aldridge, and P.K. Sorger. 2006. Misorientation and reduced stretching of aligned sister kinetochores promote chromosome missegregation in EB1- or APC-depleted cells. EMBO J. 25:2814-2827. doi:10.1038/sj.emboj.7601168.

Du, J., A.E. Kelly, H. Funabiki, and D.J. Patel. 2012. Structural basis for recognition of H3T3ph and Smac/DIABLO N-terminal peptides by human Survivin. Structure. 20:185195. doi:10.1016/j.str.2011.12.001.

Dumont, S., E.D. Salmon, and T.J. Mitchison. 2012. Deformations Within Moving Kinetochores Reveal Different Sites of Active and Passive Force Generation. Science. 337:355-358.

Earnshaw, W.C., and C.A. Cooke. 1991. Analysis of the distribution of the INCENPs throughout mitosis reveals the existence of a pathway of structural changes in the chromosomes during metaphase and early events in cleavage furrow formation. J. Cell. Sci. 98 ( Pt 4):443-461.

Earnshaw, W.C., and R.L. Bernat. 1991. Chromosomal passengers: toward an integrated view of mitosis. Chromosoma. 100:139-146.

Emanuele, M.J., and P.T. stukenberg. 2007. Xenopus Cep57 is a novel kinetochore 
component involved in microtubule attachment. Cell. 130:893-905.

doi:10.1016/j.cell.2007.07.023.

Ems-McClung, S.C., K.M. Hertzer, X. Zhang, M.W. Miller, and C.E. Walczak. 2007. The interplay of the N- and C-terminal domains of MCAK control microtubule depolymerization activity and spindle assembly. Mol. Biol. Cell. 18:282-294. doi:10.1091/mbc.E06-08-0724.

Eyers, P.A., E. Erikson, L.G. Chen, and J.L. Maller. 2003. A novel mechanism for activation of the protein kinase Aurora A. Curr. Biol. 13:691-697.

Fischle, W., B.S. Tseng, H.L. Dormann, B.M. Ueberheide, B.A. Garcia, J. Shabanowitz, D.F. Hunt, H. Funabiki, and C.D. Allis. 2005. Regulation of HP1-chromatin binding by histone H3 methylation and phosphorylation. Nature. 438:1116-1122.

doi:10.1038/nature04219.

Foley, E.A., M. Maldonado, and T.M. Kapoor. 2011. Formation of stable attachments between kinetochores and microtubules depends on the B56-PP2A phosphatase. Nat. Cell Biol. 13:1265-1271. doi:10.1038/ncb2327.

Fuller, B.G., M.A. Lampson, E.A. Foley, S. Rosasco-Nitcher, K.V. Le, P. Tobelmann, D.L. Brautigan, P.T. stukenberg, and T.M. Kapoor. 2008. Midzone activation of aurora B in anaphase produces an intracellular phosphorylation gradient. Nature. 453:1132-1136. doi:10.1038/nature06923.

Ganem, N.J., Z. Storchova, and D. Pellman. 2007. Tetraploidy, aneuploidy and cancer. Curr. Opin. Genet. Dev. 17:157-162. doi:10.1016/j.gde.2007.02.011.

Gassmann, R., A. Carvalho, A.J. Henzing, S. Ruchaud, D.F. Hudson, R. Honda, E.A. Nigg, D.L. Gerloff, and W.C. Earnshaw. 2004. Borealin: a novel chromosomal passenger required for stability of the bipolar mitotic spindle. J. Cell Biol. 166:179-191.

doi:10.1083/jcb.200404001.

Giet, R., and C. Prigent. 1999. Aurora/Ipl1p-related kinases, a new oncogenic family of mitotic serine-threonine kinases. J. Cell. Sci. 112 ( Pt 21):3591-3601.

Glover, D.M., M.H. Leibowitz, D.A. McLean, and H. Parry. 1995. Mutations in aurora prevent centrosome separation leading to the formation of monopolar spindles. Cell. 81:95-105.

Green, R., R. Wollman, and K. Kaplan. 2005. APC and EB1 function together in mitosis to regulate spindle dynamics and chromosome alignment. Mol. Biol. Cell. 16:4609-4622. doi:10.1091/mbc.E05-03-0259.

Gruber, S., C.H. Haering, and K. Nasmyth. 2003. Chromosomal cohesin forms a ring. Cell. 112:765-777.

Gully, C.P., G. Velazquez-Torres, J.-H. Shin, E. Fuentes-Mattei, E. Wang, C. Carlock, J. 
Chen, D. Rothenberg, H.P. Adams, H.H. Choi, S. Guma, L. Phan, P.-C. Chou, C.-H. Su, F. Zhang, J.-S. Chen, T.-Y. Yang, S.-C.J. Yeung, and M.-H. Lee. 2012. Aurora B kinase phosphorylates and instigates degradation of p53. Proc Natl Acad Sci U S A. 109:E151322. doi:10.1073/pnas.1110287109.

Guse, A., M. Mishima, and M. Glotzer. 2005. Phosphorylation of ZEN-4/MKLP1 by aurora B regulates completion of cytokinesis. Curr. Biol. 15:778-786. doi:10.1016/j.cub.2005.03.041.

Hartwell, L.H., and T.A. Weinert. 1989. Checkpoints: controls that ensure the order of cell cycle events. Science. 246:629-634.

Hauf, S., R.W. Cole, S. LaTerra, C. Zimmer, G. Schnapp, R. Walter, A. Heckel, J. van Meel, C.L. Rieder, and J.-M. Peters. 2003. The small molecule Hesperadin reveals a role for Aurora B in correcting kinetochore-microtubule attachment and in maintaining the spindle assembly checkpoint. J. Cell Biol. 161:281-294. doi:10.1083/jcb.200208092.

Hayashi, I., and M. Ikura. 2003. Crystal structure of the amino-terminal microtubulebinding domain of end-binding protein 1 (EB1). J. Biol. Chem. 278:36430-36434. doi:10.1074/jbc.M305773200.

Hendzel, M.J., Y. Wei, M.A. Mancini, A. Van Hooser, T. Ranalli, B.R. Brinkley, D.P. Bazett-Jones, and C.D. Allis. 1997. Mitosis-specific phosphorylation of histone H3 initiates primarily within pericentromeric heterochromatin during G2 and spreads in an ordered fashion coincident with mitotic chromosome condensation. Chromosoma. 106:348-360.

Hirota, T., J.J. Lipp, B.-H. Toh, and J.-M. Peters. 2005. Histone H3 serine 10 phosphorylation by Aurora B causes HP1 dissociation from heterochromatin. Nature. 438:1176-1180. doi:10.1038/nature04254.

Honda, R., R. Körner, and E.A. Nigg. 2003. Exploring the functional interactions between Aurora B, INCENP, and survivin in mitosis. Mol. Biol. Cell. 14:3325-3341. doi:10.1091/mbc.E02-11-0769.

Hoyt, M.A., L. Totis, and B.T. Roberts. 1991. S. cerevisiae genes required for cell cycle arrest in response to loss of microtubule function. Cell. 66:507-517.

Hsu, J.Y., Z.W. Sun, X. Li, M. Reuben, K. Tatchell, D.K. Bishop, J.M. Grushcow, C.J. Brame, J.A. Caldwell, D.F. Hunt, R. Lin, M.M. Smith, and C.D. Allis. 2000. Mitotic phosphorylation of histone $\mathrm{H} 3$ is governed by Ipl1/aurora kinase and Glc7/PP1 phosphatase in budding yeast and nematodes. Cell. 102:279-291.

Hunter, A.W., M. Caplow, D.L. Coy, W.O. Hancock, S. Diez, L. Wordeman, and J. Howard. 2003. The kinesin-related protein MCAK is a microtubule depolymerase that forms an ATP-hydrolyzing complex at microtubule ends. Mol Cell. 11:445-457.

Jelluma, N., A.B. Brenkman, N.J.F. van den Broek, C.W.A. Cruijsen, M.H.J. van Osch, 
S.M.A. Lens, R.H. Medema, and G.J.P.L. Kops. 2008. Mps1 phosphorylates Borealin to control Aurora B activity and chromosome alignment. Cell. 132:233-246.

doi:10.1016/j.cell.2007.11.046.

Jeyaprakash, A.A., C. Basquin, U. Jayachandran, and E. Conti. 2011. Structural basis for the recognition of phosphorylated histone h3 by the survivin subunit of the chromosomal passenger complex. Structure. 19:1625-1634. doi:10.1016/j.str.2011.09.002.

Jeyaprakash, A.A., U.R. Klein, D. Lindner, J. Ebert, E.A. Nigg, and E. Conti. 2007. Structure of a Survivin-Borealin-INCENP core complex reveals how chromosomal passengers travel together. Cell. 131:271-285. doi:10.1016/j.cell.2007.07.045.

Kaitna, S., M. Mendoza, V. Jantsch-Plunger, and M. Glotzer. 2000. Incenp and an aurora-like kinase form a complex essential for chromosome segregation and efficient completion of cytokinesis. Curr. Biol. 10:1172-1181.

Kallio, M.J., M.L. McCleland, P.T. stukenberg, and G.J. Gorbsky. 2002. Inhibition of aurora B kinase blocks chromosome segregation, overrides the spindle checkpoint, and perturbs microtubule dynamics in mitosis. Curr. Biol. 12:900-905.

Kapoor, T.M., T.U. Mayer, M.L. Coughlin, and T.J. Mitchison. 2000. Probing spindle assembly mechanisms with monastrol, a small molecule inhibitor of the mitotic kinesin, Eg5. J. Cell Biol. 150:975-988.

Kelly, A.E., C. Ghenoiu, J.Z. Xue, C. Zierhut, H. Kimura, and H. Funabiki. 2010. Survivin reads phosphorylated histone $\mathrm{H} 3$ threonine 3 to activate the mitotic kinase Aurora B. Science. 330:235-239. doi:10.1126/science.1189505.

Kelly, A.E., S.C. Sampath, T.A. Maniar, E.M. Woo, B.T. Chait, and H. Funabiki. 2007. Chromosomal enrichment and activation of the aurora B pathway are coupled to spatially regulate spindle assembly. Dev Cell. 12:31-43. doi:10.1016/j.devcel.2006.11.001.

Khodjakov, A., L. Copenagle, M.B. Gordon, D.A. Compton, and T.M. Kapoor. 2003. Minus-end capture of preformed kinetochore fibers contributes to spindle morphogenesis. J. Cell Biol. 160:671-683. doi:10.1083/jcb.200208143.

Klein, U.R., E.A. Nigg, and U. Gruneberg. 2006. Centromere targeting of the chromosomal passenger complex requires a ternary subcomplex of Borealin, Survivin, and the N-terminal domain of INCENP. Mol. Biol. Cell. 17:2547-2558.

doi:10.1091/mbc.E05-12-1133.

Kline-Smith, S.L., A. Khodjakov, P. Hergert, and C.E. Walczak. 2004. Depletion of centromeric MCAK leads to chromosome congression and segregation defects due to improper kinetochore attachments. Mol. Biol. Cell. 15:1146-1159. doi:10.1091/mbc.E0308-0581.

Knauer, S.K., C. Bier, N. Habtemichael, and R.H. Stauber. 2006. The Survivin-Crm1 interaction is essential for chromosomal passenger complex localization and function. 
EMBO Rep. 7:1259-1265. doi:10.1038/sj.embor.7400824.

Knowlton, A.L., W. Lan, and P.T. stukenberg. 2006. Aurora B is enriched at merotelic attachment sites, where it regulates MCAK. Curr. Biol. 16:1705-1710. doi:10.1016/j.cub.2006.07.057.

Lampson, M.A., K. Renduchitala, A. Khodjakov, and T.M. Kapoor. 2004. Correcting improper chromosome-spindle attachments during cell division. Nat. Cell Biol. 6:232237. doi:10.1038/ncb1102.

Lan, W., X. Zhang, S.L. Kline-Smith, S.E. Rosasco, G.A. Barrett-Wilt, J. Shabanowitz, D.F. Hunt, C.E. Walczak, and P.T. stukenberg. 2004. Aurora B phosphorylates centromeric MCAK and regulates its localization and microtubule depolymerization activity. Curr. Biol. 14:273-286. doi:10.1016/j.cub.2004.01.055.

Lansbergen, G., and A. Akhmanova. 2006. Microtubule plus end: a hub of cellular activities. Traffic. 7:499-507. doi:10.1111/j.1600-0854.2006.00400.x.

Lee, L., J.S. Tirnauer, J. Li, S.C. Schuyler, J.Y. Liu, and D. Pellman. 2000. Positioning of the mitotic spindle by a cortical-microtubule capture mechanism. Science. 287:22602262.

Li, R., and A.W. Murray. 1991. Feedback control of mitosis in budding yeast. Cell. 66:519-531.

Liu, D., G. Vader, M.J.M. Vromans, M.A. Lampson, and S.M.A. Lens. 2009. Sensing chromosome bi-orientation by spatial separation of aurora B kinase from kinetochore substrates. Science. 323:1350-1353. doi:10.1126/science.1167000.

Liu, X., and M. Winey. 2012. The MPS1 family of protein kinases. Annu. Rev. Biochem. 81:561-585. doi:10.1146/annurev-biochem-061611-090435.

Losada, A., M. Hirano, and T. Hirano. 2002. Cohesin release is required for sister chromatid resolution, but not for condensin-mediated compaction, at the onset of mitosis. Genes \& Development. 16:3004-3016. doi:10.1101/gad.249202.

Maciejowski, J., K.A. George, M.-E. Terret, C. Zhang, K.M. Shokat, and P.V. Jallepalli. 2010. Mps1 directs the assembly of Cdc20 inhibitory complexes during interphase and mitosis to control M phase timing and spindle checkpoint signaling. J. Cell Biol. 190:89100. doi: $10.1083 /$ jcb.201001050.

Mackay, A.M., A.M. Ainsztein, D.M. Eckley, and W.C. Earnshaw. 1998. A dominant mutant of inner centromere protein (INCENP), a chromosomal protein, disrupts prometaphase congression and cytokinesis. J. Cell Biol. 140:991-1002.

Maiato, H., J. DeLuca, E.D. Salmon, and W.C. Earnshaw. 2004. The dynamic kinetochore-microtubule interface. J. Cell. Sci. 117:5461-5477. doi:10.1242/jcs.01536. 
Matson, D.R., P.B. Demirel, P.T. stukenberg, and D.J. Burke. 2012. A conserved role for COMA/CENP-H/I/N kinetochore proteins in the spindle checkpoint. Genes \& Development. 26:542-547. doi:10.1101/gad.184184.111.

Mayer, T.U., T.M. Kapoor, S.J. Haggarty, R.W. King, S.L. Schreiber, and T.J. Mitchison. 1999. Small molecule inhibitor of mitotic spindle bipolarity identified in a phenotypebased screen. Science. 286:971-974.

Miller, S.A., M.L. Johnson, and P.T. stukenberg. 2008. Kinetochore attachments require an interaction between unstructured tails on microtubules and Ndc80(Hec1). Curr. Biol. 18:1785-1791. doi:10.1016/j.cub.2008.11.007.

Minoshima, Y., T. Kawashima, K. Hirose, Y. Tonozuka, A. Kawajiri, Y.C. Bao, X. Deng, M. Tatsuka, S. Narumiya, W.S. May, T. Nosaka, K. Semba, T. Inoue, T. Satoh, M. Inagaki, and T. Kitamura. 2003. Phosphorylation by aurora B converts MgcRacGAP to a RhoGAP during cytokinesis. Dev Cell. 4:549-560.

Mishra, R.K., P. Chakraborty, A. Arnaoutov, B.M.A. Fontoura, and M. Dasso. 2010. The Nup107-160 complex and gamma-TuRC regulate microtubule polymerization at kinetochores. Nat. Cell Biol. 12:164-169. doi:10.1038/ncb2016.

Mitchison, T.J., and M.W. Kirschner. 1985. Properties of the kinetochore in vitro. I. Microtubule nucleation and tubulin binding. J. Cell Biol. 101:755-765.

Morrison, E.E., B.N. Wardleworth, J.M. Askham, A.F. Markham, and D.M. Meredith. 1998. EB1, a protein which interacts with the APC tumour suppressor, is associated with the microtubule cytoskeleton throughout the cell cycle. Oncogene. 17:3471-3477. doi:10.1038/sj.onc. 1202247.

Murnion, M.E., R.R. Adams, D.M. Callister, C.D. Allis, W.C. Earnshaw, and J.R. Swedlow. 2001. Chromatin-associated protein phosphatase 1 regulates aurora-B and histone H3 phosphorylation. J. Biol. Chem. 276:26656-26665.

doi:10.1074/jbc.M102288200.

Musacchio, A., and K.G. Hardwick. 2002. The spindle checkpoint: structural insights into dynamic signalling. Nat Rev Mol Cell Biol. 3:731-741. doi:10.1038/nrm929.

Nakajima, Y., A. Cormier, R.G. Tyers, A. Pigula, Y. Peng, D.G. Drubin, and G. Barnes. 2011. Ipl1/Aurora-dependent phosphorylation of Sli15/INCENP regulates CPC-spindle interaction to ensure proper microtubule dynamics. J. Cell Biol. 194:137-153. doi:10.1083/jcb.201009137.

Nakajima, Y., R.G. Tyers, C.C.L. Wong, J.R. Yates, D.G. Drubin, and G. Barnes. 2009. Nbl1p: a Borealin/Dasra/CSC-1-like protein essential for Aurora/Ipl1 complex function and integrity in Saccharomyces cerevisiae. Mol. Biol. Cell. 20:1772-1784.

doi:10.1091/mbc.E08-10-1011.

Nasmyth, K. 2002. Segregating sister genomes: the molecular biology of chromosome 
separation. Science. 297:559-565. doi:10.1126/science.1074757.

Nicklas, R.B., and C.A. Koch. 1969. Chromosome micromanipulation. 3. Spindle fiber tension and the reorientation of mal-oriented chromosomes. J. Cell Biol. 43:40-50.

Nicklas, R.B., J.C. Waters, E.D. Salmon, and S.C. Ward. 2001. Checkpoint signals in grasshopper meiosis are sensitive to microtubule attachment, but tension is still essential. J. Cell. Sci. 114:4173-4183.

Niedzialkowska, E., F. Wang, P.J. Porebski, W. Minor, J.M.G. Higgins, and P.T. stukenberg. 2012. Molecular basis for phosphospecific recognition of histone $\mathrm{H} 3$ tails by Survivin paralogues at inner centromeres. Mol. Biol. Cell. 23:1457-1466. doi:10.1091/mbc.E11-11-0904.

Orjalo, A.V., A. Arnaoutov, Z. Shen, Y. Boyarchuk, S.G. Zeitlin, B. Fontoura, S. Briggs, M. Dasso, and D.J. Forbes. 2006. The Nup107-160 nucleoporin complex is required for correct bipolar spindle assembly. Mol. Biol. Cell. 17:3806-3818. doi:10.1091/mbc.E0511-1061.

Paweletz, N. 2001. Walther Flemming: pioneer of mitosis research. 2. 4 pp.

Platani, M., R. Santarella-Mellwig, M. Posch, R. Walczak, J.R. Swedlow, and I.W. Mattaj. 2009. The Nup107-160 nucleoporin complex promotes mitotic events via control of the localization state of the chromosome passenger complex. Mol. Biol. Cell. 20:52605275. doi:10.1091/mbc.E09-05-0377.

Porter, L.A., and D.J. Donoghue. 2003. Cyclin B1 and CDK1: nuclear localization and upstream regulators. Prog Cell Cycle Res. 5:335-347.

Qian, J., M. Beullens, B. Lesage, and M. Bollen. 2013. Aurora B Defines Its Own Chromosomal Targeting by Opposing the Recruitment of the Phosphatase Scaffold RepoMan. Curr. Biol. doi:10.1016/j.cub.2013.05.017.

Reed, S.I. 2003. Ratchets and clocks: the cell cycle, ubiquitylation and protein turnover. Nat Rev Mol Cell Biol. 4:855-864. doi:10.1038/nrm1246.

Rieder, C.L., A. Schultz, R. Cole, and G. Sluder. 1994. Anaphase onset in vertebrate somatic cells is controlled by a checkpoint that monitors sister kinetochore attachment to the spindle. J. Cell Biol. 127:1301-1310.

Rieder, C.L., R.W. Cole, A. Khodjakov, and G. Sluder. 1995. The checkpoint delaying anaphase in response to chromosome monoorientation is mediated by an inhibitory signal produced by unattached kinetochores. J. Cell Biol. 130:941-948.

Rosasco-Nitcher, S.E., W. Lan, S. Khorasanizadeh, and P.T. stukenberg. 2008. Centromeric Aurora-B activation requires TD-60, microtubules, and substrate priming phosphorylation. Science. 319:469-472. doi:10.1126/science.1148980. 
Ruchaud, S., M. Carmena, and W.C. Earnshaw. 2007. Chromosomal passengers: conducting cell division. Nat Rev Mol Cell Biol. 8:798-812. doi:10.1038/nrm2257.

Salimian, K.J., E.R. Ballister, E.M. Smoak, S. Wood, T. Panchenko, M.A. Lampson, and B.E. Black. 2011. Feedback control in sensing chromosome biorientation by the Aurora B kinase. Curr. Biol. 21:1158-1165. doi:10.1016/j.cub.2011.06.015.

Sampath, S.C., R. Ohi, O. Leismann, A. Salic, A. Pozniakovski, and H. Funabiki. 2004. The chromosomal passenger complex is required for chromatin-induced microtubule stabilization and spindle assembly. Cell. 118:187-202. doi:10.1016/j.cell.2004.06.026.

Santaguida, S., C. Vernieri, F. Villa, A. Ciliberto, and A. Musacchio. 2011. Evidence that Aurora B is implicated in spindle checkpoint signalling independently of error correction. EMBO J. 30:1508-1519. doi:10.1038/emboj.2011.70.

Sasai, K., H. Katayama, D.L. Stenoien, S. Fujii, R. Honda, M. Kimura, Y. Okano, M. Tatsuka, F. Suzuki, E.A. Nigg, W.C. Earnshaw, W.R. Brinkley, and S. Sen. 2004. Aurora- $\mathrm{C}$ kinase is a novel chromosomal passenger protein that can complement AuroraB kinase function in mitotic cells. Cell Motil. Cytoskeleton. 59:249-263. doi: $10.1002 / \mathrm{cm} .20039$.

Saurin, A.T., M.S. van der Waal, R.H. Medema, S.M.A. Lens, and G.J.P.L. Kops. 2011. Aurora B potentiates Mps1 activation to ensure rapid checkpoint establishment at the onset of mitosis. Nat Commun. 2:316. doi:10.1038/ncomms1319.

Schmidt, J.C., H. Arthanari, A. Boeszoermenyi, N.M. Dashkevich, E.M. WilsonKubalek, N. Monnier, M. Markus, M. Oberer, R.A. Milligan, M. Bathe, G. Wagner, E.L. Grishchuk, and I.M. Cheeseman. 2012. The kinetochore-bound Skal complex tracks depolymerizing microtubules and binds to curved protofilaments. Dev Cell. 23:968-980. doi:10.1016/j.devcel.2012.09.012.

Schumacher, J.M., N. Ashcroft, P.J. Donovan, and A. Golden. 1998. A highly conserved centrosomal kinase, AIR-1, is required for accurate cell cycle progression and segregation of developmental factors in Caenorhabditis elegans embryos. Development. 125:4391-4402.

Sessa, F., M. Mapelli, C. Ciferri, C. Tarricone, L.B. Areces, T.R. Schneider, P.T. stukenberg, and A. Musacchio. 2005. Mechanism of Aurora B activation by INCENP and inhibition by hesperadin. Mol Cell. 18:379-391. doi:10.1016/j.molcel.2005.03.031.

Sha, W., J. Moore, K. Chen, A.D. Lassaletta, C.-S. Yi, J.J. Tyson, and J.C. Sible. 2003. Hysteresis drives cell-cycle transitions in Xenopus laevis egg extracts. Proc Natl Acad Sci U S A. 100:975-980. doi:10.1073/pnas.0235349100.

Siegel, L.M., and K.J. Monty. 1966. Determination of molecular weights and frictional ratios of proteins in impure systems by use of gel filtration and density gradient centrifugation. Application to crude preparations of sulfite and hydroxylamine reductases. Biochim. Biophys. Acta. 112:346-362. 
Söderberg, O., M. Gullberg, M. Jarvius, K. Ridderstråle, K.-J. Leuchowius, J. Jarvius, K. Wester, P. Hydbring, F. Bahram, L.-G. Larsson, and U. Landegren. 2006. Direct observation of individual endogenous protein complexes in situ by proximity ligation. Nat. Methods. 3:995-1000. doi:10.1038/nmeth947.

Stucke, V.M., C. Baumann, and E.A. Nigg. 2004. Kinetochore localization and microtubule interaction of the human spindle checkpoint kinase Mps1. Chromosoma. 113:1-15. doi:10.1007/s00412-004-0288-2.

Stucke, V.M., H.H.W. Silljé, L. Arnaud, and E.A. Nigg. 2002. Human Mps1 kinase is required for the spindle assembly checkpoint but not for centrosome duplication. $E M B O$ J. 21:1723-1732. doi:10.1093/emboj/21.7.1723.

Su, L.K., M. Burrell, D.E. Hill, J. Gyuris, R. Brent, R. Wiltshire, J. Trent, B. Vogelstein, and K.W. Kinzler. 1995. APC binds to the novel protein EB1. Cancer Res. 55:29722977.

Sun, L., J. Gao, X. Dong, M. Liu, D. Li, X. Shi, J.-T. Dong, X. Lu, C. Liu, and J. Zhou. 2008. EB1 promotes Aurora-B kinase activity through blocking its inactivation by protein phosphatase 2A. Proc Natl Acad Sci U S A. 105:7153-7158. doi:10.1073/pnas.0710018105.

Tanaka, T.U., M.J.R. Stark, and K. Tanaka. 2005. Kinetochore capture and bi-orientation on the mitotic spindle. Nat Rev Mol Cell Biol. 6:929-942. doi:10.1038/nrm1764.

Tanaka, T.U., N. Rachidi, C. Janke, G. Pereira, M. Galova, E. Schiebel, M.J.R. Stark, and K. Nasmyth. 2002. Evidence that the Ipl1-Sli15 (Aurora kinase-INCENP) complex promotes chromosome bi-orientation by altering kinetochore-spindle pole connections. Cell. 108:317-329.

Terada, Y. 2001. Role of chromosomal passenger complex in chromosome segregation and cytokinesis. Cell Struct. Funct. 26:653-657.

Terada, Y., M. Tatsuka, F. Suzuki, Y. Yasuda, S. Fujita, and M. Otsu. 1998. AIM-1: a mammalian midbody-associated protein required for cytokinesis. EMBO J. 17:667-676. doi:10.1093/emboj/17.3.667.

Tighe, A., O. Staples, and S. Taylor. 2008. Mps1 kinase activity restrains anaphase during an unperturbed mitosis and targets Mad2 to kinetochores. J. Cell Biol. 181:893901. doi: $10.1083 /$ jcb.200712028.

Tirnauer, J.S., J.C. Canman, E.D. Salmon, and T.J. Mitchison. 2002. EB1 targets to kinetochores with attached, polymerizing microtubules. Mol. Biol. Cell. 13:4308-4316. doi:10.1091/mbc.E02-04-0236.

Tsai, M.-Y., C. Wiese, K. Cao, O. Martin, P. Donovan, J. Ruderman, C. Prigent, and Y. Zheng. 2003. A Ran signalling pathway mediated by the mitotic kinase Aurora A in spindle assembly. Nat. Cell Biol. 5:242-248. doi:10.1038/ncb936. 
Tseng, B.S., L. Tan, T.M. Kapoor, and H. Funabiki. 2010. Dual detection of chromosomes and microtubules by the chromosomal passenger complex drives spindle assembly. Dev Cell. 18:903-912. doi:10.1016/j.devcel.2010.05.018.

Tsukahara, T., Y. Tanno, and Y. Watanabe. 2010. Phosphorylation of the CPC by Cdk1 promotes chromosome bi-orientation. Nature. 467:719-723. doi:10.1038/nature09390.

Tulu, U.S., C. Fagerstrom, N.P. Ferenz, and P. Wadsworth. 2006. Molecular requirements for kinetochore-associated microtubule formation in mammalian cells. Curr. Biol. 16:536-541. doi:10.1016/j.cub.2006.01.060.

Vader, G., J.J.W. Kauw, R.H. Medema, and S.M.A. Lens. 2006. Survivin mediates targeting of the chromosomal passenger complex to the centromere and midbody. EMBO Rep. 7:85-92. doi:10.1038/sj.embor.7400562.

Vagnarelli, P., and W.C. Earnshaw. 2004. Chromosomal passengers: the fourdimensional regulation of mitotic events. Chromosoma. 113:211-222. doi:10.1007/s00412-004-0307-3.

Vagnarelli, P., S. Ribeiro, L. Sennels, L. Sanchez-Pulido, F. de Lima Alves, T. Verheyen, D.A. Kelly, C.P. Ponting, J. Rappsilber, and W.C. Earnshaw. 2011. Repo-Man coordinates chromosomal reorganization with nuclear envelope reassembly during mitotic exit. Dev Cell. 21:328-342. doi:10.1016/j.devcel.2011.06.020.

van der Waal, M.S., A.T. Saurin, M.J.M. Vromans, M. Vleugel, C. Wurzenberger, D.W. Gerlich, R.H. Medema, G.J.P.L. Kops, and S.M.A. Lens. 2012. Mps1 promotes rapid centromere accumulation of Aurora B. EMBO Rep. doi:10.1038/embor.2012.93.

Varmark, H. 2004. Functional role of centrosomes in spindle assembly and organization. J. Cell. Biochem. 91:904-914. doi:10.1002/jcb.20013.

Vong, Q.P., K. Cao, H.Y. Li, P.A. Iglesias, and Y. Zheng. 2005. Chromosome alignment and segregation regulated by ubiquitination of survivin. Science. 310:1499-1504. doi:10.1126/science. 1120160 .

Wang, E., E.R. Ballister, and M.A. Lampson. 2011a. Aurora B dynamics at centromeres create a diffusion-based phosphorylation gradient. J. Cell Biol. 194:539-549. doi: $10.1083 /$ jcb. 201103044 .

Wang, F., J. Dai, J.R. Daum, E. Niedzialkowska, B. Banerjee, P.T. stukenberg, G.J. Gorbsky, and J.M.G. Higgins. 2010. Histone H3 Thr-3 phosphorylation by Haspin positions Aurora B at centromeres in mitosis. Science. 330:231-235. doi:10.1126/science.1189435.

Wang, F., N.P. Ulyanova, J.R. Daum, D. Patnaik, A.V. Kateneva, G.J. Gorbsky, and J.M.G. Higgins. 2012. Haspin inhibitors reveal centromeric functions of Aurora B in chromosome segregation. J. Cell Biol. 199:251-268. doi:10.1083/jcb.201205106. 
Wang, F., N.P. Ulyanova, M.S. van der Waal, D. Patnaik, S.M.A. Lens, and J.M.G. Higgins. 2011b. A positive feedback loop involving Haspin and Aurora B promotes CPC accumulation at centromeres in mitosis. Curr. Biol. 21:1061-1069.

doi:10.1016/j.cub.2011.05.016.

Weiss, E., and M. Winey. 1996. The Saccharomyces cerevisiae spindle pole body duplication gene MPS1 is part of a mitotic checkpoint. J. Cell Biol. 132:111-123.

Welburn, J.P.I., M. Vleugel, D. Liu, J.R. Yates, M.A. Lampson, T. Fukagawa, and I.M. Cheeseman. 2010. Aurora B phosphorylates spatially distinct targets to differentially regulate the kinetochore-microtubule interface. Mol Cell. 38:383-392. doi:10.1016/j.molcel.2010.02.034.

Wheatley, S.P., A. Carvalho, P. Vagnarelli, and W.C. Earnshaw. 2001a. INCENP is required for proper targeting of Survivin to the centromeres and the anaphase spindle during mitosis. Curr. Biol. 11:886-890.

Wheatley, S.P., A.J. Henzing, H. Dodson, W. Khaled, and W.C. Earnshaw. 2004. Aurora-B phosphorylation in vitro identifies a residue of survivin that is essential for its localization and binding to inner centromere protein (INCENP) in vivo. J. Biol. Chem. 279:5655-5660. doi:10.1074/jbc.M311299200.

Wheatley, S.P., S.E. Kandels-Lewis, R.R. Adams, A.M. Ainsztein, and W.C. Earnshaw. 2001b. INCENP binds directly to tubulin and requires dynamic microtubules to target to the cleavage furrow. Exp. Cell Res. 262:122-127. doi:10.1006/excr.2000.5088.

Winey, M., L. Goetsch, P. Baum, and B. Byers. 1991. MPS1 and MPS2: novel yeast genes defining distinct steps of spindle pole body duplication. J. Cell Biol. 114:745-754.

Wordeman, L., and T.J. Mitchison. 1995. Identification and partial characterization of mitotic centromere-associated kinesin, a kinesin-related protein that associates with centromeres during mitosis. J. Cell Biol. 128:95-104.

Xu, Z., P. Vagnarelli, H. Ogawa, K. Samejima, and W.C. Earnshaw. 2010. Gradient of increasing Aurora B kinase activity is required for cells to execute mitosis. Journal of Biological Chemistry. 285:40163-40170. doi:10.1074/jbc.M110.181545.

Yamagishi, Y., T. Honda, Y. Tanno, and Y. Watanabe. 2010. Two histone marks establish the inner centromere and chromosome bi-orientation. Science. 330:239-243. doi:10.1126/science. 1194498 .

Yan, X., L. Cao, Q. Li, Y. Wu, H. Zhang, H. Saiyin, X. Liu, X. Zhang, Q. Shi, and L. Yu. 2005. Aurora $\mathrm{C}$ is directly associated with Survivin and required for cytokinesis. Genes Cells. 10:617-626. doi:10.1111/j.1365-2443.2005.00863.x.

Yokoyama, T., H. Goto, I. Izawa, H. Mizutani, and M. Inagaki. 2005. Aurora-B and Rhokinase/ROCK, the two cleavage furrow kinases, independently regulate the progression of cytokinesis: possible existence of a novel cleavage furrow kinase phosphorylates 
ezrin/radixin/moesin (ERM). Genes Cells. 10:127-137. doi:10.1111/j.13652443.2005.00824.x.

Zhang, X., W. Lan, S.C. Ems-McClung, P.T. stukenberg, and C.E. Walczak. 2007. Aurora B phosphorylates multiple sites on mitotic centromere-associated kinesin to spatially and temporally regulate its function. Mol. Biol. Cell. 18:3264-3276. doi:10.1091/mbc.E07-01-0086.

Zimniak, T., K. Stengl, K. Mechtler, and S. Westermann. 2009. Phosphoregulation of the budding yeast EB1 homologue Bimlp by Aurora/Ipl1p. J. Cell Biol. 186:379-391. doi: $10.1083 /$ jcb. 200901036 .

Zimniak, T., V. Fitz, H. Zhou, F. Lampert, S. Opravil, K. Mechtler, P. Stolt-Bergner, and S. Westermann. 2012. Spatiotemporal regulation of Ipl1/Aurora activity by direct Cdk1 phosphorylation. Curr. Biol. 22:787-793. doi:10.1016/j.cub.2012.03.007. 


\section{APPENDIX}




\section{Cell Lines}

\begin{tabular}{|c|c|c|c|}
\hline Name & Vector & Cell type & Promoter \\
\hline DLAP-hEB1(K89E) & $\begin{array}{c}\text { pCDNA5/FRT-LAP- } \\
\text { hEB1(K89E)-EGFP }\end{array}$ & HeLa-TRex & CMV \\
\hline DLAP-hEB1(siRes) & $\begin{array}{c}\text { pCDNA5/FRT/LAP- } \\
\text { hEB1(siRes) }\end{array}$ & HeLa-TRex & CMV \\
\hline $\begin{array}{c}\text { DLAP- } \\
\text { hEB1(K89E)(siRes) }\end{array}$ & $\begin{array}{c}\text { pCDNA5/FRT/LAP- } \\
\text { hEB1(K89E)(siRes) }\end{array}$ & HeLa-TRex & CMV \\
\hline
\end{tabular}


pET28b-xINCENP-xAuroraB-(6-His)

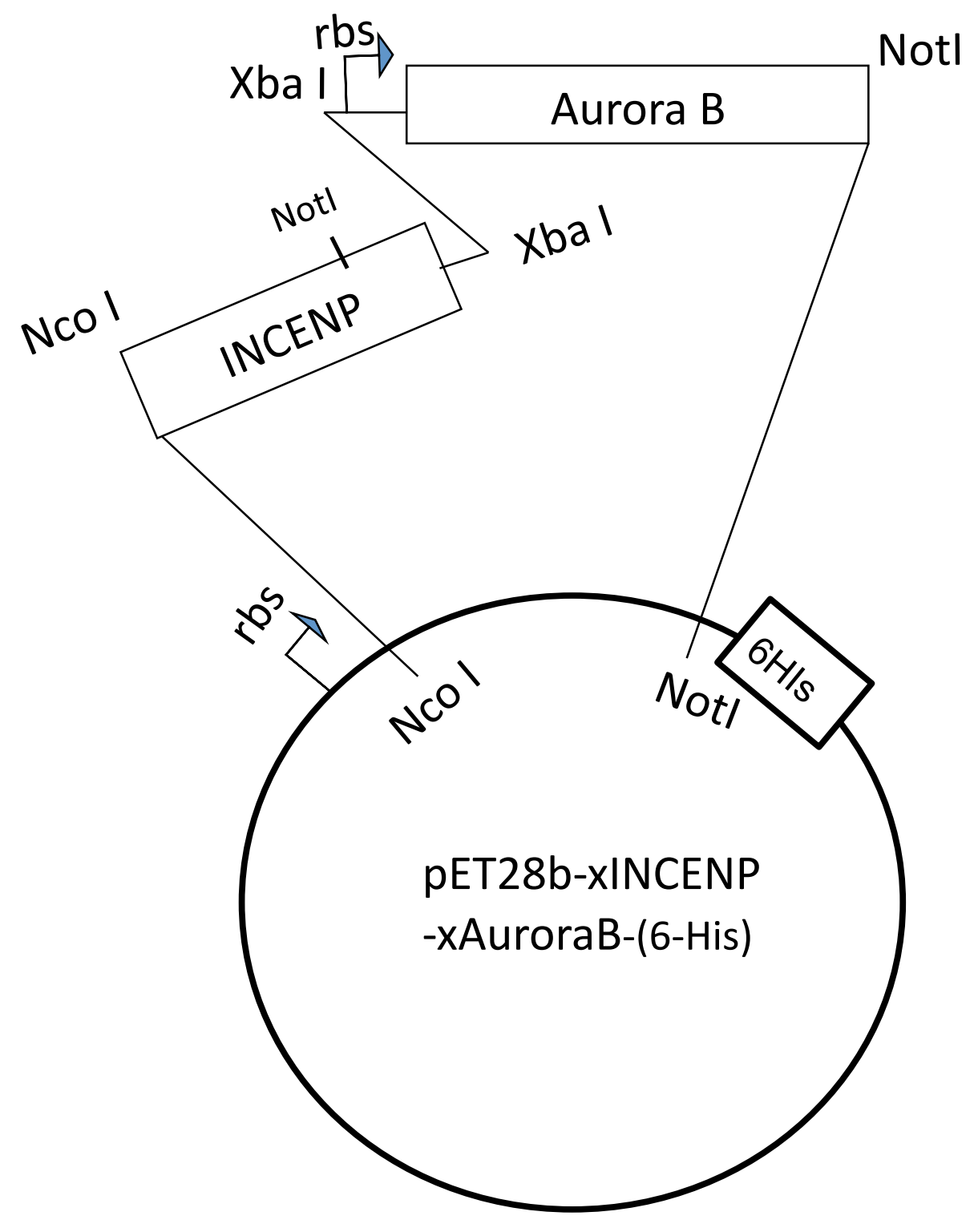


pET15b-xSurvivin-xDasraA (no Tag)

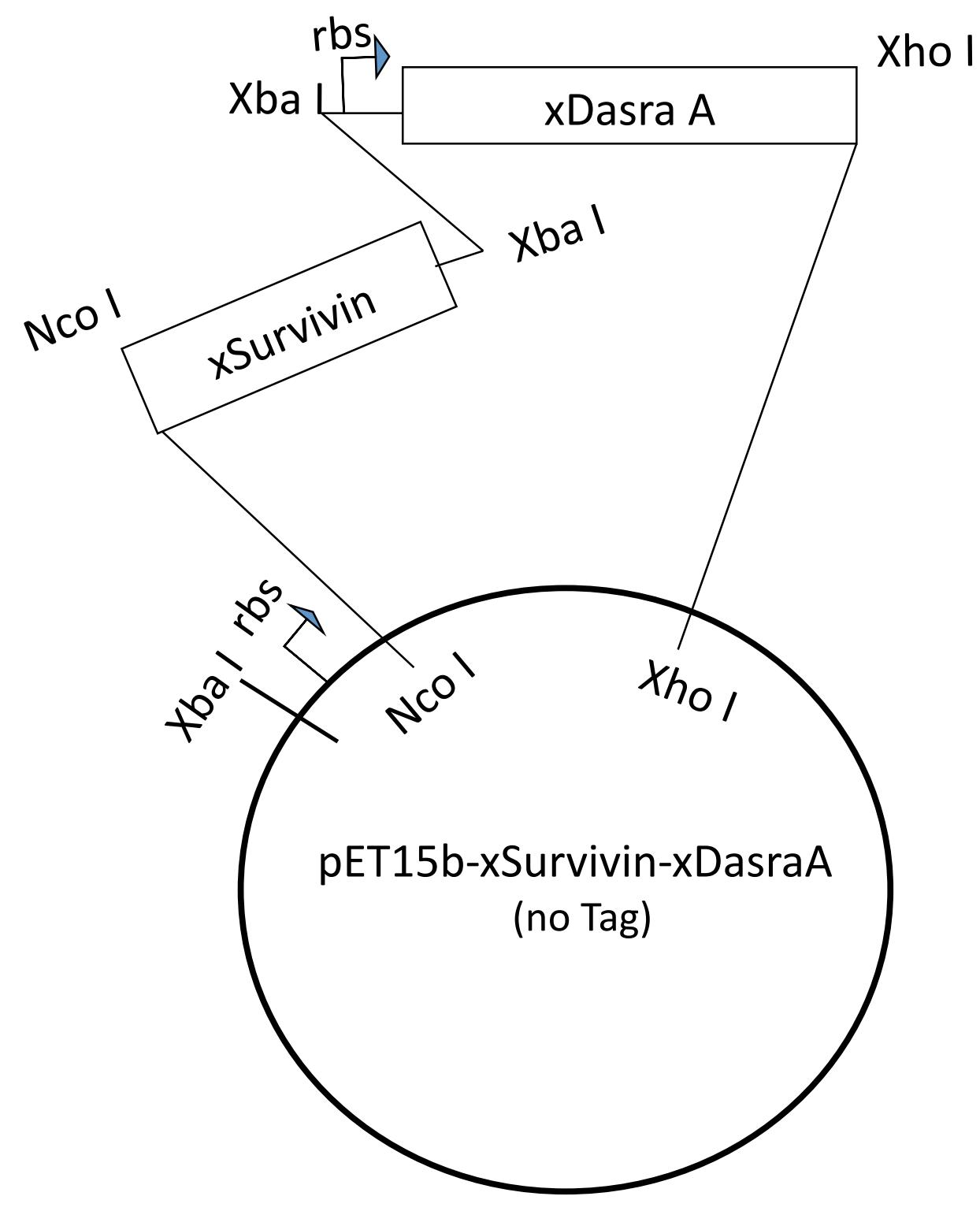


pET28a-xDasraA (S112A)

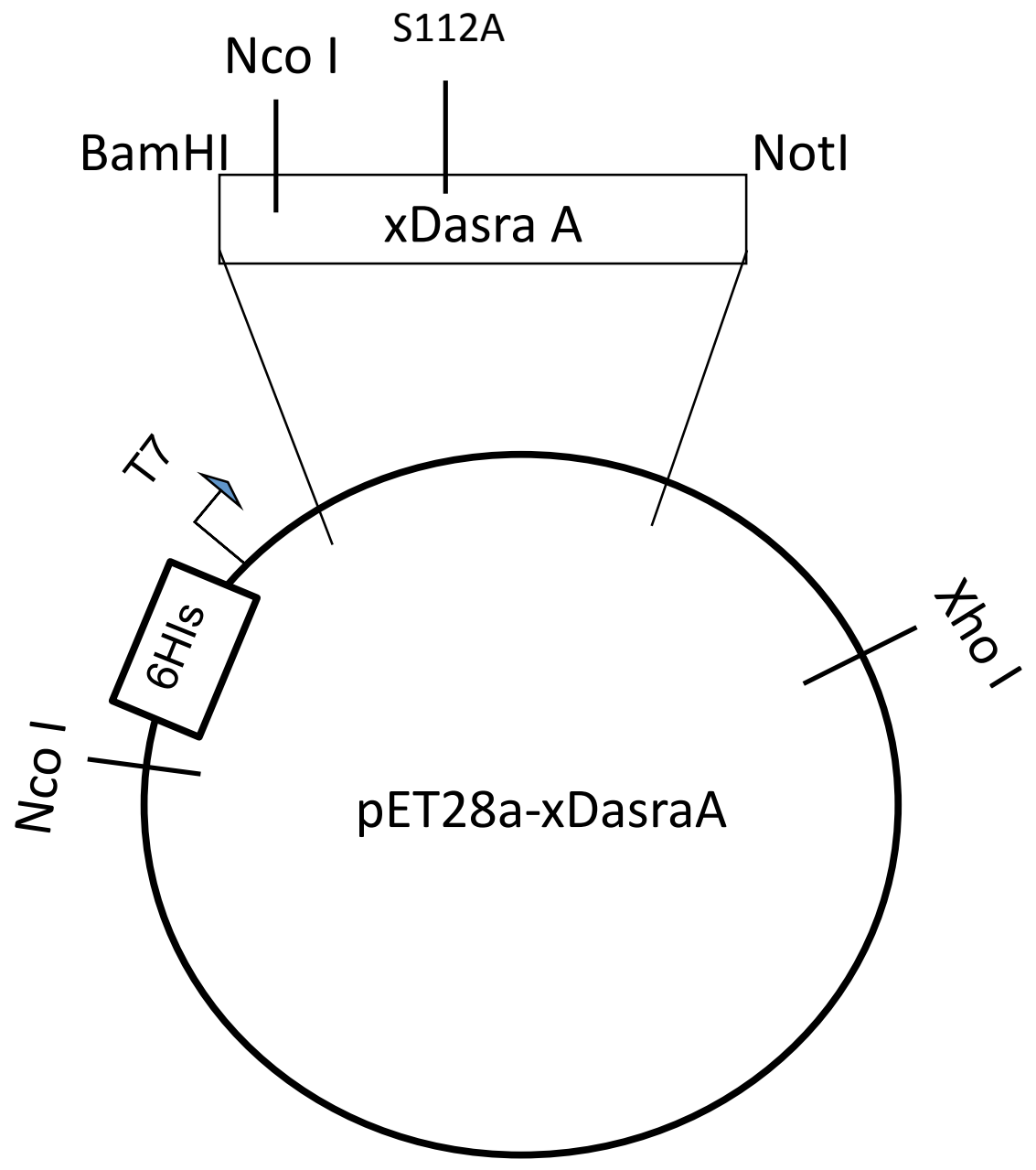


pET28a-xDasraA (S181A)

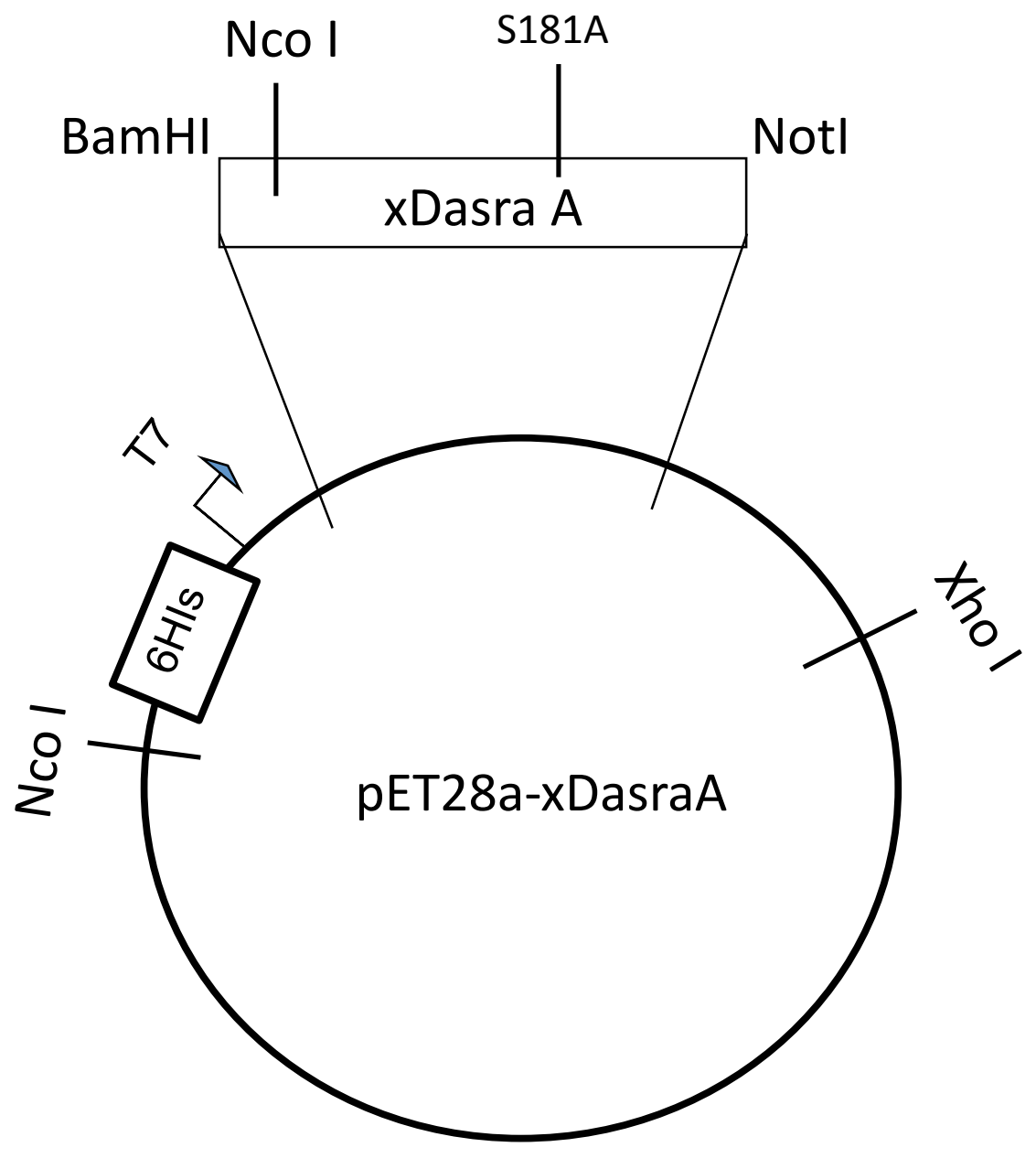


pET28a-xDasraA (S112A, S181A)

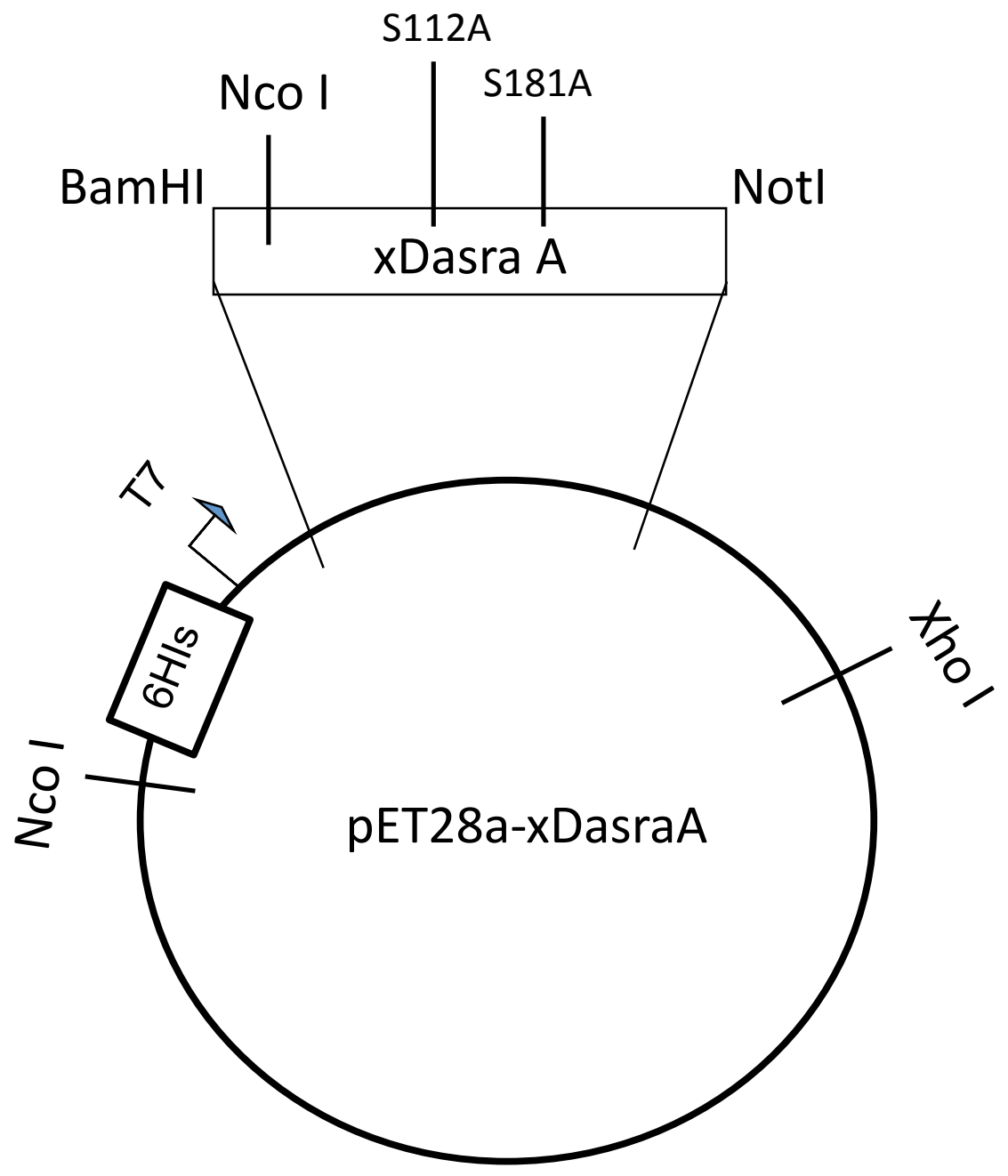


pGEX6P2 FL-AuroraB:INCENP-IN-Box(790-856)

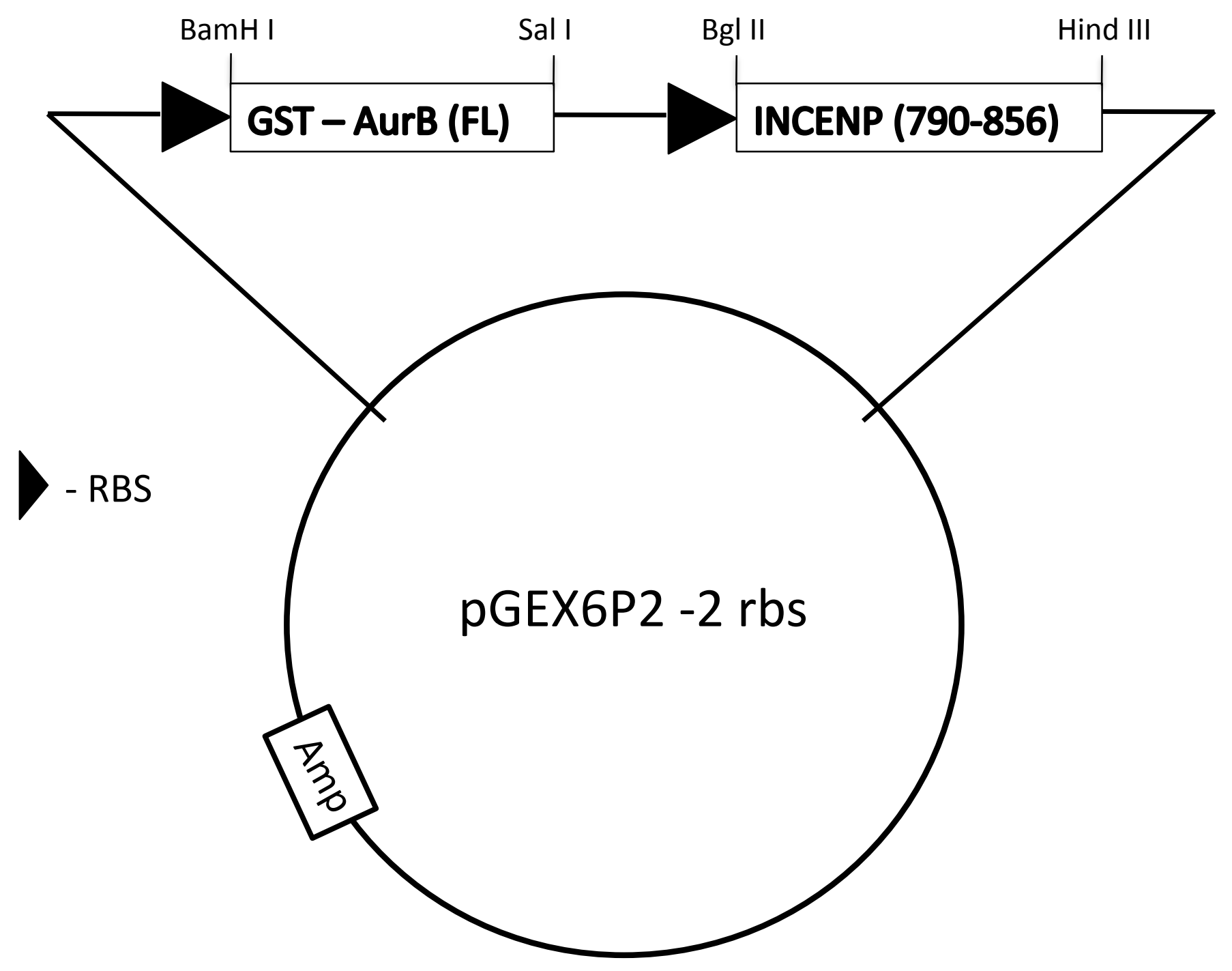


pGEX6P2 FL-AuroraB:INCENP-IN-Box(790-847)

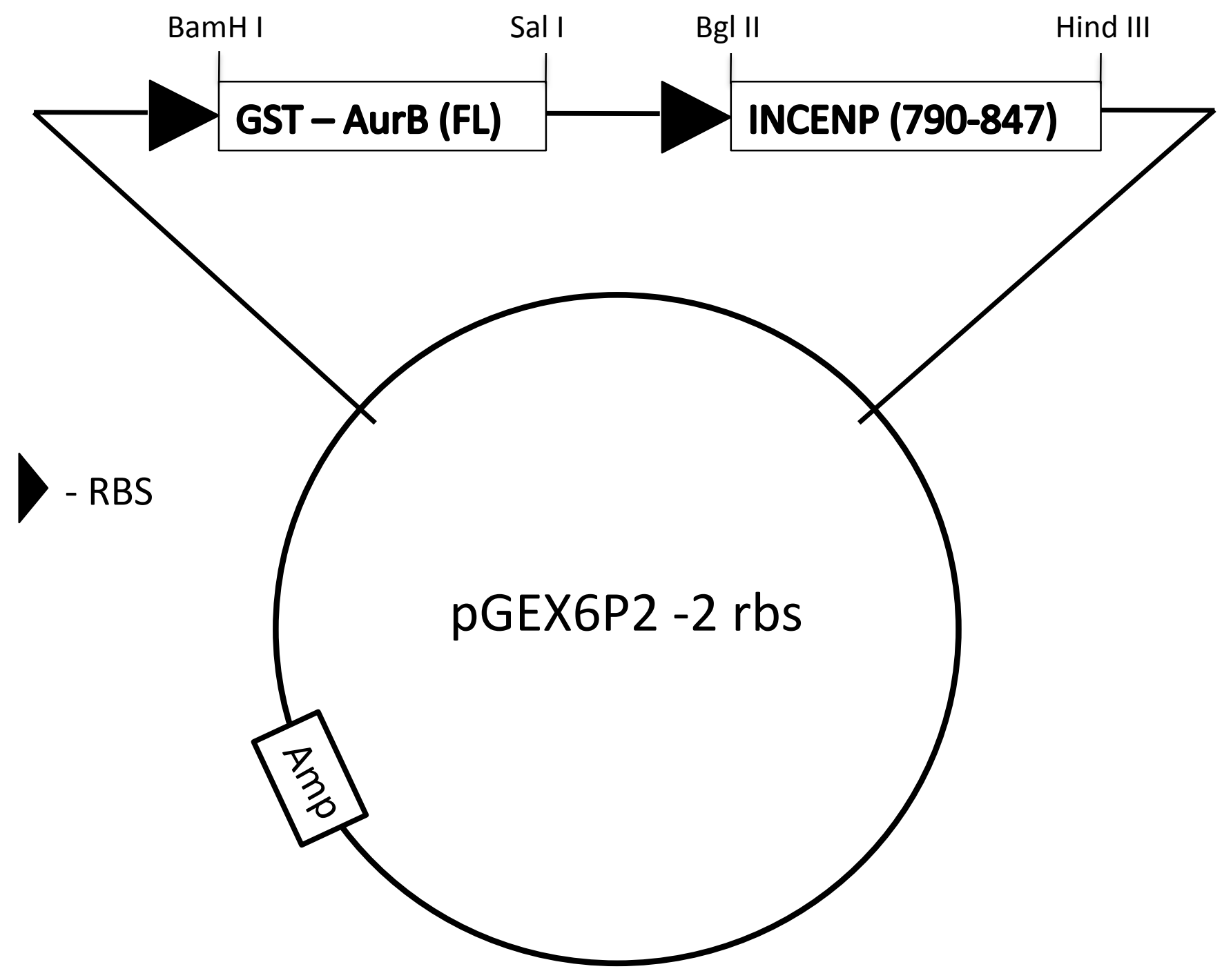


pMCSG7-6His-AuroraB:INCENP-IN-Box(790-856)

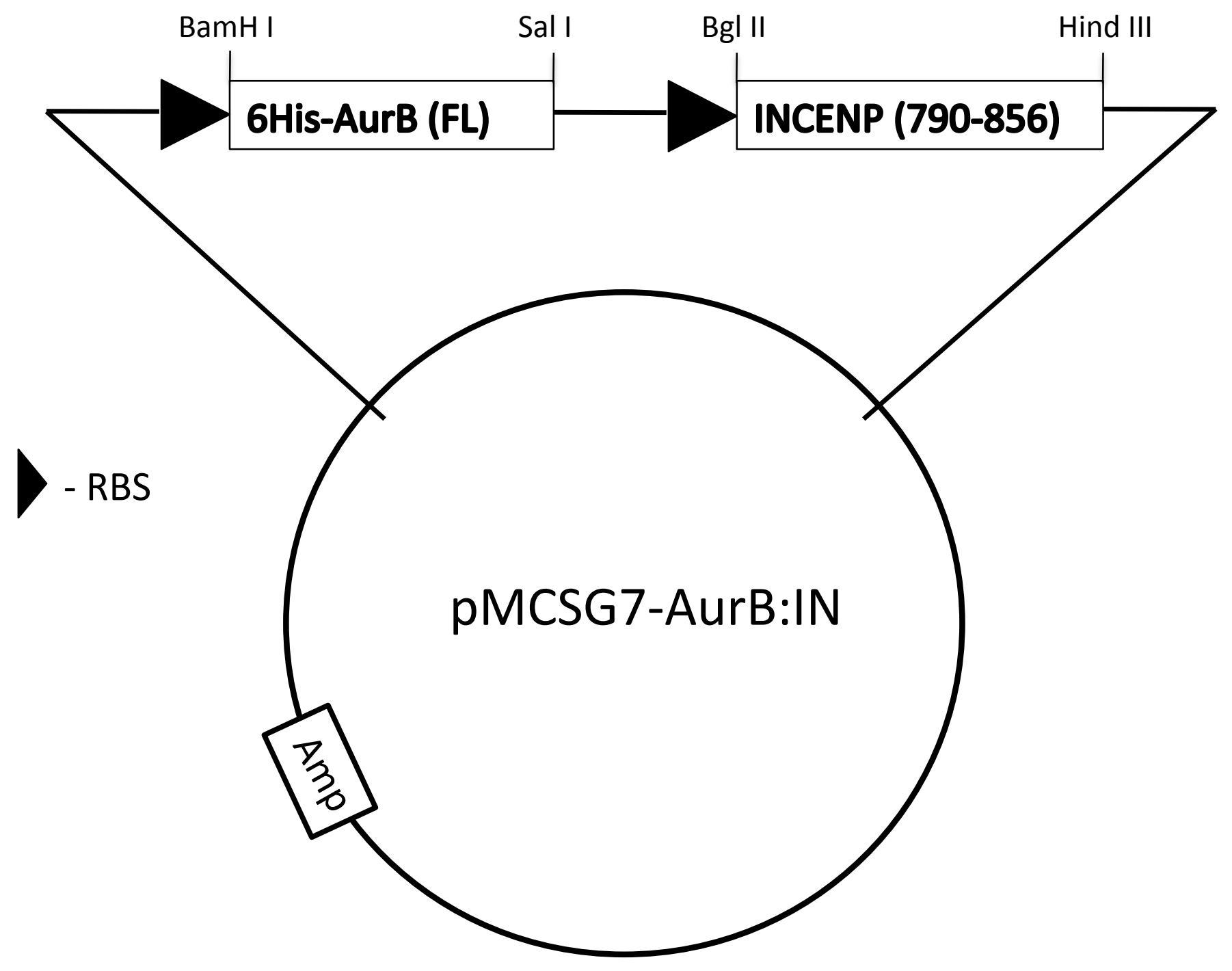


pMCSG7-6His-AuroraB:INCENP-IN-Box(790-847)

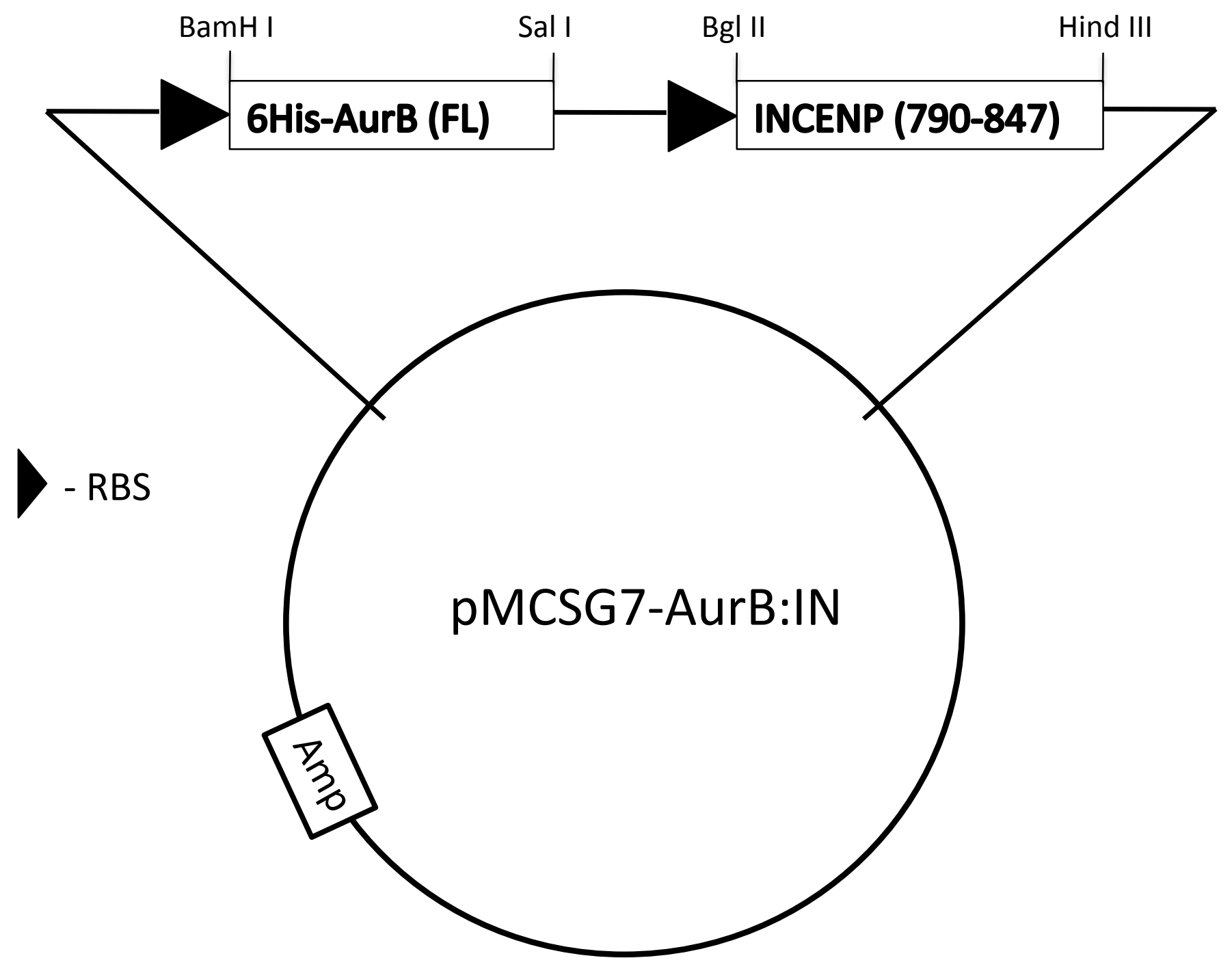




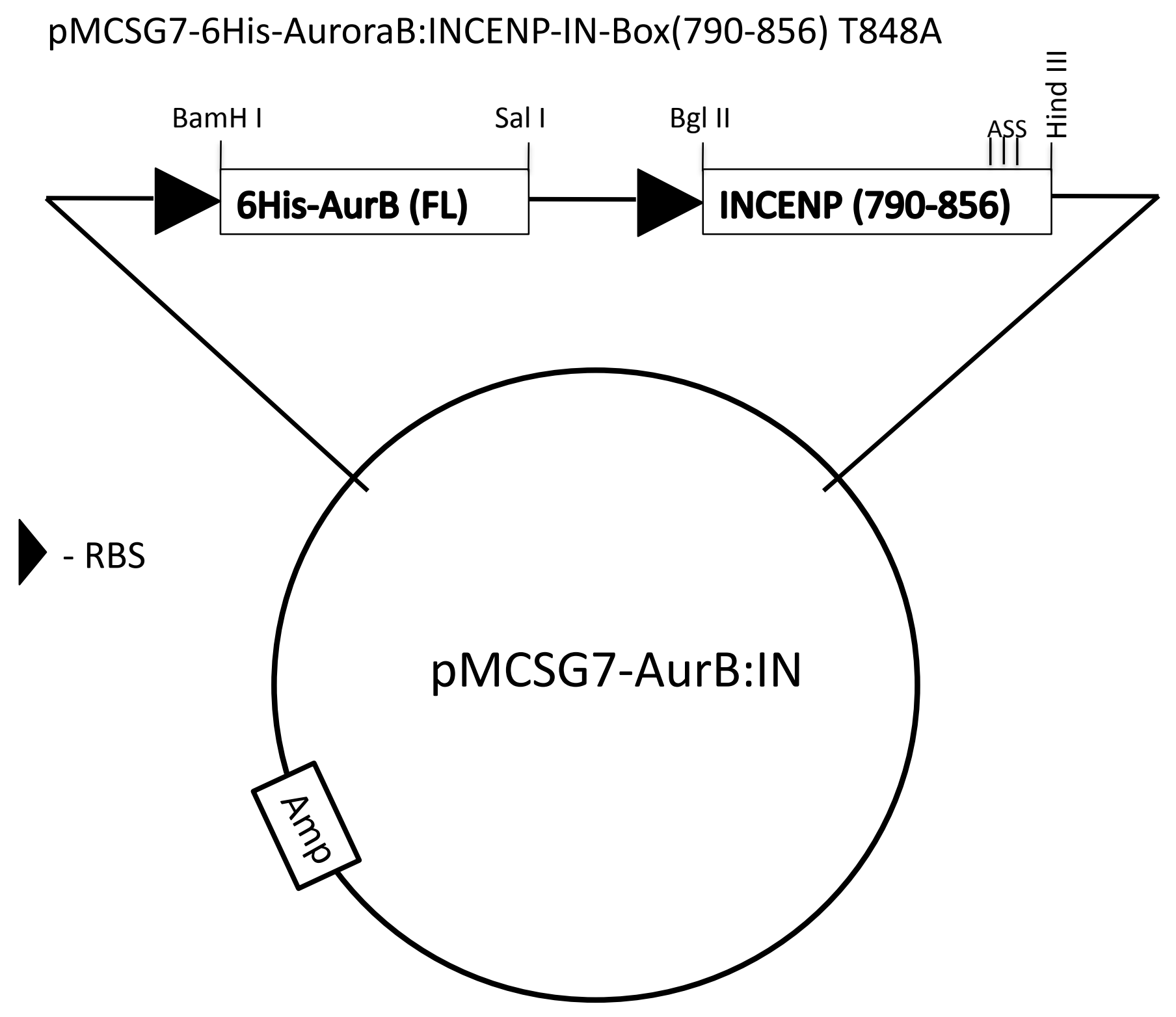




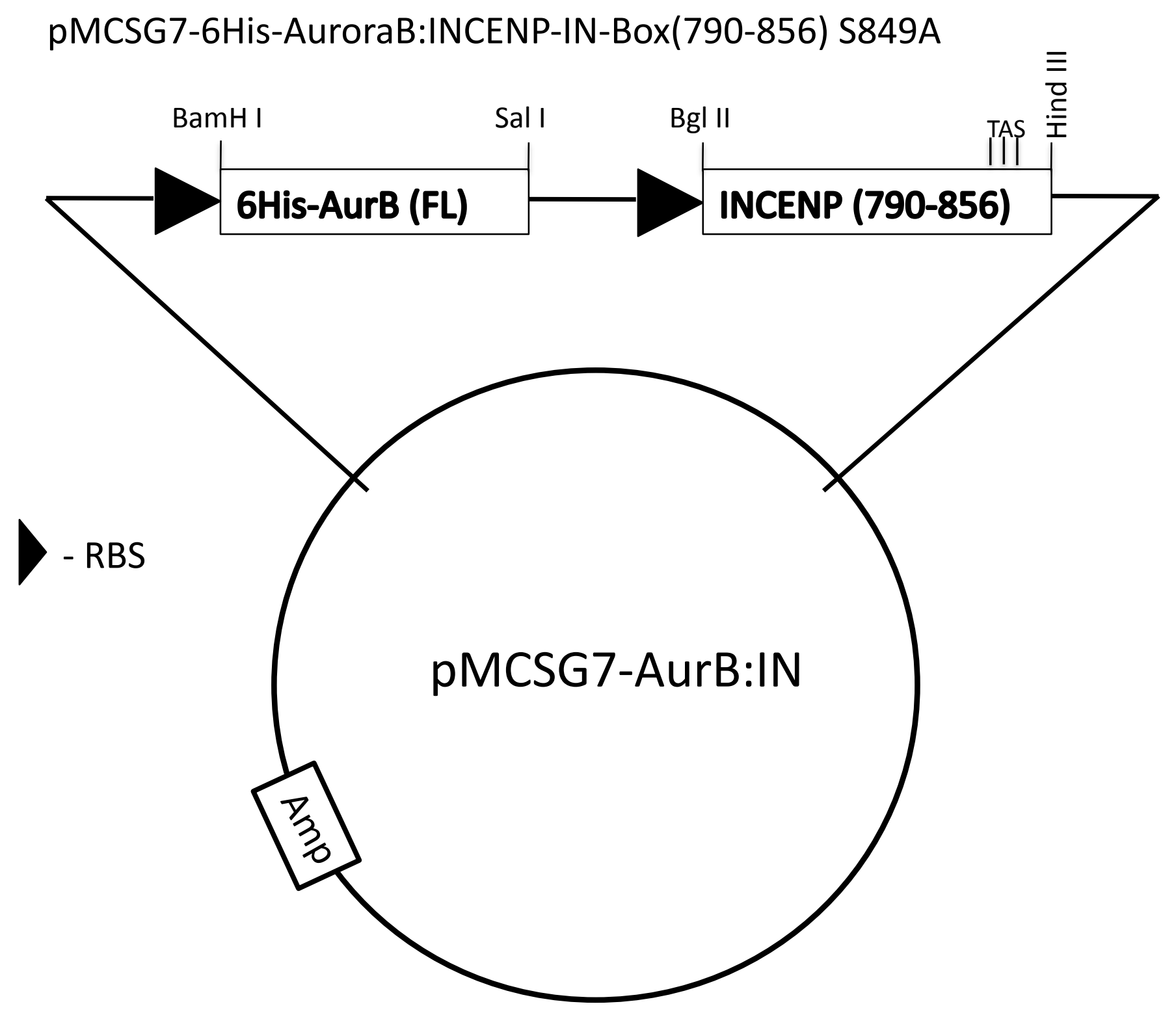




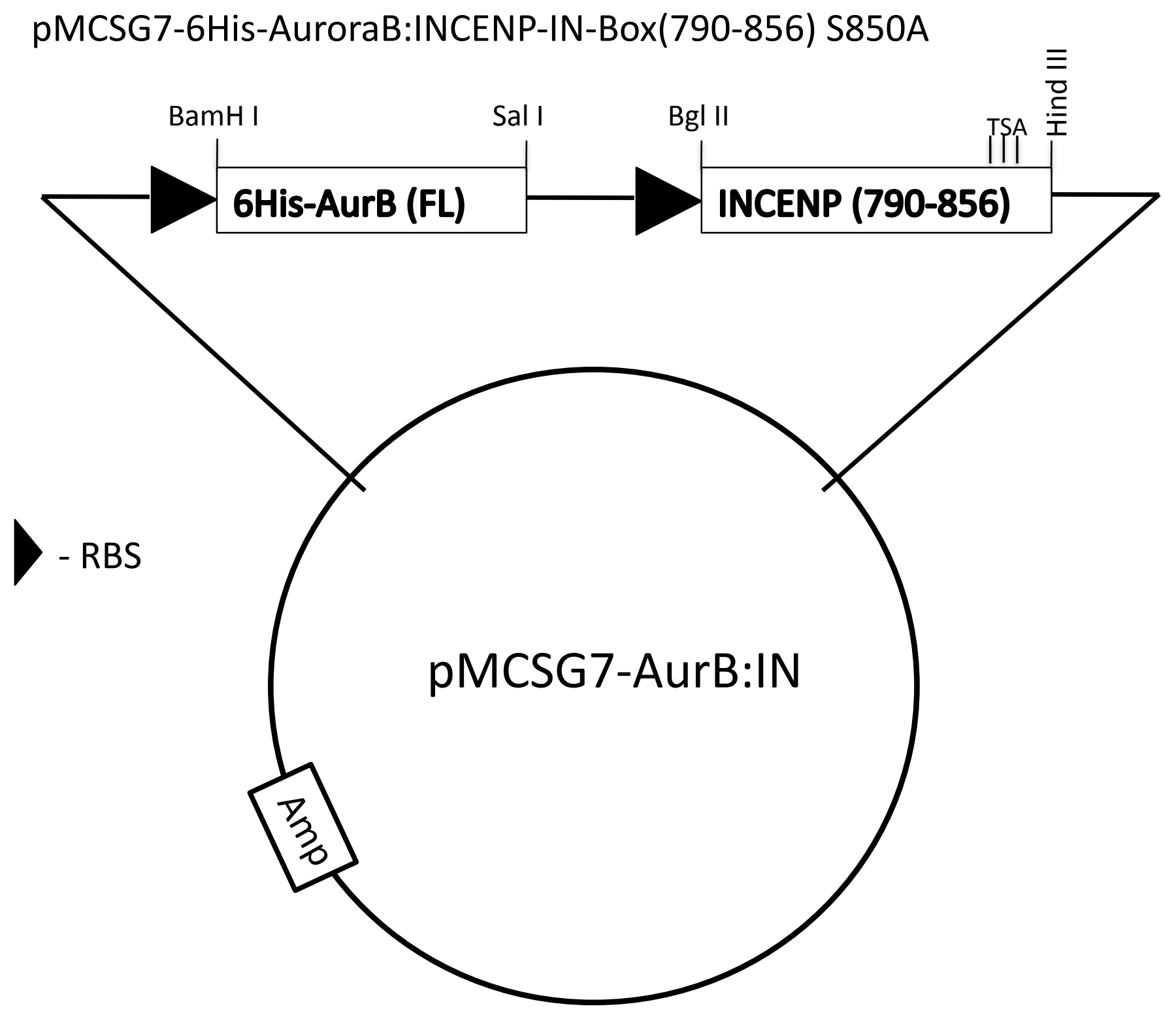




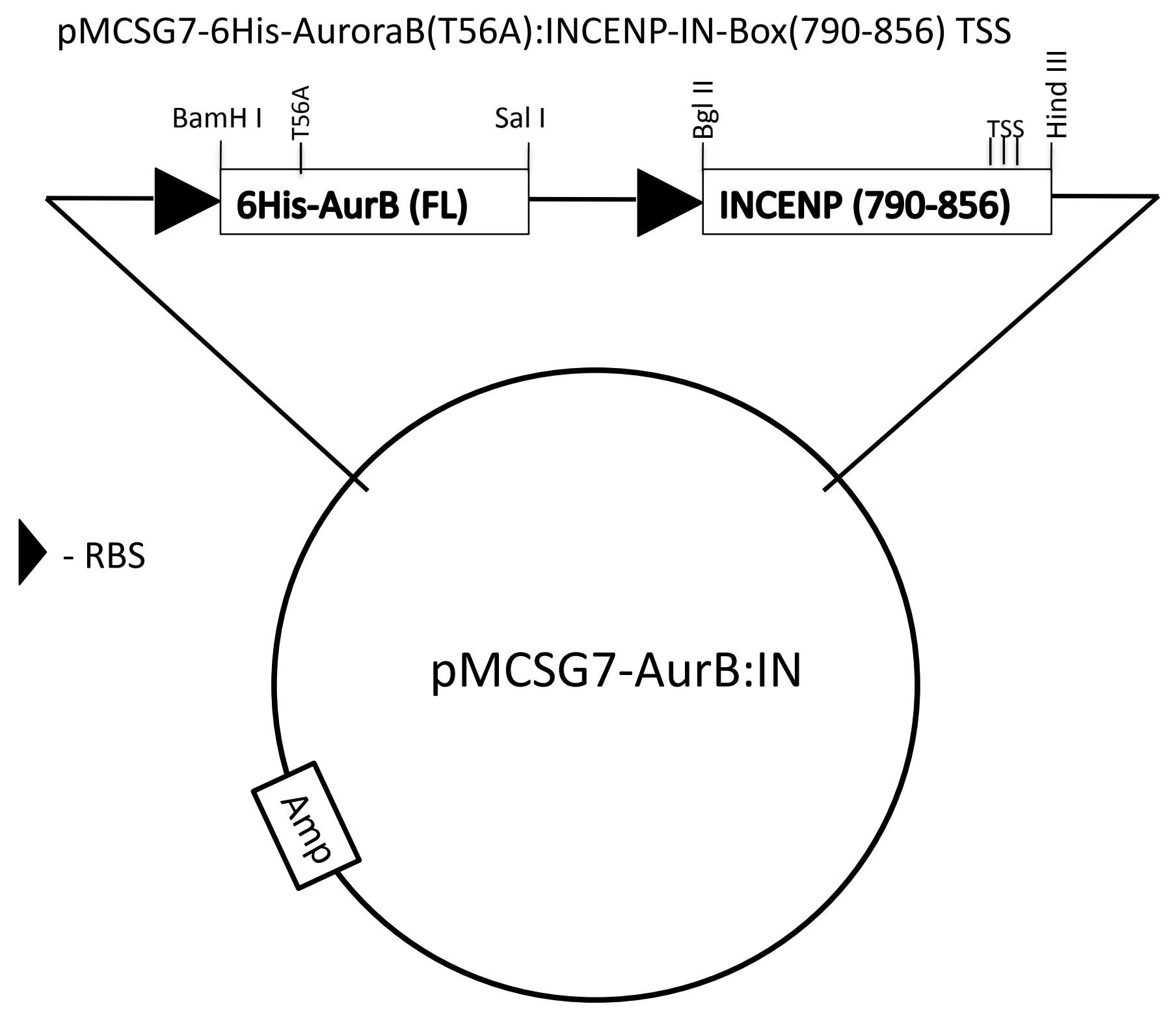




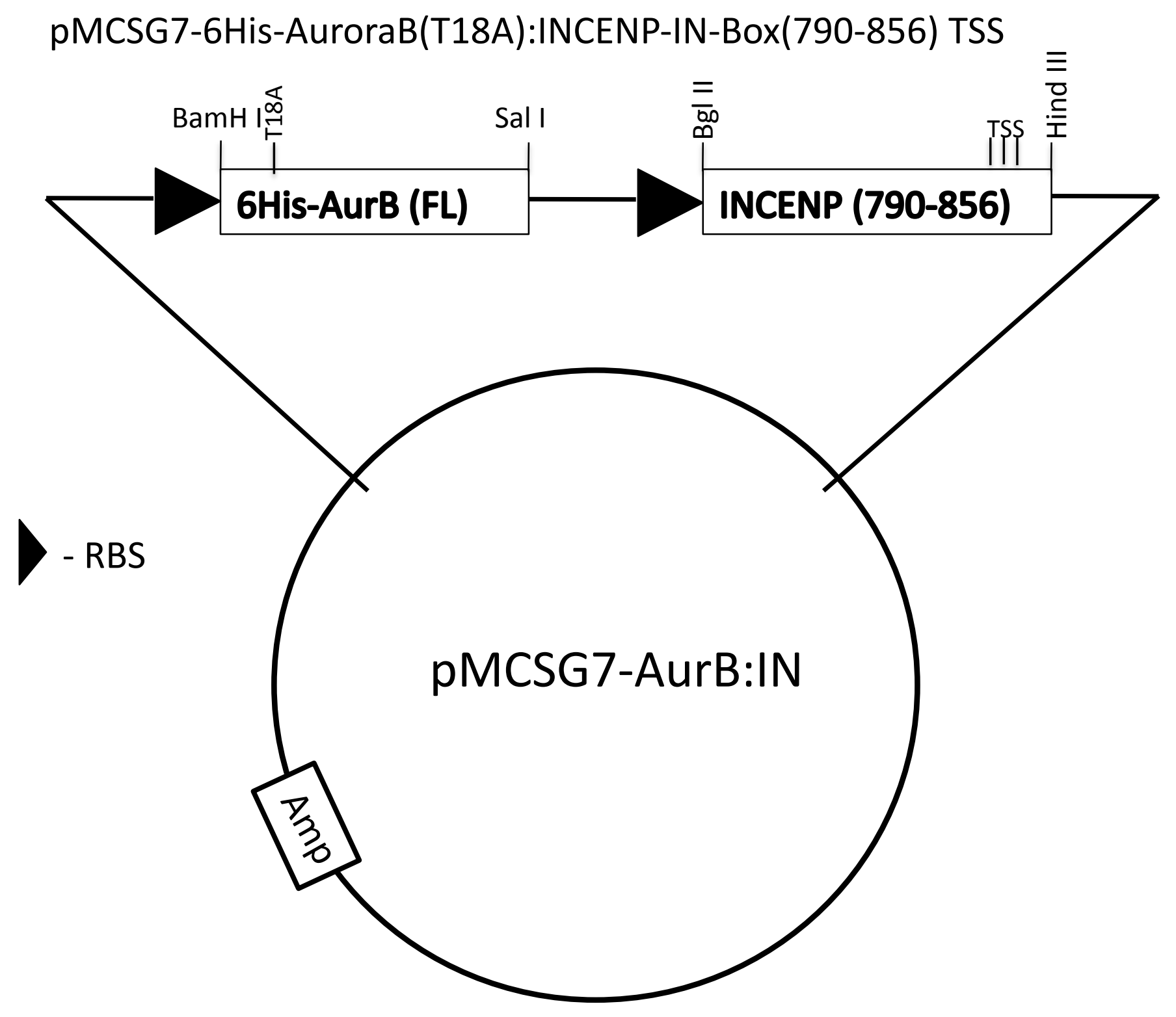


pMCSG7-6His-AuroraB(T17A,T18A):INCENP-IN-Box(790-856)

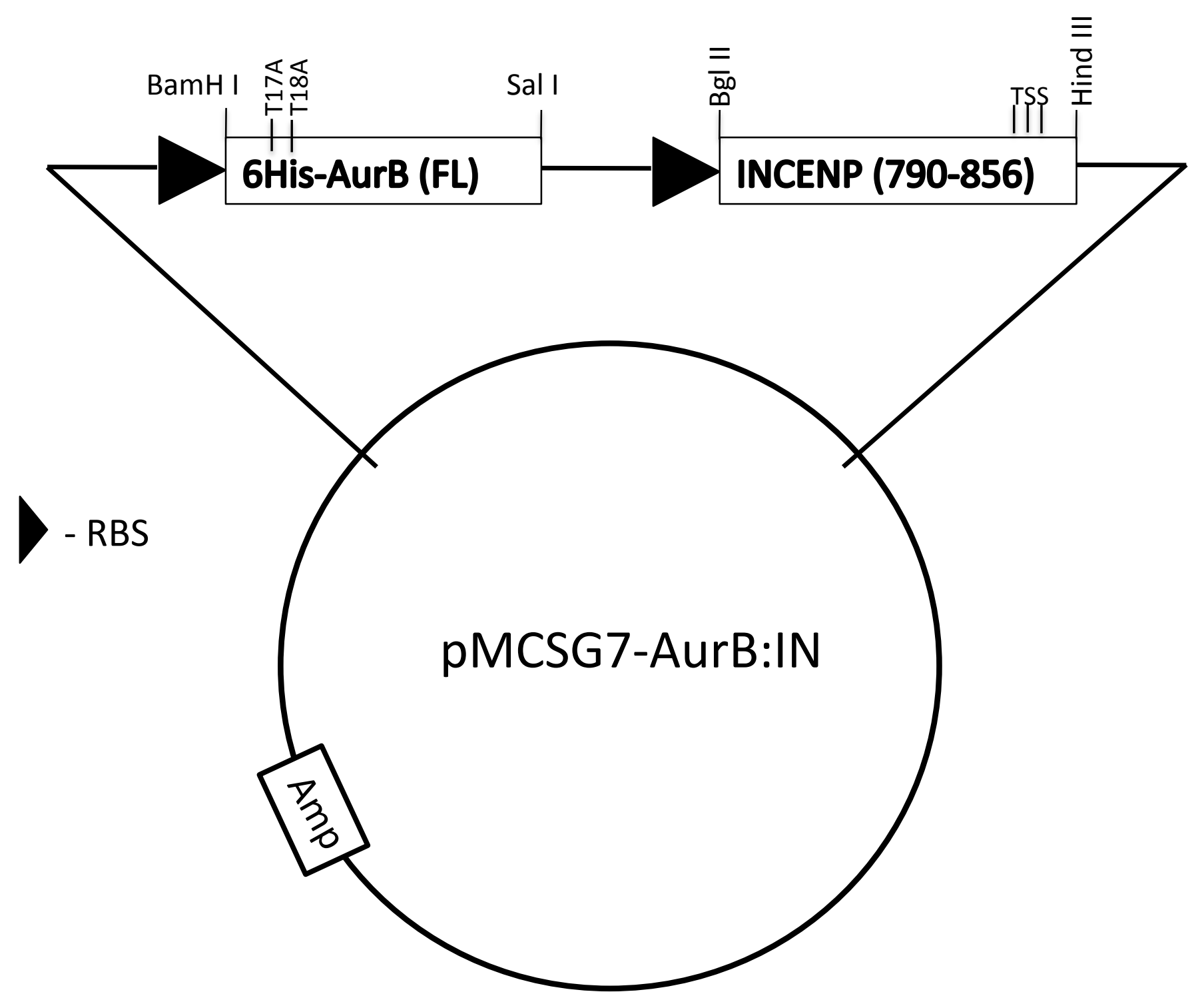


pMCSG7-6His-AuroraB (K122R):INCENP-IN-Box(790-856)

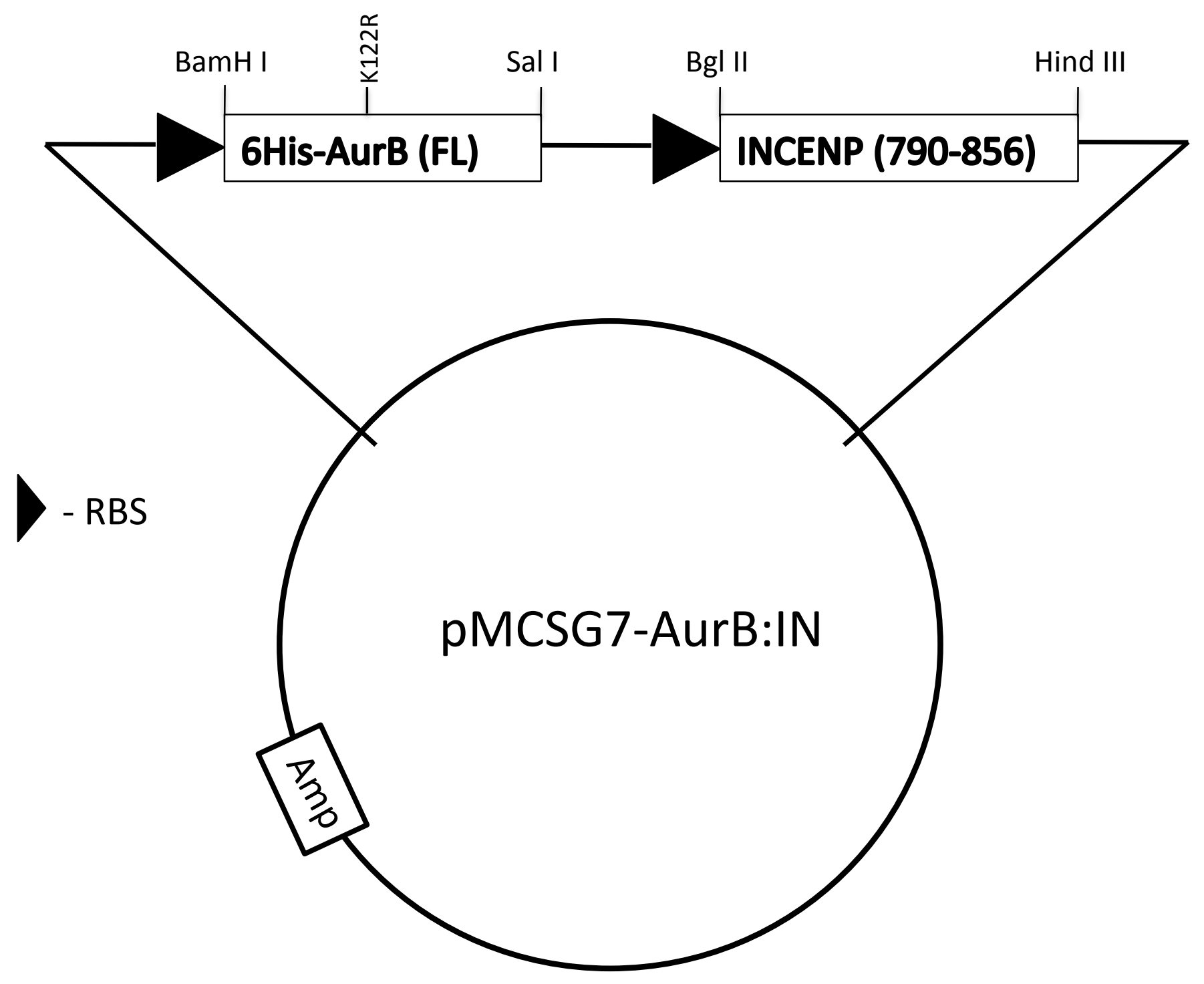


pMCSG7-6His-AuroraB (K122R):INCENP-IN-Box(790-847)

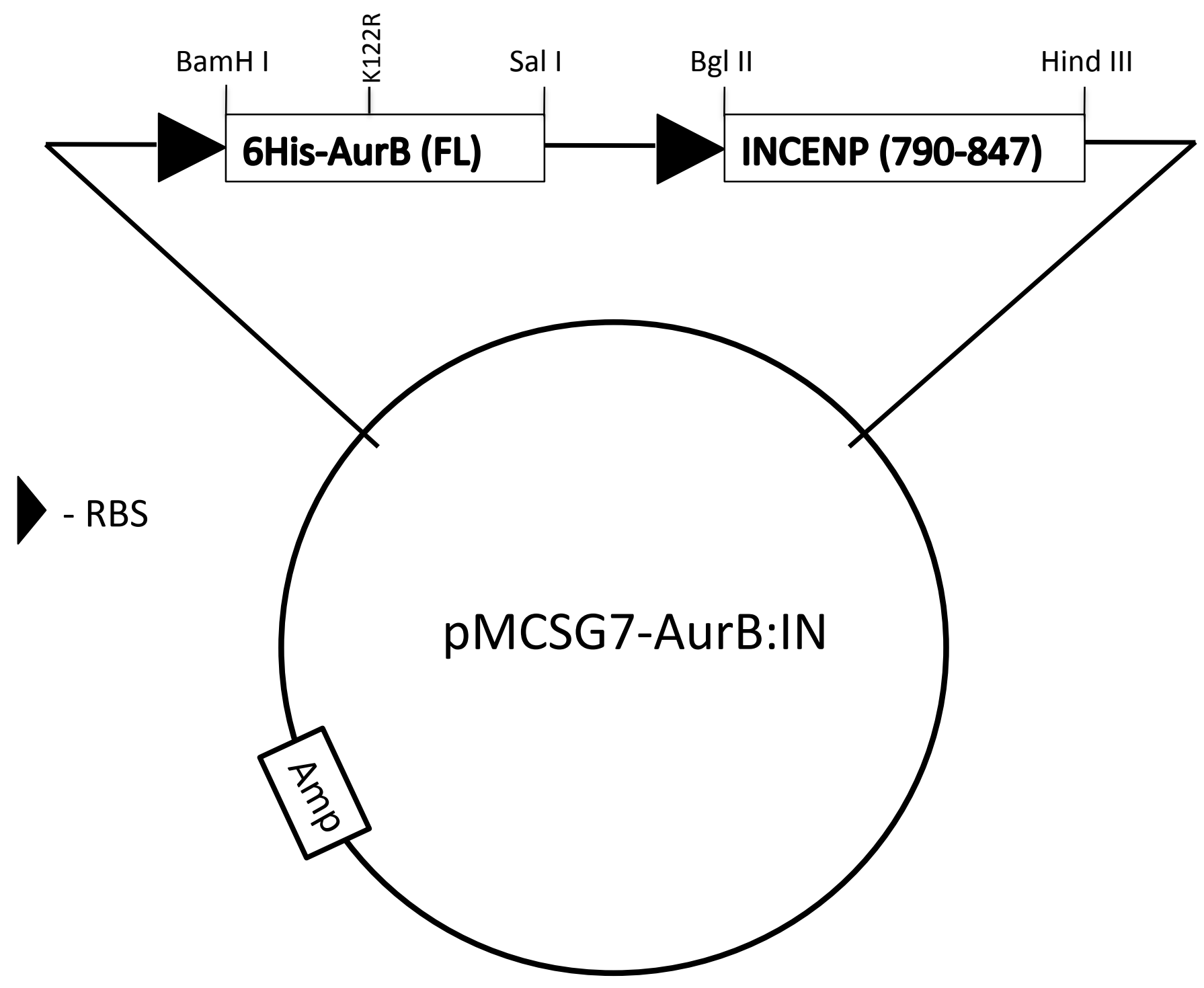


pCS2+-INCENP (T848A)-GFP

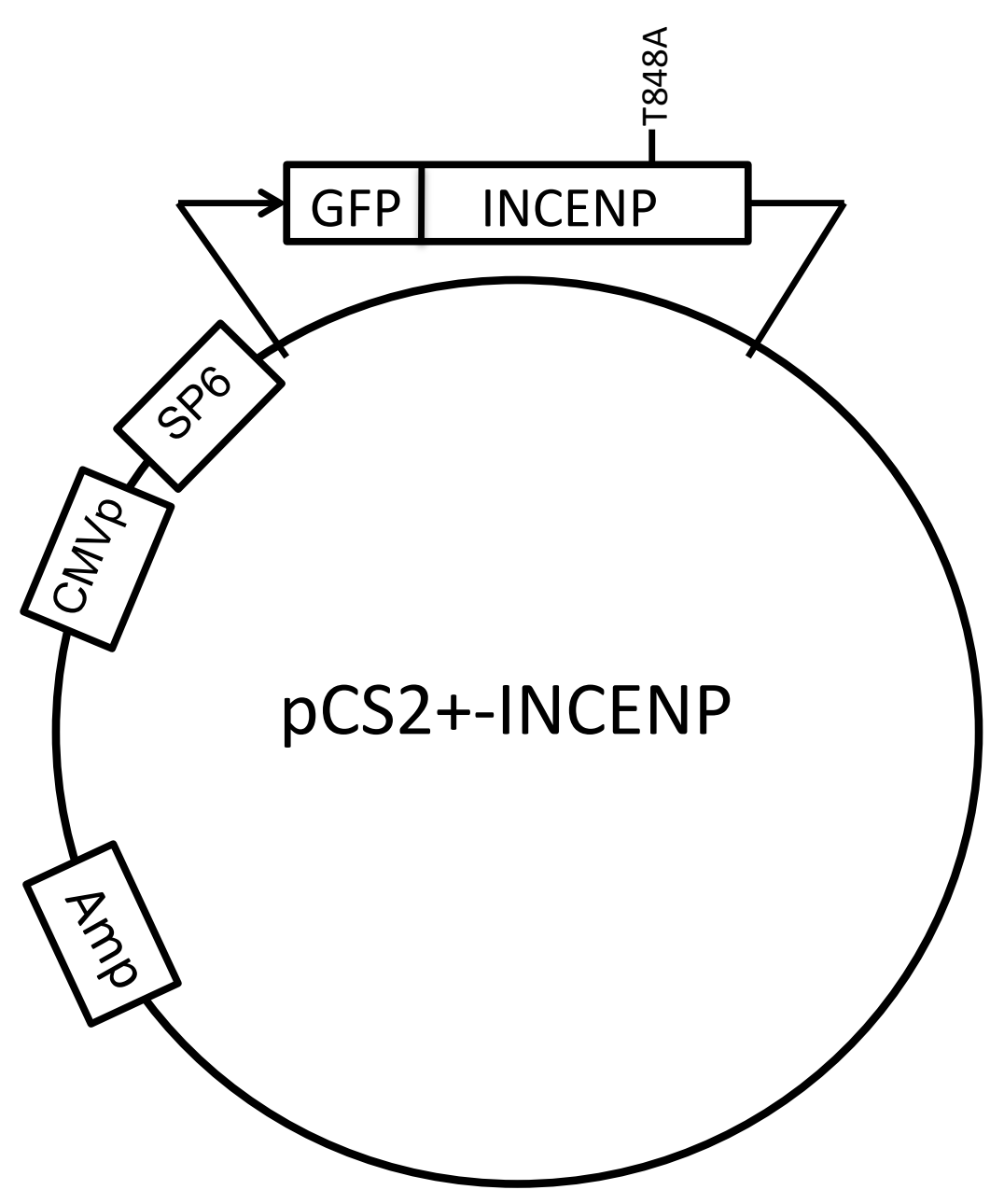

Site-directed mutagenesis performed on pCS2+(SP6):INCENP in Lab 
pCS2+-INCENP (S849A)-GFP

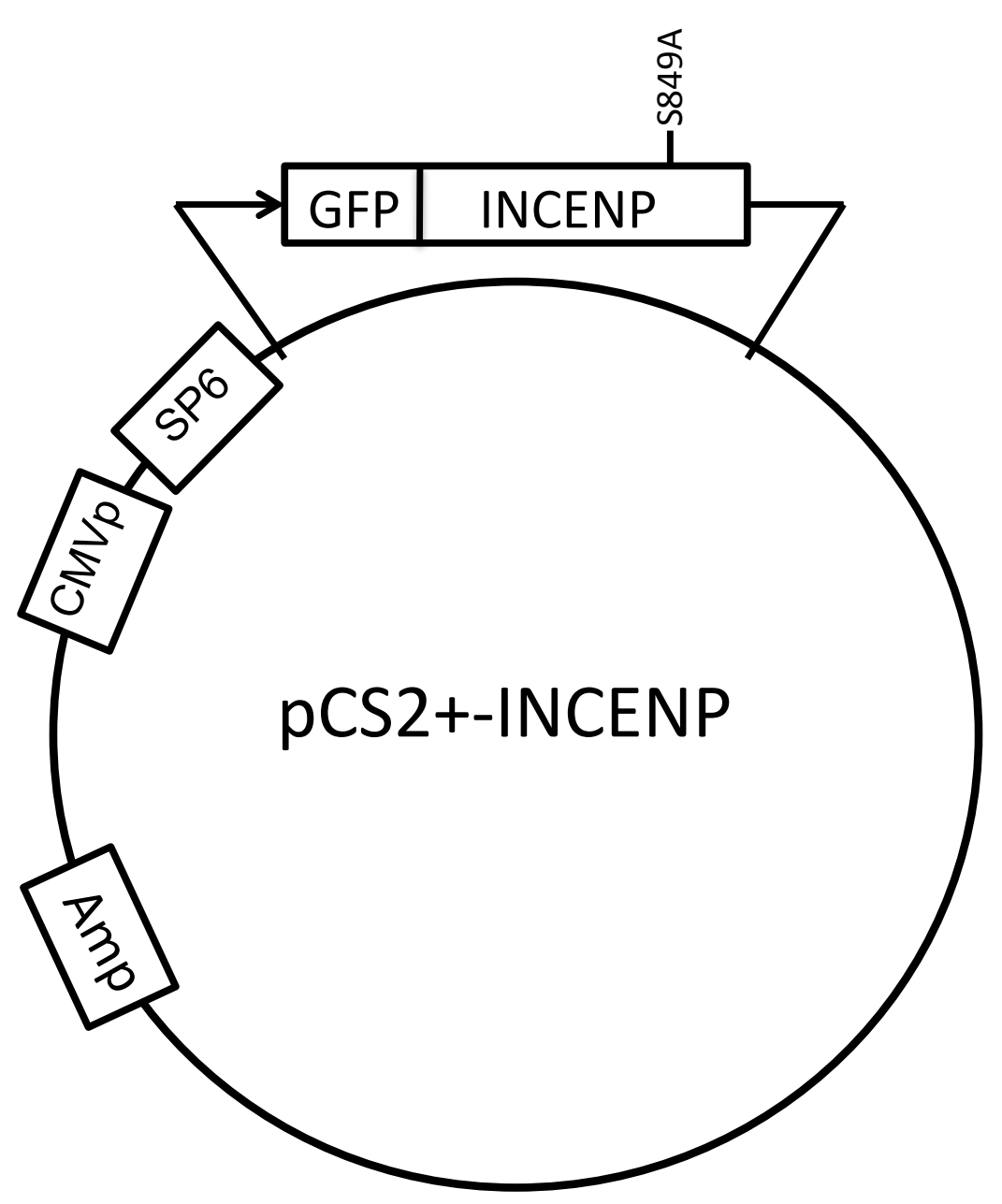

Site-directed mutagenesis performed on pCS2+(SP6):INCENP in Lab 
pCS2+-INCENP (S850A)-GFP

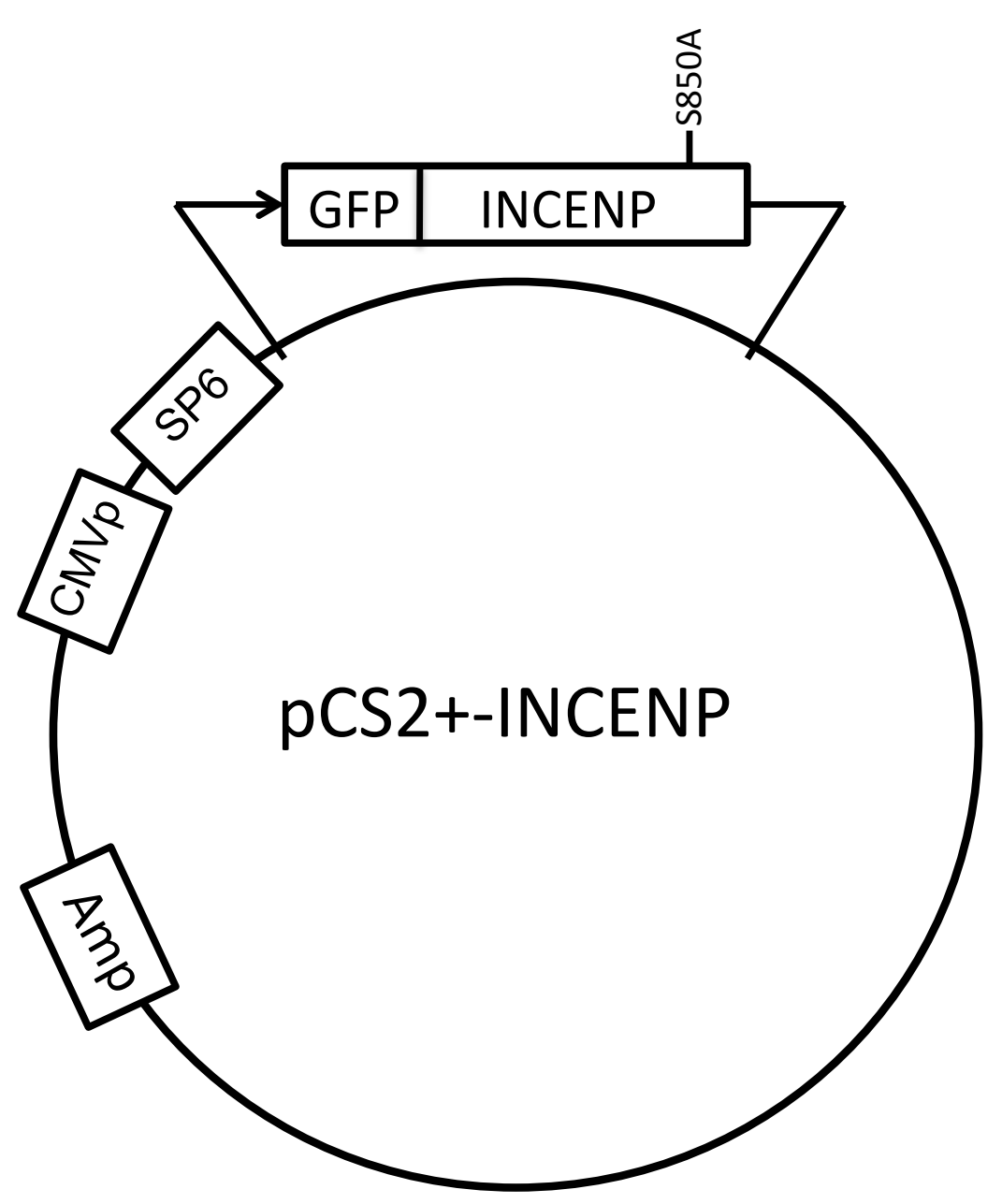

Site-directed mutagenesis performed on pCS2+(SP6):INCENP in Lab 


\section{pcDNA5.0/FRT-INCENP AAA(mutated TSS)-EGFP}

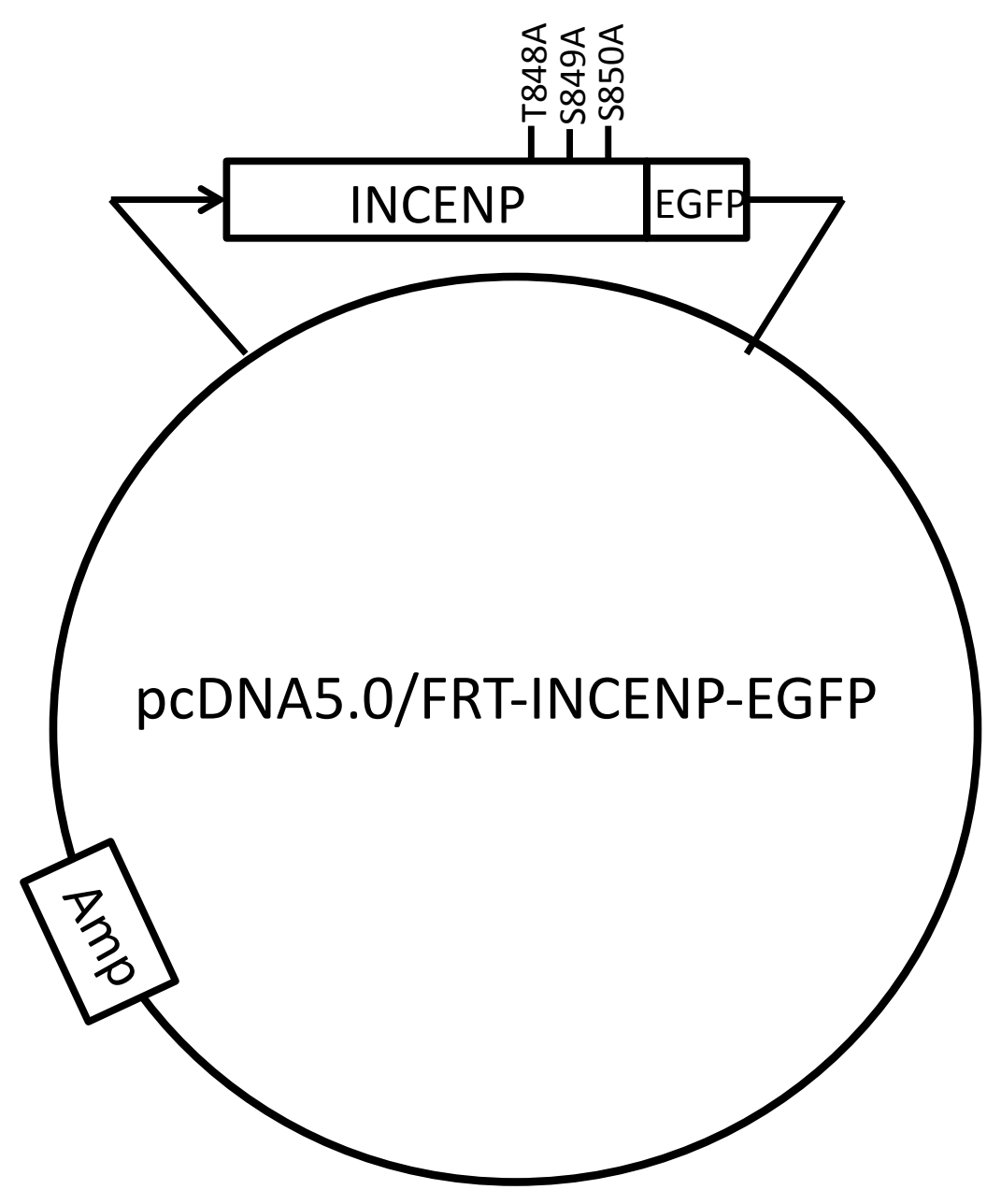

Site-directed mutagenesis performed on pcDNA5.0/FRT-:INCENP (made by Limin) in Lab 


\section{pCDNA5.0/FRT-INCENP EEE(mutated TSS)-EGFP}

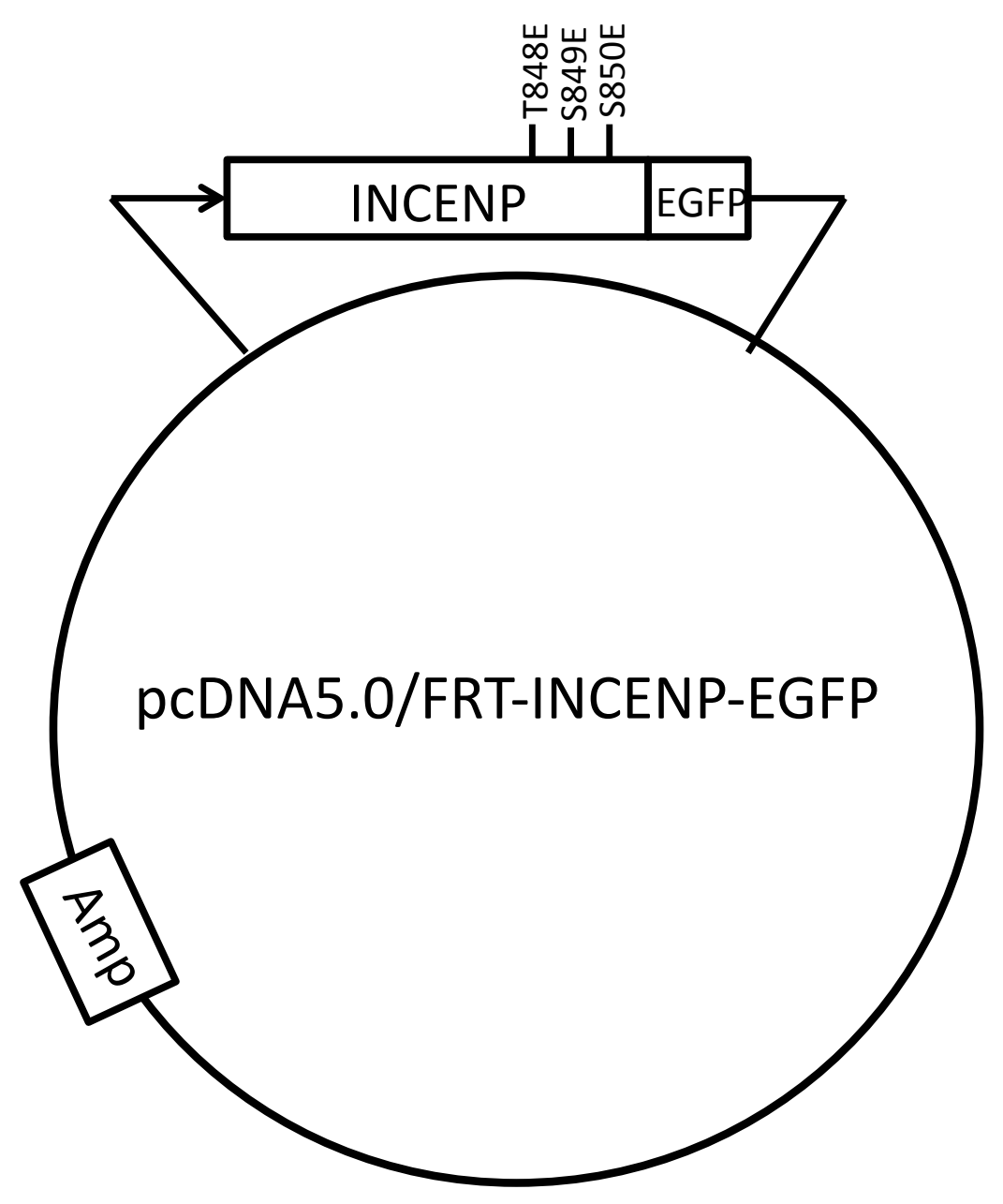

Site-directed mutagenesis performed on pcDNA5.0/FRT-:INCENP (made by Limin) in Lab 
pcDNA5.0/FRT-EB1-EGFP

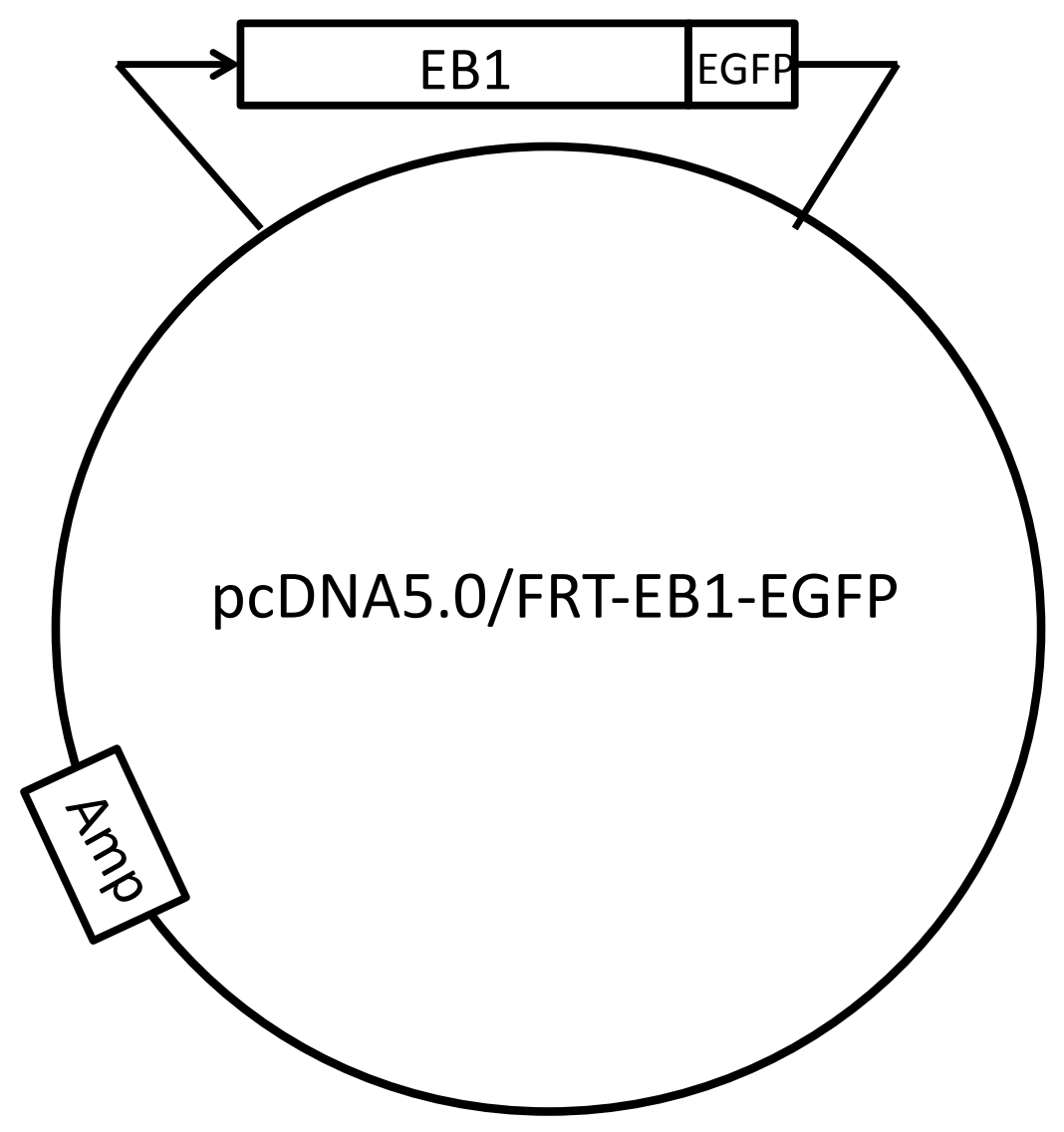


pcDNA5.0/FRT-EB1-siRes(CDS) -EGFP

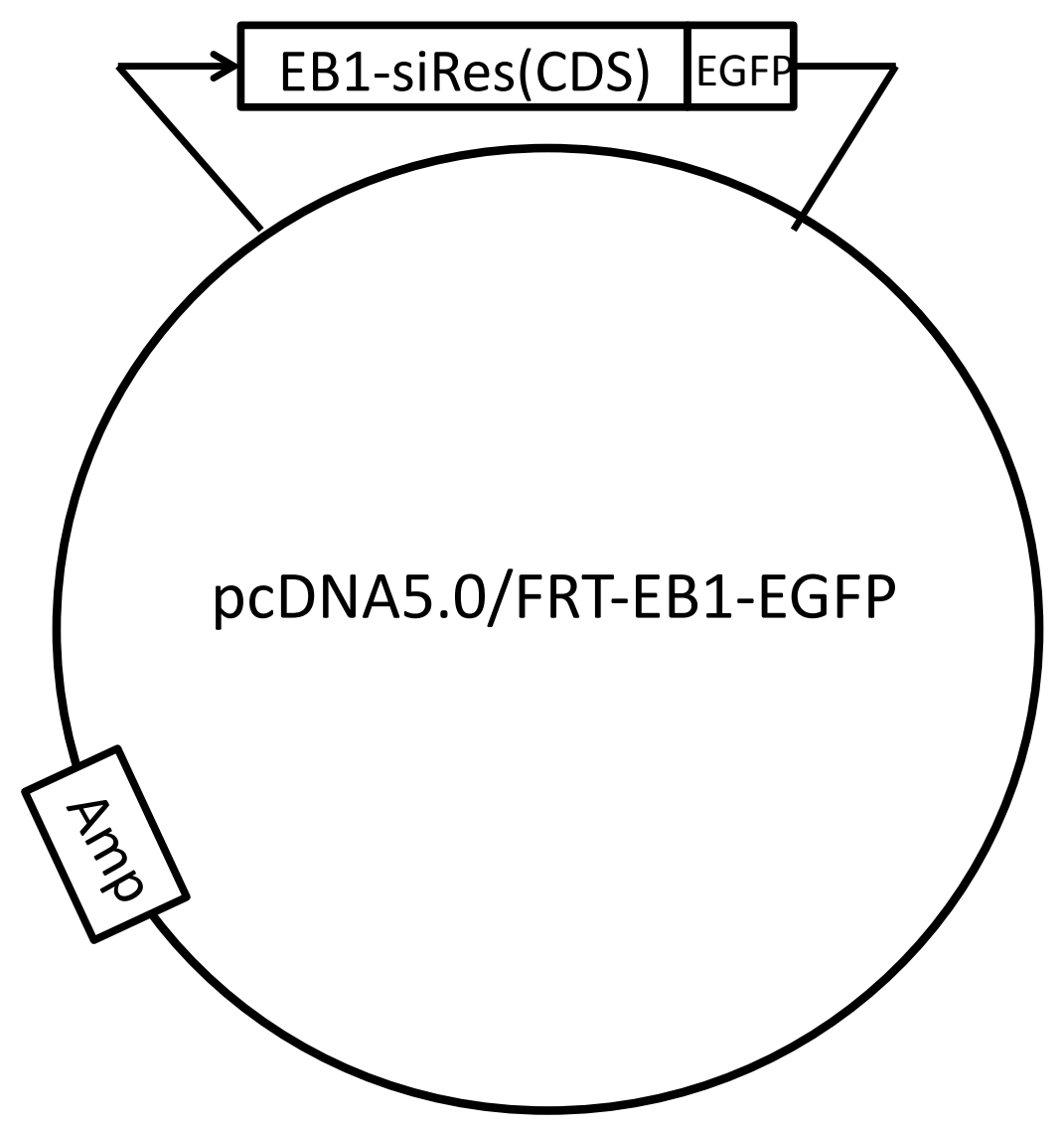




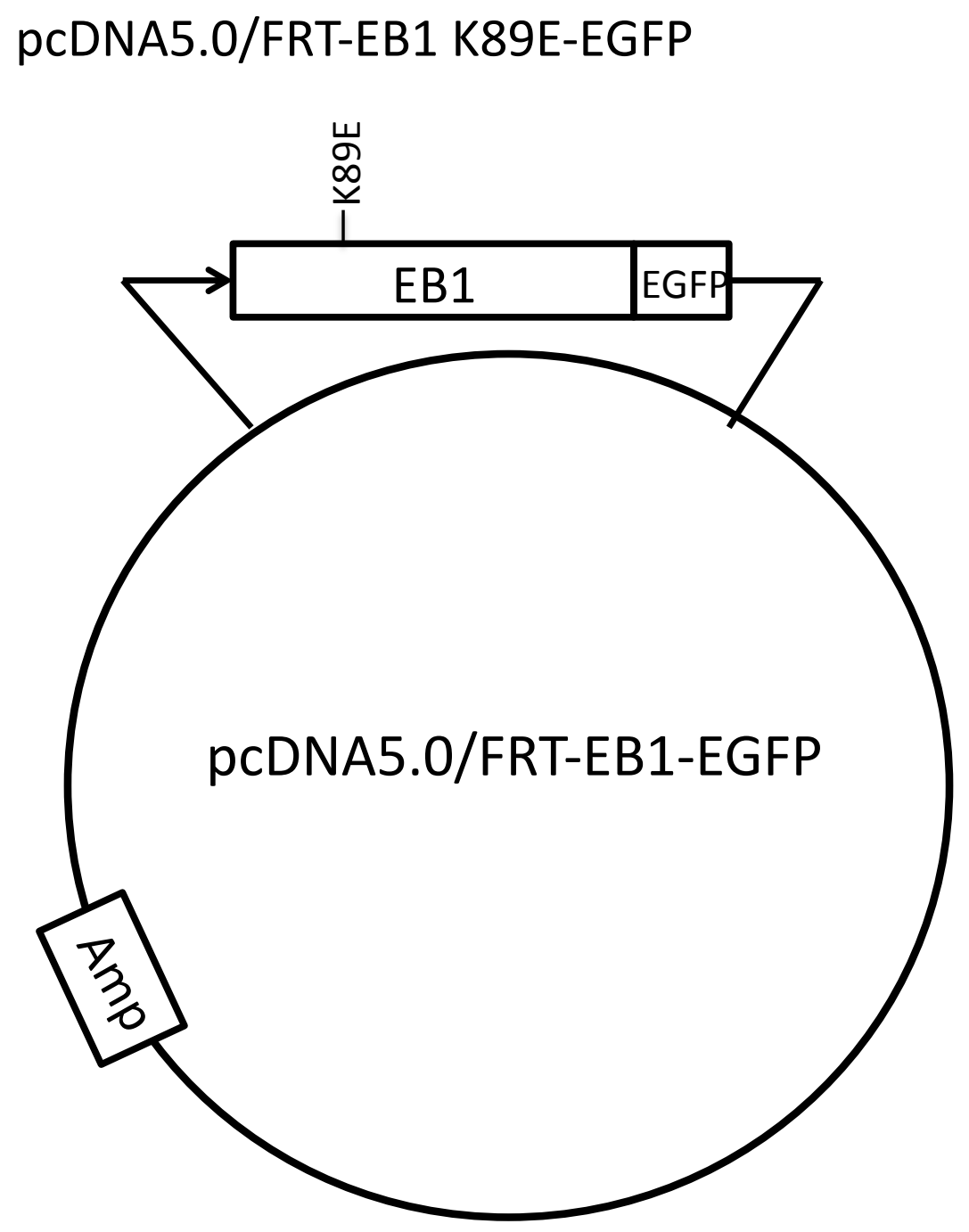


pcDNA5.0/FRT-EB1 siRes(CDS) K89E-EGFP

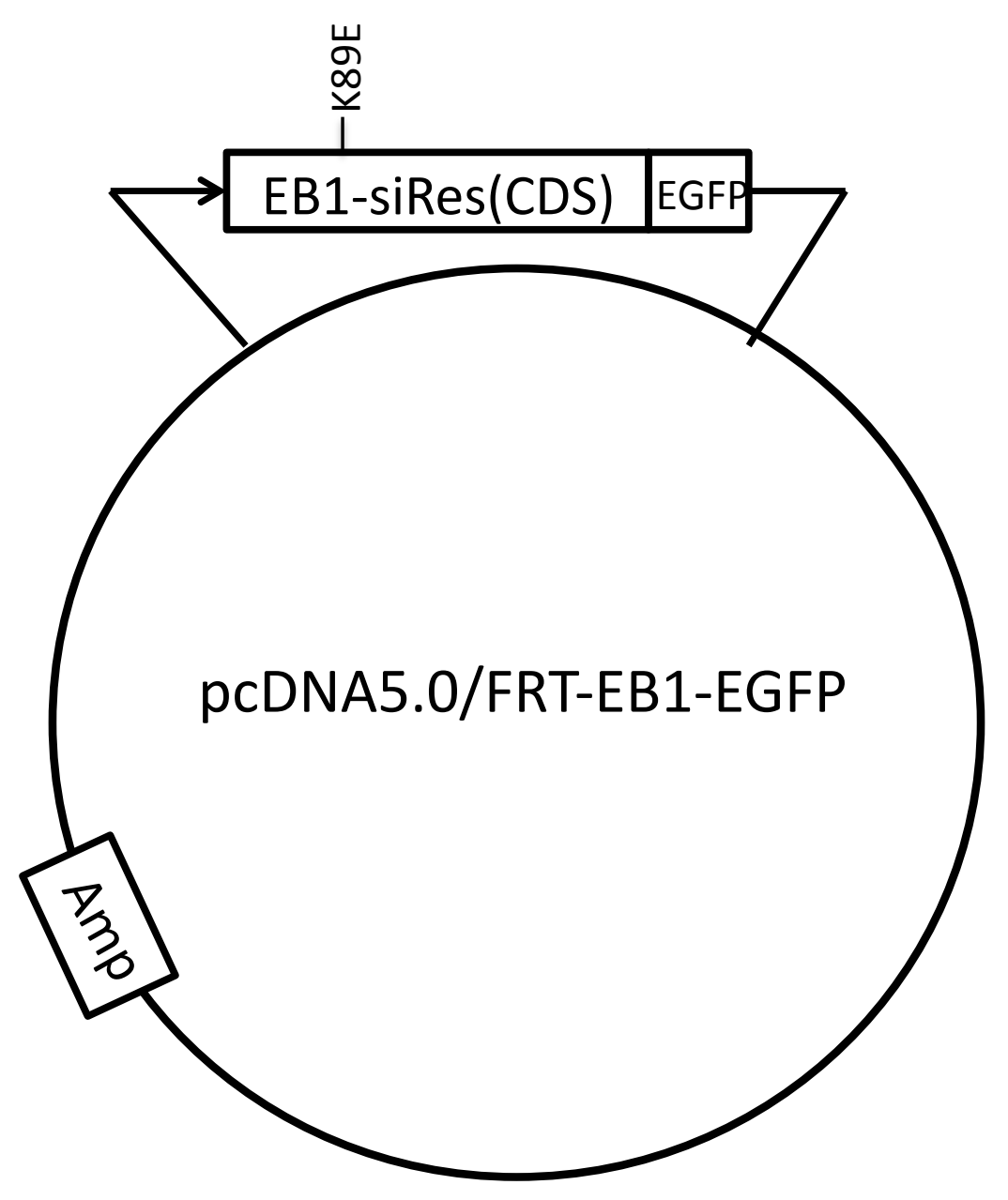

